\title{
Volatile organic compounds analysis in the context of gastrointestinal health and disease
}

Citation for published version (APA):

Baranska, A. L. (2021). Volatile organic compounds analysis in the context of gastrointestinal health and disease. [Doctoral Thesis, Maastricht University]. ProefschriftMaken.

https://doi.org/10.26481/dis.20211129ab

Document status and date:

Published: 01/01/2021

DOI:

10.26481/dis.20211129ab

Document Version:

Publisher's PDF, also known as Version of record

\section{Please check the document version of this publication:}

- A submitted manuscript is the version of the article upon submission and before peer-review. There can be important differences between the submitted version and the official published version of record.

People interested in the research are advised to contact the author for the final version of the publication, or visit the DOI to the publisher's website.

- The final author version and the galley proof are versions of the publication after peer review.

- The final published version features the final layout of the paper including the volume, issue and page numbers.

Link to publication

\footnotetext{
General rights rights.

- You may freely distribute the URL identifying the publication in the public portal. please follow below link for the End User Agreement:

www.umlib.nl/taverne-license

Take down policy

If you believe that this document breaches copyright please contact us at:

repository@maastrichtuniversity.nl

providing details and we will investigate your claim.
}

Copyright and moral rights for the publications made accessible in the public portal are retained by the authors and/or other copyright owners and it is a condition of accessing publications that users recognise and abide by the legal requirements associated with these

- Users may download and print one copy of any publication from the public portal for the purpose of private study or research.

- You may not further distribute the material or use it for any profit-making activity or commercial gain

If the publication is distributed under the terms of Article $25 \mathrm{fa}$ of the Dutch Copyright Act, indicated by the "Taverne" license above, 
Volatile Organic Compounds Analysis in the context of Gastrointestinal

Health and Disease 
Copyright Agnieszka Baranska, Maastricht 2021

Layout: Agnieszka Baranska

Cover: Krzysztof Baranski https://invisionsigns.co.uk/

Printed by: www.proefschriftmaken.nI

ISBN: 978-94-6423-558-6

The research performed in this thesis was conducted at NUTRIM School of Nutrition and Translational Research in Metabolism at Maastricht University, that participates in the Graduate School VLAG (Food Technology, Agrobiotechnology, Nutrition and Health Science), accredited by the Royal Netherlands Academy of Arts and Sciences. Part of the research present in this study has been funded by the Top Institute of Food and Nutrition (TIFN), Wageningen, as well as, Nutricia Research, the Netherlands. 


\title{
Volatile Organic Compounds Analysis in the context of Gastrointestinal Health and Disease
}

\author{
PROEFSCHRIFT \\ ter verkrijging van de graad van doctor aan de Universiteit Maastricht, \\ op gezag van de Rector Magnificus, Prof. Rianne Letschert \\ volgens het besluit van het College van Decanen, \\ in het openbaar te verdedigen \\ op maandag 29 november 2021 om 13.00 uur. \\ door
}

Agnieszka Lidia Baranska

geboren op 7 januari 1987

te Slupsk (Polen) 


\section{Promotor}

Prof. Dr. F.J. van Schooten

Prof. Dr. D. Jonkers

\section{Copromotor}

Dr. A. Smolinska

\section{Beoordelingscommissie}

Prof. Dr. E. Dompeling, voorzitter

Prof. Dr. E.C.M. Mariman

Dr. John Penders

Dr. Pierre-Hugues Stefanuto (University of Liège, Belgium)

Prof. Dr. Kristin Verbeke (KU Leuven, Belgium) 


\section{Table of contents}

Chapter 1: Thesis outline and introduction

Chapter 2: Factors that influence the volatile organic compound content in human breath

Chapter 3: Profile of volatile organic compounds in exhaled breath changes as a result of gluten free diet

Chapter 4: Comparing patterns of volatile organic compounds exhaled in breath after consumption of two infant formulae with a different lipid structure: a randomized trial

Chapter 5: Volatile Organic Compounds in breath as marker for Irritable Bowel Syndrome: a metabolomic approach

Chapter 6: Volatile Organic Compounds in exhaled air as novel marker for disease activity in Crohn's disease: A metabolomic approach

Chapter 7: VOCs dynamics in 'in vitro' set up: collection and analysis of volatile organic compounds from headspace of cell cultures

Chapter 8: Discussion

Summary and general discussion $\quad 170$

Study limitations $\quad 187$

VOCs in Gl Health 188

Future Perspectives 190

$\begin{array}{ll}\text { Impact Paragraph } & 199\end{array}$

$\begin{array}{ll}\text { Acknowledgements } & 207\end{array}$

$\begin{array}{ll}\text { Curriculum vitae } & 213\end{array}$

$\begin{array}{ll}\text { Portfolio } & 215\end{array}$ 



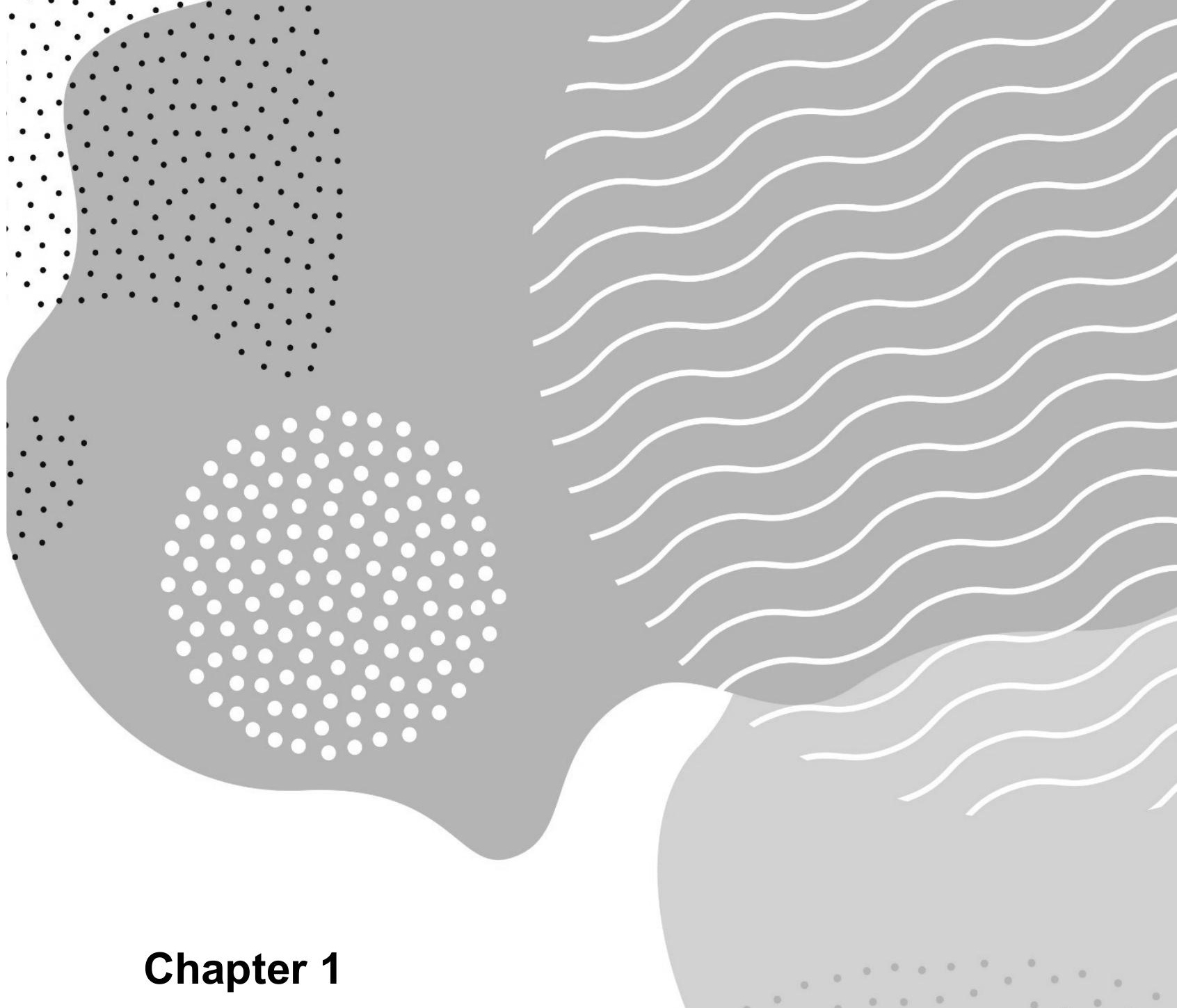

Thesis outline and introduction 


\section{Thesis outline}

The main aim of this thesis is to describe the potential of volatile organic compounds (VOCs) analysis to gain insight into different aspects of Gastrointestinal (GI) health and disease. A schematic overview of the contents and questions addressed in this work can be found below (Figure 1). Most of the work described in this thesis is devoted to the analysis of VOCs originating in the human body, by collecting and measuring breath samples from healthy subjects and from Irritable Bowel Syndrome (IBS) and Inflammatory Bowel Disease (IBD) patients. The introduction of this thesis will focus first on $\mathrm{Gl}$ Health and Disease and then on the general background on VOCs analysis. In Chapter 2, we systematically access influence of several endogenous and exogenous factors on exhaled breath composition. Next, we explore whether breath analysis can be used to track dietary changes (Chapter 3 ) and digestion patterns (Chapter 4) in healthy individuals. In Chapter 5, we investigate the diagnostic potential of breath analysis to be used in IBS recognition by studying samples coming from a well-defined IBS cohort in comparison to GI healthy individuals. Here, the relation of potential VOC biomarkers to GI symptoms reported in IBS subjects and in 1300 individuals from a general population is studied. In Chapter 6, we investigated whether VOCs can accurately differentiate between active $C D$ and in remission and become a non-invasive and reliable alternative for presently used diagnostic methods.

In order to gain insight in the in vivo production of VOCs by human cells and its influence on the exhaled breath composition, we show the development and validation of a system for dynamic collection of headspace air in vitro with the use of Caco-2 cell line as model for the intestinal epithelial barrier in Chapter 7.

Finally, in Chapter 8, the results presented in this thesis, their meaning and future perspectives are discussed. It also explains in more detail issues and challenges present in breath research. 


\section{Volatile Organic Compounds in the context of Gastrointestinal (GI) Health and Disease}

Chapter 1: Introduction on GI Health and Disease; Volatilome in Breath and Headspaces of Cell Cultures

Chapter 2: Influence of external factors on composition of exhaled Breath

Should age, gender, BMI, smoking and medication be considered as confounding factor in analysis?
GI Health
GI Disorder
CI Disease

Chapter 3: Influence of gluten free diet on exhaled breath composition in healthy individuals.

Chapter 4: Influence of dietary intervention on exhaled breath composition in young, healthy adults.

Can we track dietary changes by studying exhaled VOCs?
Chapter 5: Potential of VOCs being a marker for the presence of IBS

Can we discriminate IBS individuals from GI healthy subject by comparing exhaled breath profiles?

How these possible biomarkers correlate with symptoms in IBS cohort?

What is the screening potential of investigated biomarker to be used in general population in a earch of IBS susceptive cases?
Chapter 6: Potential of VOCs being a tool to accurately assess and differentiate between active $C D$ and remission

Can we replace currently functioning standards?

Chapter 7: Introduction and validation of the system for dynamic collection and analysis of volatile organic compounds from the headspace of cell cultures

What are the compounds released consumed by Caco-2 cell line, the model of intestinal barrier, in normal cell culture conditions?

IN VITRO

What are future implementations of findings?

IN CELL

CULTURES

HEADSPACES

Chapter 8: Discussion of results and challenges facing Breath Analysis 


\section{Introduction}

\section{Gastrointestinal Health and Disease}

The subject of gastrointestinal (GI) health and its importance can be traced back to different points in the human history. 'All diseases begin in the gut' is a famous quote thought to be nearly 2500 years old and one of the remaining wisdoms of Hippocrates. Long ago, Asian medicine recognized the importance of the abdomen believing it to be 'onaka' - the center of the soul and 'hara' - the center of spiritual and physical strengths [1]. It was also more than 100 years ago when for the first time the connection was made between mental health and our gut. Many $18^{\text {th }}$ and $19^{\text {th }}$ century scientist believed that autointoxication caused by accumulating waste in colon was related to conditions such as depression, anxiety and psychosis [2]. We all have heard or used phrases as 'having a gut feeling', 'follow your guts' or 'trust your gut instinct'. Today the modern advancement in medicine and technology clearly shows the evidence on the importance of our GI health, not only to GI health per se but to our overall health as well. Increasing knowledge on brain-gut-axis and the importance of entire enteric nervous system resulted in the term 'second brain'. Nowadays, it is generally acknowledged that GI health cannot be simply understood as a lack of $\mathrm{Gl}$ disease and that its role is much more than effective digestion and absorption of fluids and nutrients. In 2011, Bischoff had attempted to redefine the term 'gut health' that was repeatedly used in scientific literature and industrial environment [3]. Together with the scientific committee he proposed five major criteria that might form the basis for a positive definition of gut health.

These five criteria are:

(i) effective digestion and absorption of food meaning normal nutritional status and effective absorption of minerals, food and water, combined with normal bowel movement and no Gl complaints

(ii) absence of GI illness, such as colorectal and other GI cancers, inflammatory bowel disease (IBD), coeliac disease (CD), acid peptic disease, acid reflux etc., as well as no enzymes deficiencies or carbohydrate intolerances 
(iii) normal and stable intestinal microbiota, meaning absence of bacterial overgrowth, normal composition of microbiota and no Gl infections nor antibiotic associated diarrhea

(iv) effective immune status, meaning properly functional GI barrier with normal mucus production, normal levels of immune cells, and no allergies or hypersensitivities present

(v) status of well-being with normal quality of life and positive gut feeling, additionally, supported by proper serotonin levels and normal function of enteric nervous system.

The difficulty with those criteria is (in part) the subjectivity of what 'normal' means as it cannot always be defined by objective parameters. Nevertheless, the Bischoff work provides a comprehensive overview of factors worth considering when studying gut health. While several of these criteria are very much self-explanatory, two particularly interesting entities are considered key in gut homeostasis and should be mentioned in more details, namely GI microbiota and GI barrier.

\section{GI microbiota}

The GI track is the home of over $10^{14}$ microbes including bacteria, archaea and eukaryote, inhabiting and forming complex mutualistic symbiosis with our body [4]. Recently, it has been found that the human to microbial cell ratio is closer to 1:1 [5], than the previously reported 1:10 [4, 6]. A balanced and rich microbiota seems to play crucial role in our health and general wellbeing. The intestinal microbiota has several important functions for the host: i.e., providing colonization resistance, influencing the maturation, and functioning of the intestinal epithelium, barrier function and the immune system, and possesses a large metabolic capacity. By the latter, it results for example in the production of vitamins (e.g., B/K) and beneficial short chain fatty acids from carbohydrate fermentation [7].

When addressing the intestinal microbiota in relation to disease, it is important to first define a 'healthy' microbiota [8]. This remains however challenging given the large interindividual variation. Research did identify 'common core' - species that are 
present in all healthy subjects $[9,10]$. This includes well known species or genera such as: Bifidilobacterium, Eubacterium, Clostridium, Colinsella, Ruminococcus and Streptococcus, Akkermansia muciniphila. Currently, over 25 diseases or syndromes have been related to perturbations in intestinal microbiota perturbations, including e.g., IBD, IBS [11-13], colorectal cancer [14], obesity [15] and type 2 diabetes [16]. In IBD, both Crohn disease (CD) and ulcerative colitis (UC) are associated with reduction in diversity of the microbiota. Additionally, CD patients were repeatedly reported to have, among others, decreased levels of Faecalibacterium prausnitzii, a butyrate producer that is thought to have anti-inflammatory properties in a mouse mode [17], as well as increased levels of Enterobacteriaceae [18]. In another study, relative abundance levels of a mucus degrading, propionate producing bacterium, Akkermansia muciniphila, previously reported as one of the 'common core', were 10fold lowered in CD and 100-fold in UC when compared to healthy individuals [8]. In a mouse model, this bacterium had proven to have notably stimulating effects on the immune system and barrier function [19, 20]. In IBS, few studies reported the decreased levels of Dorea and Ruminococcus when compared to healthy individuals who showed increased levels of Bifidobacterium and Faecalibacterium prausnitzii, $[11-13,21]$. Lactobacillus was reported to be decreased in IBS patients by one research group [22] but was increaded in IBS-diarrhoea predominant type (IBS-D) as reported by another research group [23]. Altered microbiota was also found in diseases like Alzheimer disease [24], Parkinson's [25], and Rheumatoid arthritis [26]. Furthermore, around $70 \%$ of children with autistic spectrum disorder (ASD) seem to be affected by a form of $\mathrm{Gl}$ disorder and differences were found in microbial composition in a range of autistic spectrum disorders when compared to healthy individuals [27-29]. So far it is unclear if an altered microbiota composition is a cause or the effect of a disease, but growing evidence points to its direct or indirect relation to various underlaying mechanisms.

Many connections as such are present in the literature but, nevertheless much more research needs to be performed to validate these results and to learn more about real potential and consequences of gut microbiome perturbations in terms of pathophysiology, diagnosis, and treatment of GI disorders. 


\section{GI Barrier}

The Gl barrier consist of a single epithelial layer covered by e.g., mucus, antimicrobial peptides, and intestinal microbes and together with the underlying immune system forms an important barrier between luminal antigens, such as microorganisms and nutrients, and the interior of the body [30]. The immune system can discriminate between commensal microorganisms and pathogens, leading to immune tolerance and response to invaders, respectively. Antibacterial properties of gastric acid, bile and digestive enzymes contribute to the innate defence in the gut. The paracellular space is sealed by intercellular tight junctions, gap, and adherence junctions as well as desmosomes. Dysfunction of any of these can lead to impairment of GI barrier function and increased permeability [31] and is found in many diseases. In a previous study for example, it has been shown that ASD often coexists with GI complains and increased permeability also known as 'leaky gut' [32]. Animal models of ASD had confirmed that a 'leaky gut' allows the entry of bacterial products and toxins to the bloodstream, which can induce inflammation [33] and impact brain functions [34]. Several studies have also reported an increased permeability in IBD and IBS, especially in diarrhoea-predominant IBS [35], when compared to healthy controls [36, 37].

\section{Gut disorders and disease}

GI disorders, ranging from mild to severe disease are rather frequent and affect a large part of the population. The International Foundation of Gut Disorders (IFFGD) states that functional gastrointestinal disorders (FGID) and motility disorders are the most common cause of $\mathrm{Gl}$ disorders in the population. The true prevalence is difficult to establish as not all the people that feel gut discomfort seek medical attention. Nevertheless, FGID, are one of the most reported disorders worldwide [38, 39], with a prevalence up to $10-15 \%$. 'Functional' in this context relates to the presence of symptoms without clear organic cause. As no structural abnormalities can be seen by endoscopy, x-ray, or blood tests, diagnostics is based on symptoms and exclusion of other diseases when indicated $[38,40]$. In this thesis we focus on IBS as a form of FGID. 


\section{Irritable Bowel Syndrome}

IBS is a heterogeneous disorder with a multifactorial, though not completely understood pathophysiology, including alterations in e.g., intestinal motility, visceral perception, intestinal microbiota, barrier function, low-grade immune activation, and psychosocial factors [41]. The first criteria defined to aid the diagnosis of IBS were made by Manning in 1978 [42]. Later, in 1989, The Rome Foundation developed and published new criteria for IBS, updated since several times. While the studies in this thesis were performed it was Rome III criteria, published in 2006 that were considered. The IBS clinically diagnosed subjects from the Maastricht IBS cohort were identified in accordance with these criteria. Since then, IV revision was completed and released in 2016 [39, 40]. The changes between III and IV criteria with regards to IBS [43] will be shortly discussed in the last Chapter 8 of this thesis.

Nowadays diagnosis of IBS is based on recurrent abdominal pain at least 1 day per week, which is associated with changes in stool consistency and frequency and pain release before, during or soon after defecation. The symptoms onset must be present for at least 6 months. In addition, organic diseases that can mimic IBS-like symptoms such as IBD, thyroid dysfunction, enteric infections, celiac disease, and colorectal cancer must be excluded. Because the prevalence of organic diseases in potential IBS is low and diagnostics tests are often invasive and costly, these exclusions are only performed when typical IBS symptoms appear in the presence of red flags such as: rectal bleeding, iron deficiency, weight loss and family history of organic diseases mentioned earlier.

Among the general population in Western countries, IBS has a prevalence of up to $15 \%$ based on Rome III criteria [44-46] with a clear female predominance. Soares states in her review that IBS can affect even up to one in five people at some point in their life [41]. IBS causes a major burden in quality of life and work productivity of patients, but also in health care costs [47]. A recently published study reported that on average IBS patient misses 2 days of work per month and his productivity suffers on average 9 days per month [48]. 
Heterogeneity of IBS is described in the several subtypes based on predominant bowel habits i.e., diarrhoea (IBS-D), constipation (IBD-C), a mixed type (IBS-M) and undefined one (IBS-U). Even though subtypes can aid adjusting therapeutic treatment [49] it has been reported for large number of patients that they change over time [50]. Furthermore, most pathophysiological factors were not found to be associated with specific subtypes and treatment efficacy is often limited. Therefore, it is important to further unravel factors underlying predominant symptoms in IBS.

In the past decade there was considerable progress in identifying factors associated with IBS, such as, diet, antibiotic use, prior infections, and psychological state [51]. About $70 \%$ of patients consider their symptoms to be food related. Psychological state and psychiatric comorbidities, especially depression and anxiety, are commonly present among IBS patients (i.e., up to $60 \%$ ) [52]. These and other factors can potentially influence the $\mathrm{GI}$ barrier, immune function and the intestinal microbiota composition and activity [53]. Furthermore, genetic and epigenetic predisposition to IBS is also reported, but that research is still in its infancy stage [54].

In recent years, several treatment options were proposed for easing IBS symptom such as lubiprostone and linaclotide for IBS-C or rifaximin and eluxadoline for IBS$\mathrm{D}$, but merely only benefit small number of patients. Moreover, dietary changes may be helpful, especially in patients who related their symptoms to ingestion of different foods, mainly carbohydrates and fat $[55,56]$. A few studies have shown that fermentable oligosaccharides, disaccharides, monosaccharides, and polyols (FODMAP) may cause bloating and a low FODMAP diet leads to a significant overall symptom improvement in about $70 \%$ of IBS patients $[57,58]$. In the long term, low FODMAP diet is associated with an increased risk of developing nutritional deficiencies, especially due to a low fiber intake, and the decrease of beneficial bacteria and metabolites [59]. The low FODMAP diet should be conducted under supervision or education led by experienced dietitian and should include reintroduction of an acceptable dose of fermentable food compounds. It is worth noting that a general dietary advice for IBS patients as defined by British National Institute for Health and Care Excellence, i.e., NICE diet, could be first in line to try as 
there were no significant differences noticed when both diets were compared [60]. This is also in line with the advice from British Dietetic Association in 2016 [61].

The search for biomarkers in IBS is difficult as no concrete organic cause can be determined for this functional disorder. There is, however, more and more evidence pointing to subtle organic changes in different biological processes that may be involved in IBS aetiology. Markers assessing intestinal permeability, visceral hypersensitivity, genetic markers, and markers of inflammations are only some of the examples studied while searching for an IBS biomarker [62] but thus far these do not apply to all patients. Also, a combination of several serum and genetic markers was considered [63]. Unfortunately, so far, neither single or panels of potential biomarkers has been accepted for use in clinic and general practice. In Chapter 5, we use a holistic, -omics approach to find subtle differences in overall volatile profile of individuals with IBS as compared to healthy GI status, and to relate these to symptom patterns.

\section{Inflammatory Bowel Disease}

Crohn's disease (CD) and ulcerative colitis (UC) are both chronic relapsing inflammatory diseases of the gastrointestinal tract - collectively referred to as IBD [64]. IBD is generally considered to be result of dysregulated mucosal immune response to gut microbes in genetically susceptible individuals [65]. In their review, Burirsh et al., have shown that $0.3 \%$ of European population suffers from IBD with the highest rates reported in Scandinavia and UK. Recent data show that the incidence of IBD is increasing [66, 67]. IBD is a lifelong disease and is mainly diagnosed in young adulthood. Although it is not exactly clear what triggers the onset of IBD, research of Tsianos et al. found associations with smoking, antibiotic use and oral contraceptives and/or diet [68]. IBD severely impacts on quality of life (QoL) and patients report higher levels of psychiatric distress and anxiety compared to people without other chronic disease [69]. Furthermore, IBD is also associated with high direct and indirect health care costs.

In this thesis, in Chapter 6 we focus on CD. While UC is affecting the rectum and colon and is limited to inflammation of the epithelial lining of the gut, CD is a 
transmural inflammatory disease meaning it can affect the full thickness of the gut wall and additionally any part of the $\mathrm{Gl}$ tract [70]. In CD, chronic mucosal inflammation can lead to irreversible bowel damage with complications like stenosis or fistula $[71,72]$. Assessing disease activity is of major importance in improving long-term outcome and preventing complications [73]. At present, ileocolonoscopy is the gold standard for assessment of active disease, but this is an expensive and invasive procedure. In many studies, clinical activity indices have been used, such as the Crohn's Disease Activity Index (CDAl). However, this is not a reliable tool for prediction of endoscopic disease activity, as has been shown by Requeiro et al., in 2011 [74]. Two less invasive biochemical markers are available in clinic today: Creactive protein (CRP) and Fecal Calprotectin (FCP). The first one lacks specificity, as CRP is a general marker for inflammation, not necessarily linked to the gut. FCP, indicative for migration of neutrophiles to the intestinal mucosa, does reflect intestinal inflammation but indefinite findings occur in about a quarter of the patients [75]. In Chapter 6 we present a study that investigates whether VOCs in exhaled breath can accurately differentiate between active $C D$ and remission.

\section{The 'Omics' Era}

Both IBD and IBS would benefit from non-invasive markers for disease diagnosis and monitoring. Given the complex multifactorial etiology, combinations of markers reflecting various domains should be considered via a holistic approach. 'Omics' technologies allow for a holistic view and a comprehensive analysis of molecules that originate from various cells, tissues or the organism as a whole. Not long ago, scientists were accustomed to hypothesis-driven 'bottom up' research, where the question comes first, followed by examination of interactions between genes, proteins, and metabolites in individual cell types [76]. Nowadays a 'top-down' approach gained popularity, where scientists look for changes on different biological levels in a system medicine approach, to capture complexity of human body and find leads to new hypothesis [77].

The oldest of all omics, genomics, refers to universal collection of genes. It allows for identification of genetic variations associated with disease. Reversible 
modification of DNA and DNA associated proteins are studied by epigenetics. Transcriptomics focuses on RNA, molecular intermediate between DNA and proteins, while proteomics studies peptides abundances, modification, and interactions [77]. An emerging and relatively new field of 'microbiomics' studies all microorganisms of specific community together (e.g., human skin, gut) and their functional potential. Metabolomics, the omics of the biggest interest in this thesis, allows for comprehensive analysis of all low-molecular weight $(<1 \mathrm{kDa})$ small molecules/metabolites produced or present in a biosystem (i.e., cell, biofluid, tissue or organism collectively. Relevant definitions as provided by Goldsmith et al., are given in Table 1 [78].

\begin{tabular}{ll}
\hline Term & Definition \\
\hline Metabolite & $\begin{array}{l}\text { Small molecules that participate in general metabolic reactions and that are } \\
\text { required for the maintenance, growth, and normal function of a cell }\end{array}$ \\
\hline Metabolome & The complete set of metabolites in an organism \\
\hline Metabolomics & Identification and quantification of all metabolites in a biologic system \\
\hline
\end{tabular}

Table 1. Definitions Related to Metabolomics by Goldsmith et.al., [78].

In contrast to other omics, metabolomics provides a link between genotype and phenotype [79], as metabolomics are often the functional endpoints [80]. The composition of the metabolome is affected by genome and proteome, as well as, environmental and lifestyle factors, medication use, and underlying disease. Technical advancements of 1960, when first mathematical methods were introduced for modelling metabolism in cells, as well as of 1980, technical developments of nuclear magnetic resonance (NMR), can be thought of as a beginning of metabolomics era [81]. VOCs are often collectively called volatilome and it is a special group of metabolites discussed in more detail below. 


\section{Volatile organic compounds}

VOCs are low molecular weight, carbon containing molecules that are generally characterized by high vapor pressure in room temperature and low solubility in aqueous fluid. Thousands of various volatiles belonging to for example alcohols, aldehydes, aromatic hydrocarbons, acids, are present in nature and have often an important signaling function. For instance, they are produced by plants to provide a defense against pathogens and by insects acting as pheromones or as cues for finding prey $[82,83]$. Isoprene for example is thought to be produced by plants as a response to heat and oxidative stress [84].

In humans, VOCs can be excreted in various biofluids such as urine [85], blood [86], saliva [86] or fecal samples [87]. There are two main approaches to study volatile metabolites present in or released in biofluids. First, direct sampling of the air surrounding the sample (blood, urine, saliva etc.,), called headspaces [85, 86, 88]. Secondly, solvent extraction of the volatiles from cells or tissues [89]. The latter approach involves purification steps, namely removal of non-volatile compounds and as such becomes more complicated and time consuming.

\section{VOCs in exhaled breath}

Breath is a source of exhaled volatiles and is a therefore a highly interesting noninvasive matrix in humans [90] to measure VOCs, and to gain insight into metabolic changes due to diseases, diet or other factors. Next to inorganic gases (e.g., $\mathrm{N}_{2}, \mathrm{O}_{2}, \mathrm{NO}, \mathrm{CO}_{2}$, and $\mathrm{CO}$ ) and non-volatile substances (e.g., isoprostanes, cytokines) a single breath sample contains hundreds of VOCs occurring in nmol//pmol/l concentrations. Mass Spectrometry based techniques allow the detection of hundreds of VOCs per individual and due to intra- and inter-individual differences, the total number of VOCs in a series of samples may reach thousands. 'Breathomics' focuses on health-related VOCs present is one's breath [91, 92]. The first established links between fragrant breath and state of health date back to the times of Hippocrates and ancient Greece, around 400 years BC: urine like smell (amines) was recognized as a failing kidneys symptom, whereas rotten apple smell (acetone) was a sign of uncontrolled diabetes. 
Many VOCs in exhaled breath are comparable between individuals and as such they are discarded as non-informative in a search for biomarkers. The added value lies in endogenously produced VOCs from specific perturbed pathophysiological processes. For example, VOCs from inflamed tissue and altered metabolic pathways enter the blood stream and are subsequently excreted via the lungs due to their low solubility. In addition, exhaled VOCs will be affected by the host microbiota either through influencing host metabolism or by producing VOCs themselves depending on the microbiota composition and the availability of substrates. Differences in exhaled VOCs composition due to pathophysiological and metabolic causes may be indicative for certain states of health or disease. For example, disproportionally high levels of acetone due to ketoacidosis can be indicative of diabetes [93, 94]. Isoprene, another highly abundant endogenous VOCs in breath, is a by-product of mevalonate pathway of cholesterol biosynthesis $[95,96]$ and show positive correlation with blood cholesterol levels [95]. Another source of endogenous VOCs production results from the imbalance between reactive oxygen species and antioxidants that leads to oxidation and degradation of for example cell membrane, resulting in changes in hydrocarbon levels [97, 98]. Ethane and pentane among others, are often hypothesised to be a marker of marker for oxidative stress. Nevertheless, the exact mechanism and dynamics for hydrocarbon production is not fully understood and findings are inconsistent. To get more insight in the origin and potential relation between oxidative stress and its impact on volatile compounds, Chapter 7 shows the development and validation of a cell culture system to study such processes in vitro.

As volatiles are widely present all around us, it is important to note that breath content can be influenced by different factors including pollution and behavioural habits such as diet, smoking, drinking or medication use. For reliable analysis it is crucial to acknowledge possible confounders in the search of biologically relevant markers. In Chapter 2, we aimed to investigate how age, gender, BMI and other personal characteristic influences the profile of exhaled breath. In Chapter 3 and Chapter 4, the relation to gluten free diet and ingestion of different milk formula, respectively, was studied. 


\section{Methodology and analytical approach}

A variety of methodological and analytical approaches, including sample collection, measurement and analysis are being applied in breath research and these depend on the research questions. Advantages and limitations of different approaches are discussed in Chapter 8 of this thesis and here only a short introduction to our approach is given.

The methodology implemented in all studies within this thesis was based on the collection of total mixed air into $5 \mathrm{~L}$ polymer Tedlar Bags. Following collection, a vacuum pump was used to pre-concentrate the sample and pull the content of the bag into stainless-steel two-bed sorption tubes, filled with carbograph 1TD/Carbopack X (Markes International, Llantrisant, UK). Thereafter, tubes were stored at room temperature until further analysis via Gas Chromatograph-Mass Spectrometer with time-of-flight detector type (GC-tof-MS). Detailed parameters of the method are mentioned throughout the chapters of this thesis and have been described more extensively by Van Berkel et al., [99]. Before GC-MS data could be analyzed, it must have undergone several pre-processing steps. Our preprocessing pipeline includes denoising, baseline correction, alignment across all samples, peak picking, combining same compounds peaks, as well as, converting peak areas into data matrix and this is described in detail by Smolinska et.al., [91]. Variety of machine learning tools were used throughout this thesis to analyze the data. Starting with an unsupervised exploratory step, including Principal Component Analysis (PCA) for visualization purposes and to detect certain outliers, spot potential groupings and trends in data points, followed by application of supervised tools. Here, a priori knowledge such as sample label, treatment groups and other subject characteristics were considered in order to explore existence of correlations in the context of the studied objective. In this thesis, Multivariate Analysis of Variance (rMANOVA) in Chapter 2 and Chapter 4, Analysis of Variance-PCA (ANOVA-PCA) in Chapter 3, Random Forest (RF) in Chapter 5 and Chapter 6. 


\section{VOCs in headspaces}

To gain insight in the in vivo production of VOCs by human cells, impacting exhaled breath composition, in vitro experiments involving relevant cells can be performed. Studying VOCs from headspaces of microorganisms and cell cultures can help us understand which VOCs are produced in 'normal' conditions and how they differ in a diseased/stressed environment. Chapter 7 introduces the development and validation of the system for continuous collection of headspaces air. The use of airliquid interface and Caco-2 cell line allowed mimicking model of intestinal barrier and testing which VOCs are released or consumed by cells when compared to cells exposed to hydrogen peroxide. The system allows the application of different cell lines, as well as different experimental setups including varying exposure times and treatment options while preserving cell viability.

\section{Summary}

As outlined earlier the aim of this thesis is to examine whether VOCs can be indicative of gut disorders. For this, I performed a population study using healthy persons to identify personal characteristics that influence exhaled VOCs patterns, dietary intervention studies in humans to examine the relation between diet and exhaled VOCs, clinical studies with IBS and IBD patients to discover diagnostic VOCs biomarkers, and an in vitro cellular study to shed light on the relation between oxidative stress and VOCs release. Finally, I put the results in perspectives and identify the hurdles to move the breath field further with the purpose to implement VOCs profiling in the clinical setting. 


\section{References}

1. Yu, F., et al., Traditional Chinese medicine and Kampo: a review from the distant past for the future. J Int Med Res, 2006. 34(3): p. 231-9.

2. Schmidt, C., Mental health: thinking from the gut. Nature, 2015. 518(7540): p. S12-5.

3. Bischoff, S.C., 'Gut health': a new objective in medicine? BMC Med, 2011. 9: p. 24.

4. Backhed, F., et al., Host-bacterial mutualism in the human intestine. Science, 2005. 307(5717): p. 1915-20.

5. Sender, R., S. Fuchs, and R. Milo, Revised Estimates for the Number of Human and Bacteria Cells in the Body. PLoS Biol, 2016. 14(8): p. e1002533.

6. Gill, S.R., et al., Metagenomic analysis of the human distal gut microbiome. Science, 2006. 312(5778): p. 1355-9.

7. Yoshii, K., et al., Metabolism of Dietary and Microbial Vitamin B Family in the Regulation of Host Immunity. Frontiers in Nutrition, 2019. 6.

8. de Vos, W.M. and E.A. de Vos, Role of the intestinal microbiome in health and disease: from correlation to causation. Nutr Rev, 2012. 70 Suppl 1: p. S45-56.

9. Salonen, A., et al., The adult intestinal core microbiota is determined by analysis depth and health status. Clin Microbiol Infect, 2012. 18 Suppl 4: p. 16-20.

10. Jalanka-Tuovinen, J., et al., Intestinal microbiota in healthy adults: temporal analysis reveals individual and common core and relation to intestinal symptoms. PLoS One, 2011. 6(7): p. e23035.

11. Salonen, A., W.M. de Vos, and A. Palva, Gastrointestinal microbiota in irritable bowel syndrome: present state and perspectives. Microbiology, 2010. 156(Pt 11): p. 3205-15.

12. Saulnier, D.M., et al., Gastrointestinal microbiome signatures of pediatric patients with irritable bowel syndrome. Gastroenterology, 2011. 141(5): p. 1782-91.

13. Rajilic-Stojanovic, M., et al., Global and deep molecular analysis of microbiota signatures in fecal samples from patients with irritable bowel syndrome. Gastroenterology, 2011. 141(5): $p$ $1792-801$.

14. Sobhani, I., et al., Microbial dysbiosis and colon carcinogenesis: could colon cancer be considered a bacteria-related disease? Therap Adv Gastroenterol, 2013. 6(3): p. 215-29.

15. Ley, R.E., et al., Microbial ecology - Human gut microbes associated with obesity. Nature, 2006. 444(7122): p. 1022-1023.

16. Larsen, N., et al., Gut Microbiota in Human Adults with Type 2 Diabetes Differs from NonDiabetic Adults. Plos One, 2010. 5(2).

17. Sokol, H., et al., Faecalibacterium prausnitzii is an anti-inflammatory commensal bacterium identified by gut microbiota analysis of Crohn disease patients. Proc Natl Acad Sci U S A, 2008. 105(43): p. 16731-6.

18. Alam, M.T., et al., Microbial imbalance in inflammatory bowel disease patients at different taxonomic levels. Gut Pathogens, 2020. 12(1).

19. Derrien, M., et al., Modulation of Mucosal Immune Response, Tolerance, and Proliferation in Mice Colonized by the Mucin-Degrader Akkermansia muciniphila. Front Microbiol, 2011. 2: p. 166.

20. Derrien, M., et al., Akkermansia muciniphila gen. nov., sp. nov., a human intestinal mucindegrading bacterium. Int J Syst Evol Microbiol, 2004. 54(Pt 5): p. 1469-76.

21. Kerckhoffs, A.P.M., et al., Lower Bifidobacteria counts in both duodenal mucosa-associated and fecal microbiota in irritable bowel syndrome patients. World Journal of Gastroenterology, 2009. 15(23): p. 2887-2892.

22. Balsari, A., et al., The fecal microbial population in the irritable bowel syndrome. Microbiologica, 1982. 5(3): p. 185-94.

23. Tana, C., et al., Altered profiles of intestinal microbiota and organic acids may be the origin of symptoms in irritable bowel syndrome. Neurogastroenterology and Motility, 2010. 22(5): p. 512-+.

24. Karri, S., V. Acosta-Martinez, and G. Coimbatore, Effect of dihydrotestosterone on gastrointestinal tract of male Alzheimer's disease transgenic mice. Indian Journal of Experimental Biology, 2010. 48(5): p. 453-465.

25. Braak, H., et al., Idiopathic Parkinson's disease: possible routes by which vulnerable neuronal types may be subject to neuroinvasion by an unknown pathogen. J Neural Transm (Vienna), 2003. 110(5): p. 517-36. 
26. Scher, J.U. and S.B. Abramson, The microbiome and rheumatoid arthritis. Nat Rev Rheumatol, 2011. 7(10): p. 569-78.

27. Zhang, M., et al., Analysis of gut microbiota profiles and microbe-disease associations in children with autism spectrum disorders in China. Sci Rep, 2018. 8(1): p. 13981.

28. Liu, F., et al., Altered composition and function of intestinal microbiota in autism spectrum disorders: a systematic review. Transl Psychiatry, 2019. 9(1): p. 43.

29. De Angelis, M., et al., Autism spectrum disorders and intestinal microbiota. Gut Microbes, 2015. 6(3): p. 207-13.

30. Viggiano, D., et al., Gut barrier in health and disease: focus on childhood. Eur Rev Med Pharmacol Sci, 2015. 19(6): p. 1077-85.

31. Turner, J.R., Intestinal mucosal barrier function in health and disease. Nat Rev Immunol, 2009. 9(11): p. 799-809.

32. Quigley, E.M., Leaky gut - concept or clinical entity? Curr Opin Gastroenterol, 2016. 32(2): p. 74-9.

33. Pol, O., B. Sanchez, and M.M. Puig, Peripheral effects of opioids in a model of intestinal inflammation in mice. Pharmacology, 1996. 53(6): p. 340-50.

34. Onore, C., M. Careaga, and P. Ashwood, The role of immune dysfunction in the pathophysiology of autism. Brain Behav Immun, 2012. 26(3): p. 383-92.

35. Mujagic, Z., et al., Small intestinal permeability is increased in diarrhoea predominant IBS, while alterations in gastroduodenal permeability in all IBS subtypes are largely attributable to confounders. Aliment Pharmacol Ther, 2014. 40(3): p. 288-97.

36. Zhou, Q., et al., MicroRNA-29a regulates intestinal membrane permeability in patients with irritable bowel syndrome. Gut, 2010. 59(6): p. 775-84.

37. Zhou, Q., B. Zhang, and G.N. Verne, Intestinal membrane permeability and hypersensitivity in the irritable bowel syndrome. Pain, 2009. 146(1-2): p. 41-6.

38. Drossman, D.A., The functional gastrointestinal disorders and the Rome III process. Gastroenterology, 2006. 130(5): p. 1377-90.

39. Longstreth, G.F., et al., Functional bowel disorders. Gastroenterology, 2006. 130(5): p. 148091.

40. Drossman, D.A., Functional Gastrointestinal Disorders: History, Pathophysiology, Clinical Features and Rome IV. Gastroenterology, 2016.

41. Soares, R.L., Irritable bowel syndrome: a clinical review. World J Gastroenterol, 2014. 20(34): p. 12144-60.

42. Manning, A.P., et al., Towards positive diagnosis of the irritable bowel. Br Med J, 1978. 2(6138): p. 653-4.

43. Whitehead, W.E., O.S. Palsson, and M. Simren, Irritable bowel syndrome: what do the new Rome IV diagnostic guidelines mean for patient management? Expert Rev Gastroenterol Hepatol, 2017. 11(4): p. 281-283.

44. Canavan, C., J. West, and T. Card, The epidemiology of irritable bowel syndrome. Clin Epidemiol, 2014. 6: p. 71-80.

45. Drossman, D.A. and B.C. Lowman, Irritable bowel syndrome: epidemiology, diagnosis and treatment. Clin Gastroenterol, 1985. 14(3): p. 559-73.

46. Palsson, O.S., et al., Prevalence of Rome IV Functional Bowel Disorders Among Adults in the United States, Canada, and the United Kingdom. Gastroenterology, 2020. 158(5): p. 1262-+.

47. Doshi, J.A., et al., Economic burden of irritable bowel syndrome with constipation: a retrospective analysis of health care costs in a commercially insured population. J Manag Care Spec Pharm, 2014. 20(4): p. 382-90.

48. Buono, J.L., R.T. Carson, and N.M. Flores, Health-related quality of life, work productivity, and indirect costs among patients with irritable bowel syndrome with diarrhea. Health and Quality of Life Outcomes, 2017. 15.

49. Palsson, O.S., J. Baggish, and W.E. Whitehead, Episodic nature of symptoms in irritable bowel syndrome. Am J Gastroenterol, 2014. 109(9): p. 1450-60.

50. Engsbro, A.L., M. Simren, and P. Bytzer, Short-term stability of subtypes in the irritable bowel syndrome: prospective evaluation using the Rome III classification. Alimentary Pharmacology \& Therapeutics, 2012. 35(3): p. 350-359.

51. Barbara, G., et al., The Intestinal Microenvironment and Functional Gastrointestinal Disorders. Gastroenterology, 2016. 150(6): p. 1305-+.

52. Fond, G., et al., Anxiety and depression comorbidities in irritable bowel syndrome (IBS): a systematic review and meta-analysis. European Archives of Psychiatry and Clinical Neuroscience, 2014. 264(8): p. 651-660. 
53. Rodino-Janeiro, B.K., et al., A Review of Microbiota and Irritable Bowel Syndrome: Future in Therapies. Adv Ther, 2018. 35(3): p. 289-310.

54. Gazouli, M., et al., Lessons learned - resolving the enigma of genetic factors in IBS. Nature Reviews Gastroenterology \& Hepatology, 2016. 13(2): p. 77-87.

55. Bohn, L., et al., Self-Reported Food-Related Gastrointestinal Symptoms in IBS Are Common and Associated With More Severe Symptoms and Reduced Quality of Life. American Journal of Gastroenterology, 2013. 108(5): p. 634-641.

56. Aguilera-Lizarraga, J., et al., Local immune response to food antigens drives meal-induced abdominal pain. Nature, 2021.

57. Bohn, L., et al., Diet Low in FODMAPs Reduces Symptoms of Irritable Bowel Syndrome as Well as Traditional Dietary Advice: A Randomized Controlled Trial. Gastroenterology, 2015. 149(6): p. 1399-+.

58. Staudacher, H.M., et al., Comparison of symptom response following advice for a diet low in fermentable carbohydrates (FODMAPs) versus standard dietary advice in patients with irritable bowel syndrome. Journal of Human Nutrition and Dietetics, 2011. 24(5): p. 487-495.

59. Hill, P., J.G. Muir, and P.R. Gibson, Controversies and Recent Developments of the LowFODMAP Diet. Gastroenterol Hepatol (N Y), 2017. 13(1): p. 36-45.

60. Eswaran, S.L., et al., A Randomized Controlled Trial Comparing the Low FODMAP Diet vs. Modified NICE Guidelines in US Adults with IBS-D. American Journal of Gastroenterology, 2016. 111(12): p. 1824-1832.

61. McKenzie, Y.A., et al., British Dietetic Association systematic review and evidence-based practice guidelines for the dietary management of irritable bowel syndrome in adults (2016 update). Journal of Human Nutrition and Dietetics, 2016. 29(5): p. 549-575.

62. Corsetti, M., L. Van Oudenhove, and J. Tack, The quest for biomarkers in IBS-where should it lead us? Neurogastroenterology and Motility, 2014. 26(12): p. 1669-1676.

63. Lembo, A.J., et al., Use of serum biomarkers in a diagnostic test for irritable bowel syndrome. Alimentary Pharmacology \& Therapeutics, 2009. 29(8): p. 834-842.

64. Baumgart, D.C. and W.J. Sandborn, Crohn's disease. Lancet, 2012. 380(9853): p. 1590-605.

65. Burisch, J., et al., The burden of inflammatory bowel disease in Europe. Journal of Crohns \& Colitis, 2013. 7(4): p. 322-337.

66. van den Heuvel, T.R.A., et al., A 20-Year Temporal Change Analysis in Incidence, Presenting Phenotype and Mortality, in the Dutch IBDSL Cohort-Can Diagnostic Factors Explain the Increase in IBD Incidence? J Crohns Colitis, 2017. 11(10): p. 1169-1179.

67. Jeuring, S.F.G., et al., Epidemiology and Long-term Outcome of Inflammatory Bowel Disease Diagnosed at Elderly AgeAn Increasing Distinct Entity? Inflammatory Bowel Diseases, 2016. 22(6): p. 1425-1434.

68. Tsianos, E.V., K.H. Katsanos, and V.E. Tsianos, Role of genetics in the diagnosis and prognosis of Crohn's disease. World J Gastroenterol, 2011. 17(48): p. 5246-59.

69. Graff, L.A., et al., The relationship of inflammatory bowel disease type and activity to psychological functioning and quality of life. Clin Gastroenterol Hepatol, 2006. 4(12): p. 14911501.

70. Boye, B., et al., INSPIRE study: does stress management improve the course of inflammatory bowel disease and disease-specific quality of life in distressed patients with ulcerative colitis or Crohn's disease? A randomized controlled trial. Inflamm Bowel Dis, 2011. 17(9): p. 186373.

71. Lichtenstein, G.R., et al., Management of Crohn's disease in adults. Am J Gastroenterol, 2009. 104(2): p. 465-83; quiz 464, 484.

72. Boyapati, R., J. Satsangi, and G.T. Ho, Pathogenesis of Crohn's disease. F1000Prime Rep, 2015. 7: p. 44.

73. Bodelier, A.G.L., et al., Volatile Organic Compounds in exhaled air as novel marker for disease activity in Crohn's disease: A metabolomic approach. , in Inflammatory Bowel Disease.

74. Regueiro, M., et al., Crohn's disease activity index does not correlate with endoscopic recurrence one year after ileocolonic resection. Inflamm Bowel Dis, 2011. 17(1): p. 118-26.

75. Bodelier, A.G., et al., Volatile Organic Compounds in Exhaled Air as Novel Marker for Disease Activity in Crohn's Disease: A Metabolomic Approach. Inflamm Bowel Dis, 2015. 21(8): p. 1776-85.

76. Buriani, A., et al., Omic techniques in systems biology approaches to traditional Chinese medicine research: present and future. J Ethnopharmacol, 2012. 140(3): p. 535-44. 
77. Hasin, Y., M. Seldin, and A. Lusis, Multi-omics approaches to disease. Genome Biol, 2017. 18(1): p. 83.

78. Goldsmith, P., et al., Metabonomics: a useful tool for the future surgeon. J Surg Res, 2010. 160(1): p. 122-32.

79. Fiehn, O., Metabolomics--the link between genotypes and phenotypes. Plant Mol Biol, 2002. 48(1-2): p. 155-71.

80. Smolinska, A., et al., NMR and pattern recognition methods in metabolomics: from data acquisition to biomarker discovery: a review. Anal Chim Acta, 2012. 750: p. 82-97.

81. Nicholson, J.K. and J.C. Lindon, Systems biology - Metabonomics. Nature, 2008. 455(7216): p. 1054-1056.

82. Rowan, D.D., Volatile metabolites. Metabolites, 2011. 1(1): p. 41-63.

83. Pichersky, E. and J. Gershenzon, The formation and function of plant volatiles: perfumes for pollinator attraction and defense. Curr Opin Plant Biol, 2002. 5(3): p. 237-43.

84. Vickers, C.E., et al., $A$ unified mechanism of action for volatile isoprenoids in plant abiotic stress. Nat Chem Biol, 2009. 5(5): p. 283-91.

85. Pierce, S.K., H.L. Gearhart, and D. Payne-Bose, The analysis of human breath and urine for organic components with chromatographic and mass spectrometric techniques: A review. Talanta, 1977. 24(8): p. 473-81.

86. Ashley, D.L., et al., Measurement of volatile organic compounds in human blood. Environ Health Perspect, 1996. 104 Suppl 5: p. 871-7.

87. Garner, C.E., et al., Volatile organic compounds from feces and their potential for diagnosis of gastrointestinal disease. Faseb Journal, 2007. 21(8): p. 1675-1688.

88. Garner, C.E., et al., Volatile organic compounds from feces and their potential for diagnosis of gastrointestinal disease. FASEB J, 2007. 21(8): p. 1675-88.

89. Soini, H.A., et al., Analysis of volatile organic compounds in human saliva by a static sorptive extraction method and gas chromatography-mass spectrometry. J Chem Ecol, 2010. 36(9): p. 1035-42.

90. Popov, T.A., Human exhaled breath analysis. Ann Allergy Asthma Immunol, 2011. 106(6): p. 451-6; quiz 457.

91. Smolinska, A., et al., Current breathomics--a review on data pre-processing techniques and machine learning in metabolomics breath analysis. J Breath Res, 2014. 8(2): p. 027105.

92. Miekisch, W., J.K. Schubert, and G.F. Noeldge-Schomburg, Diagnostic potential of breath analysis--focus on volatile organic compounds. Clin Chim Acta, 2004. 347(1-2): p. 25-39.

93. Laffel, L., Ketone bodies: a review of physiology, pathophysiology and application of monitoring to diabetes. Diabetes Metab Res Rev, 1999. 15(6): p. 412-26.

94. Barnett, D., C.N. Tassopoulos, and T.R. Fraser, Breath acetone and blood sugar measurements in diabetes. Clin Sci, 1969. 37(2): p. 570.

95. Karl, T., et al., Human breath isoprene and its relation to blood cholesterol levels: new measurements and modeling. J Appl Physiol (1985), 2001. 91(2): p. 762-70.

96. Turner, C., P. Spanel, and D. Smith, A longitudinal study of breath isoprene in healthy volunteers using selected ion flow tube mass spectrometry (SIFT-MS). Physiol Meas, 2006. 27(1): p. 13-22.

97. Ratcliffe, N., et al., A mechanistic study and review of volatile products from peroxidation of unsaturated fatty acids: an aid to understanding the origins of volatile organic compounds from the human body. J Breath Res, 2020. 14(3): p. 034001.

98. Kneepkens, C.M., G. Lepage, and C.C. Roy, The potential of the hydrocarbon breath test as a measure of lipid peroxidation. Free Radic Biol Med, 1994. 17(2): p. 127-60.

99. Van Berkel, J.J., et al., Development of accurate classification method based on the analysis of volatile organic compounds from human exhaled air. Journal of chromatography. B, Analytical technologies in the biomedical and life sciences, 2008. 861(1): p. 101-7.

100. Baranska, A., et al., Dynamic collection and analysis of volatile organic compounds from the headspace of cell cultures. J Breath Res, 2015. 9(4): p. 047102.

101. Bos, L.D., P.J. Sterk, and M.J. Schultz, Volatile metabolites of pathogens: a systematic review. PLoS Pathog, 2013. 9(5): p. e1003311.

102. Boots, A.W., et al., Identification of microorganisms based on headspace analysis of volatile organic compounds by gas chromatography-mass spectrometry. J Breath Res, 2014. 8(2): p. 027106.

103. Taylor, C., et al., Analysis of Listeria using exogenous volatile organic compound metabolites and their detection by static headspace-multi-capillary column-gas chromatography-ion 
mobility spectrometry (SHS-MCC-GC-IMS). Anal Bioanal Chem, 2017. 409(17): p. 42474256.

104. Nizio, K.D., et al., In vitro volatile organic compound profiling using GCXGC-TOFMS to differentiate bacteria associated with lung infections: a proof-of-concept study. J Breath Res, 2016. 10(2): p. 026008.

105. Filipiak, W., et al., TD-GC-MS analysis of volatile metabolites of human lung cancer and normal cells in vitro. Cancer Epidemiol Biomarkers Prev, 2010. 19(1): p. 182-95.

106. Filipiak, W., et al., Release of volatile organic compounds (VOCs) from the lung cancer cell line CALU-1 in vitro. Cancer Cell Int, 2008. 8: p. 17.

107. Mochalski, P., et al., Release and uptake of volatile organic compounds by human hepatocellular carcinoma cells (HepG2) in vitro. Cancer Cell Int, 2013. 13(1): p. 72.

108. Sponring, A., et al., Analysis of volatile organic compounds (VOCs) in the headspace of NCIH1666 lung cancer cells. Cancer Biomark, 2010. 7(3): p. 153-61.

109. Sponring, A., et al., Release of volatile organic compounds from the lung cancer cell line NClH2087 in vitro. Anticancer Res, 2009. 29(1): p. 419-26.

110. Wang, Y., et al., The analysis of volatile organic compounds biomarkers for lung cancer in exhaled breath, tissues and cell lines. Cancer Biomark, 2012. 11(4): p. 129-37.

111. de Lacy Costello, B., et al., A review of the volatiles from the healthy human body. J Breath Res, 2014. 8(1): p. 014001.

112. Boots, A.W., et al., The versatile use of exhaled volatile organic compounds in human health and disease. J Breath Res, 2012. 6(2): p. 027108. 


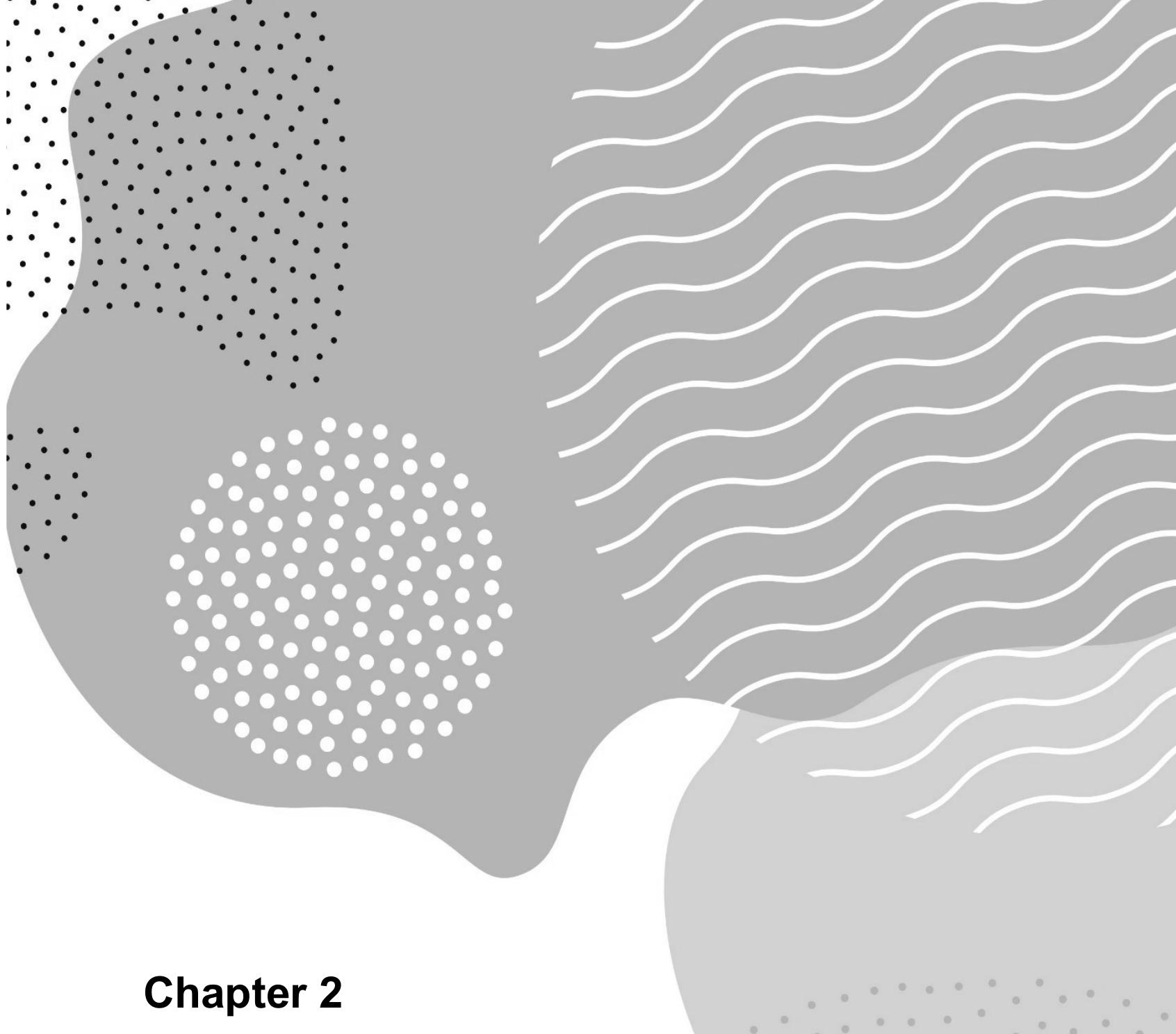

Factors that influence the volatile organic compound content in human breath

L. Blanchet, A. Smolinska, A. Baranska, E. Tigchelaar, M. Swertz, A. Zhernakova, J.W. Dallinga, C. Wijmenga, F. J. van Schooten

Journal of Breath Research 11 (2017) 016013 


\section{Abstract}

Background: Thousands of endogenous and exogenous volatile organic compounds (VOCs) are excreted in each breath. Inflammatory and deviant metabolic processes affect the level of endogenous VOCs, which can serve as specific biomarkers for clinical diagnosis and disease monitoring. Important issues that still need to be tackled are related to potential confounding factors like gender and age and endogenous and exogenous factors, like smoking.

Methods: The aim of this study was to systematically access the effect of endogenous and exogenous factors on VOC composition of exhaled breath. In the current study breath samples from 1417 adult participants from the LifeLines cohort, a general population cohort in the Netherlands, were collected and the total content of VOCs was measured using Gas Chromatography-time-of-flight-Mass Spectrometry. Breath samples were collected in Groningen and transferred to carbon tubes immediately. These samples were then shipped to Maastricht and measured in batches. VOCs profiles were correlated to 14 relevant characteristics of all participants including age, BMI, smoking and blood cell counts and metabolic parameters as well as to 16 classes of medications.

Results: VOCs profiles were shown to be significantly influenced by smoking behavior and to a lesser extent by age, BMI and gender. These factors need to be controlled for in breath analysis studies. We found no evidence whatsoever in this 1417 subjects' cohort that white blood cell counts, cholesterol or triglycerides levels have an influence on the VOC profile. Thus, they may not have to be controlled for in exhaled breath studies.

Conclusion: The large cohort of volunteers used here represents a unique opportunity to gauge the factors influencing VOCs profiles in a general population i.e., the most clinically relevant population. Classical clinical parameters and smoking habits clearly influence breath content and should therefore be accounted for in future clinical studies involving breath analysis. 


\section{Introduction}

With each single breath, humans exhale hundreds of different molecules [1, 2]. These volatile molecules are excreted via the lungs but originate from multiple sources: exposure to ambient air, lung tissues, bacterial infections, blood stream. Via the latter, Volatile Organic Compounds (VOCs) in exhaled air are reflecting the overall metabolism and thus can be influenced by key players such as liver metabolism, nutrition, and disease activity. Sampling exhaled breath is completely non-invasive, and it can be performed very frequently and rapidly. For these reasons' breath analysis has a tremendous potential for clinical diagnosis and monitoring [35].

Respiratory diseases are intuitively related to exhaled air analysis. Indeed, breath VOCs content has been shown to be affected by respiratory diseases such as asthma [6-9], cystic fibrosis [10, 11], COPD [12-15] and tuberculosis [16-19]. Further efforts have been invested in detecting infectious diseases [20-23] and identifying VOCs specific to pathogenic bacteria [24-27]. Also, lung cancer has been a topic of interest since the early days of breath analysis [28-30]. Next to the respiratory tract exhaled air also carries information from other internal organs. Through oxidative stress, breath markers have been related to cardiac diseases [31, 32]. Candidate biomarkers in breath have been reported for breast cancer [33] and liver diseases [34] such as NASH [35-37]. Gastro-intestinal diseases such as Inflammatory Bowel Diseases [38-42] and more specifically Crohn's disease [43, 44] can be detected and monitored via analysis of exhaled air.

Any application of exhaled air to diagnostics is confronted with the question of possible confounders. To evaluate the potential of e.g., a breath biomarker adequately, one should know the influence of possible confounders on exhaled air content. The effect of smoking, an obvious factor influencing breath content, has been demonstrated several times previously. Thus, each study involving breath air analysis takes smoking habit into account during the process of study design. Numerous activities may result in high levels of exogenous compounds in exhaled air, e.g. high-exposure professions [45], the use of consumer products (make-up, 
soaps, household cleaners, and pesticides), or even showering with city water (halogens [46-48]). Previous and current uptake of environmental air has been demonstrated as a factor influencing the content of exhaled air if used for diagnostic purposes [49, 50]. In a recent publication by Das et al. [51] gender differences were demonstrated in excreted VOCs profiles measured by two-dimensional Gas Chromatography-time-of-flight Mass Spectrometry. However, until now many other parameters, such as age, Body Mass Index (BMI), blood and biomedical parameters, physical health and medications have not been considered and tested as possible confounders in breath analysis.

The objective of this study is to evaluate the influence of possible confounders in breath analysis and to identify the associated molecules. We take advantage of the unique opportunities offered by the LifeLines cohort [52]: the high number of volunteers increase the statistical power, thus allowing us to investigate subtle changes such as variations in VOCs levels in relation to clinical parameters. The objective of this general population screening is to study ageing, disease development, and general well-being using (epi)genetic, biomedical, environmental, and psychosocial tools. A subset of this cohort consisting of 1417 individuals [53] has been selected for a more exhaustive characterization in relation to healthy ageing, disease development and general well-being. This subcohort (LifeLines Detailed Extensive Examination of Participants (LL DEEP) allows for the estimation of the influence on breath content of various factors such as age, gender, BMI and physical health but also of more specific ones such as blood parameters, as represented in Figure 1. Such influence can be characterized in two distinct ways. One can search for statistically significant differences between groups e.g., males versus females. In such a situation, classical hypothesis testing (i.e., involving the test of a null hypothesis against an alternative hypothesis) is suitable. However, the existence of a statistical difference between means (as usually tested e.g., by t-test) does not necessarily imply that the total population can really be divided into subgroups. A more critical question to answer to is: does the factor lead to subdivisions of the total population? From now on, we will distinguish between the classical statistical difference (i.e., statistical testing) and the stronger case of discrimination 
(i.e., subdivision into groups). We demonstrate that breath content, indeed, reflects our metabolic status and that often the usual biological confounders are not playing a critical role in the variation observed in breath analysis.

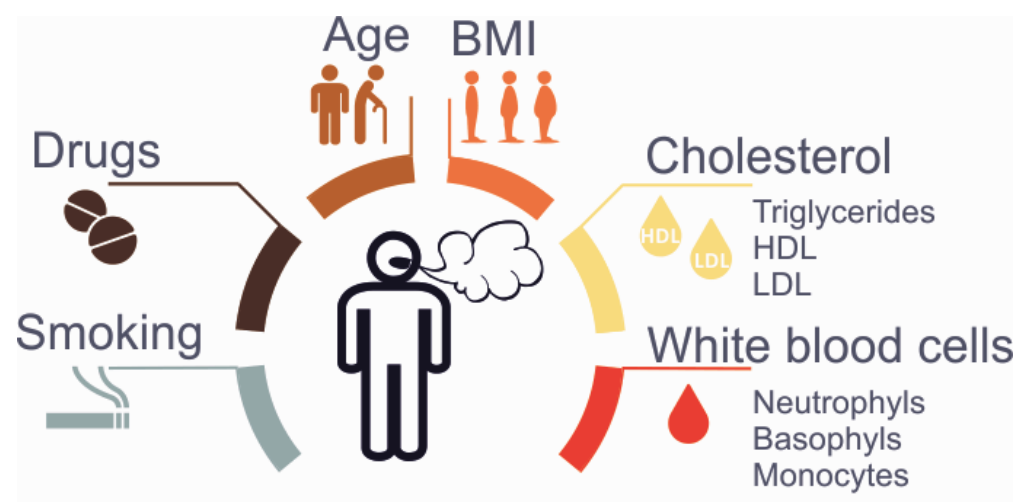

Figure 1. Exogenous and endogenous factors potentially affecting VOCs concentration in breath.

\section{Materials and Methods}

A detailed description of the material and methods is provided in the supplementary information.

\section{Cohort description (demographics)}

LL DEEP is a population-based prospective cohort. Part of this cohort encompasses individuals before the onset of any disease, allowing for (early) biomarkers discovery. First, individuals aged 25-50 years old are invited by their general practitioner to participate in the longitudinal LifeLines study. Upon inclusion, family members of the participants are also invited to participate. From April to August 2013, a subset of 1417 participants was selected for a more thorough screening: LL DEEP [53]. LL DEEP is not investigating longitudinal aspects but rather dives in a more exhaustive molecular screening. LL DEEP original purpose is to focus on the characterization of gastrointestinal-health-related problems like irritable bowel syndrome. Three additional tubes of blood and exhaled air were collected during the 
participant's visit to the LifeLines site. Participants' characteristics are presented in Table 1.

The LifeLines DEEP study was approved by the ethics committee of the University Medical Centre Groningen (document number METC UMCG LLDEEP: M12.113965). All participants signed an informed consent prior to enrolment.

\begin{tabular}{|c|c|}
\hline Factor & Distribution \\
\hline Gender & $58 \%(822)+42 \%(521)$ ○ \\
\hline Age & $46 \pm 20$ \\
\hline BMI & $24.5 \pm 5.0$ \\
\hline Smoking habits & $\begin{array}{c}20 \%(284) \text { Smokers, } \\
31 \% \text { (439) Ex-smokers, } \\
49 \% \text { (694) Non-smokers }\end{array}$ \\
\hline Total Cholesterol (mmol/l) & $4.9 \pm 1.4$ \\
\hline HDL (mmol/l) & $1.5 \pm 0.6$ \\
\hline LDL (mmol/l) & $3.0 \pm 1.2$ \\
\hline Triglycerides (mmol/l) & $0.94 \pm 0.67$ \\
\hline Neutrophils (10 billion/l) & $2.83 \pm 1.28$ \\
\hline Basophils (10 billion/l) & $0.3 \pm 0.4$ \\
\hline Monocytes (10 billion/l) & $0.48 \pm 0.18$ \\
\hline Total white blood cells (10 billion/l) & $5.4 \pm 1.9$ \\
\hline Contraception & $29 \%(238)$ of $q$ \\
\hline
\end{tabular}

Table 1. Demographics and other characteristics of the $L L D E E P$ cohort. Values reported are the median \pm interquartile range. 


\section{Sampling and breath analysis}

Volunteers breathe through a mouthpiece connected to the valve of a resistancefree 3L Tedlar bag (SKC Ltd, Dorset, UK). The content of the plastic bags is transferred using a pump with constant flow to a stainless-steel two-bed sorption tube filled with carbograph 1TD/Carbopack X (Markes International, Llantrisant, Wales, UK), within $1 \mathrm{~h}$ after collection. The breath samples were measured in batches within 2 weeks after collection by gas chromatography-time of flight-mass spectrometry (GC-tof-MS) [54]. The volatile compounds were first released from the sorption tubes by thermal desorption and loaded onto a cold sorption trap, from which they were injected on the column for the GC-tof-MS analysis. The temperature of the GC (Thermo Fischer Scientific, Austin, USA, column: Restek RTX-5ms, $30 \mathrm{~m} \times 0.25 \mathrm{~mm} \mathrm{ID}$, coated with $1.0 \mu \mathrm{m} \mathrm{HP}-5$ phase) was programmed as follows: first $40^{\circ} \mathrm{C}$ for 5 minutes then it was increased by $10^{\circ} \mathrm{C} / \mathrm{min}$ until $270^{\circ} \mathrm{C}$. This temperature was maintained for 5 minutes. Electron ionization at $70 \mathrm{eV}$ was used with $5 \mathrm{~Hz}$ scanning rate over a mass range of 35-350.

\section{Data analysis}

Before the statistical analysis, the raw GC-tof-MS data were preprocessed [55, 56]. A list of chromatographic peaks was established for each chromatogram. The final data table summarized the information acquired on 1417 individuals and 678 chemical compounds. To be retained a VOC has to be present in at least $20 \%$ of the samples. Probabilistic quotient normalization was applied [57] to suppress any effect related to the different dilution of the samples. Note that this normalization step does not correct for completely missing peaks/compounds. To complete the final data table batch differences as a result of variations in sampling and/or instrumental performance are corrected for and outliers are detected and subsequently removed. More details are provided in supplementary information.

Multivariate Analysis Of Variance (MANOVA) was applied for statistical hypotheses testing. When the number of studied compounds is much larger than the number of available individuals (e.g., women taking contraception) a regularized version of MANOVA (rMANOVA) is employed [58]. To estimate p-values we evaluated the 
distribution of the test statistic using the sequential permutation test strategy [59]. The maximal number of permutations was set to 1000 . Note that for practical reasons we limit ourselves to the study of the main effects and do not consider any possible interactions terms (e.g., gender-neutrophils level interaction). If a difference between groups is detected, it can be visualized using a score plot of the canonical variates (CVs) estimated by MANOVA. A canonical variate represents the linear combinations of the measured VOCs which maximize the difference between the means of the tested groups. Discriminant analysis was performed using Random Forests (RF) [60]. All calculations were performed using Matlab R2013a (Statistics Toolbox).

The putative chemical identification of the most relevant VOCs was done based on spectrum recognition using the National Institute of Standards and Technology (NIST) mass spectral library. This automated identification is further checked by an experienced mass-spectrometrist taking into account both mass spectra and retention times.

\section{Results and Discussion}

We observed that gender, age, BMI and smoking significantly affect the breath profile but only smokers vs non-smokers lead to a clear separation of the population into (non-overlapping) groups. All results are summarized in Table 2.

\section{Smokers vs non-smokers}

Samples were assigned to one of the following three groups: non-smokers $(n=520)$, smokers $(n=318)$ and ex-smokers $(n=198)$. As previously demonstrated, smoking behavior is expected to affect the exhaled air profile. Therefore, this factor of influence can be considered as a positive control for our approach. Here, in accordance with previous results, smoking is detected as an influencing factor by both statistical techniques, i.e., MANOVA $(p<0.001)$ and RF discrimination model. Figure 2A presents the score plot for the influence of smoking behavior. Each point represents an individual breath profile and is color-coded according to the smoking 
habits. Three clouds of points are clearly formed and separated from each other indicating significant differences among their means (i.e., the center of the clouds).

\begin{tabular}{lcc}
\hline Factor of influence & p-value & Discrimination power \\
\hline Gender & $<0.001$ & - \\
\hline Age & 0.01 & - \\
\hline BMI & $<0.001$ & - \\
\hline Smoking habits & $<0.001$ & 0.84 \\
\hline Total Cholesterol (mmol/l) & NS & - \\
\hline HDL (mmol/l) & NS & - \\
\hline LDL (mmol/l) & NS & - \\
\hline Triglycerides (mmol/l) & NS & - \\
\hline Neutrophils (10 billion/l) & NS & - \\
\hline Eosinophils (10 billion/l) & NS & - \\
\hline Basophils (10 billion/l) & NS & - \\
\hline Monocytes (10 billion/l) & NS & - \\
\hline Total white blood cells (10 billion/l) & NS & \\
\hline Contraception & NS & - \\
\hline
\end{tabular}

Table 2. Evaluation of the influence of possible confounders on breath content.

Next, we examine the individual contribution of each VOC to the observed groups' difference. The multivariate pattern corresponding to the difference between smokers and non-smokers is dominated by few compounds, reported in Table 3 and with more details in supplementary Table S1 and S2. Figure S1 allows estimating the relative importance of the VOCs and whether they are either elevated or decreased in a specific group. The relative abundances of the VOCs and their appearance in the total cohort are included in supplementary materials in Table S2. Briefly, among the selected VOCs furan and trichloroethylene are known to be present in mainstream cigarette smoke, thus their relative abundances are elevated 
in smokers. We also found the pyridine level to be more elevated in smokers, in line with previous findings [61].

The separation observable in Figure 2A already gives a hint that the smokers population is not only differing in its average profile (as tested by MANOVA) but that it can be seen as a separate sub-population. This is confirmed by the results obtained using RF. An RF model trained to distinguish smoker from non-smokers is able to classify correctly $82 \%$ of a (blind) test set, this corresponds to an Area Under the Receiver Operating Characteristic (AUROC) of 0.84 .

\section{Gender}

Gender affects metabolism, hence one could expect differences in breath content as well. As reported in Table 2, our analysis confirms that a significant statistical difference can be detected between the mean VOC profiles of male and female ( $n=$ 562 and 774 respectively, $p<0.001$ ). Similarly, to the smoking habits, a score plot of $\mathrm{CV}$ s can be used to visualize the separation between genders. Although the VOC profiles of male and female are different, the separation between the two groups visible in Figure 2B is not very marked. Indeed, this difference is not sufficient to discriminate males and females. Using RF modelling it is not possible to predict the gender of volunteers based on their VOCs profiles. This means that the difference existing between men and women breath is not strong enough to create two completely distinct groups. Note that both men and women populations contain the same proportions of smokers (approx. $20 \%$ ).

We next selected the set of compounds that were significantly different between male and female subjects using MANOVA. Among the important compounds, toluene is likely to be related to exposure [45]. Interestingly the metabolism of toluene has been reported as gender dependent in rats. Carbon disulfide is also selected. This small molecule is typically originating from environmental exposure to pollution $[62,63]$. However, epidemiological study has demonstrated that males are in average more exposed to it due to their professional activities. Interestingly, a study performed in rats showed that females metabolized toluene faster than males [64]. Note that $\mathrm{CS}_{2}$ can also be formed in gut by the microbiome [65]. 


\begin{tabular}{|c|c|c|c|}
\hline & $\begin{array}{l}\text { Retention } \\
\text { time (min) }\end{array}$ & Identification & $\begin{array}{c}\text { Change in } \\
\text { concentration }\end{array}$ \\
\hline \multirow{5}{*}{ 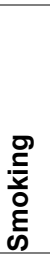 } & 7.04 & Trichloroethylene & $\uparrow$ \\
\hline & 8.38 & Pyridine & $\uparrow$ \\
\hline & 9.07 & 3-methylheptane & $\uparrow$ \\
\hline & 11.71 & 2-ethyl- or 3-ethylfuran & $\uparrow$ \\
\hline & 14.80 & Unknown & $\uparrow$ \\
\hline \multirow{5}{*}{ 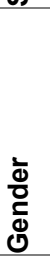 } & 2.98 & Carbon disulfide (CS2) & $\downarrow$ \\
\hline & 3.83 & Unknown & $\downarrow$ \\
\hline & 8.38 & Pyridine & $\uparrow$ \\
\hline & 9.07 & Toluene & $\uparrow$ \\
\hline & 15.29 & 2,3-dimethyl-4-penten-ol & $\downarrow$ \\
\hline \multirow[b]{15}{*}{$\sum_{\boldsymbol{m}}$} & 2.61 & Dimethylsulfide & $\downarrow$ \\
\hline & 9.82 & 2-hexanone & $\uparrow$ \\
\hline & 11.46 & m-/p-xylene & $\uparrow$ \\
\hline & 12.10 & o-xylene & $\uparrow$ \\
\hline & 13.52 & 1,2,4-trimethylbenzene & $\downarrow$ \\
\hline & 13.87 & 7-methyl-3-methylene-1,6-octadiene & $\uparrow$ \\
\hline & 14.00 & 3-formylthiophene & $\downarrow$ \\
\hline & 14.63 & 2-acetylpyrrole & $\uparrow$ \\
\hline & 14.65 & 3-pyridinemethanol & $\downarrow$ \\
\hline & 15.29 & Phenyl acetate & $\uparrow$ \\
\hline & 16.48 & Unknown & $\downarrow$ \\
\hline & 17.44 & Unknown & $\uparrow$ \\
\hline & 18.94 & 1-methoxy-4-(1-propenyl)-benzene & $\downarrow$ \\
\hline & 19.34 & 1-methoxy-4-(2-propenyl)-benzene & $\downarrow$ \\
\hline & 22.73 & 2-phenoxyphenol & $\downarrow$ \\
\hline \multirow[b]{8}{*}{ 囱 } & 2.58 & Isoprene & $\downarrow$ \\
\hline & 2.61 & Dimethylsulfide & $\uparrow$ \\
\hline & 8.38 & Pyridine & $\downarrow$ \\
\hline & 11.63 & 1,2,4-trimethylcyclohexane & $\uparrow$ \\
\hline & 18.38 & Unknown & $\downarrow$ \\
\hline & 18.68 & 2-methyl-5-isopropenyl-2-cyclohexen-1- & $\downarrow$ \\
\hline & 19.34 & 1-methoxy-4-(2-propenyl)-benzene & $\uparrow$ \\
\hline & 21.66 & Dodecanal & $\downarrow$ \\
\hline
\end{tabular}

\# $\uparrow$ indicates higher in smokers, higher in men, higher in high BMI, higher in high age

Table 3. Selected VOCs driving the differences between the groups stratified according to smoking behavior, gender, BMI and age. Arrows indicate if the level of VOC is upregulated in respectively in smoker group, in males, in lower BMI and younger volunteers 


\section{Body Mass Index (BMI)}

$\mathrm{BMI}$ is a continuous variable. It is, therefore, possible to analyse the impact of BMI on exhaled air in two distinct ways. A regression model can evaluate the relation between VOCs and BMI as a continuum, or the BMI can be stratified. The second option has the advantage of simplifying the analysis and corresponds more to the clinical use of the BMI, which divides volunteers in groups such as underweighted, normal weight, overweight and obese. Since in our approach we choose to search for statistically significant and discriminatory volatiles in exhaled air, we opt for the second route. In that way, the same two methods, MANOVA and RF, are used along the whole study.

We divided the measurements available in three groups: Normal BMI (i.e., below 25, $n=631$ ), overweight (BMI between 25 and 30, n=351) and obese (BMI over 30, n= 129). Only two individuals were underweight (BMI < 18.5). A statisticaly significant difference can be detected between the average breath profiles of the threegroups $(p<0.001)$. Note that a valid regression model using the exact BMI values could not be obtained. Various VOCs involved in this difference could be related to food, with a number of food flavouring compounds (e.g., 7-methyl-3-methylene-1,6-octadiene, 2-acetylpyrrole or 1-methoxy-4-(1-propenyl)-benzene) or downstream products of artificial sweeteners (Phenylacetate). The observed differences could result from the different diet of the groups or from direct metabolic differences or both.

\section{Age}

The impact of ageing on metabolism is a subject discussed extensively in the literature. One can reasonably expect that such a factor influences the concentrations of a number of metabolites and eventually VOCs in exhaled air. Here the age range was divided in segments of ten years. Volunteers in their twenties form one group, the ones in their thirties, and so on. The groups are used to evaluate the impact of ageing on the breath profiles using MANOVA. A significant difference is detected $(p=0.002)$. Figure $2 \mathrm{D}$ shows that interestingly the ageing seems to follow a specific direction until approx. 60s and then rotates towards another direction. Although statistically significant differences of means are detected, the effects are 
too weak to lead to a discriminative model. The MANOVA model points towards VOCs related to aging. One of them is isoprene, which was previously related to age and is among the most important VOCs. Methionine metabolism is known to be involved in aging. One of the significant compounds, dimethylsulfide, is a downstream product of methionine.
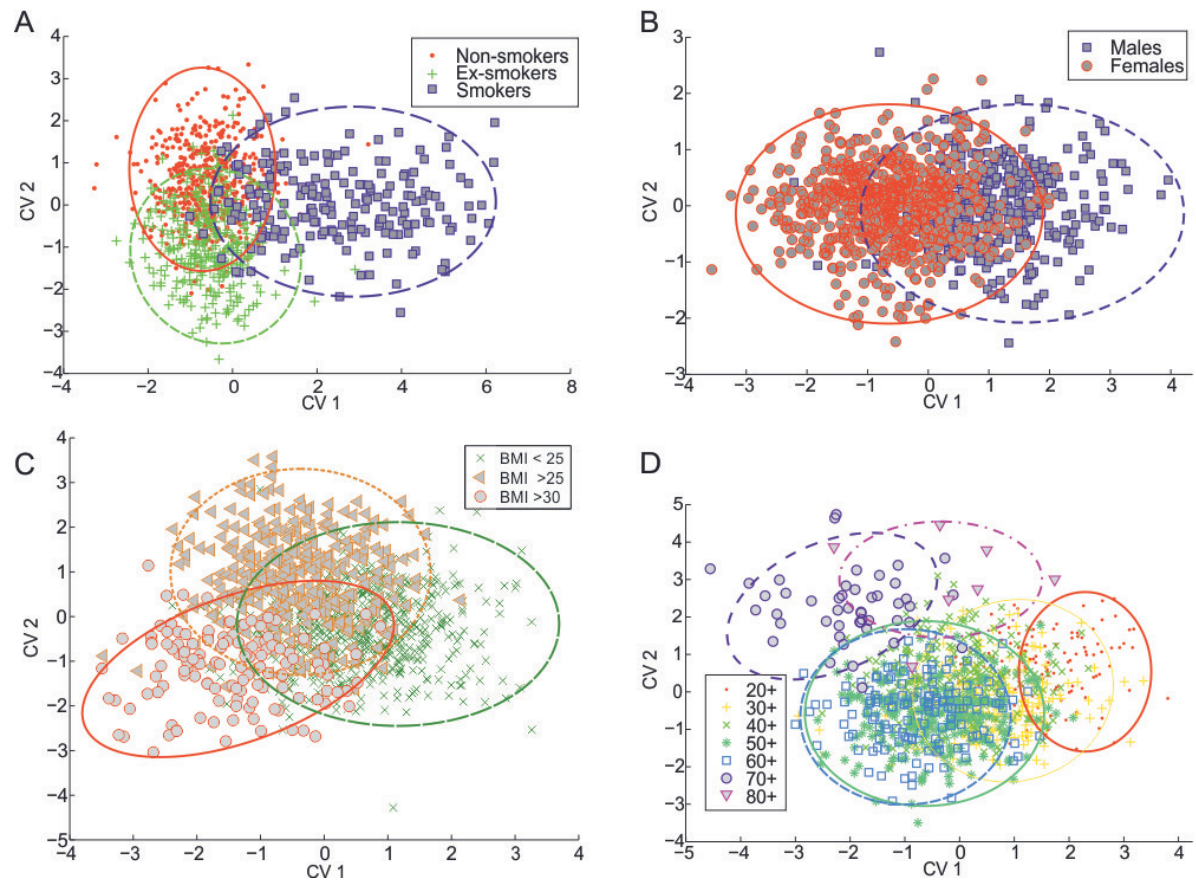

Figure 2. Score plot of the canonical variates (CV) obtained from the MANOVA analyses for the impact in exhaled air of A) smoking behaviour B) gender C) BMI and D) age. Each point in the figure represents a single breath sample. $X$-axis and $y$-axis correspond to the most significant combination of the VOCs with respect to each of the confounders. All factors affect significantly ( $p$-value $\leq 0.01)$ the content of exhaled air.

\section{Cholesterol}

Cholesterol level is documented for 1046 members of the cohort in term of total cholesterol, low-density lipoprotein (LDL), high-density lipoprotein (HDL) and triglycerides. The number of patients with elevated cholesterol levels (Total cholesterol $>5.2 \mathrm{mmol} / \mathrm{L}, \mathrm{LDL}>3.5 \mathrm{mmol} / \mathrm{L}, \mathrm{HDL}<1 \mathrm{mmol} / \mathrm{L}$ ) is small in relation to the number of patients with normal values, at a ratio of approx. 1 to 10 . It is, therefore, 
necessary to balance the number of individuals with normal and abnormal levels of cholesterol for the analysis. Given the previous results, we also matched the two groups (abnormal and normal levels of cholesterol) in terms of gender, age, BMI and smoking behavior. None of the cholesterol levels were detected as statistically significant or discriminatory.

\section{White blood cells count}

The immune response is expected to influence VOC profiles. The white blood cell counts registered for 1027 LL DEEP volunteers are used to study this aspect. Surprisingly, no significance difference is detected, neither considering the total white blood cell count or its subdivisions: neutrophils, basophils, monocytes, lymphocytes. The lack of significant or discriminatory differences can be explained by lack of statistical power of the cohort, which is largely composed of healthy people. For example, the number of people with an abnormal number of neutrophils is only 11 , thus not allowing to properly test their influence on the VOCs. Within this LL DEEP cohort only a few individuals have serious medical conditions, yet none with very elevated white blood cell counts. Thus, lack of individuals with elevated levels of leukocytes is a limitation of the study and can explain the negative results obtained for this particular confounder.

\section{Contraception}

Medications are obviously influencing metabolism and thus may be suspected to influence breath content. In the LL DEEP cohort information is available on the use of drugs. However, looking into this particular effect, it is difficult in the absence of the appropriate controls: patients with the same diseases but without treatment. One exception is chemical contraception; we can compare the 254 women taking contraception to a matched cohort of women not taking any form of contraception. After taking into account age, smoking behaviour and BMI, no significant and discriminatory difference could be observed. 


\section{Possible confounders themselves confounded}

Medications are strongly suspected to be influencing breath content [66]. Most types of drugs have significant impact, as shown in Table 4. One exception is formed by the corticosteroids, yet, by sub-stratifying per drug significant effects can be detected at least for Fluticasone and Mometasone. These results, however, are themselves affected by the underlying diseases for which the drugs have been prescribed. Volunteers forming the LL DEEP cohorts come from the general population including also people with various illnesses taking appropriate medications. Therefore, the effect of medication seen in exhaled breath is a mixture of disease and consumed drugs. Thus, at this stage it is impossible to distinguish the disease effect from the treatment effect here, yet it is important to remember that medication might affect the breath profile and should be always investigated.

To properly distinguish the effect of medication and the underlying disease a group of sick treated and sick untreated individuals have to be compared. Such comparison is, however, not visible in the current set-up of the study. The LL DEEP cohort does not contain individuals that are diagnosed with a disease and do not take medication.

In the various cases discussed above a general trend can be discerned. As expected, breath is reflecting metabolism and in that respect some factors affect the VOCs profiles. At the other hand, the metabolism is also a self-regulating complex system resilient to various influences. A factor influencing the metabolism will not necessarily change the complete metabolism drastically; hence only small changes can be detected in most cases (with the exception of smoking). Considering the identified chemical compounds, the variations observed in breath are partly related to the original cause, e.g., furan coming from cigarettes, or the food additives probably related to a diet associated with a higher BMI. 


\begin{tabular}{|c|c|c|}
\hline Drug class & $\begin{array}{l}\text { Number of individuals using } \\
\text { medication }\end{array}$ & $\begin{array}{c}\text { Significance } \\
\text { level }(p)\end{array}$ \\
\hline Proton pump inhibitors & 132 & 0.01 \\
\hline Laxatives & 33 & $<0.001$ \\
\hline Corticosteroids & 136 & 0.55 \\
\hline Fluticasone & 42 & $<0.001$ \\
\hline Mometasone & 18 & $<0.001$ \\
\hline Anti-inflammatory & 246 & $<0.001$ \\
\hline Anti-diabetic & 30 & $<0.001$ \\
\hline Hypertension & 251 & $<0.001$ \\
\hline Statins & 73 & $<0.001$ \\
\hline Contraceptive & 299 & 0.03 \\
\hline Hypothyroidism & 44 & $<0.001$ \\
\hline Immunosuppressant & 5 & - \\
\hline Anti-migraine & 32 & $<0.001$ \\
\hline Anxiolytics & 79 & $<0.001$ \\
\hline Antidepressants & 55 & $<0.001$ \\
\hline Drug class (continued) & $\begin{array}{l}\text { Number of individuals using } \\
\text { medication }\end{array}$ & $\begin{array}{l}\text { Significance } \\
\text { level }(p)\end{array}$ \\
\hline $\begin{array}{l}\text { } 32 \text {-adrenergic receptor agonist (asthma } \\
\text { COPD) }\end{array}$ & 103 & $<0.001$ \\
\hline $\begin{array}{lll}\text { Non- } \beta 2 \text {-adrenergic } & \text { receptor } & \text { agonist } \\
\text { (asthma COPD) } & & \end{array}$ & 30 & $<0.001$ \\
\hline Antihistamines & 94 & 0.04 \\
\hline
\end{tabular}

Table 4. Evaluation of the influence of medications (and the underlying diseases) on breath content evaluated in subset of LL DEEP and compared to matching groups of controls. 


\section{Summary:}

- Smoking behavior is clearly influencing the breath profile, and creates clear subpopulations

- Age, gender, and BMI significantly affect breath profiles, but their effects are more subtle than smoking behaviors

- Cholesterol levels, contraception and, more surprisingly, white blood cell counts could not be related to any variation in breath profiles in this general population cohort.

- Most medications (and/or the underlying diseases) are significantly affecting breath

\section{Conclusions}

Breath analysis is an attractive approach in the field of non-invasive diagnostics and disease monitoring. The ever-increasing amount of literature reporting candidate biomarkers in breath exemplifies the interest of the scientific community. Yet all too often the results reported are based on small patient cohorts, which impact the statistical validity and virtually forbid to investigate the impact of confounders. The large cohort of volunteers used here represents a unique opportunity to gauge the factors influencing VOCs profiles in a general population i.e., the most clinically relevant population. The current study shows the significant influence of smoking, age, gender, BMI and various medications on breath content. Note, that the effect of medications can be considered as the sum of two factors: underlying disease and pharmaceutical compounds. No significant associations were found between the content of exhaled breath and total cholesterol, triglycerides, LDL and HDL levels, contraception medication, neutrophils, basophils, monocytes and total white blood cells.

\section{Acknowledgements}

This study was supported by Top Institute Food and Nutrition (TIFN GH001). Authors wish to acknowledge Jackie Dekens for collecting the breath samples. 


\section{References}

1. Amann, A., et al., The human volatilome: volatile organic compounds (VOCs) in exhaled breath, skin emanations, urine, feces and saliva. Journal of Breath Research, 2014. 8(3).

2. Costello, B.D., et al., A review of the volatiles from the healthy human body. Journal of Breath Research, 2014. 8(1).

3. Cao, W.Q. and Y.X. Duan, Breath analysis: Potential for clinical diagnosis and exposure assessment. Clinical Chemistry, 2006. 52(5): p. 800-811.

4. Boots, A.W., et al., The versatile use of exhaled volatile organic compounds in human health and disease. Journal of Breath Research, 2012. 6: p. 27108-27108.

5. Kwak, J. and G. Preti, Volatile disease biomarkers in breath: a critique. Curr Pharm Biotechnol, 2011. 12(7): p. 1067-74.

6. Klaassen, E.M.M., et al., Exhaled Biomarkers and Gene Expression at Preschool Age Improve Asthma Prediction at 6 Years of Age. American Journal of Respiratory and Critical Care Medicine, 2014. 191(2): p. 201-207.

7. Fens, N., et al., Exhaled Breath Profiling Enables Discrimination of Chronic Obstructive Pulmonary Disease and Asthma. American Journal of Respiratory and Critical Care Medicine, 2009. 180(11): p. 1076-1082.

8. Smolinska, A., et al., Profiling of volatile organic compounds in exhaled breath as a strategy to find early predictive signatures of asthma in children. PLoS One, 2014. 9(4): p. e95668.

9. Van Vliet, D., et al., Association between exhaled inflammatory markers and asthma control in children. J Breath Res, 2016. 10(1): p. 016014.

10. Robroeks, C.M.H.H.T., et al., Metabolomics of volatile organic compounds in cystic fibrosis patients and controls. Pediatric Research, 2010. 68: p. 75-80.

11. van Mastrigt, E., et al., Exhaled breath profiling using broadband quantum cascade laser-based spectroscopy in healthy children and children with asthma and cystic fibrosis. J Breath Res, 2016. 10(2): p. 026003.

12. Van Berkel, J.J.B.N., et al., A profile of volatile organic compounds in breath discriminates COPD patients from controls. Respiratory Medicine, 2010. 104: p. 557-563.

13. Christiansen, A., et al., A systematic review of breath analysis and detection of volatile organic compounds in COPD. J Breath Res, 2016. 10(3): p. 034002.

14. Gaida, A., et al., $A$ dual center study to compare breath volatile organic compounds from smokers and non-smokers with and without COPD. J Breath Res, 2016. 10(2): p. 026006.

15. Santini, G., et al., Exhaled and non-exhaled non-invasive markers for assessment of respiratory inflammation in patients with stable COPD and healthy smokers. J Breath Res, 2016. 10(1): p. 017102.

16. Kolk, A.H.J., et al., Breath analysis as a potential diagnostic tool for tuberculosis. International Journal of Tuberculosis and Lung Disease, 2012. 16(6): p. 777-782.

17. Scott-Thomas, A., M. Epton, and S. Chambers, Validating a breath collection and analysis system for the new tuberculosis breath test. J Breath Res, 2013. 7(3): p. 037108.

18. Zetola, N.M., et al., Diagnosis of pulmonary tuberculosis and assessment of treatment response through analyses of volatile compound patterns in exhaled breath samples. J Infect, 2016.

19. Sahota, A.S., et al., A simple breath test for tuberculosis using ion mobility: A pilot study. Tuberculosis (Edinb), 2016. 99: p. 143-6.

20. Zhu, J., et al., Detecting bacterial lung infections: in vivo evaluation of in vitro volatile fingerprints. Journal of breath research, 2013. 7: p. 016003-016003.

21. Phillips, M., et al., Volatile biomarkers of pulmonary tuberculosis in the breath. Tuberculosis, 2007. 87(1): p. 44-52.

22. Bos, L.D., et al., Bacteria in the airways of patients with cystic fibrosis are genetically capable of producing VOCs in breath. J Breath Res, 2016. 10(4): p. 047103.

23. Shestivska, V., et al., Quantitative analysis of volatile metabolites released in vitro by bacteria of the genus Stenotrophomonas for identification of breath biomarkers of respiratory infection in cystic fibrosis. J Breath Res, 2015. 9(2): p. 027104.

24. Bean, H.D., J.-M.D. Dimandja, and J.E. Hill, Bacterial volatile discovery using solid phase microextraction and comprehensive two-dimensional gas chromatography-time-of-flight mass spectrometry. J. Chromatogr., 2012. 901: p. 41-41.

25. Boots, A.W., et al., Identification of microorganisms based on headspace analysis of volatile organic compounds by gas chromatography-mass spectrometry. Journal of breath research, 2014. 8(2): p. 027106-027106. 
26. Rees, C.A., A. Smolinska, and J.E. Hill, The volatile metabolome of Klebsiella pneumoniae in human blood. J Breath Res, 2016. 10(2): p. 027101.

27. de Heer, K., et al., eNose technology can detect and classify human pathogenic molds in vitro: a proof-of-concept study of Aspergillus fumigatus and Rhizopus oryzae. J Breath Res, 2016. 10(3): p. 036008.

28. Gordon, S.M., et al., Volatile organic compounds in exhaled air from patients with lung cancer. Clin Chem, 1985. 31(8): p. 1278-82.

29. Schallschmidt, K., et al., Comparison of volatile organic compounds from lung cancer patients and healthy controls-challenges and limitations of an observational study. J Breath Res, 2016. 10(4): p. 046007.

30. Gasparri, R., et al., Volatile signature for the early diagnosis of lung cancer. J Breath Res, 2016. 10(1): p. 016007.

31. Phillips, M., et al., Breath markers of oxidative stress in patients with unstable angina. Heart Dis, 2003. 5(2): p. 95-9.

32. Cikach Jr, F.S. and R.A. Dweik, Cardiovascular Biomarkers in Exhaled Breath. Progress in Cardiovascular Diseases, 2012. 55(1): p. 34-43.

33. Phillips, M., et al., Volatile biomarkers in the breath of women with breast cancer. J Breath Res, 2010. 4(2): p. 026003.

34. Ilan, Y., Review article: the assessment of liver function using breath tests. Alimentary Pharmacology \& Therapeutics, 2007. 26(10): p. 1293-1302.

35. Portincasa, P., et al., Liver breath tests non-invasively predict higher stages of non-alcoholic steatohepatitis. Clinical Science, 2006. 111(2): p. 135-143.

36. Park, G.J.H., et al., Non-invasive estimation of liver fibrosis in non-alcoholic fatty liver disease using the 13C-caffeine breath test. Journal of Gastroenterology and Hepatology, 2011. 26(9): p. 1411-1416.

37. Verdam, F.J., et al., Non-alcoholic steatohepatitis: A non-invasive diagnosis by analysis of exhaled breath. Journal of Hepatology, 2013. 58(3): p. 543-548.

38. Kokoszka, J., et al., Determination of inflammatory bowel disease activity by breath pentane analysis. Dis Colon Rectum, 1993. 36(6): p. 597-601.

39. Ahmed, I., et al., Investigation of faecal volatile organic metabolites as novel diagnostic biomarkers in inflammatory bowel disease. Alimentary Pharmacology \& Therapeutics, 2016. 43(5): p. 596-611.

40. Ahmed, I., et al., An Investigation of Fecal Volatile Organic Metabolites in Irritable Bowel Syndrome. Plos One, 2013. 8(3).

41. Hicks, L.C., et al., Analysis of Exhaled Breath Volatile Organic Compounds in Inflammatory Bowel Disease: A Pilot Study. Journal of Crohns \& Colitis, 2015. 9(9): p. 731-737.

42. Patel, N., et al., Metabolomic analysis of breath volatile organic compounds reveals unique breathprints in children with inflammatory bowel disease: a pilot study. Alimentary Pharmacology \& Therapeutics, 2014. 40(5): p. 498-507.

43. Pelli, M.A., et al., Breath alkanes determination in ulcerative colitis and Crohn's disease. Dis Colon Rectum, 1999. 42(1): p. 71-6.

44. Bodelier, A.G.L., et al., Volatile Organic Compounds in Exhaled Air as Novel Marker for Disease Activity in Crohn's Disease: A Metabolomic Approach. Inflammatory Bowel Diseases, 2015. 21(8): p. 1776-1785.

45. Fent, K.W., et al., Volatile Organic Compounds Off-gassing from Firefighters' Personal Protective Equipment Ensembles after Use. J Occup Environ Hyg, 2015. 12(6): p. 404-14.

46. Systemic Exposures to Volatile Organic Compounds and Factors Influencing Susceptibility to Their Effects, in Contaminated Water Supplies at Camp Lejeune: Assessing Potential Health Effects, N.A.P. (US), Editor. 2009, National Research Council (US) Committee on Contaminated Drinking Water at Camp Lejeune.

47. Lourencetti, C., et al., New method for determination of trihalomethanes in exhaled breath: Applications to swimming pool and bath environments. Analytica Chimica Acta, 2010. 662(1): p. 23-30.

48. Vereb, H., et al., The Possibilities Will Take Your Breath Away: Breath Analysis for Assessing Environmental Exposure. Environmental Science \& Technology, 2011. 45(19): p. 8167-8175.

49. Bödeker, B., et al., Biomarker validation —room air variation during human breath investigations. International Journal for Ion Mobility Spectrometry, 2010. 13(3-4): p. 177-184.

50. Joachim, D.P., A.S. Matthew, and H.R. Terence, Clinical breath analysis: discriminating between human endogenous compounds and exogenous (environmental) chemical confounders. Journal of Breath Research, 2013. 7(1): p. 017107. 
51. Das, M.K., et al., Investigation of gender-specific exhaled breath volatome in humans by GCxGCTOF-MS. Anal Chem, 2014. 86(2): p. 1229-37.

52. Stolk, R.P., et al., Universal risk factors for multifactorial diseases - LifeLines: a three-generation population-based study. European Journal of Epidemiology, 2008. 23(1): p. 67-74.

53. Tigchelaar, E.F., et al., Cohort profile: LifeLines DEEP, a prospective, general population cohort study in the northern Netherlands: study design and baseline characteristics. BMJ Open, 2015. 5(8): p. e006772.

54. Watson, J.T. and O.D. Sparkman, Introduction to mass spectrometry : instrumentation, applications and strategies for data interpretation. 4th ed. 2007, Chichester, England ; Hoboken, NJ: John Wiley \& Sons. xxiv, $818 \mathrm{p}$.

55. Smolinska, A., et al., Current breathomics--a review on data pre-processing techniques and machine learning in metabolomics breath analysis. J Breath Res, 2014. 8(2): p. 027105.

56. Engel, J., et al., Breaking with trends in pre-processing? TrAC Trends in Analytical Chemistry, 2013. 50: p. 96-106.

57. Aberg, K.M., et al., Feature detection and alignment of hyphenated chromatographic-mass spectrometric data. Extraction of pure ion chromatograms using Kalman tracking. J Chromatogr A, 2008. 1192(1): p. 139-46.

58. Engel, J., et al., Regularized MANOVA (rMANOVA) in untargeted metabolomics. Analytica Chimica Acta.

59. Besag, J. and P. Clifford, Sequential Monte-Carlo P-Values. Biometrika, 1991. 78(2): p. $301-304$.

60. Breiman, L., Random Forests. Machine Learning, 2001. 45(1): p. 5-32.

61. Berchtold, C., L. Meier, and R. Zenobi, Evaluation of extractive electrospray ionization and atmospheric pressure chemical ionization for the detection of narcotics in breath. International Journal of Mass Spectrometry, 2011. 299(2-3): p. 145-150.

62. Blake, N.J., et al., Carbonyl sulfide and carbon disulfide: Large-scale distributions over the western Pacific and emissions from Asia during TRACE-P. Journal of Geophysical ResearchAtmospheres, 2004. 109(D15).

63. Khalil, M.A.K. and R.A. Rasmussen, Global sources, lifetimes and mass balances of carbonyl sulfide (OCS) and carbon disulfide (CS2) in the earth's atmosphere. Atmospheric Environment (1967), 1984. 18(19): p. 1805-1813.

64. Verma, Y. and S.V.S. Rana, Gender differences in the metabolism of benzene, toluene and trichloroethylene in rat with special reference to certain biochemical parameters. Journal of Environmental Biology, 2003. 24(2): p. 135-140.

65. Vitali, B., et al., Impact of a synbiotic food on the gut microbial ecology and metabolic profiles. BMC Microbiol, 2010. 10: p. 4.

66. Beauchamp, J., Inhaled today, not gone tomorrow: pharmacokinetics and environmental exposure of volatiles in exhaled breath. Journal of Breath Research, 2011. 5(3). 


\section{Supplementary Material}

\section{Chemical Analysis}

\section{Sampling}

In this study, the volunteers exhaled through a mouthpiece connected to a valve of a resistance-free $3 \mathrm{~L}$ plastic bag (Tedlar bag, SKC Ltd, Dorset, UK). Any form of exercise and eating were prohibited one hour before sampling. All samples were collected in the same room to prevent the appearance of a background bias. The plastic bags were emptied by suction over a stainless-steel two-bed sorption tube, filled with carbograph 1TD/Carbopack X (Markes International, Llantrisant, Wales, UK) within $1 \mathrm{~h}$ after collection. The air-tight capped tubes were kept at room temperature until analysis. In this study $3 \mathrm{~L}$ of total breath (end-tidal and dead space air) was collected. Dead-space air comprises only a small part $(90 \mathrm{ml})$ of the total sample of exhaled air collected, and we have shown that the contribution of deadspace air to the total volume of whole breath does not lead to sensitivity issues in measuring VOCs by GC-tof-MS.

\section{Breath analysis}

Gas chromatography-(time-of-flight)-mass spectrometry (GC-tof-MS) was employed to evaluate the VOCs content of the exhaled air samples. The samples were measured in a random order and a non-targeted approach, i.e. no prior identification of the compounds was performed. The chromatographic conditions were chosen, in accordance with our previous work, to allow for the detection of different classes of volatile compounds while maximizing the separation of each compound. The volatile compounds were first released from the sorption tubes by thermal desorption using the Markes International Ultra-Unity automated thermal desorption equipment (Markes International) at $350^{\circ} \mathrm{C}$. Next, the mixture of vapor was divided into two parts, namely $90 \%$ of the samples was recollected on an identical sample tube and stored for eventual second analysis and the remaining amount, i.e. 10\% was loaded onto a cold $\left(5^{\circ} \mathrm{C}\right)$ sorption trap, from which it was released at a temperature of $300^{\circ} \mathrm{C}$ and injected to the GC-column for the GC-tof-MS analysis. The temperature of the 
GC (Thermo Fischer Scientific, Austin, USA, column: Restek RTX-5ms, $30 \mathrm{~m} \times 0.25 \mathrm{~mm}$ ID, coated with $1.0 \mu \mathrm{m} \mathrm{HP}-5$ phase) was programmed as follows: first $40^{\circ} \mathrm{C}$ for 5 minutes then it was increased by $10^{\circ} \mathrm{C}$ per minute until $270^{\circ} \mathrm{C}$. This temperature was maintained for 5 minutes. Time of flight mass spectrometry (Thermo Electron Tempus Plus, Thermo Electron Corporation, Waltham, USA) was used to detect and identify components available in the samples. Electron ionization at $70 \mathrm{eV}$ was used with $5 \mathrm{~Hz}$ scanning rate over a range of $\mathrm{m} / \mathrm{z} 35-350$ at unit mass resolution.

\section{Statistical Analysis}

Before the statistical analysis, the raw GC-tof-MS data had to be preprocessed. Uninformative parts of each chromatogram, (retention time in minutes $<1.3$ or $>23$ min) were removed. The data quality was improved by wavelets-based denoising. $\mathrm{P}$-splines were used to estimate the shape of the baseline and subtract it from the denoised chromatogram.

A list of chromatographic peaks was established for each chromatogram. These peaks were matched from sample to sample according to the Pearson correlation between the mass spectra, associated with the different chromatographic peaks. A high correlation (superior to $85 \%$ ) indicates that two peaks correspond to the same chemical compound. This information allowed us to construct a data table where each line represented a sample and each column a chemical compound. Each value in this table corresponded to the ion count measured for a specific VOC in a given sample. Finally, probabilistic quotient normalization was applied to suppress any effect related to different (overall) dilution of the samples.

As most biological data, VOCs tend to follow a log-normal distribution. To facilitate the analysis, the abundance was log-transformed. The resulting data follow a Gaussian distribution, as seen in Figure S1A, or bimodal distribution as in Figure S1B. 
A

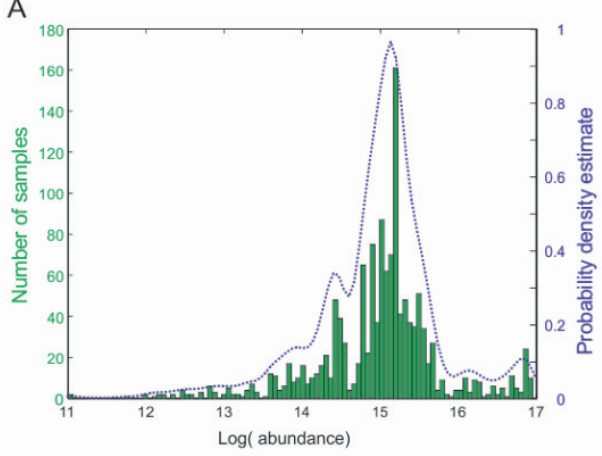

B

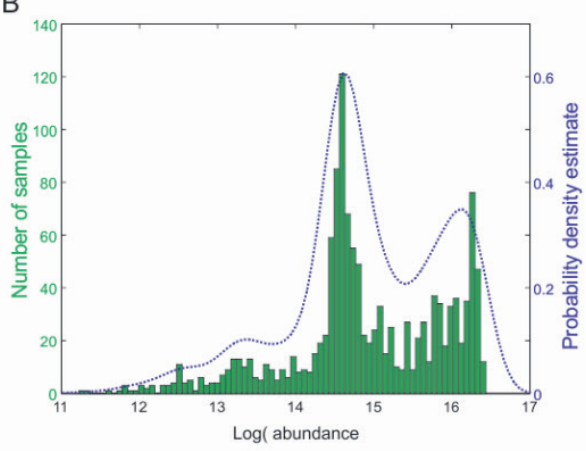

Figure S1: Distribution of the VOCs abundance after log-transformation: A) Gaussian distribution B) Bimodal distribution

The data pre-processing results in a data table reporting semi-quantitatively the concentration of 678 VOCs in 1417 samples. It is common practice to analyze a large number of samples in successive batches. Due to chromatographic drift, the VOCs profiles of different batches might be slightly different from each other. To correct for this purely technical (i.e., irrelevant) source of variance, we first detect outliers for each batch using robust PCA. Any sample with a distance (orthogonal to the model or within the model) from the batch center larger than three times the corresponding Qn estimator is flagged as outlying and excluded from any further analysis. The median for each batch is then recalculated and each batch is centred on its median position.

Breath analysis, as many metabolomics analyses, evaluates a large number of concentrations. To make the most out of this information multivariate methods are the most appropriate ones. The most established method for statistical hypotheses testing in multivariate analysis is Multivariate Analysis Of Variance (MANOVA). This approach permits to evaluate the influence of one (or multiple) factor(s) of influence, described as multiple levels e.g., gender is a factor of influence with two levels: males and females. Mathematically speaking MANOVA employs the eigenvalues of $\mathbf{J}$ as a measure of the ratio of the between group dispersion and the within-group dispersion along the direction of the eigenvectors. $\mathbf{J}$ is estimated as the ratio of $\mathbf{B}$ and $\mathbf{W}$ as shown in equation 1. B is the between covariance matrix, obtained using the cross- 
product of the $\mathbf{X}_{\mathrm{c}}$, i.e., the variance explained by a given factor $\mathrm{c}$, see equation 2 . W is the within-covariance matrix, also known as the within-group dispersion matrix, estimated from the residuals, as shown in equation 3 .

$$
\begin{aligned}
& \mathbf{J}=\mathbf{W}^{-1} \mathbf{B} \\
& \mathbf{B}=\mathbf{X}_{c}^{T} \mathbf{X}_{c}^{T} \\
& \mathbf{W}=\boldsymbol{E}^{T} \boldsymbol{E}
\end{aligned}
$$

A major drawback of MANOVA is that its inner working relies on the estimation (and inversion) of the covariance matrix $\mathbf{W}$. When the number of measured variables, here VOCs, is larger than the number of samples, here individuals, the inversion of $\mathbf{W}$ is not possible.

A regularized version of MANOVA (rMANOVA) was recently introduced in metabolomics to tackle this issue. A classical MANOVA would estimate the covariance structure from the data and would de facto put all focus on the variance and not be affected by any bias. Univariate analysis, on the other hand, is the opposite extreme, focusing on bias rather than variance. Regularization is used in many multivariate methods to obtain efficient estimators for high dimensional data. By specifying a priori, a specific covariance structure, one can select a trade-off between bias and variance. This way, correlations can be included and the model can be applied to the analysis of high dimensional metabolomics data. To estimate $p$-values we determine the distribution of the test statistic using the sequential permutation test strategy. The maximal number of permutations was set to 1000 .

The difference between means as tested above with MANOVA only answers a very specific question: are the groups different in average? This does not exclude a strong overlap between the two subpopulations tested. One may want to detect and characterize larger differences leading to a clear split between groups. In such cases discriminant models should be able to predict efficiently if a specific sample belongs to one group or another. We limit ourselves to the use of Random Forests (RF) for two reasons. First, RF is currently considered as one of the most powerful machine 
learning approaches able to deal with linear and non-linear problems, robust to outliers and handling gracefully large and/or incomplete datasets. Second, RF has been successfully applied to breath analysis in the past often surpassing other methods. Succinctly, RF builds a large collection of trees. Each tree is constructed on a randomly selected subset of the original samples and on randomly selected subsets of compounds. These trees consist of series of hierarchically organized nodes. Each node is described by a simple, logical rule based on one compound, splitting the samples into two groups. The splitting continues until the similarity of the samples within each node is the highest one or nodes contain the pre-specified minimum number of samples.

All calculations were performed using Matlab R2013a (Statistics Toolbox) and inhouse written function. The implementation of rMANOVA is available on GitHub.com (https://github.com/JasperE/regularized-MANOVA.git.

\section{Identification}

The MANOVA and RF models are based on the complete list of detected VOCs. These models can both provide lists of the most relevant VOCs. Only these relevant VOCs need to be identified. Therefore, identification is the last step of our protocol. The output of GC-tof-MS consists of two dimensions: retention time and $\mathrm{m} / \mathrm{z}$ value. Both parameters are used in the chemical identification of the VOCs. Spectrum recognition is done using the National Institute of Standards and Technology mass spectral library. A putative identification is established by searching in a reference library compounds. Potential hits are arranged in order of similarity. The final identification is performed by the manual evaluation of measured mass spectra and the reference spectra of the compounds with the highest similarity index from the "hit list", retention time information and spectrum interpretation by an experienced mass spectrometrist. From a total of 24 different chemically identified compounds, 8 were identified based on mass spectrum and retention time confirmed by independently measured model compounds, the other were tentatively identified based on mass spectrum and plausible retention times. 


\section{Selected Compounds}

The statistical analysis performed on the LL DEEP cohort lead to define Smoking, Gender, BMI and Gender as possible confounders in breath analysis. The most important for these differences are regrouped in Table 3 and together with additional references in Table S1. The relative importance of these VOCS are represented in Figure S1.

A

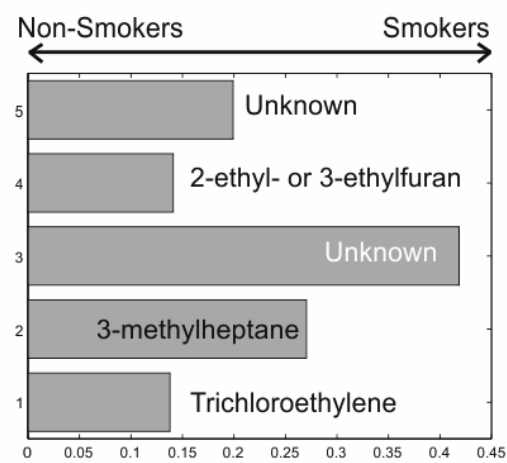

C

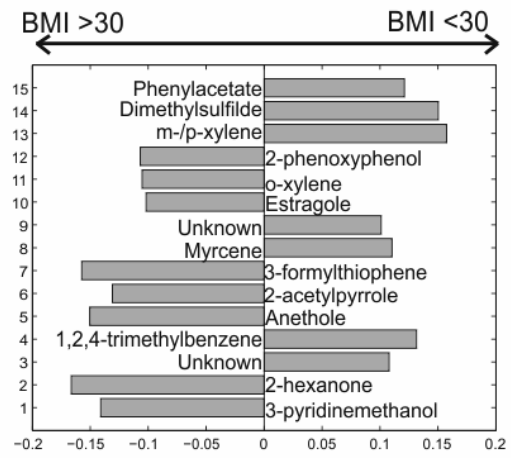

B

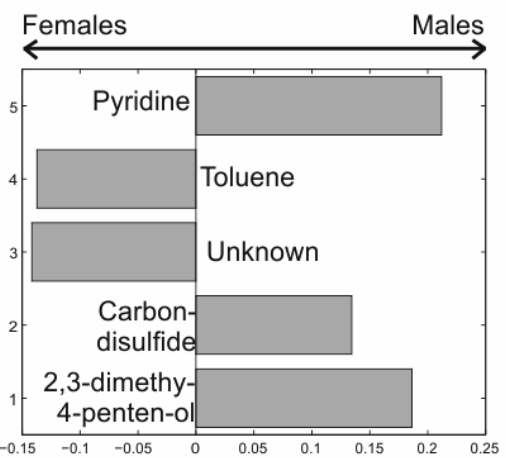

D

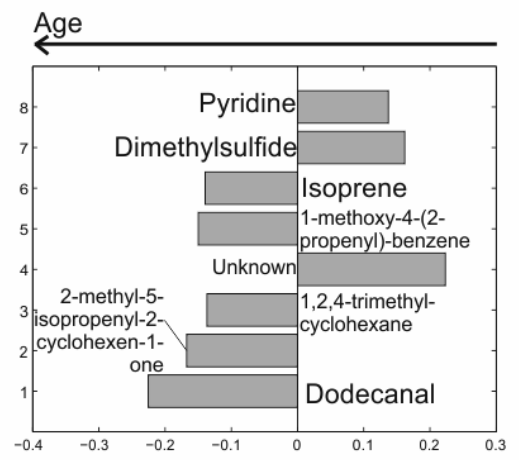

Figure S1: Relative importance of the VOCs presented in Table S1 calculated using the MANOVA model. 


\begin{tabular}{|c|c|c|c|c|c|}
\hline & RT & Identification & CAS & Notes & Refs \\
\hline \multirow{6}{*}{ 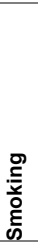 } & 7.04 & Trichloroethylene & $79-01-6$ & reported in cigarette smoke & $(18)$ \\
\hline & 8.38 & Pyridine & $110-86-1$ & & $(19,20)$ \\
\hline & 9.07 & 3-methylheptane & $589-81-1$ & Related to inflammation & $(21)$ \\
\hline & 11.71 & 2-ethyl- or 3-ethylfuran & $3208-16-0$ or & Furans reported in cigarette smoke & $(18)$ \\
\hline & & & $67363-95-5$ & & \\
\hline & 14.80 & Unknown & & & \\
\hline \multirow{5}{*}{$\frac{\grave{D}}{\frac{1}{0}}$} & 2.98 & Carbon disulfide (CS2) & $75-15-0$ & Exposure more common in males & $(22)$ \\
\hline & 3.83 & Unknown & & & \\
\hline & 8.38 & Pyridine & $110-86-1$ & Roasted coffee & $(23)$ \\
\hline & 9.07 & Toluene & $108-88-3$ & $\begin{array}{l}\text { Exposure, gender difference in } \\
\text { metabolism }\end{array}$ & $(24,25)$ \\
\hline & 15.29 & 2,3-dimethyl-4-penten-ol & $19781-52-3$ & & \\
\hline \multirow[b]{16}{*}{$\sum_{m}$} & 2.61 & Dimethylsulfide & $75-18-3$ & methionine metabolism & $(26)$ \\
\hline & 9.82 & 2-hexanone & $591-78-6$ & $\begin{array}{l}\text { Endogenous ketone, reported in sepsis in } \\
\text { rats }\end{array}$ & $(27,28)$ \\
\hline & 11.46 & m-/p-xylene & $108-38-3 / \quad 106-$ & Exposure & $(29)$ \\
\hline & & & $42-3$ & & \\
\hline & 12.10 & o-xylene & $95-47-6$ & Exposure & $(29)$ \\
\hline & 13.52 & 1,2,4-trimethylbenzene & $95-63-6$ & Exposure & \\
\hline & 13.87 & $\begin{array}{l}\text { 7-methyl-3-methylene-1,6- } \\
\text { octadiene }\end{array}$ & $123-35-3$ & $\begin{array}{l}\text { (Myrcene) Flavoring agent, reported in } \\
\text { breath }\end{array}$ & $(30,31)$ \\
\hline & 14.00 & 3-formylthiophene & $498-62-4$ & & \\
\hline & 14.63 & 2-acetylpyrrole & $1072-83-9$ & $\begin{array}{l}\text { Used in flavors for tobacco and roasted } \\
\text { foods }\end{array}$ & $(32)$ \\
\hline & 14.65 & 3-pyridinemethanol & $100-55-0$ & & \\
\hline & 15.29 & Phenyl acetate & $122-79-2$ & $\begin{array}{l}\text { Downstream product of aspartame and } \\
\text { phenylalanine }\end{array}$ & $(33,34)$ \\
\hline & 16.48 & Unknown & & & \\
\hline & 17.44 & Unknown & & & \\
\hline & 18.94 & 1-methoxy-4-(1-propenyl)-benzene & $104-46-1$ & (Anethole) flavoring agent & (35) \\
\hline & 19.34 & 1-methoxy-4-(2-propenyl)-benzene & $140-67-0$ & (Estragole) flavoring agent & $(35)$ \\
\hline & 22.73 & 2-phenoxyphenol & $2417-10-9$ & & \\
\hline \multirow[b]{8}{*}{ 号 } & 2.58 & Isoprene & $78-79-5$ & Related to cholesterol, age and gender & $(36,37)$ \\
\hline & 2.61 & Dimethylsulfide & $75-18-3$ & methionine metabolism - & $(26)$ \\
\hline & 8.38 & Pyridine & $110-86-1$ & & \\
\hline & 11.63 & 1,2,4-trimethylcyclohexane & $2234-75-5$ & & \\
\hline & 18.38 & Unknown & & & \\
\hline & 18.68 & $\begin{array}{l}\text { 2-methyl-5-isopropenyl-2- } \\
\text { cyclohexen-1-one }\end{array}$ & $99-49-0$ & $\begin{array}{l}\text { (Carvone) terpenoids naturally found in } \\
\text { oils from seeds of caraway (Carum carvi), } \\
\text { spearmint (Mentha spicata), }\end{array}$ & $(35,38)$ \\
\hline & 19.34 & 1-methoxy-4-(2-propenyl)-benzene & $140-67-0$ & (Estragole) flavoring agent & (35) \\
\hline & 21.66 & Dodecanal & $112-54-9$ & $\begin{array}{l}\text { (Lauraldehyde) fragrance, seen on the } \\
\text { skin, related to gluten free }\end{array}$ & $(39,40)$ \\
\hline
\end{tabular}

Table S1: Interpretation and bibliographic investigation of the selected VOCs driving the differences between the groups stratified according to smoking behavior, gender, BMI and age. 


\begin{tabular}{|c|c|c|c|c|c|}
\hline Nr. & $\begin{array}{l}\text { Retention time } \\
\text { (min) }\end{array}$ & Identification & $\begin{array}{l}\text { Relative } \\
\text { abundance } \\
\text { (mean) }\end{array}$ & $\begin{array}{l}\text { Relative } \\
\text { abundance } \\
\text { (STD) }\end{array}$ & $\begin{array}{l}\text { Frequency } \\
(\%) \text { in } n=1417\end{array}$ \\
\hline 1 & 2.58 & Isoprene & $1,17 E+08$ & $9,19 E+07$ & 93,6 \\
\hline 2 & 2.61 & Dimethylsulfide & $1,85 \mathrm{E}+07$ & $2,87 E+06$ & 48,5 \\
\hline 3 & 2.98 & Carbon disulfide (CS2) & $4,15 E+06$ & $1,56 \mathrm{E}+06$ & 41,0 \\
\hline 4 & 3.83 & Unknown & $3,01 E+06$ & $1,38 \mathrm{E}+06$ & 50,7 \\
\hline 5 & 7.04 & Trichloroethylene & $5,88 \mathrm{E}+05$ & $9,89 \mathrm{E}+05$ & 21,6 \\
\hline 6 & 8.38 & Pyridine & $7,51 \mathrm{E}+07$ & $5,46 \mathrm{E}+07$ & 65,9 \\
\hline 7 & 9.07 & 3-methylheptane & $2,49 E+04$ & $3,59 \mathrm{E}+03$ & 21,9 \\
\hline 8 & 9.07 & Toluene & $6,71 \mathrm{E}+06$ & $6,18 \mathrm{E}+06$ & 99,9 \\
\hline 9 & 9.82 & 2-hexanone & $1,90 \mathrm{E}+06$ & $2,17 \mathrm{E}+06$ & 86,9 \\
\hline 10 & 11.46 & m-/p-xylene & $1,37 E+07$ & $2,85 E+07$ & 34,2 \\
\hline 11 & 11.63 & 1,2,4-trimethylcyclohexane & $1,38 \mathrm{E}+07$ & $2,87 \mathrm{E}+07$ & 32,2 \\
\hline 12 & 11.71 & 2-ethyl- or 3-ethylfuran & $3,94 \mathrm{E}+07$ & $1,73 \mathrm{E}+07$ & 20,1 \\
\hline 13 & 12.10 & o-xylene & $1,53 \mathrm{E}+07$ & $5,82 \mathrm{E}+06$ & 26,3 \\
\hline 14 & 13.52 & 1,2,4-trimethylbenzene & $6,58 \mathrm{E}+06$ & $4,61 \mathrm{E}+06$ & 24,3 \\
\hline 15 & 13.87 & 7-methyl-3-methylene-1,6-octadiene & $6,43 \mathrm{E}+07$ & $3,77 \mathrm{E}+07$ & 27,0 \\
\hline 16 & 14.00 & 3-formylthiophene & $1,02 \mathrm{E}+07$ & $5,43 E+06$ & 27,2 \\
\hline 17 & 14.63 & 2-acetylpyrrole & $5,52 \mathrm{E}+06$ & $2,82 E+06$ & 21,1 \\
\hline 18 & 14.65 & 3-pyridinemethanol & $2,99 E+06$ & $2,36 \mathrm{E}+06$ & 25,1 \\
\hline 19 & 14.80 & Unknown & $1,50 \mathrm{E}+07$ & $1,63 E+07$ & 22,4 \\
\hline 20 & 15.29 & 2,3-dimethyl-4-penten-ol & $3,78 \mathrm{E}+06$ & $2,96 \mathrm{E}+06$ & 33,8 \\
\hline 21 & 15.29 & Phenyl acetate & $5,23 E+06$ & $4,74 \mathrm{E}+06$ & 46,8 \\
\hline 22 & 16.48 & Unknown & $3,18 \mathrm{E}+07$ & $1,82 \mathrm{E}+07$ & 29,8 \\
\hline 23 & 17.44 & Unknown & $9,57 \mathrm{E}+06$ & $8,99 E+06$ & 33,3 \\
\hline 24 & 18.38 & Unknown & $1,50 \mathrm{E}+07$ & $1,63 \mathrm{E}+07$ & 22,4 \\
\hline 25 & 18.68 & 2-methyl-5-isopropenyl-2-cyclohexen-1-one & $8,83 \mathrm{E}+06$ & $4,38 \mathrm{E}+06$ & 28,7 \\
\hline 26 & 18.94 & 1-methoxy-4-(1-propenyl)-benzene & $5,89 \mathrm{E}+06$ & $1,16 \mathrm{E}+06$ & 23,2 \\
\hline 27 & 19.34 & 1-methoxy-4-(2-propenyl)-benzene & $6,15 \mathrm{E}+06$ & $2,70 \mathrm{E}+06$ & 32,3 \\
\hline 28 & 21.66 & Dodecanal & $1,90 \mathrm{E}+06$ & $2,17 \mathrm{E}+06$ & 94,6 \\
\hline 29 & 22.73 & 2-phenoxyphenol & $3,19 \mathrm{E}+06$ & $3,55 \mathrm{E}+06$ & 38,6 \\
\hline
\end{tabular}

Table S2: The relative abundance of 29 VOCs being significantly affected by one of the factors indicated in Table S1. The relative abundance is represented as mean and standard deviation (STD). The information on the frequency of appearance of the compound is included as well. 


\section{Abstract}

In the present longitudinal study, we followed volatile organic compounds (VOCs) excreted in exhaled breath of twenty healthy individuals over time, while adhering to a gluten-free diet for four weeks prior to adherence to a normal diet. We used Gas Chromatography coupled with Mass Spectrometry (TD-GC-tof-MS) in combination with chemometric analysis to detect an array of VOCs in exhaled breath. Multivariate analysis was applied to extract the maximal information from the obtained data. Dietary intake was assessed to verify adherence to the diet and to get insight into macronutrient intake during the intervention period. A set of 12 volatile compounds distinguished the samples obtained during the gluten-free diet from those obtained during a normal diet. Seven compounds could be chemically identified (2-butanol, octane, 2-propyl-1pentanol, nonanal, dihydro-4-methyl-2(3H)-furanone, nonanoic acid and dodecanal) and speculated on a possible origin. Our findings suggest that a gluten-free dietary period had a reversible impact on participants' excreted metabolites visible in their breath. Several explanations are proposed of influencing metabolic status through dietary interventions. Although the exact origin of the discriminating compounds is not yet known, the main goal of this paper was to share a new potential use of exhaled air analysis and might become a useful tool in fields of nutrition and metabolism. 


\section{Introduction}

Analysis of exhaled breath in health-related issues has become a promising subject due to its non- invasive nature and a growing number of applications [1]. A single breath sample contains 300-500 volatile organic compounds (VOCs) occurring in $\mathrm{nmol} / / \mathrm{-pmol} / \mathrm{l}$ concentrations and can be detected with several chemical analytical approaches including gas chromatographic and mass spectrometric based methods [2]. Over the last few decades, a tremendous progress in the methodology development (both instrumental and computational) improved the field of breath analysis and has the promise of a practical, reliable, reproducible, and clinically relevant source of information about human health [1]. Due to intra- and inter individual differences the total number of VOCs in a series of samples may be as high as 3000-5000. VOCs analysis is routinely used in the assessment of environmental contamination [3], fragrance and flavor industries [4] and forensic science [5]. In medical research VOCs are related to several disease conditions including chronic inflammatory lung diseases [6,7], non-alcoholic steatohepatitis (NASH) [8] and gastrointestinal diseases [9, 10]. Important issues that still need to be solved are related to standardization of methodologies, sampling of breath, and insights in confounding factors including the influence of diet on profiles of excreted VOCs. Although diet often is suggested to be one of the exogenous factors influencing composition of exhaled breath [11], research into this is lacking.

In the present study we followed excreted VOCs in individuals over time while adhering to a gluten-free diet for four weeks prior to adherence to a normal diet. A gluten-free diet is based on the exclusion of protein complex consisting of gluteins and prolamins present in several cereals, like wheat, barley, and rye [12]. Wheat supplies about $20 \%$ of the total food calories for humans and is a key source of plant proteins [13]. Unfortunately, it is also a major cause of food-induced health issues. The most recognized enteropathy caused by an aberrant immune response to gluten protein is celiac disease, for which a gluten-free diet is the only known treatment [14, 15]. A gluten free diet was previously reported to modify the composition and immune properties of the gut microbiota in both healthy subjects [16] and celiac disease patients [1]. A change in a host microbiota would be expected to be associated with 
a change of volatile metabolites produced, released in the lungs and then detected in exhaled breath. In this study we aimed to identify specific metabolites after a gluten-free diet by performing a longitudinal study of 20 healthy volunteers following a gluten-free diet for 4 weeks and returning to normal diet for 9 weeks.

\section{Materials and methods}

\section{Study participants}

Twenty participants without any known food intolerance were involved in this study (female: 12, male: 9, 16-61years (average 36 years), BMI 18.9-35.3 (average 24)). Except one, none of the participants was treated with antibiotics for at least one year prior to the sample collection. Participants were instructed to exclude gluten containing products from a daily diet by replacing them with certified gluten-free products for a period of 4 weeks. Afterwards they were asked to return to their habitual diet and lifestyle. All participants adhered voluntary to gluten containing product free diet for 4 weeks.

The authors received written consents from all participants and followed the sampling protocol of the LL Deep study approved by the UMCG ethical committee.

\section{Dietary assessment and analysis}

Dietary intake was assessed to verify adherence to the gluten-free diet and to gain insight into differences in macronutrient intake in healthy volunteers on gluten-free diet compared to their normal diet. All participants received information about glutencontaining products from a dietician and were instructed how to keep a 3-day food record including two weekdays and one day in the weekend. The food records included data regarding the moment of the day food was eaten and the type and quantity of food consumed. Participants were instructed how to estimate serving sizes and to record recipes. After returning the food records, participants were contacted by the dietician to clarify uncertainties within the food record. Intake of macronutrients was calculated using the website www.voedingsdagboek.nl. This website incorporates the Dutch Food Composition Database [17]. 
We used SPSS software version 20 (IBM SPSS Statistics release 20.0.0.1) for statistical analysis of the dietary intake data. Mean and standard deviation of daily intake was calculated for energy (kcal), protein $(\mathrm{g})$, carbohydrate $(\mathrm{g})$, and fat $(\mathrm{g})$. The paired t-test was used to compare group means during gluten-free diet versus normal diet.

\section{Experimental design}

Collection of breath samples was spread over a 13-week period, as indicated in Figure 1. First samples were taken directly before the start of the gluten-free diet period (T0). For 4 weeks, the participants were on a gluten-free diet by replacing normal gluten-containing food with certified gluten free products. Breath samples were collected at the end of week 1(T1), 2(T2), 3(T3) and 4(T4). Next, participants were asked to return to their usual diet. Follow-up samples were collected at the end of week 13(T13) (see figure 1).

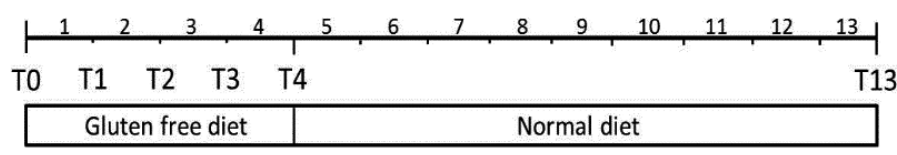

Figure 1. Scheme representing the experimental design of the collection of breath samples over the 13 weeks period. The time points for sampling collection are indicated as TO-T13.

\section{Sampling}

For exhaled breath collection participants were asked to inflate 3 Liter bags (Tedlar Bag, SKC Ltd, Dorset, UK). Within 2 hours after collection the bag contents were transferred to carbon-filled stainless steel sorption tubes where the VOC's were trapped (Markes International, Llantrisant Business Park, UK). Tubes were stored at room temperature until analysis. 


\section{Analysis}

Samples were analyzed by thermal desorption-gas chromatography combined with time-of-flight mass spectrometry (GC-tof-MS) as previously described [18]. The VOCs were released from the sorption tubes under a flow of helium using automated thermal desorption (Markes International Ultra-Unity). The gaseous mixture is then split: $90 \%$ of the sample is recollected on an identical tube and $10 \%$ of the sample is transferred to an electrically cooled sorbent trap $\left(5^{\circ} \mathrm{C}\right)$ and from there injected into the gas chromatograph (Trace GC, Thermo Fischer Scientific, Austin, USA, column: Restek RTX-5ms, 30mx0.25mm ID, coated with $1.0 \mu \mathrm{m}$ HP-5 phase. Carrier gas helium at a flow rate of $1.5 \mathrm{~mL} / \mathrm{min}$. GC temperature was programmed as follows: $40^{\circ} \mathrm{C} / 5 \mathrm{~min}$., then increased by $10^{\circ} \mathrm{C} / \mathrm{min}$. until $270^{\circ} \mathrm{C}$ at which temperature was maintained for $5 \mathrm{~min}$.). After the separation by GC the components were analyzed by time-of-flight mass spectrometry (Tempus Plus, Thermo Fischer Scientific). The MS was set at as follows: ionizing energy $=70 \mathrm{eV}$, mass range 35-350 AMU, scanning rate five times per second.

\section{Data handling}

\section{Data preprocessing}

Before the actual multivariate analysis, important issues such as variations in baseline and peak shifts must be treated. The raw GC-tof-MS spectra require several consecutive steps including denoising, baseline removal, alignment, and scaling. Usually, the beginning and the end of each chromatogram (retention time in minutes either $<1.3$ or $>23 \mathrm{~min}$ ) have to be removed beforehand because of noisy mass spectra and column bleeding. The first step consists of noise removal and baseline correction. Denoising was performed by means of wavelets [19], while baseline was corrected using P-splines [20]. This approach uses asymmetric least-squares smoothing, by penalizing regions with signal in the chromatogram, to find the data points corresponding to the baseline. Baseline is adjusted in such a way that ideally only contributions from eluting compounds are enclosed in the signal. The drift in the peaks was eliminated by piecewise alignment method correlation optimized warping (COW), which can handle non-systematic global shifts [21]. Finally, the 
chromatograms for each sample were merged by combining the corresponding compounds based on retention time and similarities in mass spectra. To make the chromatograms comparable the final step of preprocessing involved normalization to total area and the data were scaled to unite variance. Unite variance scaling takes into account the differences in orders of magnitude between measured compounds. After this step each compound has the same variance so that all compounds become equally important in the analysis.

\section{Multivariate data analysis}

Multivariate statistical methods provide an expert means of analyzing and maximizing information recovery from complex GC-tof-MS data. GC-tof-MS data are numerically complex and contain thousands of irrelevant and/or redundant information (i.e., variables). Moreover, many variables increase collinearity in the data. Variable selection allows picking the subset of best predictors (variables). Variables differently profiled across the groups of interest were selected based on ANalysis of VAriance-Principal Component Analysis (ANOVA-PCA), which is a combination of two methods, ANOVA and PCA [22]. This technique together with ANOVA and multivariate-ANOVA (MANOVA) belong to the family of ANOVA methods. ANOVA-PCA in comparison to standard ANOVA and MANOVA is applicable to large data, i.e., data containing more variables than samples. The main aim of ANOVA-PCA is to split the total variance in the data into the variance of the effect of interest (i.e., differentiate groups) and other irrelevant sources of variance (e.g., time effect). The significant subset of VOCs is selected using multivariate t-test (Hotelling test). The significant VOCs are the one whose concentrations are affected by the diet and thus are expected to have p-value smaller or equal 0.05 . Note that this test is a multivariate test; therefore, it is only performed once, implying that multitesting correction is not required. The detailed information about the statistical background of ANOVA-PCA can be found elsewhere [23, 24].

The relevant subset of VOCs was next used in the PCA analysis [25]. PCA extracts and displays the systematic variation in the data. The PCA model provides an overview, of all samples in the data. In addition, grouping, trends and outliers can be 
found. PCA converts the multidimensional data space into a low-dimensional model plane. This technique expresses most of the variance within a data set using a smaller number of factors, so called principal components (PCs). PCA decomposes the original data set into two matrices known as scores and loadings. Scores are the coordinates for the samples. In a scores plot, each point represents a single spectrum. It provides a summary of all spectra and shows how they are related to each other. Hence, the points that are close to each other have similar profile, while objects that lie far away are characterized by different properties. In the loading plot the relation among measured VOCs can be visualized. The angle between compounds reflects the correlation between them. The correlation is high if the angle is small. The angle close to $180^{\circ}$ indicates an anti-correlation. The two compounds are not correlated if the angle is close to $90^{\circ}$. An important feature is that the directions in the score plot correspond to directions in the loading plot. Therefore, it is possible to combine loadings and scores into so called biplot. In the biplot every point corresponds to a sample and every arrow (line) to a compound. A very useful feature of the biplot is the possibility of defining the change of compounds relative concentration due to different diet. The concentration is elevated if the sign of the new coordinate (i.e., PCs) for samples and compound is the same (i.e., either both positive or both negative). If the sign of the new coordinate for samples and compound is opposite (i.e., positive, and negative) the concentration is down regulated in these samples.

\section{Results}

\section{Multivariate analysis}

In total, 107 breath samples were analyzed, and GC-tof-MS spectra were obtained. Each chromatogram consists of 3651 variables. The data set contains a lot of zeros, since not every compound is present in each breath sample. In order to focus on the most informative part of the data (non-zeros), the following procedure was applied, which will be referred here as the ' $20 \%$ rule'. A variable is kept if it is nonzero for at least $20 \%$ of all samples in at least one of the experimental groups. After the application of the $20 \%$ rule, the data set contains 527 variables. One chromatogram was removed from the analysis because of low signal to noise ratio. The remaining 
106 chromatograms were used in the analysis. An example of a preprocessed chromatogram is shown in figure 2.

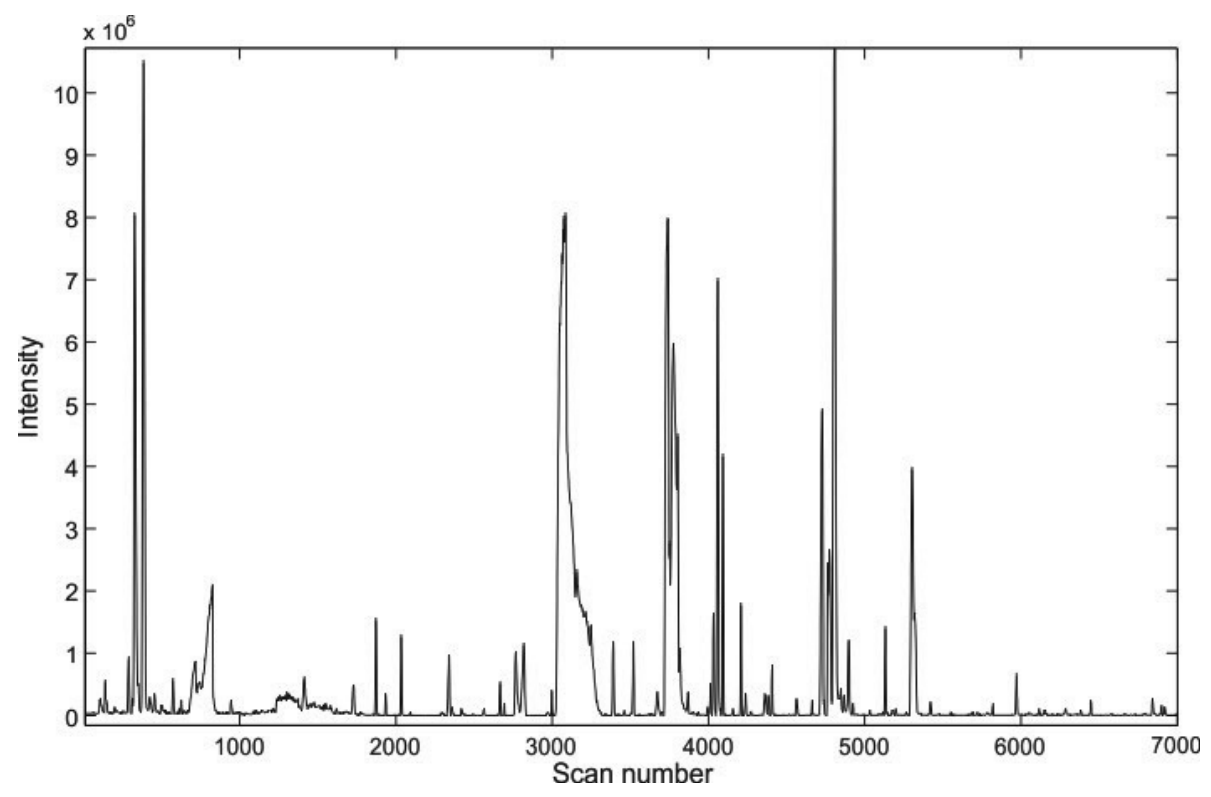

Figure 2. An example of a preprocessed chromatogram obtained with GC-tof-MS.

Our multivariate analysis involved two parts. In the first one ANOVA-PCA is used to select the significant compounds affected by the diet. The second step consisted of PCA model built on the data containing only the relevant compounds picked up by ANOVA-PCA. ANOVA-PCA was performed on a subset of the data including the time points: T3, T4 (period of gluten-free diet) and T0, T13 (period of normal diet). The reasoning behind the selection of this subset is twofold. Firstly, for proper and reliable ANOVA it is necessary to include all time points per individual because time is one of the main effects. Therefore, volunteers were excluded in this part of analysis when a point T0, T3, T4 or T13 was missing. Secondly, the preliminary analysis (based on PCA) showed no differences in VOCs profile between the period of normal diet (time points T0, T13) and gluten-free diet (time points T1 and T2). However, to use the available data fully the remaining time points T1, T2 were later projected into PCA space. It has to be pointed out that for time points T0, T3, T4 and T13 only 14 
participants delivered their breath samples. For the remaining volunteers, the breath samples were missing due to different circumstances (e.g., participant absence during collection or poor GC-tof-MS spectrum).

The ANOVA-PCA analysis allowed for selection of 12 compounds with p-values smaller than 0.05. As mentioned in Materials and Methods section it is not necessary to use multitesting correction when multivariate t-test is applied. Multivariate t-test selects a subset of significant VOCs, therefore the number of statistically relevant compounds can be lower than $\sim 25$ (i.e., $5 \%$ of 527). In the next step the chemical identification of these 12 VOCs was done by means of spectrum recognition using the NIST library in combination with spectrum interpretation by an experienced Mass-Spectrometrist and identification based on retention times of components. From a set of 12 compounds 7 were chemically identified, while rest remained unknown. Some compounds could not be identified due to insufficient mass spectrum, overlap in the retention time or absence of mass spectrum in the library.

To visualize the results, 12 selected compounds were re-analyzed in a separate PCA analysis. The corresponding biplot is shown in figure 3(a). Each triangle and star correspond to a chromatogram, each line to a compound. As can be seen the separation between normal diet (samples indicated as stars) and gluten-free diet (samples indicated as triangular) is reached along two first principal components (explaining almost $50 \%$ of total variance). However, some of the gluten-free diet samples, on the left of the biplot, seem to be more similar to a normal diet, since they overlap with them. This suggests that the response to gluten-free diet was heterogeneous between the volunteers.

Interestingly, the volunteers in gluten-free diet group are characterized by higher variations in comparison to the same volunteers group following the normal diet. This is clearly visible in figure $3(\mathrm{a})$, where the spread of points in gluten-free diet is larger than in the normal diet group. This is probably related to the previous point about the heterogeneous response to gluten-free diet leading to larger variations in exhaled volatile products. Additionally, in figure $3(a)$ the changes in relative compound 
concentrations in the breath samples are indicated for each compound by a dashed line. The relative concentrations of octane and 2-butanol point towards the normal diet group, indicating a higher level in normal diet (the sign of PC1 for samples (stars) and compounds (lines) is the same, i.e., negative) and reduced levels in the glutenfree diet group (the sign of PC1 for samples (triangular) and compounds (lines) is opposite, i.e., positive for samples and negative for compounds). On the contrary nonanal and dodecanal levels are elevated in gluten-free diet, because PC1 has the same sign (here positive) for samples (triangular) and compounds (lines).

Similarly, to traditional boxplot, biplot allows representing not only the changes in relative compound concentrations but also the relation between the compounds (i.e., the correlation between them). Nonanal and dodecanal are highly correlated as can be seen from a very small angle of the two corresponding lines. This is corroborated by boxplot in figure 3(b), where both compounds follow the same trend, i.e., their amounts are elevated in gluten-free diet group. The reverse observation can be made concerning the relation between octane and dodecanal. These two compounds are negatively correlated, as can be seen by the large angle formed by two corresponding lines in the biplot. This is reaffirmed by boxplot shown in figure $3(b)$, where the level of octane is reduced in gluten-free diet group. Thus, the concentration of octane changes contrariwise to levels of dodecanal and nonanal.
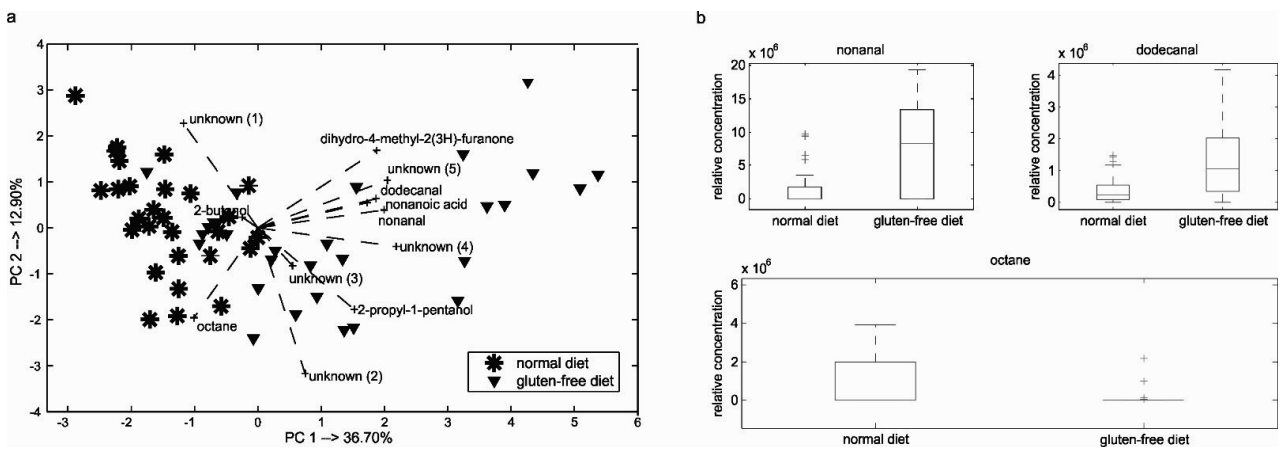

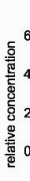

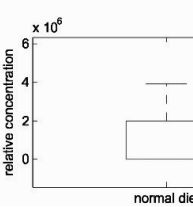

octane

Figure 3. (a) Biplot of PCA analysis performed on 12 significant VOCs excreted in breath samples obtained at the end of week 3 and 4 of gluten-free diet (times points T3, T4) and those for a normal diet (time point TO and T13). (b) The boxplots of nonanal, dodecanal and octane shown for samples obtained at the end of week 3 and 4 of gluten-free diet and those for normal diet. 
Table 1 shows the identified and unidentified VOCs and their relative concentrations change in breath samples obtained during gluten-free-diet period. Up or down regulation of VOC concentration is indicated as $(+)$ or (-), respectively, with reference to normal diet.

\begin{tabular}{|c|c|}
\hline VOCs & Group \\
\hline & gluten-free-diet \\
\hline 2-butanol & $(-)$ \\
\hline Octane & $(-)$ \\
\hline 2-Propyl-1-pentanol & $(+)$ \\
\hline Nonanal & $(+)$ \\
\hline Dihydro-4-methyl-2(3H)-furanone & $(+)$ \\
\hline Nonanoic acid & $(+)$ \\
\hline Dodecanal & $(+)$ \\
\hline Unknown (1) & $(-)$ \\
\hline Unknown (2) & $(+)$ \\
\hline Unknown (3) & $(+)$ \\
\hline Unknown (4) & $(+)$ \\
\hline Unknown (5) & $(+)$ \\
\hline
\end{tabular}

Table 1. A list of 12 VOCs which concentrations were significantly affected by gluten-free diet. (-) indicates decrease in VOC concentration, while (+) increase in gluten-free diet with reference to normal diet.

Breath samples were obtained from the individual volunteers at the consecutive time points and therefore it is possible to see the individual trajectories along the experiment (i.e., T0, T3, T4 and T13). In figure 4, the PCA score plot defined by the first two PCs is displayed with samples symbolized accordingly to T0, T3, T4 and T13 time points. The trajectories of 4 participants are included in the figure as arrows following the time points. It can be seen that three participants (purple, pink and red arrows) have very long trajectories in comparison to participant 4 (green arrow). This might indicate that individuals had diverse strength in responses to gluten-free diet. 
More interestingly all participants after the gluten-free intervention came back to their original position.
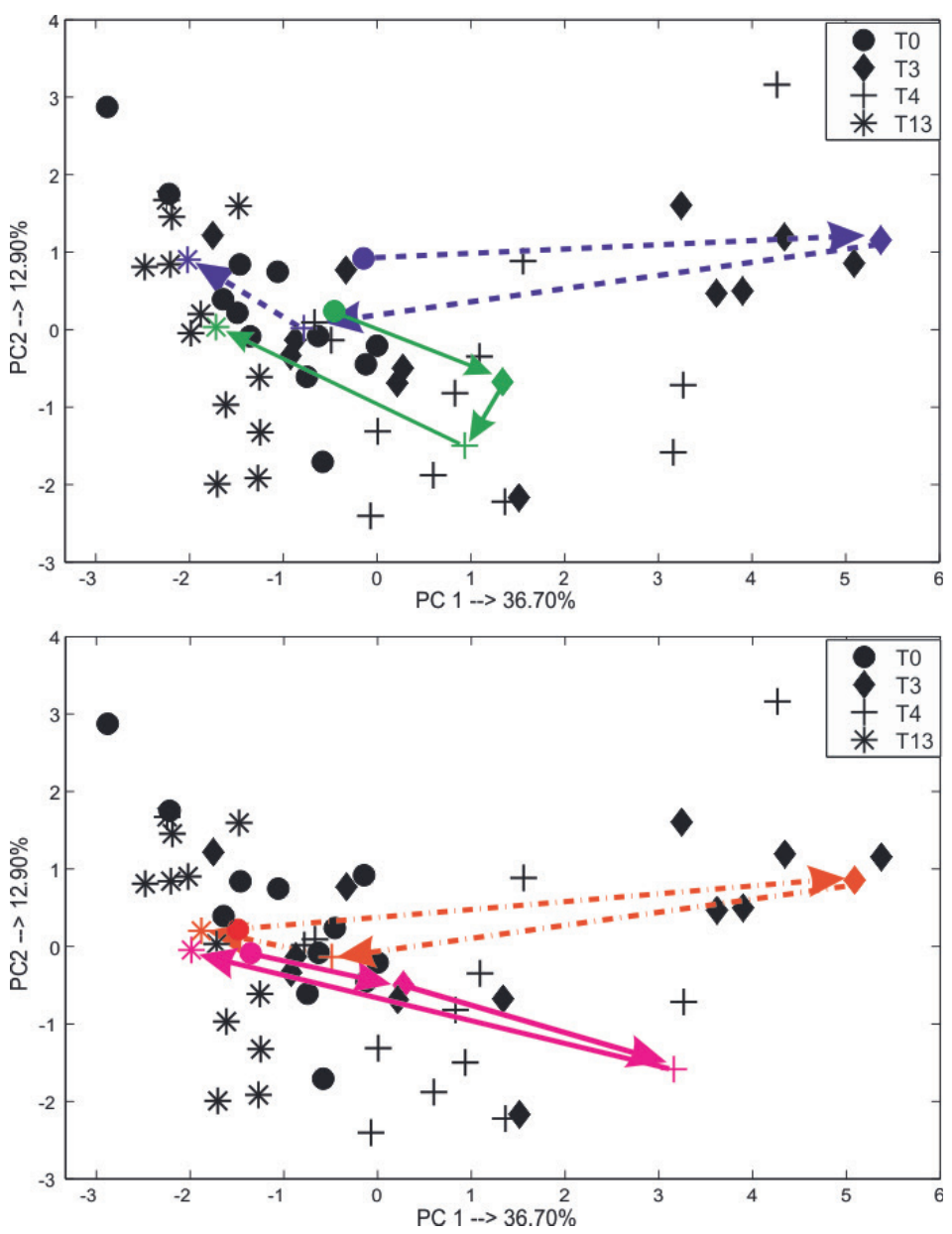

Figure 4. PCA score plot with indicated trajectories for 4 individuals. Samples are marked accordingly to 3 and 4 weeks of gluten-free diet period (T3 and T4) and normal diet (T0 and T13). Each trajectory is plotted as an arrow of different color (purple: participant 1; green: participant 2; pink: participant 3; red: participant 4).

To use the available data exhaustively, the samples belonging to $T 1, T 2$, are projected into the same PCA model performed on the subset of the data as shown earlier in figure 3(a). The coordinates, i.e., scores along PC1 and PC2 are obtained for each sample belonging to T1 and T2 time points. Subsequently, the similarity of 
these samples can be compared with the ones used in the previous analysis of the subset (T0, T3, T4 and T13). For that purpose, the mean values as well as standard deviations for PC1 and PC2 were calculated for samples belonging to T1 and T2 time points. The groups' means (marked as a circle) and standard deviations (marked as a dashed line for T1 time point and solid line for T2) are plotted in figure 5. It can be observed that the mean value of gluten free diet time points (T1 and T2) progressively moves right, away from the normal diet. Interestingly the standard deviation of $\mathrm{T} 1$ is larger than that of $\mathrm{T} 2$, indicating that the variation in the $\mathrm{T} 1$ samples is more similar to normal diet. It is noticeable from Figure 5 that the standard deviations for gluten free-diet objects ( $\mathrm{T} 1$ and $\mathrm{T} 2$ ) are very large. This corroborates our findings about large variations in gluten-free diet samples.

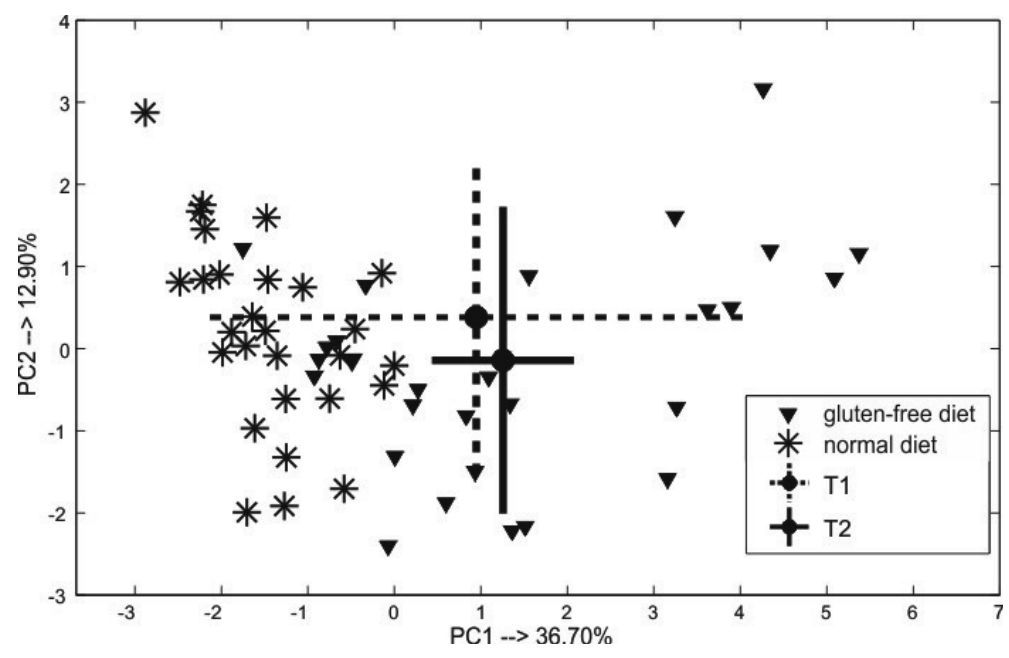

Figure 5. PCA score plot obtained from the analysis of normal diet samples (stars, T0 and T13) together with samples after 3 and 4 weeks of gluten-free diet period (triangles, T3 and T4). The samples of glutenfree diet period T1 and T2 are projected in the same PCA plane and are represented by their groups' mean and standard deviations (mean \pm std). Each mean is marked as a circle, while standard deviation as a dashed line for time points T1 and a solid line for period T2. For each time point all available samples were used. Along time the VOCs fingerprint move progressively away from the normal diet toward the gluten-free diet.

To summarize, the multivariate analysis of the breath-o-grams pinpointed a set of 12 compounds that were differentially excreted in breath of human volunteers after a period gluten-free diet. Focusing on these 12 compounds we were able to 
distinguish between gluten-free diet and normal diet within the same group of volunteers. Moreover, it is possible to track a progressive trend in the VOCs profiles in breath along the four weeks of the gluten-free diet. The large spread of variations in the exhaled VOCs within the gluten-free diet groups reflects a large heterogeneity in the response of the volunteers. After finishing the gluten-free intervention period the volunteers VOCs profiles change once more, becoming similar to the original profile seen for T0 time point of normal diet.

\section{Food intake}

15 out of 20 volunteers completed a 3-day food record (including two weekdays and one day in the weekend) for the gluten-free diet period and 14 participants completed the food record for the normal diet period. Consequently 14 participants were included in the analysis of food intake. Mean daily intake of energy (kcal), protein (gram), carbohydrate (gram), and fat (gram) per participant was calculated. The macronutrient and energy intakes data were analyzed by paired t-test. In table 2 the mean values and standard deviation and p-value obtained from paired t-test for diverse macronutrient intake are shown.

\begin{tabular}{lccccc}
\hline & \multicolumn{2}{c}{ Gluten-free diet } & Normal diet & t-test \\
\hline Mean & std & Mean & std & p-value \\
Energy (kcal) & 1709.5 & 344.0 & 1811.5 & 433.9 & 0.243 \\
\hline Protein (gram) & 73.1 & 18.4 & 78.1 & 18.2 & 0.401 \\
\hline Carbohydrates (gram) & 211.1 & 50.3 & 199.9 & 63.2 & 0.275 \\
\hline Fat (gram) & 63.7 & 18.1 & 72.5 & 24.3 & 0.109 \\
\hline
\end{tabular}

Table 2. Mean and standard deviation (std) of energy, protein, carbohydrates, and fat intake during gluten-free diet and normal diet. The last column shows the $p$-value obtained from paired t-test analysis.

In general, the mean intake of energy (kcal), protein (gram) and fat (gram) were somewhat lower on gluten-free diet than in normal diet, whereas carbohydrate intake 
(gram) was slightly higher in gluten-free diet. However, the paired t-test results show that none of the differences in energy or macronutrient intakes were statistically significant.

\section{Discussion}

The present study shows that the composition of VOCs in exhaled breath changes as a result of a dietary intervention, a gluten-free diet. After multivariate analysis it appeared that a specific VOCs pattern containing 12 compounds can distinguish breath samples obtained after a 4-week gluten-free diet from samples obtained under normal dietary conditions. Some of these 12 compounds are chemically identified and although their physiological origin is still unclear it can be speculated that they are derived from common changes in metabolic processes. The change in the VOC profile of the subjects on a gluten-free diet with that of (the same) subjects on a normal diet has been found for all subjects. A striking finding is that this change is reversible - after finishing the gluten-free period the profile changes again to the profile as measured before the intervention, within the borders of normal intraindividual variation. Even though reversibility occurred in every case, the time for organism adjustment seems to be depended on yet unknown individual characteristics. The results suggest that some of the participants were less responsive to the gluten-free diet. Previous findings by Aziz et al., 2012 relate the heterogeneous response triggered by gluten to the different ways of handling gliadin intake by the immune system. In another study the heterogeneity of food intake response was explained as a consequence of an individual core metabolic phenotype [26]. Therefore, the heterogeneity of the gluten-free diet group might be related to diverse individual metabolic phenotypes and/ or diversity in managing gliadin intake by immune system.

The change in VOCs excretion in breath upon changing from a normal diet to a gluten-free diet and its reversibility might be explained in several ways. It is known from literature that the gut microbiota reversibly changes when a normal diet is temporarily replaced by a gluten-free diet. Fecal microbiota analysis of 10 healthy 
subjects, after following 4 weeks of gluten-free diet indicated that the number of 'healthy' gut bacteria decreased (Bifidobacterium, B. longum and Lactobacillus), while potentially unhealthy bacteria increased (E. coli and total Enterobacteriaceae) [16]. Drift of microbiota composition into less positive manner, might be explained by an extensive reduction in the intake of oligofructose and inulin during gluten-free diet. These fructans, provided in $81 \%$ through wheat and barley consumption, were reported to have prebiotic action and are known from promoting growth of favorable colonic bacteria [27-29]. In the research of Palma et al., gluten-free diet appeared to be associated with reduction of polysaccharide intake. Undigested carbohydrates are considered as a main source of energy for commensal microbiota, thus lowering the supply of these compounds might explain the drift of the gut microbial composition. However, though from the food questionnaires completed by the subjects in our study no decreased carbohydrate intake could be deduced, the composition of the total carbohydrate may be different.

A change in a host microbiota would be expected to be associated with a change of volatile metabolites produced by bacterial communities. When absorbed into the bloodstream they would reach host organs and become subject of further human metabolism and finally be excreted in urine or breath [30, 31]. From the compounds which we were able to identify, one might be related to a change in a composition of gut microbiota. 2-butanol is a product of one of the pathways of the lactic acid bacteria $(L A B)$ metabolism even though the main product of fermentation for all $L A B$ is lactic acid. From all kinds of bacterial groups, $L A B$ is thought to be the most associated with human lifestyle. Lactobacillus brevis has an ability to reduce 2,3butanediol to 2-butanol when accompanied by two proteins: diol dehydratase and alcohol dehydrogenase [32]. This strain occurs widely in nature, fermented food of plant and animal origin [33] but also in human intestinal flora [34].

Another explanation of the change in VOCs profile upon gluten-free diet may be found in the direct interaction of the gluten proteins with the gut wall. From celiac disease persons it is known that these proteins are involved in an inflammation reaction in the gut wall, leading to severe complaints. Although celiac disease has a 
prevalence of about $1 \%$ in the general population, it is not clear whether similar but less severe effects may occur in a larger part of the population. There is a raising prevalence of gluten-induced GI disorders in people with normal tTG, IgE levels, which therefore could not be classified as allergic or celiac disease. Yet, subjects have been affected by gluten containing food and showed health improvement after changing to a gluten-free diet [35]. This led to an increased interest in newly classified gluten enteropathy: non-celiac gluten sensitivity [36]. Exclusion of wheat protein complex from high-fat diet in mice showed protective effects against body weight, partially by provoking lipid mobilization and oxidation in adipose tissue, causing decreased accumulation of lipids. A gluten-free diet seemed to improve insulin sensitivity through suppression of the inflammatory profile. Animals fed a gluten-free diet showed lower macrophage infiltration and pro-inflammatory cytokine production, e.g., MCP-1/CCL-2, IL-6, TNF- $\alpha$ which supports the mechanism of gliadin-macrophages interactions [37, 38]. This finding is supported also by a research of Palma et al, where suppression of immune system by lowered production of pro-inflammatory cytokines: TNF and IFN-y was noticed in healthy subjects after following a gluten-free diet [16]. A light or latent present inflammation reaction might influence the VOCs composition and a change in a diet from normal to gluten-free would then be accompanied by a change in VOCs profile as well. So far, analysis of exhaled breath samples has proven its usefulness in recognizing conditions with elevated levels of oxidative stress and inflammatory processes. In this case a reduced inflammatory condition during a gluten-free diet should lead to a reduction of volatile products of inflammation in the form of lipid oxidation products. One of compounds we identified as being changed due to gluten-free diet - octane has been previously reported as a marker of oxidative stress and inflammation [39]. Our results showed that this alkane was significantly reduced after following a glutenfree diet. On the other hand, dodecanal, nonanoic acid and nonanal levels were increased on a gluten-free diet even though these are also potential markers of oxidative stress [40, 41]. Obviously more insight into the actual processes is necessary before reaching an end conclusion. We were not able to find a valid explanation for discriminant presence of two other identified compounds: 2-propyl1-pentanol and dihydro-4-methyl-2(3H)-furanone. Further research needs to be 
conducted to establish their biochemical origin and possible pathways of their involvement.

Performing breath analysis to study the influence of dietary interventions and the putative reversibility of their impacts could be used as a new potential tool in fields of nutrition and metabolism. 


\section{References:}

1. Nistal, E., et al., Differences of small intestinal bacteria populations in adults and children with/without celiac disease: effect of age, gluten diet, and disease. Inflammatory bowel diseases, 2012. 18(4): p. 649-56.

2. Boots, A.W., et al., The versatile use of exhaled volatile organic compounds in human health and disease. Journal of breath research, 2012. 6(2): p. 027108.

3. Kansal, A., Sources and reactivity of NMHCs and VOCs in the atmosphere: a review. Journal of hazardous materials, 2009. 166(1): p. 17-26.

4. Rouseff, R. and K. Cadwallader, Headspace techniques in foods, fragrances and flavors: an overview. Advances in experimental medicine and biology, 2001. 488: p. 1-8.

5. Wood, M., et al., Recent applications of liquid chromatography-mass spectrometry in forensic science. Journal of chromatography. A, 2006. 1130(1): p. 3-15.

6. Van Berkel, J.J., et al., A profile of volatile organic compounds in breath discriminates COPD patients from controls. Respiratory medicine, 2010. 104(4): p. 557-63.

7. Dallinga, J.W., et al., Volatile organic compounds in exhaled breath as a diagnostic tool for asthma in children. Clinical and experimental allergy : journal of the British Society for Allergy and Clinical Immunology, 2010. 40(1): p. 68-76.

8. Verdam, F.J., et al., Non-alcoholic steatohepatitis: A non-invasive diagnosis by analysis of exhaled breath. Journal of hepatology, 2012.

9. $\quad$ Altomare, D.F., et al., Exhaled volatile organic compounds identify patients with colorectal cancer. The British journal of surgery, 2013. 100(1): p. 144-50.

10. Garner, C.E., et al., Volatile organic compounds from feces and their potential for diagnosis of gastrointestinal disease. FASEB journal : official publication of the Federation of American Societies for Experimental Biology, 2007. 21(8): p. 1675-88.

11. Filipiak, W., et al., Dependence of exhaled breath composition on exogenous factors, smoking habits and exposure to air pollutants. Journal of breath research, 2012. 6(3): p. 036008.

12. Hornell, A., Effects of a gluten-free diet on gastrointestinal symptoms in celiac disease. The American journal of clinical nutrition, 2005. 81(6): p. 1452-3; author reply 1453.

13. Wen, S., et al., Structural genes of wheat and barley 5-methylcytosine DNA glycosylases and their potential applications for human health. Proceedings of the National Academy of Sciences of the United States of America, 2012. 109(50): p. 20543-8.

14. Hadjivassiliou, M., C.A. Williamson, and N. Woodroofe, The immunology of gluten sensitivity: beyond the gut. Trends in immunology, 2004. 25(11): p. 578-82.

15. Sollid, L.M., Coeliac disease: dissecting a complex inflammatory disorder. Nature reviews. Immunology, 2002. 2(9): p. 647-55.

16. De Palma, G., et al., Effects of a gluten-free diet on gut microbiota and immune function in healthy adult human subjects. The British journal of nutrition, 2009. 102(8): p. 1154-60.

17. Voedingscentrum, NEVO table 2006, S.N. Voedingsstoffenbestand, Editor. 2006.

18. Van Berkel, J.J., et al., Development of accurate classification method based on the analysis of volatile organic compounds from human exhaled air. Journal of chromatography. B, Analytical technologies in the biomedical and life sciences, 2008. 861(1): p. 101-7.

19. Walczak, B., Wavelets in Chemistry. 2000, Amsterdam: Elsevier Science.

20. Eilers, P.H.C. and B.D. Marx, Flexible Smoothing Using B-Splines and Penalized Likelihood. Statistical Science, 1996. 11: p. 89-121.

21. Nielsen, N.P.V., J.M. Carstensen, and J. Smedsgaard, Aligning of single and multiple wavelength chromatographic profiles for chemometric data analysis using correlation optimised warping. Journal of Chromatography A, 1998. 805(1-2): p. 17-35.

22. de Haan, J.R., et al., Interpretation of ANOVA models for microarray data using PCA. Bioinformatics, 2007. 23(2): p. 184-190.

23. Smolinska, A., et al., NMR and Pattern Recognition Can Distinguish Neuroinflammation and Peripheral Inflammation. Journal of proteome research, 2011. 10(10): p. 4428-4438.

24. Stanimirova, I., et al., Interpretation of analysis of variance models using principal component analysis to assess the effect of a maternal anticancer treatment on the mineralization of rat bones. Analytica chimica acta, 2011. 689(1): p. 1-7.

25. Geladi, P., et al., Principal Component Analysis of Multivariate Images. Chemometrics and Intelligent Laboratory Systems, 1989. 5(3): p. 209-220. 
26. Heinzmann, S.S., et al., Stability and robustness of human metabolic phenotypes in response to sequential food challenges. Journal of proteome research, 2012. 11(2): p. 643-55.

27. Jackson, F.W., Effects of a gluten-free diet on gut microbiota and immune function in healthy adult human subjects - comment by Jackson. The British journal of nutrition, 2010. 104(5): p. 773.

28. Gibson, G.R., Prebiotics as gut microflora management tools. Journal of clinical gastroenterology, 2008. 42 Suppl 2: p. S75-9.

29. van Loo, J., et al., On the presence of inulin and oligofructose as natural ingredients in the western diet. Critical reviews in food science and nutrition, 1995. 35(6): p. 525-52.

30. Hamer, H.M., et al., Functional analysis of colonic bacterial metabolism: relevant to health? American journal of physiology. Gastrointestinal and liver physiology, 2012. 302(1): p. G1-9.

31. Jacobs, D.M., et al., Non-digestible food ingredients, colonic microbiota and the impact on gut health and immunity: a role for metabolomics. Current drug metabolism, 2009. 10(1): p. 4154.

32. Lameiras, F., Butanol production in Lactic acid Bacteria, in Department of Chemical and Biological Engineering. 2012, Chalmers University of Technology Gothenburg, Sweden. p. 56.

33. Sanchez, I., L. Palop, and C. Ballesteros, Biochemical characterization of lactic acid bacteria isolated from spontaneous fermentation of 'Almagro' eggplants. International journal of food microbiology, 2000. 59(1-2): p. 9-17.

34. Gu, R.X., et al., Probiotic properties of lactic acid bacteria isolated from stool samples of longevous people in regions of Hotan, Xinjiang and Bama, Guangxi, China. Anaerobe, 2008. 14(6): p. 313-7.

35. Kaukinen, K., et al., Intolerance to cereals is not specific for coeliac disease. Scandinavian journal of gastroenterology, 2000. 35(9): p. 942-6.

36. Volta, U. and R. De Giorgio, New understanding of gluten sensitivity. Nature reviews. Gastroenterology \& hepatology, 2012. 9(5): p. 295-9.

37. Thomas, K.E., et al., Gliadin stimulation of murine macrophage inflammatory gene expression and intestinal permeability are MyD88-dependent: role of the innate immune response in Celiac disease. Journal of immunology, 2006. 176(4): p. 2512-21.

38. Soares, F.L., et al., Gluten-free diet reduces adiposity, inflammation and insulin resistance associated with the induction of PPAR-alpha and PPAR-gamma expression. The Journal of nutritional biochemistry, 2012.

39. Poli, D., et al., Exhaled volatile organic compounds in patients with non-small cell lung cancer: cross sectional and nested short-term follow-up study. Respiratory research, 2005. 6: p. 71.

40. Ashibe, B., et al., Dual subcellular localization in the endoplasmic reticulum and peroxisomes and a vital role in protecting against oxidative stress of fatty aldehyde dehydrogenase are achieved by alternative splicing. The Journal of biological chemistry, 2007. 282(28): p. 2076373.

41. Fuchs, P., et al., Breath gas aldehydes as biomarkers of lung cancer. International journal of cancer. Journal international du cancer, 2010. 126(11): p. 2663-70. 


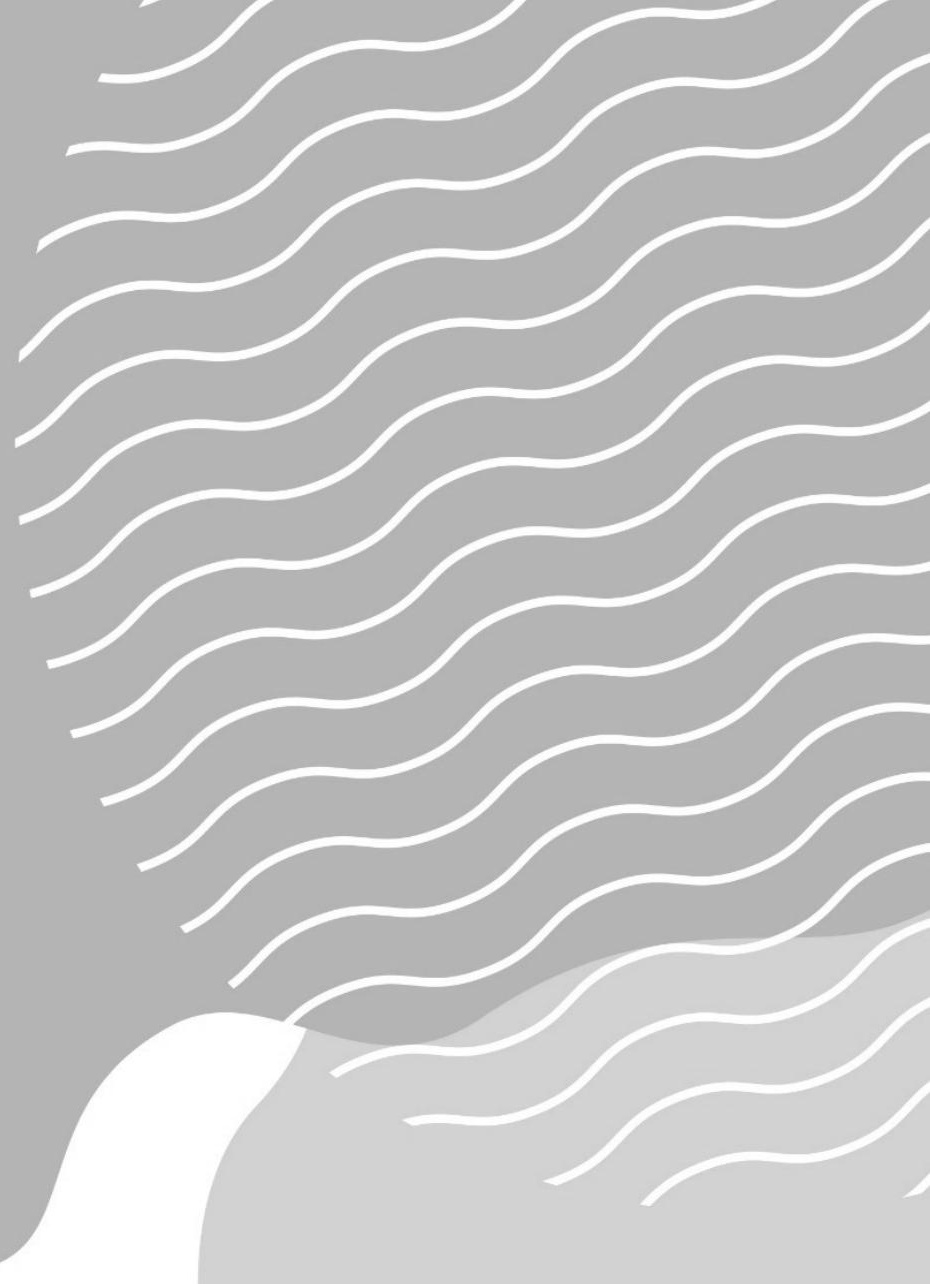

Chapter 4

Comparing patterns of volatile organic compounds exhaled in breath after consumption of two infant formulae with a different lipid structure: a randomized trial

A. Smolinska, A. Baranska, J. W. Dallinga, R.P. Mensink, S. Baumgartner, B.J.M. van de Heijning, F.J. van Schooten 


\section{Abstract}

Infant formulae have been used since decades as an alternative to or a complement to human milk. Human milk, the "gold standard" of infant nutrition, has been studied for its properties in order to create infant formulae that bring similar benefits to the infant. One of the characteristics of milk is the size of the lipid droplets which is known to affect the digestion, gastric emptying, and triglyceride metabolism. In the current study a concept infant milk formula with large, phospholipid coating of lipid droplets (mode diameter 3-5 $\mu \mathrm{m}$; NUTURIS, further described as "active"), was compared to a commercially available formula milk characterized by smaller lipid droplets, further described as "control" (both products derived from Nutricia). We investigated whether we could find an effect of lipid droplet size on volatile compounds in exhaled air upon ingestion of either product. For that purpose, exhaled breath was collected from a group of 29 healthy, non-smoking adult males before ingestion of a study product (baseline measurements, T0) and at the following time points after the test meal: 30, 60, 120, 180 and 240 min. Volatile organic compounds (VOCs) in breath were detected by gas chromatography-time-of-flight-mass spectrometry. Any differences in the time course of VOCs patterns upon intake of active and control products were investigated by regularized multivariate analysis of variance (rMANOVA).

The rMANOVA analysis revealed statistically significant differences in the exhaled breath composition 240 min after ingestion of the active formula compared to control product $(p$-value $<0.0001$ ) but did not show significant changes between active and control product at any earlier time points. A set of eight VOCs in exhaled breath had the highest contribution to the difference found at 240 minutes between the two formulas. A set of ten VOCs was different between baseline and the two formulae at T240 with $p$-value $<0.0001$. To our knowledge this is the first study that shows the ability of VOCs in exhaled breath to monitor metabolic effects after ingestion of infant formulae with different lipid structure. The statistically significant differences in compound abundance found between active and control formula milk may be related to: (i) specific differences in the digestion, (ii) absorption of lipids and proteins and (iii) assimilation of the products in the gut. 


\section{Introduction}

Human milk from a healthy mother is considered the most beneficial form of infant nutrition providing the best nutritional components, the most efficient delivery of nutrients and best transfer of immunological protection from a mother to her child. The aim of various producers of infant formula is to improve their current product concept by mimicking, as close as possible, native beneficial characteristics of human milk. The composition of human milk is very complex and dynamic and fluctuates over time. The content of human milk is associated with the maternal diet and phenotype [1,2]. Physiologically, fresh human milk contains fat globules that are on average about 2-10 times larger than those present in processed regular infant milk formulae. Moreover, the small fat globules present in infant formulae do not contain a phospholipid membrane that normally surrounds fat globules in human milk. In a study by Prentice et al.,[3], differences in dried blood spots lipid profiles were shown between human-fed versus formula-fed babies. Similarly, differences in Bifidobacteria count in fecal samples were found between formula-fed and human milk-fed infants [4]. Fat globule size influences the digestibility including gastric emptying and triglyceride metabolism [5,6]. Large fat globules are faster released from the stomach and thus result in earlier appearance and higher levels of triglycerides in blood [6]. In rats, this leads to a shift towards utilization of lipids in the metabolism of triglycerides for energy generation instead of incorporation and storage [6-8]. Small fat globules normally found in infant formulae remain in the stomach for a longer time and take more time to be absorbed in the gut. Therefore, they are less likely to be used for energy generation and might be used for fat storage to a large extent [7]. Early postnatal life is regarded as a critical period for the development of the metabolic system, which is programmed in early life in its functionality later in life. The relationship between infant feeding mode and long-term metabolic health could be related to the early-life growth trajectory. More specifically, rapid weight gain in early life has been related to higher risk of visceral adipose tissue in young children and adults $[9,10]$. A concept infant formula was developed with large, phospholipid coated lipid droplets (Nuturis) [11,12]. The beneficial effect of Nuturis infant formula was shown in a long-term experiment in mice [13]. Oosting 
and colleagues [13] showed that exposure to Nuturis early in life, helped mice to cope better with a moderate western style diet later in life, implicating a metabolic imprinting effect of Nuturis. The study demonstrated that the early exposure to the large, phospholipid coated lipid droplet concept diminished the accumulation of adipose tissue and improved the metabolic profile in mice during adolescence.

In the present study, the acute effect of Nuturis is examined in healthy male volunteers. The metabolic response upon ingestion of Nuturis (active) or standard infant milk formula (control) was tested and monitored in an exploratory, randomized, double-blind, controlled, crossover study up to $4 \mathrm{~h}$ post-ingestion as reported earlier by Baumgartner et al.,[14]. Post-meal samples of exhaled air (0-4 h) were collected and analyzed for volatile organic compounds (VOCs) as a read-out for metabolic responses. The analysis of the broad range of VOCs in exhaled breath gained popularity over the last years as a diagnostic and monitoring tool $[15,16]$. Various metabolic processes generate VOCs that are first released into the blood and then excreted via the lungs due to low solubility. In addition, the microbiome present in the gastrointestinal tract generates a number of volatile compounds during the metabolism of nutrients and xenobiotics [15]. Some of these compounds are excreted into faeces, while others enter the systemic circulation where they might be further modified by host metabolism and eventually excreted in breath. A single breath contains 300-500 VOCs occurring in nmol/l-pmol// concentrations and can be detected with several analytical techniques, including gas chromatography and mass spectrometry-based methods [17]. The number of VOCs in a series of breath samples may reach up to 3000-5000 due to inter-individual differences. Recent studies show that VOC profiles in exhaled air change due to, for instance, a glutenfree diet or the amount of fiber in the diet $[18,19]$. Therefore, we hypothesize that specific VOCs should be detected in exhaled breath after consuming two different formulas milk. In this study, we aim to explore the feasibility of exhaled air samples comparing the postprandial kinetics of two infant formulae, differing only in fat droplet size and phospholipid coating. 


\section{Materials and Methods}

\section{Study participants}

As reported previously [14] 29 healthy, non-smoking adult males with lactose and milk tolerance (age 18-25, BMI $20-25 \mathrm{~kg} / \mathrm{m}^{2}$ ) with no current and former known diseases and malfunctions (such as fat malabsorption, gastrointestinal malformation, hemophiliac, hepatitis B, HIV, high blood-pressure, hyperlipidemia, diabetes, flue, or severe diarrhea) and with no current use of any medications nor follow any specific diet participated in the study. The sample size of 29 was determined for a two-side effect $(p<0.05)$ of $20 \%$ and a discriminative power of $80 \%$.

All study participants gave written informed consent prior to inclusion. The study protocol was approved by the Maastricht University Medical Center+ (MUMC+) Ethics Committee (13-3-056.5/ab) and was in compliance with the revised Declaration of Helsinki (64th WMA General Assembly, Fortaleza, Brazil, 2013). The trial is registered at the Dutch Trial Registration, http://www.trialregister.nl (clinical trial number: NTR4463, registered on 10.03.2014) [14].

\section{Study design}

The study design was randomized, cross-over, and double-blind with allocation ratio 1:1, and performed in one center at Maastricht University, the Netherlands. The participants were assigned a random number at study entry after which the correspondingly numbered envelope was opened with the sequence of the study product the subject was receiving. The Excel was used for simple randomization of the study product for the participants. Note that the randomization was unknown to the investigator performing the study and to the site staff. The sequence was, however, known to the statistician who was responsible for making the randomization sequence, and the Supplies Manager who had to label the study products. The schematic diagram of the study is shown in Figure 1. The study consisted of two similar test days (day 1 at week 1 and day 1 at week 2). Each test started with a pre-test period (day -1 at week 1 and week 2) of one day preceding the actual study. At day -1 participants were not allowed to sport, drink alcohol, or 
eat high fat meals (i.e., $>10 \mathrm{~g}$ fat/meal). At the first day of the study (day 1 at week 1 ) fasting participants were asked to drink prepared shakes (4 times $100 \mathrm{~mL}$ within 1 min each and with 1 min interval). The subjects received Nuturis (called active) or control product in randomly assigned order. Both study products were infant milk formula (IMF) and contained an equal fat load of $37.5 \mathrm{~g}$ in $400 \mathrm{ml}$. The active product contained large phospholipid coating of lipid droplets [11,12], while the control product was commercially available IMF from Nutricia. The breath was collected before ingestion of a study product (baseline measurements, $\mathrm{T}=0$ ) and at the following time points after the test meal: 30, 60, 120, 180 and $240 \mathrm{~min}$. After the first day of the study a wash-out period of five days was considered sufficient to erase the effect of one test to another and to start the second analogous test day of the study.

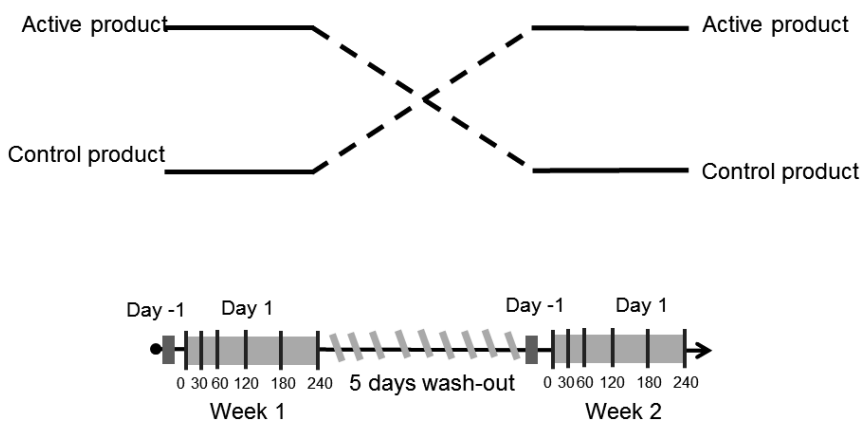

Figure 1. The schematic diagram of the study design.

\section{Exhaled air sampling and analysis}

At five time points after intake of the test meal (Figure 1), and one baseline time point before ingestion, exhaled air was collected from subjects [19]. Subjects exhaled breath into a $3 \mathrm{~L}$ Tedlar bag (Tedlar bag, SKC Ltd, Dorset, UK) whose content was 
thereupon transferred to a carbon-filled stainless steel sorption tubes (Markes International, Llantrisant Business Park, UK) where the breath compounds were trapped and stored until the actual analytical analysis (between 2-6 weeks at room temperature). The content of the sorption tubes was analytically analysed by Gas Chromatography coupled with time of flight-Mass Spectrometry (GC-tof-MS). The carbon tubes were placed inside the thermal desorption unit (Marks Unity desorption unit, Marks International Limited, Llantrisant, Wales, UK) and quickly heated to 270 ${ }^{\circ} \mathrm{C}$ in order to release all volatile molecules and to transport the released volatiles onto the GC. The volatile compounds trapped on the sorption tubes were released under a flow of Helium using automated thermal desorption. Next, the vaporous mixture was divided into two parts, $90 \%$ of the sample was recollected on an identical sample tube and $10 \%$ of the sample was transferred to an electrically cooled sorbent trap $\left(5^{\circ} \mathrm{C}\right.$ ) from which it was injected into a GC (column: RTX-5ms, $30 \mathrm{~m} \times 0.25 \mathrm{~mm}$ $5 \%$ diphenyl, 95\% dimethylsiloxane, film thickness $1 \mathrm{~mm}$, Thermo Electron Trace GC Ultra, Thermo Electron Corporation, Waltham, USA). The temperature of the GC was programmed as follows: first $40^{\circ} \mathrm{C}$ for 5 minutes then rose by $10^{\circ} \mathrm{C}$ every minute until $270^{\circ} \mathrm{C}$ was reached. This temperature was maintained for 5 minutes. Within the tof-MS part electron ionization at $70 \mathrm{eV}$ was applied with a $5 \mathrm{~Hz}$ scanning rate over a range of $\mathrm{m} / \mathrm{z} 35-350$. The tof-MS allows for compounds detection and identification based on their total mass spectrum. Tof-MS measures the time necessary to travel in a field free region from the ionization source and acceleration field to a detector plate. Because all ions have the same kinetic energy $\left(E=1 / 2 m v^{2}\right)$, ions with different mass-to-charge ratio ( $\mathrm{m} / \mathrm{z}$ values) are separated in the flight tube based on their velocity. Note, that due to practical limitations, i.e., absence of standard reference materials for a priori unknown $\mathrm{VOC}$, compounds are quantified using relative quantification, i.e., determining the response ratio between a VOC and other VOCs. The output of the GC-tof-MS consists of two dimensions: retention time and $\mathrm{m} / \mathrm{z}$ value and thus, for each measured compound in a breath sample, retention time and mass spectrum are available. These two parameters are also used in the chemical identifications of the VOCs that are selected after multivariate statistical analysis and are considered to contain valuable information [3]. Spectrum recognition using the National Institute of Standards and Technology library in combination with spectrum 
interpretation by an experienced Mass-Spectrometrist and identification based on retention times of compounds were used to obtain a putative chemical identification of the relevant VOCs.

\section{Data pre-processing and statistical analysis}

Data pre-processing of the raw data is implemented before the actual statistical analysis, as previously described [20]. Shortly, first the beginning and end of each chromatogram (retention time either $<1.3$ or $>23 \mathrm{~min}$ ) were removed because of noisy mass spectra at the beginning of the chromatograms and column bleeding at the end of each run. Then noise removal was performed via wavelets transformation and baseline correction by means of P-splines. Next step of data pre-processing is the creation of the peak list for each Total ion current (TIC) chromatogram allowing calculation of area under the peaks. Note that these areas are proportional to the relative amounts of measured compounds in exhaled air. Next, the area under the peaks for each TIC chromatograms were merged by combining the corresponding compounds based on retention time and similarities in mass spectra. To make the chromatograms comparable the final step of pre-processing involved PQN normalization [21].

The postprandial time course of VOCs in expired air after ingestion of the study products were compared and tested for differences. All statistical analyses were performed using Matlab R2014b.

Since each individual delivered 12 breath samples, intra-individual variation may obscure the information of interest. To diminish the influence of intra-individual variation centering per individual was performed. This allows converting all the relative compounds concentrations to fluctuations around zero for each individual instead of around the population mean. In other words, the biological mean for each individual is set to zero [22].

Before the actual statistical analysis, the presence of outliers is investigated by means of robust-Principal Component Analysis (r-PCA) [23]. The VOCs that are statistically different between active and control product over time and/or at a certain time point were found by means of regularized Multivariate Analysis of Variance (rMANOVA) for paired samples [24]. The rMANOVA technique is an extension of 
standard MANOVA applicable with poor ratio between number of samples $(n)$ and number of measured parameters ( $p)$, here volatiles in exhaled breath.

To visualize the differences, groupings, and trends between active and control nutrition product principal component analysis (PCA) was performed on the set of selected significant VOCs. PCA converts the multidimensional data space into a lowdimensional model plane using principal components (PCs). PCs are linear combinations of the originally measured compounds and can be summarized in score plots and loading plots. Score plot provides the summary and the relation of all measured samples in the space defined by PCs. In a loading plot the relation between measured compounds is displayed. The significance of the compounds was tested by means of the Wilcoxon Signed Rank Test [25] for paired samples, with Benjamini-Hochberg correction [26]. A significance level of p-value $<0.05$ was selected.

\section{Results}

\section{Data}

29 individuals delivered exhaled breath samples at five time points and at baseline leading to total number of 348 samples. The initial quality check of the data and $r$ PCA led to the removal of 35 samples. Therefore, from the total number of 348 samples, the statistical analysis was performed on 313 samples. In these 313 samples a total of 875 compounds was measured. Because the majority of individual VOCs was detected only in a limited number of samples the rule was applied that a compound was only kept for further analysis if it was present in at least $20 \%$ of the samples in one of the experimental groups or time point [19]. This led to a final data matrix containing 348 VOCs.

\section{Active vs. control products}

The rMANOVA models were performed on samples obtained at each corresponding time point for the two products (active and control). This analysis showed a significant difference between active and control product only at time point 240 (pvalue $<0.0001)$. None of the earlier time points revealed significant differences in 
VOCs profiles between the two formulae. In Figures 2A-B the averaged VOCs profiles for active and control products at baseline T0 and T240 are shown, respectively. As could be expected the VOCs profiles of both groups are similar (Figure 2A) at baseline, i.e., before ingestion of the formula. Four hours (240 minutes) after consuming the nutrition product the averaged VOCs profile revealed visible differences, as shown in Figure 2B. The largest differences can be seen in the chromatograms between RT of 16 and $19 \mathrm{~min}$.
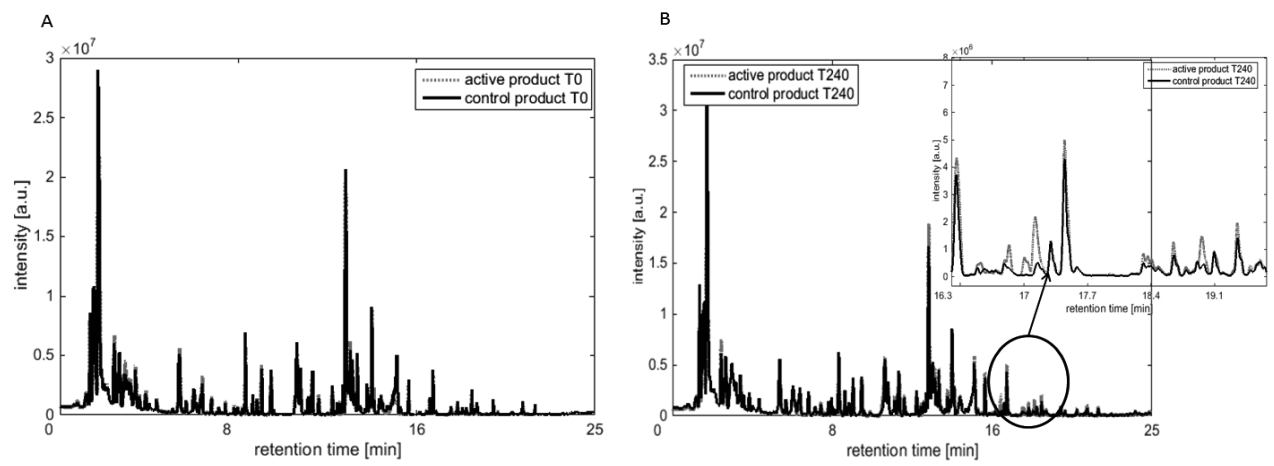

Figure 2. The averaged VOCs profiles for active and control products at: A) baseline TO, i.e., before consumption of the nutrition product; the VOCs profiles of active and control product are similar at the baseline. B) at T240, after consumption of the nutrition product; the VOCs profiles are different between the two nutritional products.

A set of eight VOCs was selected to be statistically significant ( $p$-value $<0.0001$ ) between active and control products by rMANOVA at time point 240. This set was re-analysed in a separate PCA analysis and the corresponding PCA score plot is shown in Figure 3. Each diamond and circle point corresponds to a breath sample of an individual consuming an active or control nutritional product. The PCA score plot shows differences between the two products along PC1 and PC2 explaining $48 \%$ of the total variance. Six samples from the control nutritional group seems to have a profile of compounds similar as those from the active group as they can be found in the cluster of samples of the active product group. The changes in the relative concentration of each of eight significant compounds is demonstrated in 
Table 1. Three compounds out of eight (2-methyl-propanoic acid, 2,4dimethylhexane and $\mathrm{C}_{5} \mathrm{H}_{7} \mathrm{COOH}$ ) point in the direction of active product, indicating higher concentrations of these breath compounds in the active group. The opposite is seen for the remaining compounds as shown in Table 1.

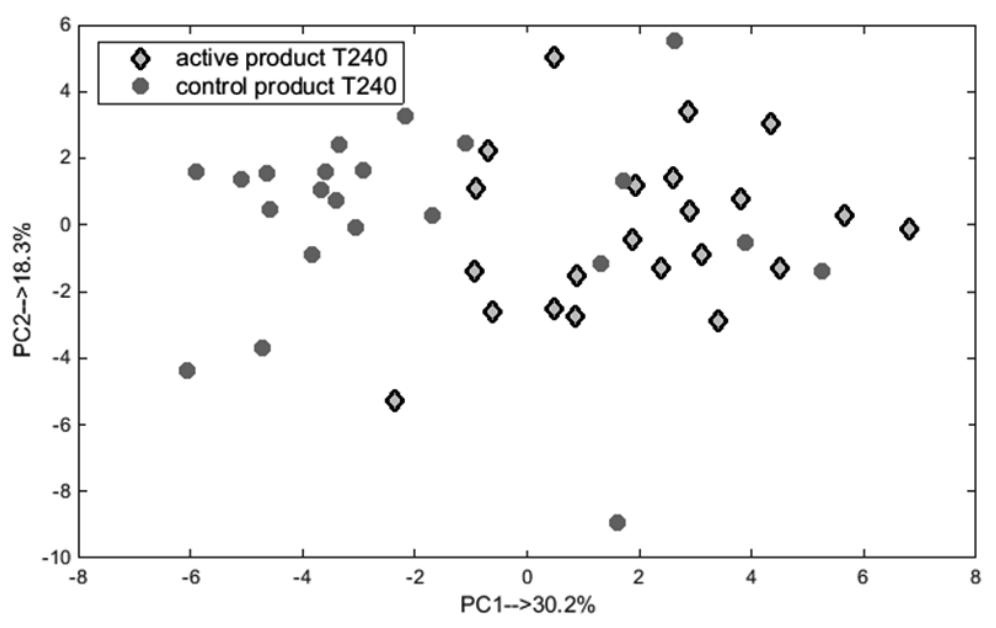

Figure 3. PCA analysis performed on 8 significant VOCs excreted in breath samples obtained at T240 of active and control products. The individuals are marked with respect to nutrition product (diamonds for active and circles for control products). The lines correspond to compounds used in the PCA analysis.

Table 1 shows the identified and unidentified VOCs and their relative concentrations change in breath samples obtained from individuals on active versus control product at T240. Up or down regulation of VOC concentrations is indicated as (+) or (-), respectively, with reference to control nutrition product at T240.

Though it is the combination of all 8 VOCs that yields high differences, we also analyzed the statistical importance of the 8 individual VOCs between both products using the Wilcoxon Signed Rank Test. Interestingly, all 8 compounds were statistically significant different, with a p-value $\leq 0.05$ (Table 1). 


\begin{tabular}{|c|c|c|c|}
\hline $\mathrm{Nr}$. & Putative identification & Relative change & p-value \\
\hline 1 & Isoprene & $(-)$ & 0,006 \\
\hline 2 & $\mathrm{C}_{8} \mathrm{H}_{18}$ branched $^{\#}$ & $(-)$ & 0,006 \\
\hline 3 & 2-methyl-propanoic acid & $(+)$ & 0,007 \\
\hline 4 & 2,4-dimethylhexane & $(+)$ & 0,006 \\
\hline 5 & 2- or 3-methylthiophene ${ }^{\#}$ & $(-)$ & 0,02 \\
\hline 6 & Decalactone & $(-)$ & 0,006 \\
\hline 7 & $\mathrm{C}_{5} \mathrm{H}_{7} \mathrm{COOH}^{\#}$ & $(+)$ & 0,007 \\
\hline 8 & 1,2,4-trimethylbenzene & $(-)$ & 0,02 \\
\hline
\end{tabular}

Table 1. List of 8 VOCs which changes in concentrations were significantly different between both products at T240. (-) indicates decrease in VOC concentration, while (+) indicates increase in active product compared to the control group. P-value indicates the statistical significance of the compounds. \# The exact isomer could not be determined.

The analysis by rMANOVA did not reveal significant differences between both products at earlier time points (i.e., T0, T30, T60, T120 and T180). The differences observed at T180 were on the border of being significant with a p-value of 0.054 . The PCA analysis performed on the set of 8 significant volatiles at T180 did not reveal clear clustering of the groups.

\section{TO vs. T240 for active and control product}

In a second approach, the rMANOVA analysis selected a set of 10 VOCs (p-value< 0.0001 ) that differ significantly between exhaled breath taken at baseline T0 and T240 (both nutrition products taken together). Two of these compounds were also part of the initial set of 8 VOCs that differentiated between active and control products at T240. Both sets of volatiles were combined, leading to a new set of 16 VOCs and PCA analysis of all T0 and T240 samples was performed on this combined set. The resulting PCA score plot is shown in Figure 4. The variance 
observed along PC1 (explaining 31\% of total variance) corresponds to differences in VOC profiles between T0 and T240 measurements (both products) while PC2, explaining $12 \%$ of variance represents the differences between active and control group at $\mathrm{T}=240$. For the comparison purpose two additional combinations of PCA score plots were performed, namely: (i) for samples obtained at T240 for active and control product using a set of 16 VOCs, and (ii) for samples obtained at T0 and T240 for active and control product using a set of eight VOCs. The corresponding PCS score plots can be found in the supplementary material (see Figure 1S-2S). In Figure $1 S$ and $2 S$, the active and control products create the distinct cluster of samples, indicating differences in volatile metabolites profile.

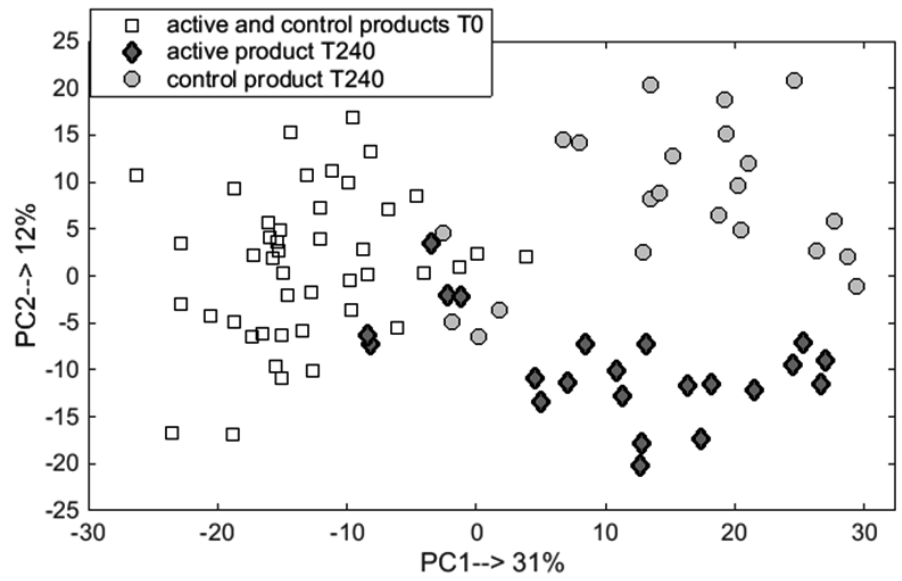

Figure 4. PCA scores plot for breath samples measured at baseline TO and T240 for active and control nutrition products based on 16 volatile compounds. The samples are coded with respect to time (square for T0) and nutrition product (diamonds for active and circles for control at T240).

As indicated before, a set of 10 VOCs was found to be statistically significant by rMANOVA and these VOCs contribute to the differentiation between baseline measurements and measurements at T240 (i.e., fasting versus postprandial) of both product groups. The relative importance and putative identification of each of these 10 volatile compounds is presented in Figure 5 . Four of these volatiles were more 
abundant in breath of individuals after consuming one of the products at T240 relative to the baseline measurements. The significance of the individual volatiles is shown in Figure 5. Nine out of 10 compounds were statistically significant with $p$ value $<0.0001$, while one compound had a $p$-value $<0.05$ (Figure 5).

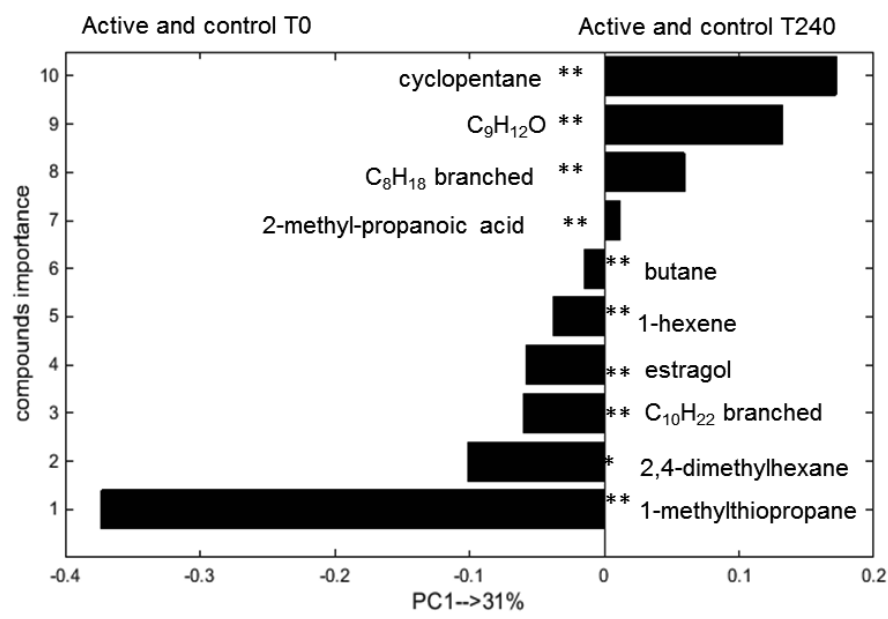

Figure 5. PCA loading bar plot depicting the relative importance of the 10 significant volatiles in the separation of all T0 and T240 breath samples for both products. Bar length is proportional to compound importance. Bars projecting to the left indicate greater abundance in breath samples taken at TO, while those projecting to the right indicate greater abundance in breath samples taken at T240. ${ }^{* *} p$ value $<0.0001$, ${ }^{*} p$-value $<0.05$. The exact isomers of $\mathrm{C}_{9} \mathrm{H}_{12} \mathrm{O}, \mathrm{C}_{10} \mathrm{H}_{22}$ and $\mathrm{C}_{8} \mathrm{H}_{18}$ could not be determined.

From the first set of 8 VOCs 7 were putatively chemically identified, while for the one remaining compound the exact isomer remained unknown. From the set of $10 \mathrm{VOCs}$, 8 were chemically identified, whereas for the remaining two the exact chemical formula remained unknown. Some compounds could not be chemically identified due to insufficient mass spectrum, overlap in the retention time or absence of mass spectrum in the library. 


\section{Discussion}

In this study we demonstrate changes of exhaled VOCs in breath after consumption of different infant formulae. This finding opens possibilities of alternative, noninvasive ways of investigating and monitoring postprandial effects of nutritional products, for instance, in infants and small children. We studied the effect of two infant milk formulae, active (Nuturis containing large phospholipid coating of lipid droplets) and control (commercially available IMF from Nutricia) product, up to 4 hours upon ingestion, in a group of healthy, non-smoking males on the postprandial profile of VOCs in exhaled air. We found that a limited number of eight VOCs to be statistically different between the active and control nutritional products at T240 only, not at earlier time points. In an adult person, the transit time for food to pass from oral intake and subsequent fecal excretion varies between 24-72 hours. In this course, the gastric emptying takes about 2-6 hours and food enters the small intestine where it takes about 3 hours to empty half of its content. Our results show that the largest difference in breath $\mathrm{VOC}$ composition between the two formulae was observed after 4 hours ( $p$-value $<0.0001$ ), i.e., the time that food enters the small intestine and nutrients might be absorbed there. Marginal significance was shown at earlier time point, i.e., T180 ( $p$-value=0.054). Although, the results showed statistical differences after 4 hours of consuming the test products, it is unknown till when the differences hold, since no further time points were sampled. Moreover, the differences shown here are demonstrated for healthy males and therefore it is not completely sure what the outcomes will be if infants are investigated.

The main study outcome reported by Baumgartner et al., [14] shows that consuming active product results in an earlier peak of plasma glucose, insulin levels and an earlier time to nadir in non-esterified fatty acids plasma concentrations. In addition, plasma triacylglycerol levels were slightly significantly elevated after 3 hours. The differences in these parameters and VOCs excretion after 3 to 4 hours might be explained by dissimilarities in absorption due to different fat globules and the phospholipid coating. Another finding of our study was that a set of ten VOCs in exhaled air was found to be statistically significant after 4 hours (T240) compared with before consumption. They might well reflect differences between the fasting 
stage and the satiation/digestion stage. In addition, we tested whether there are significantly different compounds between T0 and T240, separately, for active and control product (data not shown). In the set of most significant VOCs we found two compounds, 2-methyl-propanoic acid and $\mathrm{C}_{5} \mathrm{H}_{7} \mathrm{COOH}$, which were significantly different between active and control product at T240 as well contributing to the differentiation between T0 and T240 when active and control products were combined as one group. This indicates that those compounds might be specific to consuming of the active product.

We found the levels of cyclohexane and estragol to be significantly different between T0 and T240 measurements. Moreover, the levels of decalactone and 1,2,4-trimethylbenzene were significantly lower after consumption of active compared to the control product. The origin of these four compounds is not entirely certain but heterocyclic and aromatic compounds might be of dietary origin (mostly from plants) or air pollution [27]. The fat content of both active and control formulae consists of a blend of vegetable oils indicating that these heterocyclic and aromatic compounds are presumably originating from diet.

One of the selected compounds is 2-methylpropanoic acid (isobutyric acid), which was found to be statistically significantly higher in the active than in the control group at T240, and also significantly higher at T240 (combined groups) compared to T0 measurements. This compound is a structural isomer of butyric acid, which is a short chain fatty acid produced by colonic bacteria during fermentation processes of undigested carbohydrates [28,29]. 2-methylpropanoic acid belongs to the group of branched short chain fatty acids (BSCFAs), which might arise from dissimilation of amino acids [30]. Similarly, the levels of $\mathrm{C}_{5} \mathrm{H}_{7} \mathrm{COOH}$ (unsaturated fatty acid, for which the exact isomer could not be determined) was found to be significantly elevated in the active group compared to the control group. Although the formula milk is known to contain saturated and unsaturated fatty acids, the source of this compound in exhaled breath remains elusive.

The amount of 1-methylthiopropane was found to be lower after consumption of active or control product at T240 when compared to baseline measurements. 1- 
methylthio-propane passes into alveolar air from the blood, where it may originate from drug or diet [31]. However, in the current study the healthy volunteers did not take any drugs, and a plausible explanation of the sulfur compounds' source cannot be offered. Another sulfur-containing compound, 2(or 3)-methylthiophene, was found to be significantly lower in the active compared to the control group. Various sulfur-containing compounds were previously reported in breath, faeces, blood and urine [32-34] and their origins were linked to sulphate-reducing bacteria, Clostridia in the gut, vegetables, nut, bread and beer in the diet.

A large number of linear and branched hydrocarbons have been found in breath of healthy as well diseased individuals [16,33-35]. Unsaturated fatty acids consumed in our diet can be expected to create many hydrocarbons by chain cleavage and peroxidation [33]. Various hydrocarbons are stable end products of lipid peroxidation and have low solubility in blood, so within a few minutes upon their generation they will be excreted via breath. We found several branched and linear hydrocarbons to be different between T0 and T240 measurements as well as between the two study products. For instance, butane level was found to be reduced in active and control product at T240 in comparison to baseline measurements. Although, butane has been related to inflammatory processes occurring in the body [36], it is also known that it is produced by the gut microbiome [16].

Isoprene is one of the most abundant endogenous compounds found in exhaled air [37]. Isoprene is a by-product of cholesterol biosynthesis along the mevalonic acid pathway $[33,38]$. The level of isoprene in exhaled breath is proportional to the level of cholesterol in blood and therefore there has been a lot of investigation towards the use of breath isoprene analyses as a non-invasive diagnostic tool to measure blood cholesterol levels or cholesterol synthesis rate [39]. The link between isoprene and cholesterol metabolism is further sustained by the observation that there is an instantaneous decrease in isoprene excretion and sterol synthesis upon the administration of lovastatin, a lipid-lowering drug. Therefore, a diet which is rich in cholesterol is responsible for a reduction of isoprene in exhaled breath [40], hence monitoring the isoprene level in exhaled breath might be a monitoring marker of lipid and cholesterol status [16]. The level of isoprene was found to be significantly lower 
after the consumption of Nuturis (active product) in comparison to control product at T240. This result is particularly interesting since this would indicate that the sterol synthesis is lower in the active group in comparison to control. As the active meal contained $50 \%$ more cholesterol than the control meal, the endogenous cholesterol synthesis might be more inhibited, hence leading to lower isoprene co-production levels. Moreover, active product (Nuturis) contains larger sized fat droplets than control, causing its passage from the stomach to the duodenum to be faster, leading to an accelerated uptake of fat into the blood. This may suggest that after consuming the active product the plasma levels of lipid and cholesterol rise faster in comparison to the control product. This finding is in line with Baumgartner et al., [14] who reported faster increase in blood postprandial triacylglycerol after intake of active product in the same group of participants.

The use of GC-MS in the future implementation of the presented study in infants might be visible but selecting another approach might be more optimal. GC-MS methodology is an offline approach where content of exhaled breath is preconcentrated on sorption tube and later analytically measured [41]. An alternative technique, capable of detecting in sensitive and fast manner of various volatile metabolites in exhaled breath, is multi capillary column- ion mobility spectrometry. This methodology seems more applicable in infants since the content of breath can be measured directly and it takes about 5-10 minutes for complete analysis of exhaled air. This method has been successfully applied to various fields such as process control [42, 43], biological analysis [44, 45] and medical application [46, 47]. In the current study, the various time points were compared individually to each other. However, another approach could be applied, where a longitudinal aspect of the data is taken into account to look for the relevant compounds in exhaled breath that change over time. Recently, Hauschild et al., proposed a novel algorithm to first find the compounds that change over time and to define the slope of their change in exhaled breath [48].

In summary, we have shown the postprandial effect of two infant formulae (active and control) on the VOC composition of exhaled breath. Some of these VOCs may be indicative of specific differences in the digestion and absorption of the two studied 
products or the role of the gut microbiome in the assimilation of the products. Both should be subject of further investigations.

\section{Acknowledgment}

This research was financially supported by Nutricia Research, the Netherlands. The infant formulae investigated in this study were provided by Nutricia Research.

\section{Authors contribution}

Conceived and designed the experiments: AS, AB, JWD, BJMvdH, FJvS. Study volunteers' inclusion: RPM, SB. Data acquisition: AS and AB. Analysis and interpretation of data: $A S, A B, J W D$ and FJvS. Drafting of manuscript: $A S, A B$, JWD, BJMvdH, FJvS; Revision of the manuscript for important intellectual content: AS, AB, JWD, RPM, SB, BJMvdH, FJvS. Final approval of the version to be published: AS, AB, JWD, RPM, SB, BJMvdH, FJvS.

The authors declare no conflict of interest. 


\section{References}

1. Villasenor, A. et al. Breast milk metabolome characterization in a single-phase extraction, multiplatform analytical approach. Analytical chemistry 86, 8245-8252, doi:10.1021/ac501853d (2014).

2. Smilowitz, J. T. et al. The human milk metabolome reveals diverse oligosaccharide profiles. The Journal of nutrition 143, 1709-1718, doi:10.3945/jn.113.178772 (2013).

3. Prentice, P. et al. Lipidomic analyses, breast- and formula-feeding, and growth in infants. The Journal of pediatrics 166, 276-281 e276, doi:10.1016/j.jpeds.2014.10.021 (2015).

4. Klaassens, E. S. et al. Mixed-species genomic microarray analysis of fecal samples reveals differential transcriptional responses of bifidobacteria in breast- and formula-fed infants. Applied and environmental microbiology 75, 2668-2676, doi:10.1128/AEM.02492-08 (2009).

5. Armand, M. et al. Digestion and absorption of 2 fat emulsions with different droplet sizes in the human digestive tract. The American journal of clinical nutrition 70, 1096-1106 (1999).

6. Borel, P. et al. Digestion and absorption of tube-feeding emulsions with different droplet sizes and compositions in the rat. JPEN. Journal of parenteral and enteral nutrition 18, 534-543 (1994).

7. Michalski, M. C., Briard, V., Desage, M. \& Geloen, A. The dispersion state of milk fat influences triglyceride metabolism in the rat--a 13CO2 breath test study. European journal of nutrition 44, 436-444, doi:10.1007/s00394-005-0551-1 (2005).

8. Michalski, M. C., Briard, V., Michel, F., Tasson, F. \& Poulain, P. Size distribution of fat globules in human colostrum, breast milk, and infant formula. Journal of dairy science $\mathbf{8 8}$, 1927-1940, doi:10.3168/jds.S0022-0302(05)72868-X (2005).

9. Durmus, B. et al. Growth in foetal life and infancy is associated with abdominal adiposity at the age of 2 years: The Generation R Study (vol 72, pg 633, 2010). Clin Endocrinol 72, 856856 (2010).

10. Demerath, E. W. et al. Rapid Postnatal Weight Gain and Visceral Adiposity in Adulthood: The Fels Longitudinal Study. Obesity 17, 2060-2066, doi:10.1038/oby.2009.105 (2009).

11. Oosting, A. et al. A More Breast Milk-Like Infant Formula Reduces Excessive Body Fat Accumulation in Adult Mice. Pediatr Res 70, 837-837, doi:10.1038/pr.2011.1062 (2011).

12. Gallier, S. et al. A novel infant milk formula concept: Mimicking the human milk fat globule structure. Colloids Surf B Biointerfaces 136, 329-339, doi:10.1016/j.colsurfb.2015.09.024 (2015).

13. Oosting, A. et al. Size and phospholipid coating of lipid droplets in the diet of young mice modify body fat accumulation in adulthood. Pediatr Res 72, 362-369, doi:10.1038/pr.2012.101 (2012).

14. Baumgartner, S., van de Heijning, B. J. M., Acton, D. \& Mensink, R. P. Infant milk fat droplet size and coating affect postprandial responses in healthy adult men: a proof-of-concept study. Eur J Clin Nutr, doi:10.1038/ejcn.2017.50 (2017).

15. Buszewski, B., Kesy, M., Ligor, T. \& Amann, A. Human exhaled air analytics: Biomarkers of diseases. Biomed Chromatogr 21, 553-566, doi:10.1002/bmc.835 (2007).

16. Pereira, J. et al. Breath analysis as a potential and non-invasive frontier in disease diagnosis: an overview. Metabolites 5, 3-55, doi:10.3390/metabo5010003 (2015).

17. Boots, A. W. et al. The versatile use of exhaled volatile organic compounds in human health and disease. J Breath Res 6, 027108, doi:10.1088/1752-7155/6/2/027108 (2012).

18. Raninen, K. J. et al. Fiber content of diet affects exhaled breath volatiles in fasting and postprandial state in a pilot crossover study. Nutr Res 36, 612-619, doi:10.1016/j.nutres.2016.02.008 (2016).

19. Baranska, A. et al. Profile of volatile organic compounds in exhaled breath changes as a result of gluten-free diet. J Breath Res 7, doi:Doi 10.1088/1752-7155/7/3/037104 (2013).

20. Smolinska, A. et al. Current breathomics-a review on data pre-processing techniques and machine learning in metabolomics breath analysis. J Breath Res 8, doi:Doi 10.1088/17527155/8/2/027105 (2014).

21. Filzmoser, P. \& Walczak, B. What can go wrong at the data normalization step for identification of biomarkers? Journal of chromatography. A 1362, 194-205, doi:10.1016/j.chroma.2014.08.050 (2014).

22. Eriksson, L., Byrne, T., Johansson, E., Trygg, J. \& Vikströ, C. Multi- And Megavariate Data Analysis : Basic Principles And Applications (Umetrics AB, 2006). 
23. Stanimirova, I., Daszykowski, M. \& Walczak, B. Dealing with missing values and outliers in principal component analysis. Talanta 72, 172-178, doi:10.1016/j.talanta.2006.10.011 (2007).

24. Engel, J. et al. Regularized MANOVA (rMANOVA) in untargeted metabolomics. Anal Chim Acta 899, 1-12, doi:10.1016/j.aca.2015.06.042 (2015).

25. Wilcoxon, F. Individual comparisons of grouped data by ranking methods. Journal of economic entomology 39, 269 (1946).

26. Benjamini, Y. \& Hochberg, Y. Controlling the False Discovery Rate - a Practical and Powerful Approach to Multiple Testing. J Roy Stat Soc B Met 57, 289-300 (1995).

27. Alasalvar, C., Grigor, J. M., Zhang, D. L., Quantick, P. C. \& Shahidi, F. Comparison of volatiles, phenolics, sugars, antioxidant vitamins, and sensory quality of different colored carrot varieties. J Agr Food Chem 49, 1410-1416, doi:DOI 10.1021/jf000595h (2001).

28. Sagar, N. M., Cree, I. A., Covington, J. A. \& Arasaradnam, R. P. The interplay of the gut microbiome, bile acids, and volatile organic compounds. Gastroenterol Res Pract 2015, 398585, doi:10.1155/2015/398585 (2015).

29. Arora, T. \& Sharma, R. Fermentation potential of the gut microbiome: implications for energy homeostasis and weight management. Nutr Rev 69, 99-106, doi:10.1111/j.1753-

4887.2010.00365.x (2011).

30. De Preter, V., Van Staeyen, G., Esser, D., Rutgeerts, P. \& Verbeke, K. Development of a screening method to determine the pattern of fermentation metabolites in faecal samples using on-line purge-and-trap gas chromatographic-mass spectrometric analysis. Journal of chromatography. A 1216, 1476-1483, doi:10.1016/j.chroma.2008.12.095 (2009).

31. Berna, A. Z. et al. Analysis of Breath Specimens for Biomarkers of Plasmodium falciparum Infection. J Infect Dis 212, 1120-1128, doi:10.1093/infdis/jiv176 (2015).

32. Garner, C. E. et al. Volatile organic compounds from feces and their potential for diagnosis of gastrointestinal disease. Faseb J 21, 1675-1688, doi:10.1096/fj.06-6927com (2007).

33. Costello, B. D. et al. A review of the volatiles from the healthy human body. J Breath Res 8 , doi:10.1088/1752-7155/8/1/014001 (2014).

34. Mochalski, P. et al. Blood and breath levels of selected volatile organic compounds in healthy volunteers. Analyst 138, 2134-2145, doi:10.1039/c3an36756h (2013).

35. Turner, C. Techniques and issues in breath and clinical sample headspace analysis for disease diagnosis. Bioanalysis 8, 677-690, doi:10.4155/bio.16.22 (2016).

36. Miekisch, W., Schubert, J. K. \& Noeldge-Schomburg, G. F. Diagnostic potential of breath analysis--focus on volatile organic compounds. Clin Chim Acta 347, 25-39, doi:10.1016/j.cccn.2004.04.023 (2004).

37. de Lacy Costello, B. et al. A review of the volatiles from the healthy human body. J Breath Res 8, 014001, doi:10.1088/1752-7155/8/1/014001 (2014).

38. King, J. et al. Isoprene and acetone concentration profiles during exercise on an ergometer. $J$ Breath Res 3, 027006, doi:10.1088/1752-7155/3/2/027006 (2009).

39. Karl, T. et al. Human breath isoprene and its relation to blood cholesterol levels: new measurements and modeling. J Appl Physiol (1985) 91, 762-770 (2001).

40. Stone, B. G., Besse, T. J., Duane, W. C., Evans, C. D. \& DeMaster, E. G. Effect of regulating cholesterol biosynthesis on breath isoprene excretion in men. Lipids 28, 705-708 (1993).

41. Boots, A. W. et al. The versatile use of exhaled volatile organic compounds in human health and disease. J Breath Res 6, doi:10.1088/1752-7155/6/2/027108 (2012).

42. Vautz, W. \& Baumbach, J. I. Analysis of bio-processes using ion mobility spectrometry. Eng Life Sci 8, 19-25, doi:10.1002/elsc.200720221 (2008).

43. Baumbach, J. I. Process analysis using ion mobility spectrometry. Anal Bioanal Chem 384, 1059-1070, doi:10.1007/s00216-005-3397-8 (2006).

44. Prasad, S. et al. Analysis of bacterial strains with pyrolysis-gas chromatography/differential mobility spectrometry. Analyst 131, 1216-1225, doi:10.1039/b608127d (2006).

45. Junger, M. et al. Ion mobility spectrometry for microbial volatile organic compounds: a new identification tool for human pathogenic bacteria. Appl Microbiol Biot 93, 2603-2614, doi:10.1007/s00253-012-3924-4 (2012).

46. Baumbach, J. Medical applications of ion mobility spectrometry. International Journal for Ion Mobility Spectrometry 14, 137-137 (2011).

47. Westhoff, M. et al. Ion mobility spectrometry for the detection of volatile organic compounds in exhaled breath of patients with lung cancer: results of a pilot study. Thorax 64, 744-748, doi:10.1136/thx.2008.099465 (2009).

48. Hauschild, A. LOBRA - Modeling longitudinal Breath Data in R 2018). 


\section{Abstract}

Background: The diagnosis of Irritable Bowel Syndrome (IBS) is challenging because of its heterogeneity and multifactorial pathophysiology. No reliable biomarkers have been identified so far.

Aims: In this case-control study we investigated a novel application of breath analysis to distinguish IBS patients from healthy controls based on a set of volatile organic compounds (VOCs). Additionally, the VOC-biomarker set was correlated to self-reported gastrointestinal (GI) symptoms of subjects of the Maastricht IBS clinical cohort (MIBS) and of a general population cohort, LifeLines DEEP (LL DEEP).

Methods: Breath samples were collected from 170 IBS patients and 153 healthy controls in MIBS and from 1,307 participants in LL DEEP. Multivariate statistics were used to identify the most discriminatory set of VOCs in the MIBS cohort, and to find associations between VOCs and GI symptoms in both cohorts.

Results: A set of 16 VOCs correctly predicted $89.4 \%$ of the IBS patients and $73.3 \%$ of the healthy controls $(A \cup C=0.83)$. The VOC-biomarker set correlated moderately with a set of GI symptoms in the MIBS ( $r=0.55, p=0.0003)$ and LL DEEP cohorts $(r=0.54, p=0.0004)$. A Kruskal-Wallis test showed no influence from possible confounding factors in distinguishing IBS patients from healthy controls.

Conclusions: A set of breath-based biomarkers (16 VOCs) that distinguishes IBS patients from healthy controls was identified. The VOC-biomarker set correlated significantly with GI symptoms in two independent cohorts. We demonstrate the potential use of breath analysis in the diagnosis and monitoring of IBS, and a possible application of VOC-analyses in a general population cohort. 


\section{Introduction}

Irritable Bowel Syndrome (IBS) is a functional gastrointestinal (GI) disorder characterized by abdominal pain and altered bowel habits. IBS has a high prevalence of up to $15 \%$ among the general population in Western countries [1]. It is a heterogeneous disorder with a multifactorial pathophysiology including alterations in e.g., intestinal motility, visceral perception, intestinal microbiota, barrier function, low-grade immune activation, and psychological factors [2]. The diagnosis of IBS is symptom-based, using the Rome III criteria [3] and objective and validated biomarkers are lacking [4]. When available, the ideal biological marker should differentiate IBS patients from healthy individuals and from patients with organic GI diseases, such as inflammatory bowel diseases (IBD). Biomarkers for IBS should not only provide insight into the pathophysiological mechanisms that generate symptoms but should also correlate with symptom severity and thereby may be helpful in quantifying efficacy of therapeutic interventions in IBS [5].

Searches for individual biomarkers in IBS have failed so far, mainly because of the inability to capture the complexity and heterogeneity of IBS and the variation in symptoms [5, 6]. Therefore, we propose a more holistic approach. Using multiple characteristics and factors simultaneously, we intend to cover the full heterogenic spectrum of IBS. Analysis of exhaled breath is a promising and non-invasive tool in disease diagnosis and monitoring [7-12]. Hundreds of volatile organic compounds (VOCs) are present in human exhaled breath, of which the majority is comparable between individuals. Some compounds, however, differ significantly according to pathophysiological processes and metabolic differences and may indicate certain states of health or disease. Volatiles originate from several sources including inflamed tissue, normal and altered metabolic pathways of both the host and its microbiome. Upon formation, VOCs reach the blood-lung barrier through the blood stream and, due to their low solubility, are easily and rapidly excreted via the airways. A recent pilot study successfully tested VOCs biomarkers in paediatric inflammatoiry bowel disease [12]. Furthermore, we have recently demonstrated that a set of VOCs can separate healthy controls and patients with active Crohn's disease and Crohn's 
disease in remission in a real-life cohort [9]. Our findings are indicative for the potential of VOCs analyses in monitoring disease activity in this type of inflammatory bowel disease. However, wheather VOCs analyses have additional value in the diagnostic process and follow-up of IBS remains unlclear.

The aim of the present study was to use VOC analysis of exhaled breath to identify the presence of IBS and to search for correlations between VOCs and GI symptoms in both IBS patients and individuals from a general population cohort. More specifically, our research aims were:

1. To determine and validate a specific set of VOCs that distinguishes IBS patients from control subjects without GI complaints, using the clinical Maastricht IBS cohort (MIBS).

2. To test whether our set of VOCs correlates with GI symptoms in the MIBS cohort.

3. To test a possible application of the VOC biomarker set in a general population cohort, by testing whether our set of VOCs relates to more pronounced GI symptoms reported by subjects in a general population cohort (LL DEEP cohort).

\section{Materials and methods}

\section{Study participants}

Two cohorts were included into this study. The Maastricht IBS (MIBS) cohort was used for the aim 1 and 2, as it represents a well-defined group of IBS subjects and also age and gender matched controls, free of GI complaints. Aim 3 was accomplished by inclusion of a general population cohort, the LL DEEP cohort. All study participants gave written informed consent prior to inclusion. Study protocols were approved by the Maastricht University Medical Center+ (MUMC+) and University Medical Centre Groningen (UMCG) Ethics Committees, respectively, and were in compliance with the revised Declaration of Helsinki (64th WMA General Assembly, Fortaleza, Brazil, 2013). The MIBS cohort was registered in the US National Library of Medicine (http://www.clinicaltrials.gov, NCT00775060). 


\section{MIBS cohort}

Breath samples were collected from 170 well-phenotyped IBS patients and 153 age and gender matched healthy volunteers without GI complaints, who participated in a large clinical cohort study (MIBS) on the phenotypic and genotypic characterization of patients with IBS [13].

IBS patients, with the age ranging from 18 to 75 years, had been included in the cohort between September 2009 and January 2014 via the GastroenterologyHepatology outpatient clinic of Maastricht University Medical Centre+ (MUMC+), a secondary and tertiary referral centre, and via general practitioners (GPs). All patients were diagnosed according to the ROME III criteria and were examined by a gastroenterologist or GP. GI endoscopy, abdominal imaging and/or blood and faecal analyses were performed to exclude organic disease when indicated. Patients with a history of abdominal surgery, apart from appendectomy, laparoscopic cholecystectomy, or hysterectomy, were excluded. Age and gender matched healthy controls were enrolled via public advertisements and were subjected to a detailed medical interview to exclude the presence of $\mathrm{Gl}$ disorders and current or previous $\mathrm{GI}$ complaints or symptoms.

All participants filled in questionnaires on demographics and lifestyle. Their GI symptoms were assessed by an end-of-day GI symptom diary recorded for two weeks [13]. Mean 14-day scores per symptom were used for the analyses. Data on medication use and medical history were self-reported and cross-checked with hospital medical records.

\section{LL DEEP cohort}

Adult participants were enrolled from LifeLines, a large general population cohort in the Netherlands [14]. In a subgroup of 1307 non-selected participants of this cohort, exhaled breath samples were collected, along with a 7-day GI symptom diary, identical to the 2-week GI symptom diary used in the MIBS cohort. Information on clinical diagnoses of IBS was not available for these subjects. This sub-cohort of Lifelines (i.e., LL DEEP: LifeLines Detailed Extensive Examination of Participants) 
has been used for the current study and the study design of LL DEEP has been described in detail elsewhere [15].

\section{Sample collection and analysis}

Each participant inflated a 3 L Tedlar Bag (SKC Ltd, Dorset, UK) with no specific instructions on the method of exhaling. For each cohort, all samples were collected in the same room. To avoid content alterations, the VOC contents of each bag was captured within an hour in stainless-steel two-bed sorption tubes, filled with carbograph 1TD/Carbo-pack X (Markes International, Llantrisant, UK) and stored at room temperature until analysis [16]. Breath samples were analysed by thermal desorption-gas chromatography combined with time-of-flight mass spectrometry (TD-GC-tof-MS), as previously described [16].

\section{Statistical analysis}

Raw GC-tof-MS data were pre-processed to diminish the effects of noise, column bleeding, and chromatographic drift, as described previously [17].

The differences in the baseline characteristics were tested using independent samples T-test (for continuous normally distributed data) and Pearson Chi-squared test (for dichotomous data).

Aim 1: Search for VOCs biomarker panel

Random Forest (RF) was used to determine a set of volatile organic compounds that best separated IBS patients from controls in the MIBS cohort [17]. Therefore, chromatograms of IBS patients and the healthy control group were divided into a training ( $\pm 80 \%$ of the total population) and an independent validation set $( \pm 20 \%$ of the population) [18] using the Duplex algorithm [19]. We included 123 subjects of each group in a training set to find discriminatory VOCs and build a RF model. The remaining 47 IBS patients and 30 controls were used as an internal validation set to test the predictive accuracy of the selected VOCs. The receiver operating 
characteristic $(\mathrm{ROC})$ curve was used to demonstrate the performance of the RF classification model for the validation set. For each sample, RF delivered a probability of it belonging to the IBS or control group. These probabilities were used as a range of cut-off values to calculate the sensitivity and specificity presented in a ROC curve. Within the training set we applied the bootstrap aggregating technique which is a sampling approach based on random selection of samples with replacement. The main advantage of bootstrap aggregating is the improvement of stability and accuracy of the classification model [20]. This was used for model optimization and the most discriminatory VOCs selection. Note that RF model was validated twice. The first validation is a standard part of RF classification model using bootstrap aggregating technique. The second validation was incorporated via an independent validation set. We did not apply commonly used cross-validation for estimating the power of the classification model because it leads to optimistic bias (i.e., too low error estimate) [21].

For visualization, we performed Principal Component Analysis (PCA) on the proximity matrix obtained from the RF model built on the set of discriminatory VOCs [17].

\section{Influence of subjects' characteristics and importance of individual VOCs}

Potential contribution of the external factors defined by subjects' characteristics, to the separation by the set of 16 VOCs, was first checked by PCA score plots. To test whether any of the individual significant VOCs and the discriminatory profiles was statistically influenced by external factors (i.e., diet, smoking habit, alcohol consumption, symptoms of anxiety or depression, use of dietary supplements and use of medications indicated, all presented in Table 1), we then combined a KruskalWallis test with a Benjamini-Hochberg post-hoc correction for multiple testing with regularized multivariate analysis of variance $[22,23]$. 
Correlation between the VOC set and symptoms in MIBS (Aim 2) and LL DEEP population (Aim 3)

Linear correlations between the most discriminatory set of VOCs and a set of symptoms including all items of the GI symptom diary were tested in the MIBS and in the general population LL-DEEP cohort, using canonical correlation analysis (CCA) [24] which is an extension of bi-variate correlation. CCA calculates a linear correlation that best explains the variability both within and between the biomarker panel and the various $\mathrm{Gl}$ symptoms.

\section{VOCs identification}

The set of VOCs was tentatively identified by means of spectrum recognition, using the NIST (National Institute of Standards and Technology) library in combination with expert interpretation.

\section{Results}

\section{Baseline characteristics}

Baseline characteristics of the MIBS cohort are given in Tables 1 and 2. Although, IBS patients and healthy controls exhibit several differences in lifestyle, intake of supplements and drugs intake, no differences were observed in demographic characteristic and use of specific diets (Table 1). As expected, IBS patients did reveal higher GI symptom scores in comparison to healthy controls (Table 2). In table 3, the characteristics of LL DEEP cohort have been shown. The demographic features of LL DEEP cohort resemble both groups of MIBS. The mean GI symptom scores are more comparable to healthy controls then IBS patients of the MIBS cohort, which is in line with the fact that LL DEEP is a general population cohort. 


$\begin{array}{cc}\text { Healthy controls } & \text { IBS patients } \\ (n=153) & (n=170)\end{array}$

Demographic characteristics

\begin{tabular}{l|c|c}
\hline Age (mean years \pm SD) & $43.7 \pm 19.1$ & $42.9 \pm 16.7$ \\
\hline Female sex $(\%)$ & 63.1 & 72.4 \\
\hline BMl (mean kg/m² $\mathrm{SD})$ & $24.0 \pm 4.0$ & $24.9 \pm 4.4$ \\
\hline
\end{tabular}

Lifestyle parameters

\begin{tabular}{|c|c|c|}
\hline Current smoker (\%) & 7.6 & $25.3^{\star * *}$ \\
\hline Current or previous smoker (\%) & 38.6 & $51.3^{\star \star *}$ \\
\hline Alcohol intake: >0 units/week (\%) & 81.1 & $63.0^{* * *}$ \\
\hline
\end{tabular}

Use of medication, 2 weeks prior to participation in study

\begin{tabular}{|c|c|c|}
\hline Use of any medication (\%) & 41.6 & $78.7^{\star \star *}$ \\
\hline $\mathrm{PPI}(\%)$ & 2.0 & $20.2^{\star * *}$ \\
\hline SSRI (\%) & 2.7 & $9.2^{*}$ \\
\hline Prokinetic drugs (\%) & 0.0 & $3.7^{*}$ \\
\hline Laxatives (\%) & 0.6 & $17.8^{* * *}$ \\
\hline Antidiarrheal drugs (\%) & 0.0 & $4.3^{*}$ \\
\hline NSAID (\%) & 10.1 & 9.8 \\
\hline Antihypertensive drugs (\%) & 12.8 & 16.6 \\
\hline Statins (\%) & 5.4 & 9.8 \\
\hline \multicolumn{3}{|c|}{ Dietary habits } \\
\hline Current use of any diet (\%) & 9.4 & 15.4 \\
\hline Use of dietary supplements (\%) & 25.3 & $44.6^{\star *}$ \\
\hline
\end{tabular}

Table 1. Demographics and lifestyle of the MIBS cohort. 


\begin{tabular}{l|c|c}
\hline \multicolumn{2}{c}{ Gl symptom scores, 14-day mean \pm SD, 1-to-5 point scale } \\
\hline Abdominal pain & $1.1 \pm 0.2$ & $2.2 \pm 0.8^{* * *}$ \\
\hline Abdominal discomfort & $1.1 \pm 0.2$ & $2.4 \pm 0.8^{* * *}$ \\
\hline Bloating & $1.1 \pm 0.2$ & $2.2 \pm 0.9^{* * *}$ \\
\hline Belching & $1.1 \pm 0.3$ & $1.6 \pm 0.7^{* * *}$ \\
\hline Nausea & $1.0 \pm 0.1$ & $1.6 \pm 0.8^{* * *}$ \\
\hline Flatulence & $1.3 \pm 0.5$ & $2.2 \pm 0.8^{* * *}$ \\
\hline Constipation & $1.1 \pm 0.2$ & $1.4 \pm 0.6^{* * *}$ \\
\hline Diarrhoea & $1.0 \pm 0.1$ & $1.5 \pm 0.7^{* * *}$ \\
\hline
\end{tabular}

Table 2. GI symptom scores of the MIBS cohort. The 14-days end-of-day diary scores; 14-day means are presented. Differences between the two groups tested with the independent samples T-test. * $p<$ $0.05 ;{ }^{* *} p<0.01 ;{ }^{* \star *} p<0.001$.

\section{Demographical \& lifestyle characteristics $(n=1307)$}

\begin{tabular}{l|c}
\hline Age (mean years $\pm \mathrm{SD})$ & $45.4 \pm 13.5$ \\
\hline Female sex $(\%)$ & 58.2 \\
\hline BMI (mean kg/m² SD) & $25.5 \pm 4.4$ \\
\hline Current smoker $(\%)$ & 19.6 \\
\hline
\end{tabular}

GI symptom scores, 7-day mean \pm SD, 1-to-5 point scale

\begin{tabular}{l|c}
\hline Abdominal pain & $1.2 \pm 0.4$ \\
\hline Abdominal discomfort & $1.3 \pm 0.5$ \\
\hline Bloating & $1.5 \pm 0.6$ \\
\hline Belching & $1.1 \pm 0.4$ \\
\hline Nausea & $1.1 \pm 0.3$ \\
\hline Flatulence & $1.8 \pm 0.7$ \\
\hline Constipation & $1.2 \pm 0.4$ \\
\hline Diarrhoea & $1.1 \pm 0.3$ \\
\hline
\end{tabular}

Table 3. Baseline characteristics of the LL DEEP cohort. The 7-days end-of-day diary scores; 7-day means are presented. 


\section{Preparation of MIBS and LL DEEP data}

In the MIBS cohort (170 IBS patients and 153 healthy controls), we obtained 323 chromatograms by GC-tof-MS with a total of 587 different VOCs, each compound present in at least $20 \%$ of either IBS or healthy controls.

For LL DEEP, a total of 1307 chromatograms were measured, showing 3283 VOCs in total. No reduction in VOCs was applied in this group, as we evaluated the application of the identified set of VOCs in this LL DEEP cohort.

\section{Data analysis}

Search for VOC biomarker panel in the MIBS cohort (aim 1)

RF identified a group of 16 VOCs with best discriminatory power between IBS patients and healthy controls in the training set of the MIBS cohort. They were then tested on the independent validation set of the remaining 47 breath-o-grams of IBS patients and 30 of healthy controls, resulting in a sensitivity of $89.4 \%$ and a specificity of $73.3 \%$, with a positive predictive value of $84 \%$ and a negative predictive value of $81.5 \%$. The corresponding ROC curve, with the area under the curve (AUC) of 0.83 , is shown in Figure 1. PCA was then performed on the proximity matrix obtained from the RF model built on the 16 discriminatory VOCs. The corresponding score plot (Figure 2) shows the distributions of the breath-o-grams in the training set (red squares for IBS and blue circles for healthy controls) and in the validation set (black stars for IBS and black circles for healthy controls). There is a visible separation between the IBS patient and healthy control breath-o-grams in training set, but more importantly also in validation set. The partial overlap between the groups indicates that some IBS patients have VOC profiles more similar to healthy controls and vice versa, but these could not be related to either medical history or baseline clinical characteristics of these subjects. Furthermore, the overall variation was less in the healthy controls than in the IBS group. 


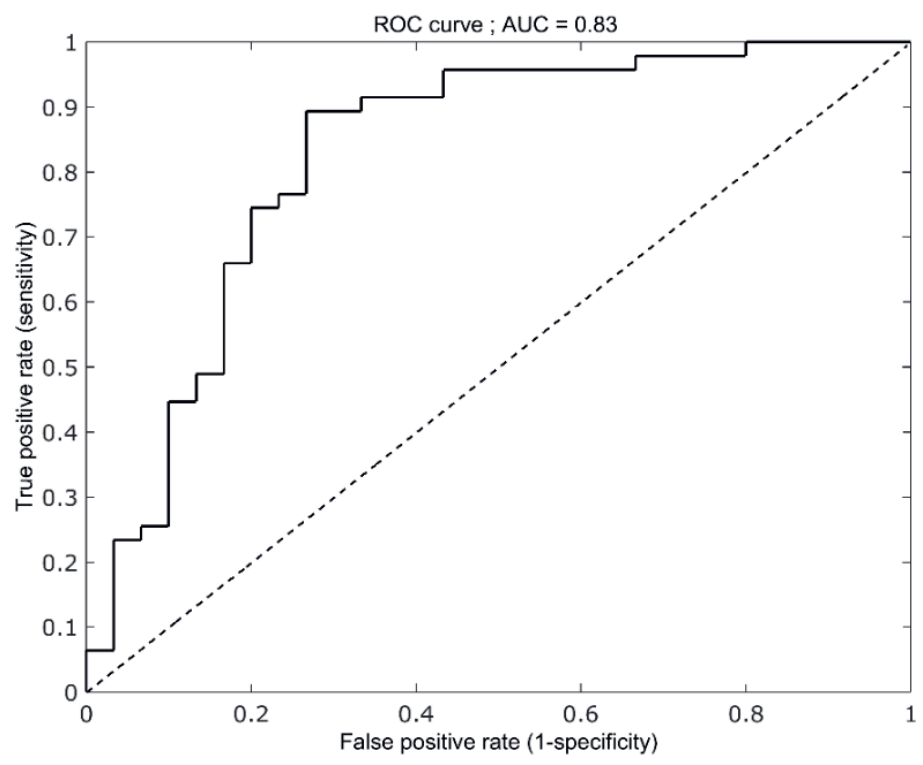

Figure 1. ROC curve performed on the validation set, with area under the curve $(A \cup C)=0.83$.

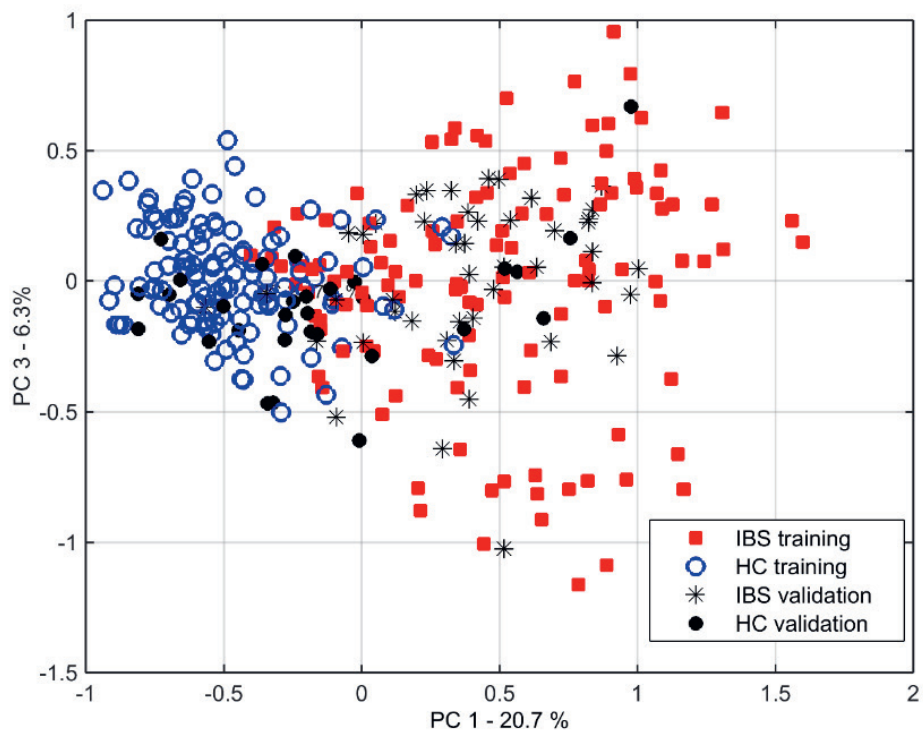

Figure 2. PCA score plot, on a proximity matrix obtained from the Random Forest model built on 16 selected VOCs. Each point is a single chromatogram. 
The importance of the selected set of VOCs in discriminating IBS patients from healthy controls and their relative abundance trend in IBS patients are shown in Figure 4 . The length of the bar in the Figure 4 indicates the importance of the compound in discriminating IBS from healthy controls. The relative concentrations of VOCs with a positive value are increased in the IBS group, and vice versa.

\section{Influence of subjects' characteristics}

To ensure that the separation between the healthy controls and IBS patients (see Figure 2) was not related to other factors, we displayed all available information on the same score plot. No associations or trends were observed in relation to age, gender, smoking habits, use of dietary supplements, alcohol, a specific diet, and medication, or the presence of depressive and/or anxiety symptoms. PCA score plots indicating the relation between VOCs and eight external factors (diet, smoking, age, gender, increased symptoms of anxiety or depression, intake of supplements and use of medication), together with the reference plots indicating the distribution of IBS patients and healthy controls, are shown in Figure 3. Then all abovementioned factors were analysed using the Kruskal-Wallis test combined with the BenjaminiHochberg for each selected VOC. The set of discriminatory VOCs was tested with rMANOVA. No significant differences were observed between the levels of individual VOCs as well as for the discriminatory set and the studied factors.

\section{Importance of individual VOCs in the discriminative set}

Even though it is the combination of all 16 VOCs that yields that high discriminative power, we also analysed the relative importance of the 16 individual discriminatory VOCs between the IBS and control groups using the Kruskal-Wallis test. Twelve out of 16 compounds were statistically significant, with a $p$-value $\leq 0.05$ (Figure 4 ). 

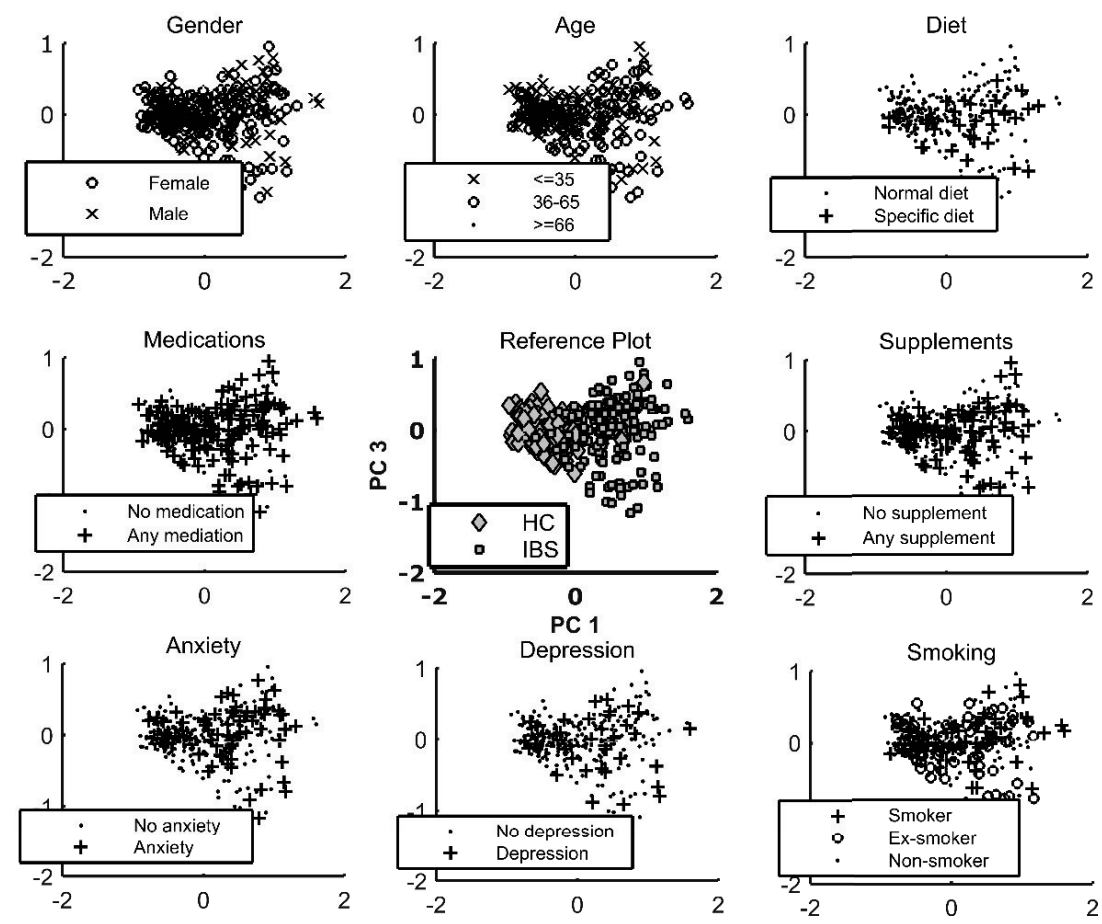

Figure 3. $P C A$ score plots, defined by $P C 1$ and $P C 3$, on a proximity matrix obtained from the Random Forest model built on 16 selected VOCs, with the reference plot in the middle. Each point is a single chromatogram, with points shaped by external factors. For some factors (medication, supplement use and diet), the chromatograms were shape-coded if the subject was on any medication vs. no medication, on any supplement vs. no supplements, and when following a specific diet (vegetarian, gluten-free, etc.) vs. a normal diet. 


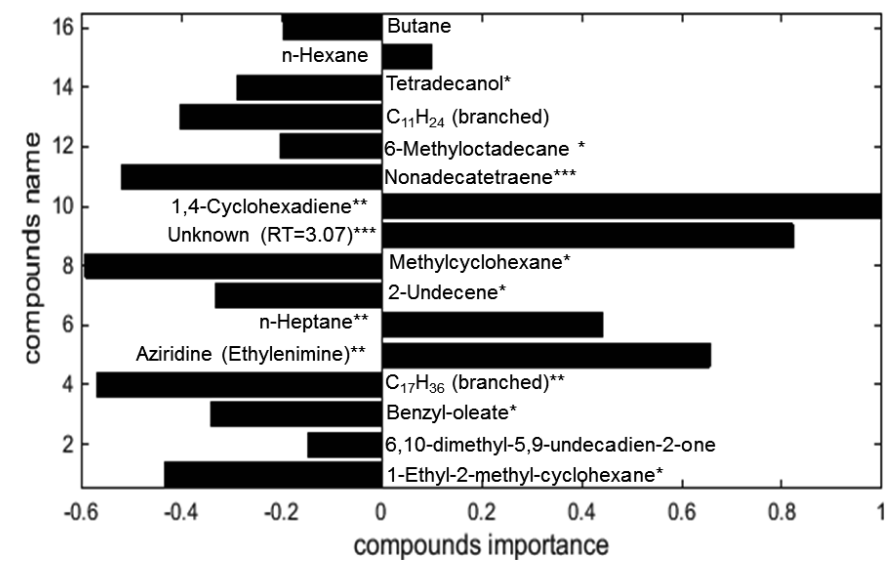

Figure 4. The importance of the selected VOCs in discriminating IBS and healthy controls. The length of the bar, i.e., positive, or negative values, indicates the relative importance of the specific VOC within the set. The relative concentrations of VOCs with a positive value are elevated in the IBS group, and vice versa for the negative VOCs.

Correlation between VOC set and symptoms in MIBS cohort (Aim 2)

We found a significant correlation of $r=0.55(p=0.0003)$ between the set of 16 VOCs and the set of following symptom scores: abdominal pain, discomfort, flatulence, and belching, which were assessed by the 14-day end-of-day GI symptom diary; for all symptoms 14-day mean scores were used (see also Table 2). No correlations were found between the VOCs and the other GI symptom scores measured.

Application of the biomarker panel in the general population LL DEEP cohort (Aim 3)

Similarly, we investigated the correlation between the discriminatory set of VOCs and the set of eight available symptoms from the 7-day end-of-day GI symptom diary available for the LL DEEP cohort (Table 3). Mean symptom scores were very low as many general population subjects do not report any symptom. Therefore, only subjects with a 7-day mean scores for abdominal pain or discomfort above 1.5 (on a 
1-to-5 point scale), were used in the correlation analysis. A mean score of 1.5 or higher indicates the subject had at least 2 days moderately severe or 4 days slightly severe GI symptoms during those 7 days. This cut-of value of 1.5 was further supported by data of the MIBS cohort in which $95 \%$ of healthy controls had end-ofday diary mean-scores below 1.5 for these Gl symptoms and was found in 239 subjects of the LL DEEP cohort. In this subgroup, a moderate and significant correlation was found between our set of VOCs and the following set of symptoms: abdominal pain, flatulence, belching, bloating, diarrhoea, and nausea $(r=0.54, p=$ 0.0004).

\section{Discussion}

In this study, we have demonstrated the potential of VOCs in exhaled breath analysis for diagnosis and monitoring of IBS patients. The first aim of our study was to apply a holistic metabolomics approach to identify biomarkers for IBS. We analysed a large number of exhaled VOCs and searched for differences in patterns between IBS patients and healthy controls. A pattern of 16 VOCs was identified to be most discriminative for the presence or absence of IBS in a large group of well phenotyped IBS patients and healthy controls (MIBS cohort), with a sensitivity of $89.4 \%$ and specificity of $73.3 \%$. Furthermore, we have shown that the selected VOCs correlated with IBS symptoms and were not affected by other host or disease-related factors.

To our knowledge, this is the first time that a set of VOCs in exhaled air was able to predict the presence of a common functional gastrointestinal disorder, which can be considered an important first step forward in the design and development of reliable non-invasive biomarkers for IBS. The classification RF model and the selected discriminatory VOCs were validated twice. First, validation was a standard part of RF using the bootstrap aggregating approach. The second validation consisted of an independent validation set of IBS patients and healthy controls not used in any steps of model optimization and creation. This procedure ensures the reliability of the classification RF model and the correct selection of the set of discriminatory VOCs. 
As the diagnosis of IBS is based on symptoms, our second aim was to test whether our VOC biomarker set would also correlate with severity of GI symptoms in these subjects. Four $\mathrm{Gl}$ symptom scores showed a moderate though highly significant correlation ( $r=0.55, p=0.0003)$ with the set of 16 VOCs in the clinical IBS cohort. This finding could be clinically useful, with the prospect to improve monitoring of IBS disease course in intervention studies, especially given the high placebo response in these patients.

Since functional GI symptoms observed in IBS patients are common in the general population, next we tested a possible application of the VOCs in a general population cohort (LL DEEP). This cohort was not included in the current study as an external validation cohort, but to test the potency of the biomarker panel to pick up the presence of gastro-intestinal symptoms in the general population. We used the same set of VOCs and tested the correlation to GI symptom scores in these study participants. The set of 16 VOCs also correlated moderately but significantly with $\mathrm{GI}$ symptom scores in this subpopulation $(r=0.54, p=0.0004)$. This finding is relevant for several reasons. First, it enables identifying subjects with $\mathrm{Gl}$ symptoms among the general population. Second, it may offer an opportunity to monitor effects of interventions in relation to underlying pathophysiological mechanisms. However, for the latter a longitudinal study design is needed to further test this hypothesis. Since the collection of exhaled breath is non-invasive, easy to apply, and requires only a minimal time investment, this method is potentially suitable for use in large cohorts and highly prevalent conditions in the general population.

Although each VOC can be interpreted and analysed separately, it is the combination of the 16 selected VOCs that yielded the highest discriminatory power between IBS patients and healthy controls. The biological interpretation and background of the compounds is difficult because of the complexity of the underlying metabolic pathways. However, even without the mechanisms being fully understood and explored, our VOC-biomarker set has potential value for diagnostics. IBS, or at least a considerable subgroup of the IBS population, is characterized by low grade 
chronic inflammation or immune activation [25], which results in increased oxidative stress leading to oxidation of larger molecules including lipids (e.g., polyunsaturated fatty acids) [26]. This may give degradation products like hydrocarbons, dienes, aldehydes, lipid peroxides and isoprostanes, which are excreted as VOCs, and subtle changes may be detected in breath. Indeed, the majority of our selected VOCs belong to the (saturated or unsaturated) hydrocarbon family. Some of them, like $n$ hexane, n-heptane and 1,4-cyclohexadiene, are increased in the IBS group, while others, like butane and 2-undecane, are increased in healthy controls. These compounds were all identified earlier as potential oxidative stress markers [27-29].

Another factor known to contribute to IBS is changes in an altered gut microbial composition [30]. Products of microbial origin are found in exhaled breath, indicating that systemic alterations of the gut flora may also be one of the factors influencing breath composition [31,32]. Interestingly, aziridine was found to be increased in breath of IBS patients in this study. This is a parent compound for organic substances containing the aziridine functional group, three-membered heterocyclic containing nitrogen. Aziridine containing compounds were previously detected in faeces but have not been described in exhaled breath before [33]. Aziridine containing compounds are isolated from the soil bacteria, Streptomyces caespitosus and lavendualeare, which are capable of living in symbiosis with plants, fungi, and animals [34]. Since they belong to the Actinobacteria Phyla, one of the four predominant phyla in the human body, it can be speculated that aziridines may be produced by this or other similar types of gut bacteria [35]. Antibiotic use is more frequent among IBS patients and certain microbiota strains may overgrow in a postantibiotic, replenished gut [36], leading to different metabolic breath profiles containing for instance, aziridine. It has to be noted that in our study population none of the IBS patients used antibiotics at least two weeks prior to inclusion. Aziridine origin in VOCs is purely speculative, nevertheless it suggests interesting connections that should be investigated further.

Some limitations of our study should be discussed. One may argue that a diagnosis of IBS is based purely on symptoms and thus the need for biomarkers in IBS is 
limited. However, daily practice is quite different, revealing high health care costs for diagnostic processing in IBS. In addition to the Rome III criteria reliable biomarkers could facilitate the diagnostic process of IBS [37] or may aid in identifying relevant subgroups for targeted therapeutic interventions [5]. We initiated this study as a first step using a metabolomics approach to explore IBS patients can be differentiated from controls whether based on an exhaled metabolite profile. We acknowledge that further validation is needed to replicate our data and to test the potency of our VOCs biomarker set in a second independent cohort in discriminating between IBS patients and subjects with comparable Gl symptoms based on organic causes, such as Inflammatory Bowel Disease or Coeliac Disease. Furthermore, in depth research is needed to investigate the origin of the discriminatory VOCs and their potential link to the pathophysiology of IBS.

\section{Conclusions}

A set of 16 VOCs in exhaled breath can be used to distinguish IBS patients from healthy controls with $89.4 \%$ sensitivity and $73.3 \%$ specificity. We also demonstrated a moderate but significant correlation between sets of GI symptoms and the VOCsbiomarker set for both clinical IBS patients and subjects in a general population cohort. This is the first study that shows the relevance of VOCs in exhaled air for the diagnostic process and for monitoring therapeutic interventions in IBS patients, but also demonstrates its potential to be used as a monitoring tool for gastrointestinal symptoms in the general public. 


\section{References:}

1. Canavan, C., J. West, and T. Card, The epidemiology of irritable bowel syndrome. Clin Epidemiol, 2014. 6: p. 71-80.

2. Chey, W.D., J. Kurlander, and S. Eswaran, Irritable bowel syndrome: a clinical review. JAMA, 2015. 313(9): p. 949-58.

3. Longstreth, G.F., et al., Functional bowel disorders. Gastroenterology, 2006. 130(5): p. 148091.

4. Plavsic, I., et al., Diagnosis of Irritable Bowel Syndrome: Role of Potential Biomarkers. Gastroenterol Res Pract, 2015. 2015: p. 490183.

5. Camilleri, M., Review article: biomarkers and personalised therapy in functional lower gastrointestinal disorders. Aliment Pharmacol Ther, 2015. 42(7): p. 818-28.

6. Sood, R. and A.C. Ford, Diagnosing irritable bowel syndrome with a combination of biomarkers and "psychomarkers". Gastroenterology, 2014. 146(5): p. 1418-20.

7. de Boer, N.K.H., et al., The Scent of Colorectal Cancer: Detection by Volatile Organic Compound Analysis. Clinical Gastroenterology and Hepatology, 2014. 12(7): p. 1085-1089.

8. Khalid, T., P. Richardson, and C.S. Probert, The Liver Breath! Breath Volatile Organic Compounds for the Diagnosis of Liver Disease. Clinical Gastroenterology and Hepatology, 2014. 12(3): p. 524-526.

9. Bodelier, A.G.L., et al., Volatile organic compounds in exhaled air as novel marker for disease activity in Crohn's disease; a metabolomic approach. Inflammatory Bowel Diseases, 2015. in press.

10. Kurada, S., et al., Review article: breath analysis in inflammatory bowel diseases. Aliment Pharmacol Ther, 2015. 41(4): p. 329-41.

11. Arasaradnam, R.P., et al., Review article: next generation diagnostic modalities in gastroenterology--gas phase volatile compound biomarker detection. Aliment Pharmacol Ther, 2014. 39(8): p. 780-9.

12. Patel, N., et al., Metabolomic analysis of breath volatile organic compounds reveals unique breathprints in children with inflammatory bowel disease: a pilot study. Aliment Pharmacol Ther, 2014. 40(5): p. 498-507.

13. Mujagic, Z., et al., Small intestinal permeability is increased in diarrhoea predominant IBS, while alterations in gastroduodenal permeability in all IBS subtypes are largely attributable to confounders. Aliment Pharmacol Ther, 2014. 40(3): p. 288-97.

14. Stolk, R.P., et al., Universal risk factors for multifactorial diseases: LifeLines: a threegeneration population-based study. Eur J Epidemiol, 2008. 23(1): p. 67-74.

15. Tigchelaar, E.F., et al., Cohort profile: LifeLines DEEP, a prospective, general population cohort study in the northern Netherlands: study design and baseline characteristics. BMJ Open, 2015. 5(8): p. e006772.

16. Dallinga, J.W., A. Smolinska, and F.J. van Schooten, Analysis of volatile organic compounds in exhaled breath by gas chromatography-mass spectrometry combined with chemometric analysis. Methods Mol Biol, 2014. 1198: p. 251-63.

17. Smolinska, A., et al., Current breathomics--a review on data pre-processing techniques and machine learning in metabolomics breath analysis. J Breath Res, 2014. 8(2): p. 027105.

18. Guyon, I. A scaling law for the Validation-set Training-set size ratio. 1997.

19. Snee, R.D., Validation of regression models: Methods and examples. Technometrics, 1977. 19(4): p. 415-428.

20. Breiman, L., Bagging predictors. Machine Learning, 1996. 24(2): p. 123-140.

21. Esbensen, K.H. and P. Geladi, Principles of Proper Validation: use and abuse of re-sampling for validation. Journal of Chemometrics, 2010. 24(3-4): p. 168-187.

22. Engel, J., et al., Regularized MANOVA (rMANOVA) in untargeted metabolomics. Analytica Chimica Acta, 2015. 899: p. 1-12.

23. Benjamini, Y. and Y. Hochberg, Controlling the False Discovery Rate - a Practical and Powerful Approach to Multiple Testing. Journal of the Royal Statistical Society Series BMethodological, 1995. 57(1): p. 289-300.

24. Hardoon, D.R., S. Szedmak, and J. Shawe-Taylor, Canonical correlation analysis: an overview with application to learning methods. Neural Comput, 2004. 16(12): p. 2639-64.

25. Ohman, L. and M. Simren, Pathogenesis of IBS: role of inflammation, immunity and neuroimmune interactions. Nat Rev Gastroenterol Hepatol, 2010. 7(3): p. 163-73. 
26. Mete, R., et al., The role of oxidants and reactive nitrogen species in irritable bowel syndrome: a potential etiological explanation. Med Sci Monit, 2013. 19: p. 762-6.

27. Phillips, M., et al., Detection of lung cancer with volatile markers in the breath. Chest, 2003. 123(6): p. 2115-23.

28. Poli, D., et al., Exhaled volatile organic compounds in patients with non-small cell lung cancer: cross sectional and nested short-term follow-up study. Respir Res, 2005. 6: p. 71.

29. Chen, X., et al., A study of the volatile organic compounds exhaled by lung cancer cells in vitro for breath diagnosis. Cancer, 2007. 110(4): p. 835-44.

30. Rajilic-Stojanovic, M., et al., Intestinal microbiota and diet in IBS: causes, consequences, or epiphenomena? Am J Gastroenterol, 2015. 110(2): p. 278-87.

31. Turner, C., P. Spanel, and D. Smith, A longitudinal study of ammonia, acetone and propanol in the exhaled breath of 30 subjects using selected ion flow tube mass spectrometry, SIFTMS. Physiol Meas, 2006. 27(4): p. 321-37.

32. Lindinger, W., et al., Endogenous production of methanol after the consumption of fruit. Alcohol Clin Exp Res, 1997. 21(5): p. 939-43.

33. de Lacy Costello, B., et al., A review of the volatiles from the healthy human body. J Breath Res, 2014. 8(1): p. 014001.

34. Seipke, R.F., M. Kaltenpoth, and M.I. Hutchings, Streptomyces as symbionts: an emerging and widespread theme? Fems Microbiology Reviews, 2012. 36(4): p. 862-876.

35. Johnson, C.L. and J. Versalovic, The human microbiome and its potential importance to pediatrics. Pediatrics, 2012. 129(5): p. 950-60.

36. Mendall, M.A. and D. Kumar, Antibiotic use, childhood affluence and irritable bowel syndrome (IBS). European Journal of Gastroenterology \& Hepatology, 1998. 10(1): p. 59-62.

37. Sood, R., et al., Systematic review with meta-analysis: the accuracy of diagnosing irritable bowel syndrome with symptoms, biomarkers and/or psychological markers. Aliment Pharmacol Ther, 2015. 42(5): p. 491-503. 


\section{Abstract}

Background: Disappearance of macroscopic mucosal inflammation predicts longterm outcome in Crohn's disease (CD). It can be assessed by ileocolonoscopy, which is, however, an invasive and expensive procedure. Disease activity indices do not correlate well with endoscopic activity and non-invasive markers have a low sensitivity in subgroups of patients. Volatile organic compounds (VOCs) in breath are of increasing interest as non-invasive markers. The aim of the present study was to investigate whether VOCs can accurately differentiate between active CD and remission.

Methods: Patients participated in a 1-year follow-up study and Harvey Bradshaw index $(\mathrm{HBI})$, blood, fecal and breath samples were collected at regular intervals. Patients were divided in 2 groups: active (Fecal calprotectin (FC) $>250 \mu \mathrm{g} / \mathrm{g}$ ) or inactive $(\mathrm{HBI}<4$ and $\mathrm{CRP}<5 \mathrm{mg} / \mathrm{l}$ and $\mathrm{FC}<100 \mu \mathrm{g} / \mathrm{g})$ disease. Breath samples were analyzed by gas chromatography-time-of-flight mass spectrometry. Random Forest analyses were used to find the most discriminatory VOCs.

Results: 835 breath grams were measured; 140 samples were assigned as active 135 as inactive disease and 110 samples of healthy controls. A set of 10 discriminatory VOCs correctly predicted active CD in $81.5 \%$ and remission in $86.4 \%$ (sensitivity 0.81 , specificity 0.80, AUC 0.80 ). These VOCs were combined into a single disease activity score which classified disease activity in more than $60 \%$ of the previously undetermined individuals.

Conclusions: We showed that VOCs can separate healthy controls and patients with active $C D$ and $C D$ in remission in a real-life cohort. Analysis of exhaled air is an interesting new non-invasive application for monitoring mucosal inflammation in inflammatory bowel disease. 


\section{Introduction}

Crohn's disease (CD) is a chronic relapsing inflammatory disease of the gastrointestinal tract and with ulcerative colitis (UC) referred to as inflammatory bowel disease (IBD) [1]. Chronic mucosal inflammation in CD will eventually lead to irreversible bowel damage with complications like strictures or fistula. Adequate monitoring of (sub)clinical mucosal inflammation is therefore of major importance in optimizing therapeutic strategies and preventing complications. Ileocolonoscopy remains the gold standard for assessment of macroscopic mucosal inflammation but is an expensive and invasive procedure. Disease activity indices (e.g., Crohn's Disease Activity Index (CDAI)) have been developed for systematic evaluation of the outcome in clinical trials [2]. However, the indices are cumbersome in clinical practice and potentially overlap with symptoms of irritable bowel syndrome or noninflammatory strictures. Furthermore, recent studies show that CDAl is not a reliable predictor of endoscopic disease activity [3, 4].

Several non-invasive biomarkers are used in clinical practice, measuring an increased concentration of proteins in serum and stool [5]. C-reactive protein (CRP) and Erythrocyte sedimentation rate (ESR) reflect systemic inflammation and are not specific for CD. Furthermore, CRP and ESR response are not found in all IBD patients with active inflammation [6-9]

Fecal calprotectin (FC) shows good diagnostic precision in distinguishing IBD from healthy controls [10]. FC seems a reliable predictor of relapse in IBD and correlates significantly with endoscopic disease activity [11-14]. Although FC is a sensitive marker for colonic inflammation, it is not a specific marker for IBD because elevated levels are also found in patients with infections, neoplasia or after use of nonsteroidal anti-inflammatory drugs [13]. FC is probably less sensitive for inflammation of the proximal colon or small bowel [15-17]. Besides, fecal consistency can influence $\mathrm{FC}$ values significantly in consecutive fecal samples. Storage temperature is also essential as $\mathrm{FC}$ values can drop at room temperature but remain stable for weeks when stored at $-20^{\circ} \mathrm{C}$ [18]. Moreover, FC values in the "grey zone" between 100 and $250 \mu \mathrm{g} / \mathrm{g}$, probably reflecting low inflammatory activity in the gut, are hard 
to classify.

Recently there is an increasing interest in the analysis of exhaled air due to great potential in clinical diagnostics, the potential to develop at home testing and the noninvasive nature. Besides detection of various inflammatory diseases of the lung and liver [19-21], some small studies indicate that exhaled air can also be used for gastrointestinal disorders. In 1994, Sedghi et al., studied 17 UC patients and showed that tissue damage in UC could be detected by reactive oxygen species (ROS)induced lipid peroxidation products like ethane [22]. Furthermore, Pelli et al., measured breath alkanes by gas chromatography in 10 active UC and 10 active CD patients and found significant differences in ethane, propane, and pentane between IBD patients and controls, but not for butane and isoprene [23]. These studies proved that excessive lipid peroxidation is an important pathophysiologic factor in IBD and can non-invasively be assessed. These initial studies only focused on a limited number of compounds from a specific pathophysiologic pathway. However, many metabolic processes together with a changed microbiome play a role in IBD and may result in alterations of exhaled VOCs [24, 25]. Therefore, assumption-less evaluation of all the excreted VOCs in exhaled air, would probably lead to a better selection of biomarkers that are able to reflect the complex metabolic processes in the bowel wall during active inflammation in IBD [26, 27]. Over the past years we have developed and applied an efficient technology platform by using exhaled air as a matrix to measure large numbers of VOCs and to select those relevant for disease [28].

We hypothesize that VOC profiles clearly differ between healthy controls, people with active $C D$ and inactive $C D$ and therefore the aim of our present study is to investigate whether our non-invasive $\mathrm{VOC}$ monitoring tool can accurately differentiate between $C D$ patients in remission or with active disease in a real-life cohort. 


\section{Materials and Methods}

\section{Patient inclusion}

Between October 2009 and December 2010, consecutive CD patients visiting the outpatient clinic of the Maastricht University Medical Center (MUMC) were included in a 1-year prospective follow-up study. The study was approved by the Medical Ethics Committee of the MUMC. Written informed consent was obtained from all patients. All patients had an established diagnosis of CD based on clinical, endoscopic, histological and/or radiological criteria. Demographic and clinical data (including phenotype according to Montreal classification, history of bowel resection) were obtained using the computer-based registration database.

Patients were assessed at study entry, at regular ( 3 monthly) visits or when a flare occurred. At each visit clinical activity scores, blood and fecal samples, and breath samples were collected. An endoscopy or radiological evaluation was performed whenever necessary for routine care. CRP, ESR and FC were analyzed routinely by the laboratory of Clinical Chemistry.

The group of healthy controls was recruited at the MUMC between 2009 and 2012 as part of the Maastricht cohort (NCT00775060) and partially at the department of gastroenterology. For each individual brief medical history was taken to eliminate the possibility of gastrointestinal disorders.

The Harvey Bradshaw index ( $\mathrm{HBI}$ ) was used for clinical assessment of disease activity (cut-off point for remission $\leq 4$ points). However, to define active disease or remission as accurate as possible in the absence of endoscopic evaluation, we used the following definitions: $\mathrm{FC}>250 \mu \mathrm{g} / \mathrm{g}$ was considered as active disease, the combination of $\mathrm{HBI}<4$ points and $\mathrm{CRP}<5 \mathrm{mg} / \mathrm{l}$ and $\mathrm{FC}<100 \mu \mathrm{g} / \mathrm{g}$ as remission. The patients with clearly defined disease activity based on fecal/serum markers and clinical activity index were used in the primary statistical analysis to build the classification model. The patients who could not be clearly diagnosed as having active disease or remission were included in the secondary endpoint statistical analysis. 
We have chosen not to perform routine endoscopic evaluation as this is not feasible in a real-life cohort. However, both FC $>250 \mu \mathrm{g} / \mathrm{g}$ and elevated CRP have shown correlation with endoscopic inflammation in $C D$ and by combining several markers endoscopic disease activity is probably most closely reflected [4, 12, 29].

\section{Sampling and analysis of exhaled air}

For collection of exhaled air each subject was asked to inflate $5 \mathrm{~L}$ bags (Tedlar Bag, SKC Ltd, Dorset, UK). One hour before sampling, eating, smoking and exercise were not allowed. To avoid the possible effect of diurnal variation of VOCs in breath the individuals were sampled randomly over the entire day. All patient samples were collected in the same room in order to prevent the appearance of a background bias. The bag contents were transferred to carbon-filled stainless steel sorption tubes (Markes International, Llantrisant Business Park, UK) where the VOCs were trapped within 2 hours after collection. Until analysis the air-tight capped tubes were stored at room temperature. The exhaled air samples were measured by thermal desorption-gas chromatography combined with time-of-flight mass spectrometry (GC-tof-MS) as previously described [28]. The experimental settings for GC and tofMS can be found elsewhere.[30] The compounds after separation by GC were identified by tof-MS (Thermo Electron Tempus Plus time-of-flight mass spectrometer, Thermo Electron Corporation, Waltham, USA). Detailed description of compounds identification via tof-MS is included in Supplementary Materials.

\section{Data handling}

\section{Statistical analysis via Random Forest}

Data pre-processing of the raw data is implemented before the actual statistical analysis, as previously described [31]. Shortly, the data preprocessing starts with removing the beginning and end of each chromatogram (retention time in minutes either $<1.3$ or $>23 \mathrm{~min}$ ) because of noisy mass spectra and column bleeding. In the next step noise removal and baseline correction were applied via wavelets [32] and P-splines [33], respectively. Subsequently, the chromatographic drifting due to column ageing, temperature differences and different sample compositions was 
corrected by adjusting retention times of all samples by Correlation Optimized Warping [34]. For each chromatogram peak picking was performed by finding all local maxima and corresponding minima. Finally, peaks found in chromatograms for each sample were merged by combining the corresponding compounds based on retention time and similarities in mass spectra. To make the chromatograms comparable, the final step of preprocessing involved probabilistic quotient normalization (PQN) [35]. In PQN the values of each observation are divided by the constant that accounts for the size effect due to variations sample volume and/or sample concentration. The final outcome is a data matrix containing relative abundances of measured VOCs.

Figure 1 shows the flowchart for statistical analysis. The first step consisted of selecting from all available data those breath-o-grams that were defined as active and inactive $C D$ patients according to the definition explained in the methods section. In addition, healthy controls were included. In step 2 the multivariate statistical method Random Forest (RF) [36] was used to find discriminatory VOCs profile. The data matrix containing the relative VOCs abundances was used in RF analysis. For that purpose, 3 different discriminatory RF models were built (each based on 1000 trees), using training data (containing $80 \%$ of samples of each group) selected using Duplex method [37]. First RF algorithm was applied on VOCs from healthy controls and $C D$ patients in remission to find compounds linked to remission. The second classification model was constructed on breath-o-grams from healthy controls and active CD patients for selection of VOCs related to active CD. The third model was applied on VOCs from patients in active or inactive CD. The performance of each $\mathrm{RF}$ model is shown using the receiver operating characteristic curve (ROC). The input to ROC analysis consisted of scores obtained from RF analysis. These scores are group (e.g., remission or active) probabilities. The scores for each sample add up by definition to 1 (e.g., 0.8 for remission and 0.2 for active). The scores can be considered as a range of cut-off for which individual sensitivities and specificities are calculated and represented in the ROC curve. The optimal cut-off is used (here 0.5) to obtain the sensitivity and specificity of the classification RF model. The sensitivity was calculated as the ratio between true positive and sum of true positive and false 
negative. Specificity was calculated as ratio between true negative and sum of true negative and false positive. In step 3 the compounds selected as significant in step 2 are joined and the final RF model is constructed using breath-o-grams from healthy controls, active and remission CD (step 4). To demonstrate differences between groups Principal Component Analysis (PCA) [38] is performed on proximity matrix obtained from the final RF model (step 5). Proximity matrix contains a similarity score between different breath-o-grams. The more similar two breath-o-grams are the higher the similarity score is. PCA allows visualizing the relation between all breatho-grams. In the resulting figure, score plot, each breath-o-gram is represented as a point and shows how all breath-o-grams are related to each other. Hence, the points close to each other have similar VOCs profile, while objects that lie far away are characterized by different properties. It is important to note that PCA belongs to the family of unsupervised techniques. However, here it is applied on proximity matrix obtained from RF, which is a supervised method.

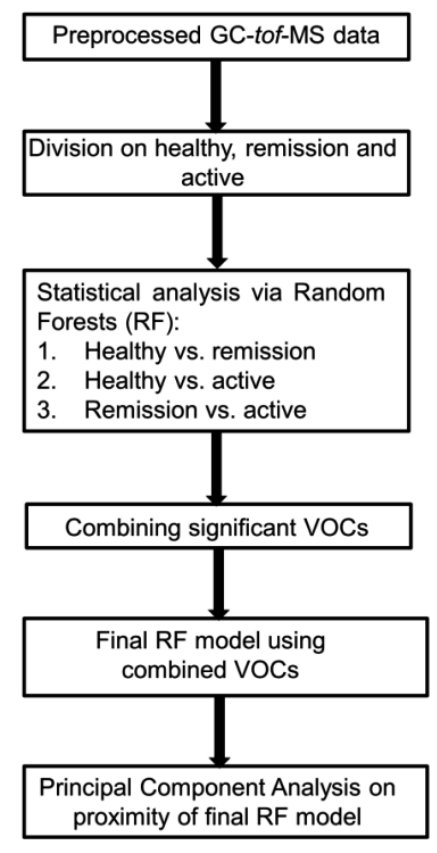

Data after preprocessing

Data containing healthy individuals, real active and real remission CD patient (step 1)

Selection of significant VOCs for three different comparisons (step 2)

Joining the set of significant VOCs (step 3)

Final discrimination model between healthy, remission and active (step 4)

Visualization of final distinction between healthy, individuals, remission and active $\mathrm{CD}$ patients using a set of significant VOCs (step 5)

Figure 1: The conceptual flow chart of statistical analysis 
The outcomes of the study were validated twice. The first validation is a standard part of RF methodology, where the so called out-of-bag classification error is used to justify the accuracy of the statistical model. For the second validation we used an independent test set of patients (containing approximately $20 \%$ of samples of each group) that was not used in the optimization and training (i.e., selecting discriminatory VOCs profile) of the RF discriminatory models.[39] In case of the first RF model (healthy controls vs. remission) the validation set consisted of the 20 breath-o-grams obtained from controls and 45 samples from the remission group. The training set contained 90 samples from healthy controls and CD remission. In the RF model of healthy controls versus active $C D$, the training set consisted of 90 samples from each group. This model was tested with a validation set of 20 healthy controls and 50 samples of active CD. In the final RF model, the validation set consisted of 27 breath-o-grams of patients with active disease and 22 in remission. The remaining 113 measurements were used as training set.

In the current study we aim for a robust marker that is able to discriminate active from inactive disease regardless of differences in disease location, medication, intoxication, smoking and diet. These effects were tested by PCA performed on proximity matrix as well as via Friedman statistical test. In case of medication the effect of four frequently used drugs in IBD were examined: budesonide/ prednisone, azathioprine, mesalazine, adalimumab and infliximab. In our cohort no information was available on food intake. The effect of diet was, thus, checked using another cohort of 1307 individuals (LifeLines Deep Cohort [40]), where food questionnaires were available.

In our pilot study, $80 \%$ of the active CD patients were correctly classified. Thus, using a power of 0.80 and an expected effect size of 0.5 , a group size of 150 is required to observe differences between the groups with a $95 \%$ confidence level.

\section{Defining disease activity score via VOCs}

Using RF analysis, the most discriminatory compounds were selected. The information enclosed in the discriminatory compounds was combined into a single value. RF analysis allows assigning for each selected compound an importance 
indicating the contribution of the compound in the discrimination of the studied groups. Using these importance values, each grown tree in RF analysis delivers the response (i.e., active as 1 or remission as 0 group) for each sample and a single score (here called disease activity score) for each available sample. This score summarizes all relevant information regarding disease activity and represents the probability of active disease or disease in remission. To obtain the response, the data go through each decision tree in RF analysis, starting from the beginning (so called root node) down to a leaf node, at each step an if/then rule is applied (e.g., if VOC1>10 go to left branch if not to right branch). The leaf node contains the obtained response and score. The final score is the average value among 1000 trees. This implies that if over 1000 trees an individual obtained 890 times response active (i.e., 1 ) and only 110 times a response remission (i.e., 0 ) the final activity score is 0.89 .

\section{Results}

\section{Patients and healthy controls}

In total, $191 \mathrm{CD}$ patients were included in the prospective follow-up study. Patient characteristics are listed in Table 1. During the 1-year follow-up period each CD patient collected 3-9 breath samples, in total 725 samples. At time of inclusion $26 \%$ of patients had active disease according to our definition of FC $>250 \mu \mathrm{g} / \mathrm{g}$. Median (IQR) values for HBI, CRP and FC were 3 (1-5), 4.8 (1.6-17.3) and 99.0 (22.0-272.0), respectively.

One hundred and ten gender and age matched healthy controls were included in the study. This cohort consisted of 62 females (54\%) and 48 males (46\%) with a mean age of $44.9 \pm 16.8$ years. Fourteen percent of the healthy controls were current smokers, $24 \%$ smoked in the past. In addition, $28 \%$ of CD patients were current smokers and $35 \%$ were ex-smokers. A brief medical history was taken in all healthy controls but revealed no underlying gastrointestinal disorders. FC was not determined in this population. 


\begin{tabular}{|c|c|}
\hline & $\mathrm{N}=191$ \\
\hline Female & $121(63 \%)$ \\
\hline Age (in years; mean $\pm S D$ ) & $43.6 \pm 14.8$ \\
\hline \multicolumn{2}{|l|}{ Smoking (\%) } \\
\hline current & 28 \\
\hline past & 35 \\
\hline never & 37 \\
\hline \multicolumn{2}{|l|}{ Fecal calprotectin $^{1}$} \\
\hline active disease & $502(336-891)$ \\
\hline remission & $15(14-43)$ \\
\hline \multicolumn{2}{|c|}{ Montreal classification (\%) } \\
\hline \multicolumn{2}{|c|}{ Age at diagnosis } \\
\hline A1<16y & 7 \\
\hline A2 17-40y & 64 \\
\hline A3 $\geq 41 y$ & 29 \\
\hline \multicolumn{2}{|c|}{ Disease location } \\
\hline L1 ileal & 27 \\
\hline L2 colonic & 22 \\
\hline L3 ileocolonic & 46 \\
\hline L4 proximal & 5 \\
\hline \multicolumn{2}{|c|}{ Disease phenotype } \\
\hline B1 non-structuring/nonpenetrating & 70 \\
\hline B2 structuring & 20 \\
\hline B3 penetrating & $13^{2}$ \\
\hline Surgery & $113(59 \%)$ \\
\hline ileocecal resection & $58(30 \%)$ \\
\hline (partial) colectomy & $29(15 \%)$ \\
\hline
\end{tabular}

Table 1: Patient characteristics and disease phenotype according to the Montreal classification. ${ }^{1}$ Fecal calprotectin in $\mu \mathrm{g} / \mathrm{g}$, values are represented as medians (Interquartile range) ${ }^{2} 3 \%$ of patients with penetrating disease also had structuring disease 


\section{Statistical analysis}

Data

In total 835 GC-tof-MS breath-o-grams were measured (725 CD patients and 110 healthy controls) of which 9 from CD patients were removed due to poor quality of the recorded chromatograms. The final 826 breath-o-grams contained 3813 VOCs. From these 3813 VOCs, most had almost only zero values. Therefore, a compound is only kept for analysis if it can be detected in at least $20 \%$ of samples in one of the experimental groups [41]. The final data matrix contained 487 VOCs. The dataset used for statistical analysis contained 110 breath-o-grams of healthy controls, 135 of CD patients in remission and 140 of active CD patients. Disease activity could not be determined according to our predefined criteria based on $\mathrm{HBI}, \mathrm{CRP}$ and FC in the remaining 441 samples.

\section{Random Forests analysis}

The first RF model was implemented on breath-o-grams of healthy controls and CD remission from training set. ROC analysis done on out-of-bag cases showed a sensitivity of 0.96 , a specificity of 0.99 and an area under the curve (AUC) of 0.99 for the diagnosis of CD in remission. The corresponding ROC curve for out-of-bag cases is shown in Figure 2A. The validation of this RF model with an independent test set gave an overall correct prediction of healthy controls or CD remission of $92.3 \%$. This model was based on a VOCs profile of 6 discriminatory compounds. A heat map with identification of the compounds is shown in Figure 3A. The relative concentrations of isoprene, acetone, and the unidentified compound (VOC1 retention time $(R T)=12.2$ minutes $(\mathrm{min})$ were found to be lower in the CD remission group compared to healthy controls, while heptadecane, undecanal and one unidentified compound (VOC2 RT = 10.7min) were higher. 

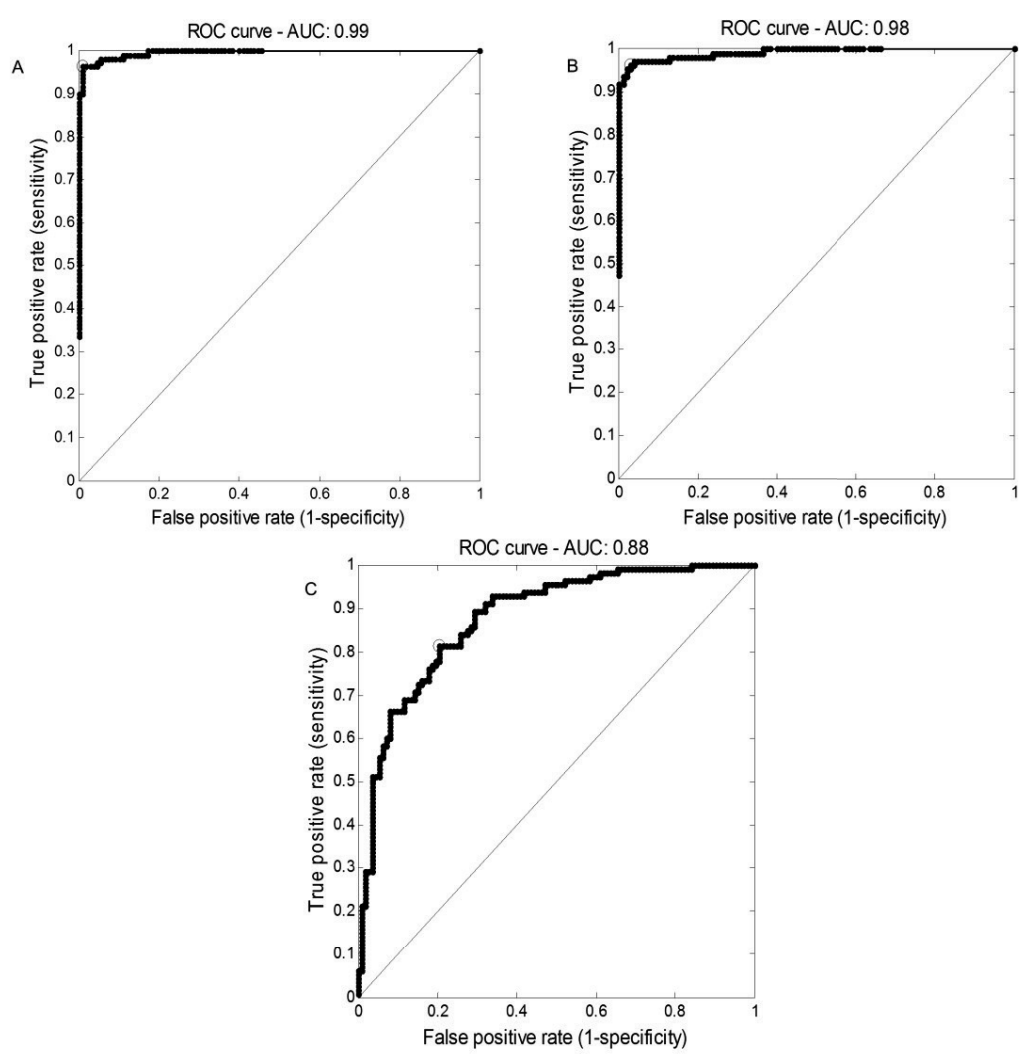

Figure 2: ROC curves for out-of-bag cases: (A) RF model for healthy controls and $C D$ remission using 6 VOCs. AUC is 0.99; (B) RF model for healthy controls and active CD using 7 VOCs. AUC is 0.98; (C) RF model for $C D$ remission and active $C D$ using 10 VOCs. $A \cup C$ is 0.88 .

The ROC curve of the second RF analysis (healthy controls vs active $C D$ ) is shown in Figure 2B with a sensitivity of 0.96 , specificity of 0.97 and AUC of 0.98 for the diagnosis of active $\mathrm{CD}$. The overall correct prediction for the independent test set was $97.3 \%$. This model yielded VOCs profile of 7 discriminatory compounds (Figure $3 B)$. The relative concentrations of unidentified compound VOC1 (RT = 12.2 $\mathrm{min}$ ), isoprene and acetone were decreased in the active $C D$ group, while those of 2,2,4trimethylpentane, branched saturated C12 compound (RT = 16.6min), 1-butoxy-2propanol and heptadecane, are elevated. 


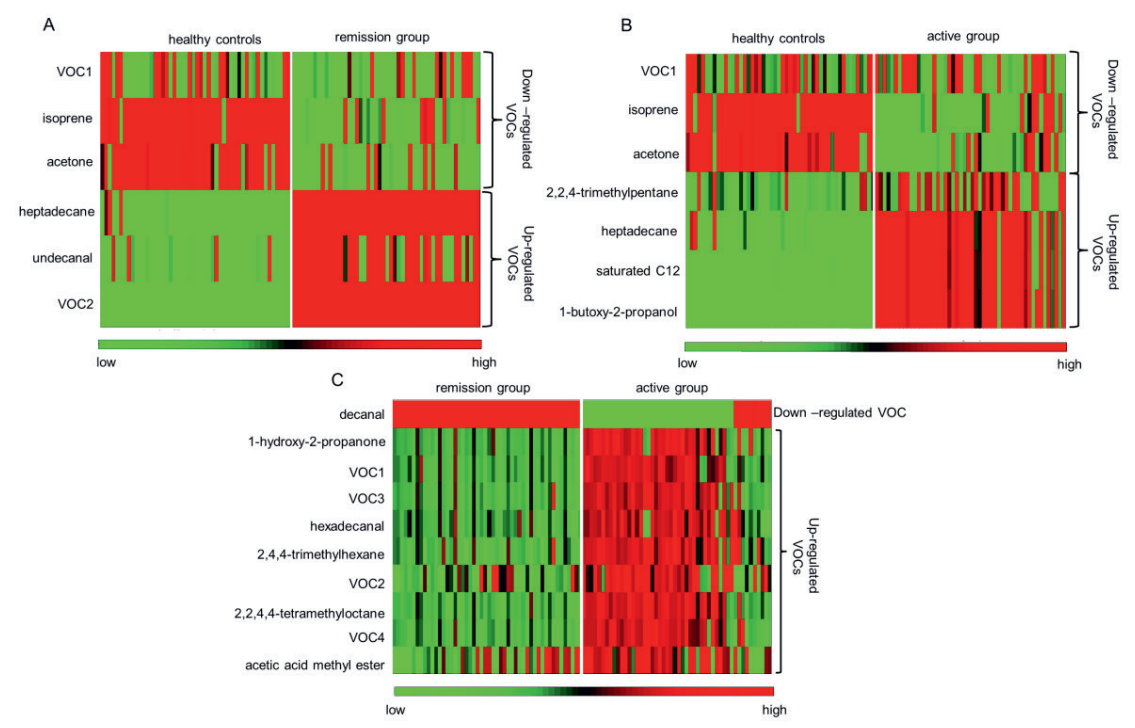

Figure 3: Heat maps of discriminatory VOCs: (A) RF analysis of healthy controls and CD remission. The relative concentrations of unknown compound (VOC1 RT=12.2min), isoprene and acetone are lower in $C D$ remission group compared to healthy controls, levels of heptadecane, undecanal and unknown compound (VOC2 RT=10.7min) are higher; (B) RF analysis of healthy controls and active CD. Relative concentrations of unknown compound (VOC1 $R T=12.2 \mathrm{~min})$ isoprene and acetone are lower in active $C D$ group in comparison to healthy controls, relative concentrations of 2,2,4-trimethylpentane, heptadecane, branched saturated $C 12$ compound and 1-butoxy-2-propanol are higher; (C) RF analysis of CD remission and active $C D$. Only the relative concentration of decanal is lower in the active $C D$ group compared to $C D$ in remission. The concentrations of remaining 9 VOCs (acetic acid, 1-hydroxy-2-propanone, 2,4,4trimethylhexane, 2,2,4,4-tetramethyloctane, hexadecanal and 4 unknown compounds VOC1 $R T=12.2 \mathrm{~min}, V O C 2 R T=10.7 \mathrm{~min}, \mathrm{VOC} 3 R T=20.6 \mathrm{~min}$ and VOC4 $R T=23.8 \mathrm{~min}$ ) are higher in active $C D$.

The last statistical RF model was built with breath-o-grams from patients with active and inactive $\mathrm{CD}$. ROC analysis performed on out-of-bag cases revealed a sensitivity of 0.81 , specificity of 0.80 and AUC of 0.88 (Figure $2 \mathrm{C}$ ). The validation of this RF model with an independent test set gave correct prediction of $81.5 \%$ of having active disease and $86.4 \%$ for remission. This model selected a profile of 10 discriminatory VOCs, represented in Figure 3C. From this discriminatory profile only the relative 
level of decanal was reduced in active $C D$ compared to $C D$ remission. The relative concentrations of the remaining $9 \mathrm{VOCs}$ (including unknown VOC1 $=12.2 \mathrm{~min}, \mathrm{VOC} 2$ $R T=10.7 \mathrm{~min}$, VOC3 RT= 20.6 min and VOC4 RT $=23.8 \mathrm{~min}$ ) were elevated in active $\mathrm{CD}$. Boxplots of discriminatory VOCs profile over time in 4 patients are shown in the Supplementary Materials (Figure S1). The variation of VOCs profile was comparable within each individual. However, more variations in VOCs profile were observed in an individual when disease activity changed over time. To further demonstrate the power of the set of 10 discriminatory VOCs to differentiate between active and inactive $C D$, a single disease activity score was obtained for validation set. The values of the single disease activity score were found to be significantly different between active $C D$ and $C D$ in remission (Friedman test $p<0.001$ )

PCA analysis demonstrated no groupings due to disease location, medication, smoking and intoxications. Moreover, Friedman test showed no significant changes in selected VOCs with respect to disease location, medication, smoking and intoxication. Based on the LifeLines Deep Cohort we could not find any relation between our discriminatory VOCs and diet.

The pairwise correlation between 10 discriminatory VOCs and FC level did not reveal any statistically significant relation. The moderate linear relation of 0.35 and $p$-value equal to 0.028 between the set of 10 VOCs and FC was found via Canonical Correlation Analysis.[42]

The last steps of statistical analysis (Figure 1, step 3-4) were to combine and implement the discriminatory profile of 17 VOCs in a final RF model. PCA performed on a proximity matrix from this RF model resulted in the score plot shown in Figure 4. Healthy controls, active $C D$ and $C D$ in remission patients were adequately separated with best separation between healthy controls and active CD. The fractional overlap between the groups of active $C D$ and remission indicates small similarities in VOC profiles. Nevertheless, this set of 17 VOCs allows discrimination of these 3 groups with high accuracy of $86.7 \%$. Note that the healthy controls display less variation than individuals with active $C D$ or remission, visualized in Figure 5 , where points corresponding to healthy controls (black stars) are very compact, while 
points related to $C D$ remission (red squares) and active $C D$ (blue circles) disease are spread more.

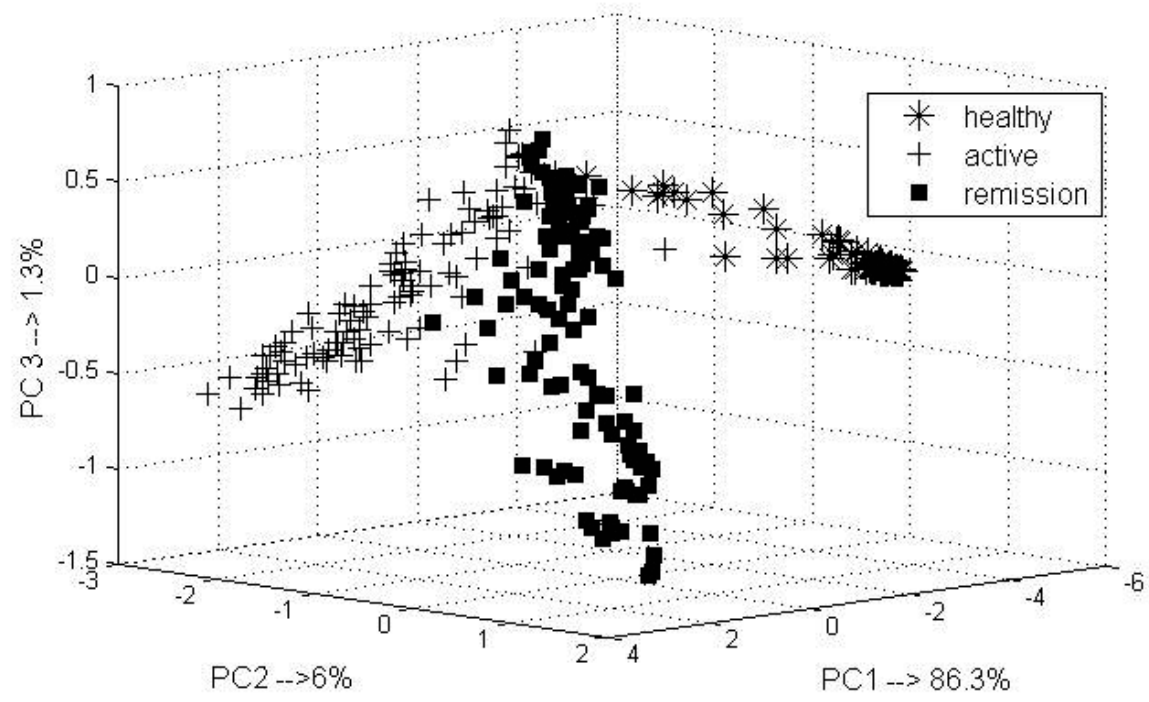

Figure 4: PCA score plot of proximity matrix from final RF model on breathograms from healthy controls, active $C D$ and $C D$ in remission using 17 discriminatory VOCs. Each individual is represented as a point (stars for healthy controls, squares for $C D$ in remission and circles for active $C D$ ). The three groups create distinct clusters indicating differences in VOCs profile.

VOCs as disease activity marker

As mentioned previously 716 high quality breath-o-grams were obtained from CD patients. For the statistical analysis only breath-o-grams corresponding to patients with clearly defined disease activity were used (135 inactive and 140 active samples). For the remaining samples of 164 patients, a disease activity score between 0 and 1 was calculated for each breath sample using the 10 discriminatory VOCs. Values close to 0 indicate remission while values close to 1 correspond with active disease. A score of approximately 0.5 indicates that disease activity cannot be defined. Using 3 clinical parameters namely FC, CRP and HBI more than $60 \%$ of patients remained with undefined disease activity, i.e., did not fulfill our strict criteria for active disease $(\mathrm{FC}>250 \mu \mathrm{g} / \mathrm{g}$ ) or remission $(\mathrm{HBI}<4$ points and $\mathrm{CRP}<5 \mathrm{mg} / \mathrm{l}$ and 
FC $<100 \mu \mathrm{g} / \mathrm{g}$ ). Using VOCs profile with a cut-off value of 0.7 for active and 0.3 for inactive disease only for $22 \%$ of samples disease activity could not be classified.

\section{Discussion}

We conducted a large prospective trial to use VOCs in exhaled air as a novel biomarker for disease activity in CD. The study showed that VOCs breath profiles are significantly different between healthy controls and patients with active $C D$ or $C D$ in remission. A profile of 10 VOCs differentiated active from inactive CD with a high sensitivity and specificity and an overall accuracy of $82-86 \%$ in an independent test set.

Lechner et al., [43] demonstrated the feasibility of exhaled air as novel diagnostic tool in the differential diagnosis of gastro-intestinal diseases but used a limited number of IBD patients $(n=10)$ and no identification of the significant compounds was performed. In a recent study on VOC analysis in children with IBD, patients and healthy controls could be classified with high accuracy by 3 discriminatory VOCs, but interestingly no correlation was found with degree of inflammation [44]. Since repeated evaluation of mucosal inflammation in $C D$ is crucial for clinical decision making, a marker for assessment of disease activity in patient follow-up is needed. Therefore, the current study focuses specifically on disease activity in CD using a large cohort of $191 \mathrm{CD}$ patients. To our knowledge this is the first large prospective study evaluating the role of VOCs profiles in exhaled air in relation to disease activity of CD patients. We used a metabolomic approach to separate patients with active and inactive $C D$ and developed a VOC disease activity marker based on the 10 discriminatory VOCs. This VOC activity marker was significantly higher in active CD patients and was able to classify patients in the "grey zone" of disease activity according to the definition of disease activity based on HBI, CRP and FC. However, it should be mentioned that the VOC activity marker was not validated in an independent cohort of patients with endoscopic disease activity as gold standard. This study proves that VOCs profiling in exhaled air is a promising new way of monitoring disease activity in CD. 
VOC analysis of exhaled air is increasingly recognized as diagnostic tool for detection of diseases [45]. Several studies have been performed on detection of inflammatory disease of the lung and liver using VOCs in breath [20, 21, 46], and very recently also studies on detection of gastrointestinal cancer have been published. In a study on colorectal cancer a set of 15 VOCs was able to detect colorectal cancer in 62 patients versus 41 controls with an overall accuracy of $76 \%$. Levels of some specific VOCs were higher in colorectal cancer patients as other VOCs showed more variable profiles suggesting that it is the pattern rather than a single $\mathrm{VOC}$ to be representative for the metabolic changes in colorectal cancer [24, 47]. In our study 9 VOCs showed higher concentrations in the active CD group compared to only $1 \mathrm{VOC}$ with higher concentration in the remission group. In addition, acetone and isoprene were 2 of the discriminating compounds in our study on healthy controls versus active $\mathrm{CD}$ or $\mathrm{CD}$ in remission. In the study by Altomare et al., on colorectal cancer both compounds played no role in discrimination of patients and controls. Most discriminatory compounds in their study were mainly end products of lipid peroxidation like alkanes, alkenes and aldehydes [24]. Acetone and isoprene are one of the most abundant VOCs in human breath and the pathophysiological process of their formation is discussed below. A recent paper showed that it is also possible to detect colorectal cancer and advanced adenomas by an electronic nose recognizing VOC profiles in fecal gas. Sensitivity and specificity for discriminating healthy controls from colorectal cancer patients were 85 and $87 \%$ respectively [25]. However, analysis of fecal gas or exhaled air by an electronic nose does not allow chemical identification of individual components.

Several discriminatory VOCs we found in this study (e.g., enhanced alkanes and methylated alkanes, aldehydes) are probably derived from lipid peroxidation of polyunsaturated fatty acids found in cellular membranes. Several studies show that oxygen mediated injury trough increased free radical production and impaired antioxidant defense systems play an important role in the pathophysiology of IBD $[48,49]$. Hydrocarbons as the end products of lipid peroxidation show low solubility in blood and are quickly excreted into breath after formation and can be used to monitor the degree of oxidative damage [26]. Another discriminatory VOCs acetone 
is formed by the process of lipolysis and produced by hepatocytes via decarboxylation of excess acetyl-CoA generated from fatty acid $\beta$-oxidation [50]. One of the most common VOCs in exhaled air is isoprene which is formed along the mevalonic pathway of cholesterol synthesis and has been identified as marker of disorders in the cholesterol metabolism like familial hypercholesterolemia but also in patients with peptic ulcer disease or gastric cancer [47]. Interestingly, in our study isoprene level was decreased in patients with active and quiescent $C D$ in comparison to healthy controls. This may be due to some impairment of membrane repair mechanisms in epithelial cells. The same mechanism was already reported in inflammatory lung diseases and in patients with chronic heart failure [51, 52]. Another explanation may be connected to lipid metabolic changes. It has been shown that a decrease of isoprene level in exhaled air may be related to activation of the immune system [53].

Since the microbiome is different in IBD patients compared to healthy persons, the change in VOC profiles could also be the result of alterations in the gut microbiome. The composition of VOCs in feces was found to be largely shared by healthy persons, but significantly different in patients with UC, Clostridium difficile or Campylobacter jejuni infection [54]. Moreover, a recent study of Ahmed et al. [55] showed clear differences of VOCs in feces of active IBD patients and patients with Irritable Bowel Syndrome (IBS). The main compounds responsible for the difference were several esters of short fatty acids and aldehydes. Similarly, we found altered level of acetic acid methyl ester in active CD. It has been shown [55] that unbalanced organic acids are related to changes in Lactobacilli and Veillonella, which are responsible for production and transformation of organic acids. Another group of compounds changed in active $C D$ is of the oxygen containing aldehydes, which are related to inflammation. One of the sources of aldehydes in the human body is the reduction of hydroperoxide by cytochrome p450 as a secondary product of lipid peroxidation [56]. Recently also detection and analysis of VOCs in urine have become of interest. Besides in breath and feces, VOCs can be detected in urine by several analytical methods like electronic nose technology and Field Asymmetric lon Mobility Spectrometer (FAIMS). In a study of 48 IBD patients and 14 controls, with 
both techniques patients and controls could be classified with accuracy of more than $75 \%$ [57]. Also, colorectal cancer can be distinguished from healthy controls by analysis of headspace urinary samples with FAIMS. Reclassification of colorectal cancer samples was correct in $74 \%$ of cases in a recent study in 83 patients and 50 healthy controls [58]. All these data on VOCs in breath, feces and urine support a pathophysiological rationale for the difference in VOCs profiles between active and inactive $C D$ reported in our study.

Finally, strengths and potential limitations of the methodology should be addressed. The strength of our statistical approach lies in reproduction in an independent test set. Such testing creates a higher level of validation and therefore gives stronger certainty to the findings. Moreover, the statistical methodology applied here is robust to outliers, i.e., patients different from the majority. This implies that the findings are representative for the majority of $C D$ patients and thus are not negatively affected by a single patient. The statistical model built here can be fast and easily applied to new cohorts and makes our approach generalizable. A possible limitation might be the sample size. Although the number of patients in our study is relatively large, the findings should be studied and validated in a larger multi center cohort with endoscopy as gold standard for evaluation of disease activity. Furthermore, a large number of VOCs is included in the study and we cannot rule out the possibility of contamination with VOCs from the environment. In the current study no ambient air was collected which might be considered as limitation of this study. However, all breath samples were taken at the same location (outpatient clinic). Since samples of individuals were taken alternately, the variation in ambient air affected all groups in a similar manner. Besides, the robust statistical approach filters out all the nondiscriminatory VOCs. We were not able to identify some of the discriminating compounds and therefore they can't be related to a certain pathophysiological process.

A possible limitation is that we did not use endoscopic assessment of disease activity as this is not feasible for repeated follow-up in a real-life cohort. However, by using FC (and a combination of $\mathrm{HBI}$ and $\mathrm{CRP}$ ) as increasingly accepted surrogate marker for endoscopic disease activity, this in our opinion should not have influenced the 
positive results of our study $[4,12]$. We did not use endoscopy as primary tool for evaluation of disease activity since we aimed to develop a monitoring tool that performs well in a real-life outpatient IBD population in daily clinical practice. In the current study only CD patients were assessed. Future studies should also focus on VOC analysis in UC patients, not only for monitoring of clinical disease activity, but also for differentiation between UC activity and the possibility of concomitant colorectal cancer, as symptoms between both conditions have significant overlap. The set of discriminatory VOCs was also investigated with respect to smoking because more CD patients were smokers or ex-smokers ( $68 \%$ versus $38 \%$ of healthy controls). However, we could not find any relation between the discriminatory VOCs and smoking behavior.

We conclude that VOCs can be used to discriminate CD from healthy controls and between active $C D$ and $C D$ in remission in a real-life cohort. The discriminatory VOCs, combined into a single disease activity score, enabled classifying patients that could not be classified with a combination of fecal and serological markers. Although, the study requires further validation in a new multicenter cohort with endoscopic assessment of inflammation as gold standard, it demonstrates the feasibility of exhaled air in monitoring disease activity in CD. Such study is the significant step towards development of point-of-care devices for assessing disease activity. We think that analysis of exhaled air is an interesting, new, and non-invasive application for monitoring IBD patients with potential to develop a home test. 


\section{References:}

1. Baumgart, D.C. and W.J. Sandborn, Crohn's disease. Lancet, 2012. 380(9853): p. 1590-605.

2. Best, W.R., et al., Development of a Crohn's disease activity index. National Cooperative Crohn's Disease Study. Gastroenterology, 1976. 70(3): p. 439-44.

3. Regueiro, M., et al., Crohn's disease activity index does not correlate with endoscopic recurrence one year after ileocolonic resection. Inflamm Bowel Dis, 2011. 17(1): p. 118-26.

4. af Bjorkesten, C.G., et al., Surrogate markers and clinical indices, alone or combined, as indicators for endoscopic remission in anti-TNF-treated luminal Crohn's disease. Scand J Gastroenterol, 2012. 47(5): p. 528-37.

5. Vermeire, S., Review article: genetic susceptibility and application of genetic testing in clinical management of inflammatory bowel disease. Alimentary Pharmacology \& Therapeutics, 2006. 24: p. 2-10.

6. Fagan, E.A., et al., Serum levels of C-reactive protein in Crohn's disease and ulcerative colitis. Eur J Clin Invest, 1982. 12(4): p. 351-9.

7. Solem, C.A., et al., Correlation of C-reactive protein with clinical, endoscopic, histologic, and radiographic activity in inflammatory bowel disease. Inflamm Bowel Dis, 2005. 11(8): p. 70712.

8. Henriksen, M., et al., C-reactive protein: a predictive factor and marker of inflammation in inflammatory bowel disease. Results from a prospective population-based study. Gut, 2008. 57(11): p. 1518-23.

9. Vermeire, S., G. Van Assche, and P. Rutgeerts, Laboratory markers in IBD: useful, magic, or unnecessary toys? Gut, 2006. 55(3): p. 426-31.

10. von Roon, A.C., et al., Diagnostic precision of fecal calprotectin for inflammatory bowel disease and colorectal malignancy. Am J Gastroenterol, 2007. 102(4): p. 803-13.

11. Schoepfer, A.M., et al., Fecal calprotectin correlates more closely with the Simple Endoscopic Score for Crohn's disease (SES-CD) than CRP, blood leukocytes, and the CDAI. Am J Gastroenterol, 2010. 105(1): p. 162-9.

12. D'Haens, G., et al., Fecal calprotectin is a surrogate marker for endoscopic lesions in inflammatory bowel disease. Inflamm Bowel Dis, 2012. 18(12): p. 2218-24.

13. Tibble, J.A., et al., High prevalence of NSAID enteropathy as shown by a simple faecal test. Gut, 1999. 45(3): p. 362-6.

14. Tibble, J.A., et al., Surrogate markers of intestinal inflammation are predictive of relapse in patients with inflammatory bowel disease. Gastroenterology, 2000. 119(1): p. 15-22.

15. Garcia-Sanchez, V., et al., Does fecal calprotectin predict relapse in patients with Crohn's disease and ulcerative colitis? J Crohns Colitis, 2010. 4(2): p. 144-52.

16. D'Inca, R., et al., Can calprotectin predict relapse risk in inflammatory bowel disease? Am J Gastroenterol, 2008. 103(8): p. 2007-14.

17. Costa, F., et al., Calprotectin is a stronger predictive marker of relapse in ulcerative colitis than in Crohn's disease. Gut, 2005. 54(3): p. 364-8.

18. Dhaliwal, A., et al., Utility of faecal calprotectin in inflammatory bowel disease (IBD): what cutoffs should we apply? Frontline Gastroenterology, 2014.

19. Van Berkel, J.J., et al., A profile of volatile organic compounds in breath discriminates COPD patients from controls. Respir Med, 2010. 104(4): p. 557-63.

20. Verdam, F.J., et al., Non-alcoholic steatohepatitis: A non-invasive diagnosis by analysis of exhaled breath. Journal of hepatology, 2013. 58(3): p. 543-8.

21. Dallinga, J.W., et al., Volatile organic compounds in exhaled breath as a diagnostic tool for asthma in children. Clinical and Experimental Allergy, 2010. 40(1): p. 68-76.

22. Sedghi, S., et al., Elevated breath ethane levels in active ulcerative colitis: evidence for excessive lipid peroxidation. Am J Gastroenterol, 1994. 89(12): p. 2217-21.

23. Pelli, M.A., et al., Breath alkanes determination in ulcerative colitis and Crohn's disease. Dis Colon Rectum, 1999. 42(1): p. 71-6.

24. Altomare, D.F., et al., Exhaled volatile organic compounds identify patients with colorectal cancer. Br J Surg, 2013. 100(1): p. 144-50.

25. de Meij, T.G., et al., Electronic nose can discriminate colorectal carcinoma and advanced adenomas by fecal volatile biomarker analysis: proof of principle study. Int J Cancer, 2013.

26. Miekisch, W., J.K. Schubert, and G.F. Noeldge-Schomburg, Diagnostic potential of breath analysis--focus on volatile organic compounds. Clin Chim Acta, 2004. 347(1-2): p. 25-39.

27. Boots, A.W., et al., The versatile use of exhaled volatile organic compounds in human health 
28. and disease. J Breath Res, 2012. 6(2): p. 027108

Van Berkel, J.J.B.N., et al., Development of accurate classification method based on the analysis of volatile organic compounds from human exhaled air. Journal of Chromatography B-Analytical Technologies in the Biomedical and Life Sciences, 2008. 861(1): p. 101-107.

29. Peyrin-Biroulet, L., et al., Clinical disease activity, C-reactive protein normalisation and mucosal healing in Crohn's disease in the SONIC trial. Gut, 2014. 63(1): p. 88-95.

30. Smolinska, A., et al., Profiling of volatile organic compounds in exhaled breath as a strategy to find early predictive signatures of asthma in children. PLoS One, 2014. 9(4): p. e95668.

31. Smolinska, A., et al., Current breathomics-a review on data pre-processing techniques and machine learning in metabolomics breath analysis. Journal of Breath Research, 2014. 8(2).

32. Walczak, B., Wavelets in Chemistry. 2000, Amsterdam: Elsevier Science.

34. Statistical Science, 1996. 11: p. 89-121. wavelength chromatographic profiles for chemometric data analysis using correlation optimised warping. Journal of Chromatography A, 1998. 805(1-2): p. 17-35.

35. Dieterle, F., et al., Probabilistic quotient normalization as robust method to account for dilution of complex biological mixtures. Application in H-1 NMR metabonomics. Analytical Chemistry, 2006. 78(13): p. 4281-4290.

36. $\quad$ Breiman, L., Random forests. Machine Learning, 2001. 45(1): p. 5-32.

37. Snee, R.D., Validation of regression models: Methods and examples. Technometrics, 1977. 19(4): p. 415-428.

38. Geladi, P., et al., Principal Component Analysis of Multivariate Images. Chemometrics and Intelligent Laboratory Systems, 1989. 5(3): p. 209-220.

39. Burgard, D.R. and J.T. Kuznicki, Chemometrics: Chemical and Sensory Data, ed. C. Press. 2000, Boca Rota, Florida, USA.

40. Stolk, R.P., et al., Universal risk factors for multifactorial diseases - LifeLines: a threegeneration population-based study. European Journal of Epidemiology, 2008. 23(1): p. 67-74.

41. Baranska, A., et al., Profile of volatile organic compounds in exhaled breath changes as a result of gluten-free diet. Journal of Breath Research, 2013. 7(3).

42. Hardoon, D.R., S. Szedmak, and J. Shawe-Taylor, Canonical correlation analysis: An overview with application to learning methods. Neural Computation, 2004. 16(12): p. 26392664.

43. Lechner, A., et al., Headspace screening of fluid obtained from the gut during colonoscopy and breath analysis by proton transfer reaction-mass spectrometry: A novel approach in the diagnosis of gastro-intestinal diseases. International Journal of Mass Spectrometry, 2005. 243(2): p. 151-154.

44. Patel, N., et al., Metabolomic analysis of breath volatile organic compounds reveals unique breathprints in children with inflammatory bowel disease: a pilot study. Aliment Pharmacol Ther, 2014. 40(5): p. 498-507.

45. Arasaradnam, R.P., et al., Review article: next generation diagnostic modalities in gastroenterology - gas phase volatile compound biomarker detection. Aliment Pharmacol Ther, 2014. 39(8): p. 780-9.

46. Chen, X., et al., $A$ study of the volatile organic compounds exhaled by lung cancer cells in vitro for breath diagnosis. Cancer, 2007. 110(4): p. 835-44.

47. Xu, Z.Q., et al., A nanomaterial-based breath test for distinguishing gastric cancer from benign gastric conditions. Br J Cancer, 2013. 108(4): p. 941-50.

48. Torres, M.I. and A. Rios, Current view of the immunopathogenesis in inflammatory bowel disease and its implications for therapy. World J Gastroenterol, 2008. 14(13): p. 1972-80.

49. Rezaie, A., R.D. Parker, and M. Abdollahi, Oxidative stress and pathogenesis of inflammatory bowel disease: an epiphenomenon or the cause? Dig Dis Sci, 2007. 52(9): p. 2015-21.

50. Buszewski, B., et al., Human exhaled air analytics: biomarkers of diseases. Biomed Chromatogr, 2007. 21(6): p. 553-66.

51. Miekisch, W., J.K. Schubert, and G.F. Noeldge-Schomburg, Diagnostic potential of breath analysis--focus on volatile organic compounds. Clinica chimica acta; international journal of clinical chemistry, 2004. 347(1-2): p. 25-39.

52. McGrath, L.T., R. Patrick, and B. Silke, Breath isoprene in patients with heart failure. European journal of heart failure, 2001. 3(4): p. 423-7.

53. Fuchs, D., et al., Decline of exhaled isoprene in lung cancer patients correlates with immune activation. J Breath Res, 2012. 6(2): p. 027101. 
54. Garner, C.E., et al., Volatile organic compounds from feces and their potential for diagnosis of gastrointestinal disease. FASEB J, 2007. 21(8): p. 1675-88.

55. Ahmed, I., et al., An investigation of fecal volatile organic metabolites in irritable bowel syndrome. PloS one, 2013. 8(3): p. e58204.

56. Haick, H., et al., Assessment, origin, and implementation of breath volatile cancer markers. Chem Soc Rev, 2014. 43(5): p. 1423-49.

57. Arasaradnam, R.P., et al., A novel tool for noninvasive diagnosis and tracking of patients with inflammatory bowel disease. Inflamm Bowel Dis, 2013. 19(5): p. 999-1003.

58. Arasaradnam, R.P., et al., Detection of colorectal cancer (CRC) by urinary volatile organic compound analysis. PLoS One, 2014. 9(9): p. e108750. 


\section{Supplementary material}

\section{Chemical analysis and compounds identification}

To remove air and water vapor prior analysis sorption and tubes were purged with a steady Helium stream $(20 \mathrm{ml} / \mathrm{min})$ for 5 minutes. Then, the volatile compounds trapped on the sorption tubes were released under a flow of helium using automated thermal desorption. Next, the vaporous mixture was divided into two parts, $90 \%$ of the sample was recollected on an identical sample tube and $10 \%$ of the sample was transferred to an electrically cooled sorbent trap $\left(5^{\circ} \mathrm{C}\right)$. The recollection tubes are used in case of an obvious instrumental problems occurred during the chemical analysis. In the current study it was necessary for two measurements. The sample was focused via cold trap filled with a $30 \mathrm{~mm}$ bed of Tenax TA backed up by a 30 $\mathrm{mm}$ Carbograph 1TD bed. From cold-trap sample was injected into a GC (Thermo Fischer Scientific, Austin, USA, column: Restek RTX-5ms, 30mx0.25mm ID, coated with $1.0 \mu \mathrm{m} \mathrm{HP}-5$ phase). The temperature of the GC was programmed as follows: first $40^{\circ} \mathrm{C}$ for 5 minutes then rose by $10^{\circ} \mathrm{C}$ every minute until $270^{\circ} \mathrm{C}$ was reached. This temperature was maintained for 5 minutes. Within tof-MS part electron ionization at $70 \mathrm{eV}$ was used with $5 \mathrm{~Hz}$ scanning rate over a range of $\mathrm{m} / \mathrm{z} 35-350$. The tof-MS allows for compounds detection and identification based on their total mass spectrum. Tof-MS measures the time necessary to travel in an electric field from the ionization source to a detector plate. Because all ions have the same kinetic energy $(E=1 / 2 m v 2)$, ions with different mass-to-charge ratio $(\mathrm{m} / \mathrm{z}$ values) are separated in the flight tube based on their velocity. Note, that due to practical limitations, i.e., absence of standard reference materials for VOCs analysis in exhaled air, compounds are quantified using relative quantification, i.e., determining the response ratio between a VOC and other VOCs. The output of GC-tof-MS consists of two dimensions: retention time and $\mathrm{m} / \mathrm{z}$ value. Thus, for each measured compound in breath sample, retention time and mass spectrum are available. These two parameters are used in the chemical identifications of the VOCs. Spectrum recognition is done using the National Institute of Standards and Technology mass spectral library. A compound is identified by searching in a reference library compounds which have similar spectra to the measured spectrum. These 
compounds are arranged in order of similarity to the measured spectrum and a so called "hit list" is obtained. The final identification is performed by the manual evaluation of measured mass spectra and the reference spectra of the compounds with the highest similarity index from the "hit list", retention time information and spectrum interpretation by an experienced Mass-spectrometrist.

A)

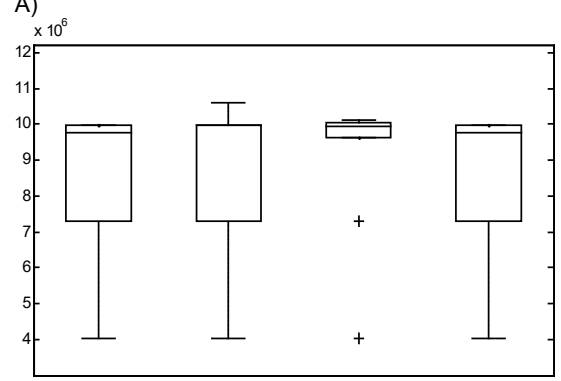

C) $\times 10^{6}$

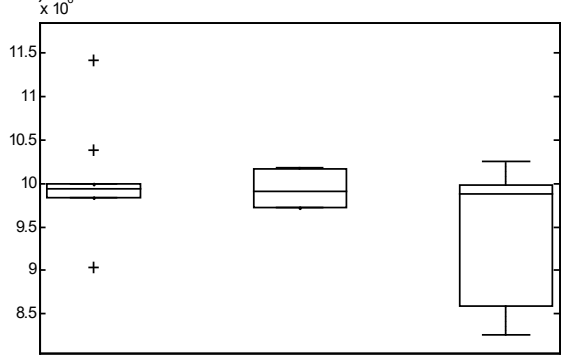

B)
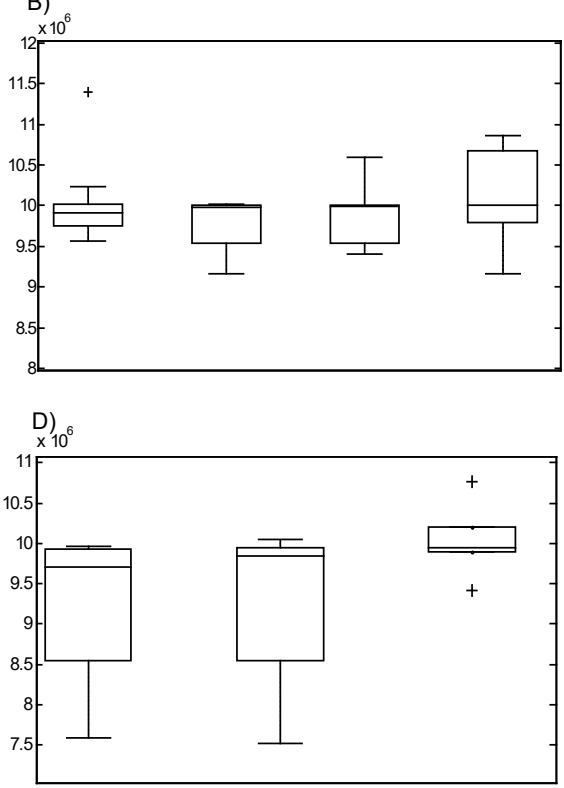

Figure S1. The boxplots of discriminatory VOCs profile over different visit for: A) a patient that over all visits stayed in remission; $B$ ) a patient that over all visits stayed in active stage of $C D$; $C$ ) a patient that over changed diseased stage from remission (visit 1) to active (visit 2 and 3); D) a patient that changed disease stage from remission (visit 1 and 2) to active (visit 3). The variation of VOCs profile usually was more diverse for patients changing the disease state over time. 


\section{Abstract}

Exhaled breath has proven to be a valuable source of information about humans' body. Subtle differences between volatile organic compounds (VOCs) formed endogenously can be detected and become a base for a potential monitoring tool for health and disease. Until now, there is a lack of biological and mechanistic knowledge of the processes involved in the production of relevant VOCs. Among the possible sources of health/disease related VOCs are microorganisms found in the respiratory tract and in the gut. Other VOCs in the body are produced by cells that are influenced by the disease, for instance due to metabolic disorders and/or inflammation. In order to gain insight in the in vivo production of VOCs by human cells and thus the exhaled breath composition, in vitro experiments involving relevant cells should be studied, as they may provide valuable information on the production of VOCs by the affected cells. To this aim we developed and validated a system for dynamically (continuously) collection of headspace air in vitro, using a Caco-2 cell line. The system allows the application of different cell lines, as well as different experimental setups including varying exposure times and treatment options while preserving cell viability. Significant correlation ( $p \leq 0.0001)$ between collection outputs within each studied group confirmed high reproducibility of the collection system. An example of such an application is presented here: we studied the influence of oxidative stress on the VOCs composition of the headspace air of Caco-2 cells.

By comparing the VOCs composition of air flushed through empty culture flasks $(n=35)$, flasks with culture medium $(n=35)$, flasks with medium and cells $(n=20)$, flasks with medium and an oxidative stressor $\left(\mathrm{H}_{2} \mathrm{O}_{2}\right)(\mathrm{n}=20)$, and flasks with medium, stressor and cells $(n=20)$, we were able to separate the effects from the stressor on the cells from all other interactions. Measurements were done with gas chromatography-time of flight-mass spectrometry. Multivariate data analysis allowed detection of significant altered compounds is compared groups.

We found a significant change $(p \leq 0.001)$ of the composition of VOCs due to the stressing of the Caco- 2 cells by $\mathrm{H}_{2} \mathrm{O}_{2}$. A total of 10 VOC's showed either increased 
or decreased abundance in the headspace of the cell cultures due to the presence of the $\mathrm{H}_{2} \mathrm{O}_{2}$ stressor.

\section{Introduction}

Exhaled breath has proven to be a valuable source of information about humans' body condition. Volatile organic compounds (VOCs) are produced by endogenous biochemical reactions connected to normal and disease-altered host metabolism and to different compositions of oral, lung and gut microbiota. Due to their low solubility in blood volatiles are transported from the place of origin, through the blood stream, to the lungs and excreted in breath, from which they can be captured and analysed. The composition of exhaled breath is further influenced by the inhaled breath composition, diet and by genotype and phenotype of the subjects. VOCs' profiles connected to disease or symptoms can be separated from irrelevant variations and confounders by either a strict and controlled sampling or by using proper and advanced statistical tools [1, 2]. Specific VOC profiles have already been correlated with the presence of chronic inflammatory pulmonary [3], intestinal $[4,5]$ and liver diseases [6-8], as well as with certain types of cancer [9-11]. So far, the chemical identification of many of the discriminating VOCs in such clinical studies has not led to a sufficient depth of their origin and clinical relevance understanding. In order to gain insight in the VOCs dynamics in vivo we developed an in vitro experimental culturing and sampling system to study the changes in VOC profiles due to changed VOC production by human cells. This system allows the investigation of the influence of certain exposures, treatments, and co-incubations on cell metabolism by monitoring the change in VOCs generation on a cellular level in a chosen in vivo situation.

The system was validated and the repeatability between subsequent collections was established. The potency of the system was demonstrated by an experiment in which we exposed Caco-2 cells to $\mathrm{H}_{2} \mathrm{O}_{2}$, a well-known oxidative stressor. The change in VOC profile due to the $\mathrm{H}_{2} \mathrm{O}_{2}$ was determined; the identity of 8 out of 10 significantly influenced VOCs could be established. 
Our choice of the Caco-2 cell line, a model of the intestinal barrier, is explained by our particular interest in breath analysis in relation to gastrointestinal health and disease. Several studies showed that increased oxidative stress and impaired antioxidant function play a role in a pathophysiology of IBD [12,13]. Recent work of our group has proven the value of the breath analysis in tracking disease activity in Crohn's disease [5].

\section{Materials and Methods}

\section{Sample collection system}

A schematic picture of our sampling equipment is shown in Scheme 1.

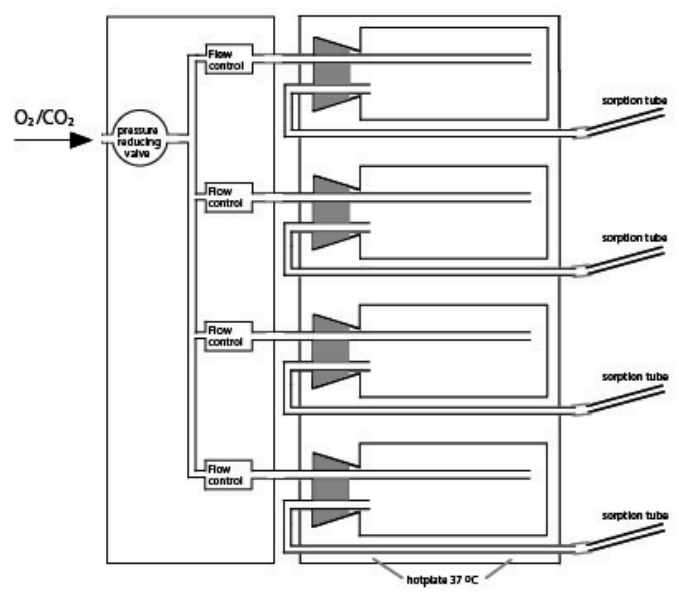

Scheme 1. System for collection of headspace air.

The system consists of a hot plate set at $37^{\circ} \mathrm{C}$. Four standard $\mathrm{T}-75 \mathrm{~cm}^{3}$ cell culture flasks (Corning ${ }^{\circledR}$ Flask, Corning Incorporate, NY, USA) are placed on top of this hot plate and can be simultaneously used for the collection of headspace samples. The culture flasks are covered with an isolating hood to maintain an evenly distributed, constant temperature of $37{ }^{\circ} \mathrm{C}$. Each flask cap has been replaced by a rubber cork with an inlet tube delivering a mixture of $95 \% \mathrm{O}_{2} / 5 \% \mathrm{CO}_{2}$ (carbogen) at a constant flow of $25 \mathrm{ml} / \mathrm{min}$ and an outlet tube connected to a stainless steel two-bed sorption 
tube, filled with carbograph 1TD/Carbopack X (Markes International, Llantrisant, Wales, UK) used for collection and storage of the excreted VOCs. After 1 hour of sampling, the tube is removed, end-capped and stored at room temperature until analysis.

\section{Samples}

To test and validate the system, the headspace air was collected for $1 \mathrm{~h}$ from 35 empty flasks (EF), 35 flasks containing only medium (MF) and 20 Flasks containing Caco-2 cells in medium (CF).

To show one of the possible applications of the system, headspace air was additionally collected from 20 flasks with $\mathrm{H}_{2} \mathrm{O}_{2}$ exposed Caco-2 cells in medium (ECF) and 20 Flask with $\mathrm{H}_{2} \mathrm{O}_{2}$ exposed medium control (EMF). This experimental set up allows the separation of the effect of $\mathrm{H}_{2} \mathrm{O}_{2}$ on Caco-2 cells from all other interactions.

\section{Cell culture}

Caco-2 cells ATCC (CRL-1593.2, Manasses, VA, USA) were cultured in $75 \mathrm{~cm}^{3}$ cell culture flasks (Corning ${ }^{\circledR}$ Flask, Corning Incorporate, NY, USA) with canted neck under standard conditions. We used Dulbecco's Modified Eagle Medium (DMEM) + $4.5 \mathrm{~g}$ glucose + L-glutamine + Sodium pyruvate) (no. 41966 Gibco, Invitrogen) with addition of $10 \%$ heat inactivated fetal calf serum (Gibco, Invitrogen), $1 \%$ of nonessential amino acids 100X (Sigma) and 1\% of penicillin-streptomycin 100X (Penicillin 5000 units/ml Streptomycin 5000 g/ml) (Gibco, Invitrogen).

\section{$\mathrm{H}_{2} \mathrm{O}_{2}$ exposure}

Cells were grown to $80-90 \%$ confluence in the culture flasks under standard culturing conditions using normal growth medium. Afterwards the standard growth medium was replaced by freshly prepared medium containing $100 \mu \mathrm{M} \mathrm{H} \mathrm{H}_{2} \mathrm{O}_{2}$ to induce oxidative stress [14]. 


\section{Cell viability}

To assure that the release or consumption of VOCs collected by the equipment is due to living cells and their natural metabolism, it is necessary to establish that the system does not cause substantial cell death. To this end, flasks with cells upon 80$90 \%$ confluence were transferred to the collection equipment located under the hood. Flasks caps were replaced with rubber cork and $1 \mathrm{~h}$ collection took place. Afterwards, cell viability was checked with the use of trypan blue staining in the cell counter (Countless TM Automated Cell Counter, Invitrogen).

\section{GC-MS analysis}

VOCs from the headspace air were trapped on the sorption tubes and measured by thermal desorption-gas chromatography combined with time-of-flight mass spectrometry (TD-GC-tof-MS) as previously described for exhaled breath measurements [15]. In short, volatiles were first released from the carbon packing of the tube using thermal desorption under a flow of helium. Then, the gaseous mixture was split, $90 \%$ of the sample was recollected onto an identical empty sorption tube whereas $10 \%$ was transferred to an electrically cooled sorbent trap $\left(5^{\circ} \mathrm{C}\right)$ from where it was injected into a GC (Thermo Fischer Scientific, Austin, USA. [Requirements and settings: column: Restek RTX-5ms, $30 \mathrm{~m} \times 0.25 \mathrm{~mm}$ ID, $1.0 \mu \mathrm{m}$ HP-5 phase; carrier gas: helium, flow rate: $1.5 \mathrm{~mL} / \mathrm{min}$; GC temp.: $40^{\circ} \mathrm{C} / 5 \mathrm{~min}$., $10^{\circ} \mathrm{C} / \mathrm{min}$ increase - until $\left.\left.270^{\circ} \mathrm{C}, 270^{\circ} \mathrm{C} / 5 \mathrm{~min}\right]\right)$. The components separated by GC were analyzed by time-offlight mass spectrometry (Tempus Plus, Thermo Fischer Scientific). Settings: lonization energy $70 \mathrm{eV}$, mass range $35-350 \mathrm{AMU}$, scanning rate: 5 scans/s.

\section{Data pre-processing}

Before actual data analysis, all raw chromatograms require steps dealing with variations in baseline and peak shift issues connected with column aging, complex matrix composition and temperature differences, as described previously [2]. In short: after removal of the first $1.3 \mathrm{~min}$ and the part exceeding 23 min of retention time $\left(\mathrm{t}_{\mathrm{r}}\right)$ for each chromatogram, noise removal took place by implying wavelets [16]. Chromatographic baseline correction was done via B-splines [17], whereas 
chromatographic drift was adjusted by $t_{r}$ of all samples by Correlation Optimized Warping [18]. Subsequently, chromatograms were merged by combining the corresponding compounds based on retention time and similarities in mass spectra. In the final step, probabilistic quotient normalization [19] was applied. This all allowed for the construction of one large data set with all samples and compounds present in these measurements.

\section{Data analysis}

Principle component analysis (PCA) was used to gain an overview of sample characteristics, trends in the data and presence of possible outliers. Partial Least Square Discriminant Analysis (PLS-DA) was performed to find VOCs that had significantly different abundances between studied groups. PLS-DA resembles PCA and is a latent variable method [20]. While in PCA the first principal component (PC) is constructed in the direction of the highest variance in the data, the latent variable (LV) of PLS will be built in the direction that explains the highest "between class" variation. Significant Multivariate Correlation (SMC) was used to statistically assess the importance of variables chosen by the PLS model, a method previously described by Tran at al. 2014[21] and thought to improve the evaluation of PLS originated important variables. Visualisation of the chosen subset of VOCs and its discriminative power is obtained by PCA analysis and results in a PCA score plot and/or PCA biplot. The PCA score plot shows a summary of all data points (chromatograms) and the relation between them. The closer points are to one another, the more similar profile they have.

Visualisation can be done with the use of biplot that combined information showed by traditional PCA score plot and relevant compounds' abundances. In a biplot, besides relations between groups also the relations between the measured VOCs are visualized. Here, the angle between compounds, plotted as lines, shows the correlation between them. The positive correlation is high when the angle between lines is small. An angle close to $90^{\circ}$ means there is no correlation between compounds, whereas angles close to $180^{\circ}$ show a strong negative correlation. Additionally, biplots allow the interpretation of the change of the relative 
concentration of compounds between groups. If the sign of PCs for sample and compound is the same (both positive or both negative) the concentration of those particular compounds are increased in these samples. Opposite signs point to a decrease of the compound.

\section{Study design}

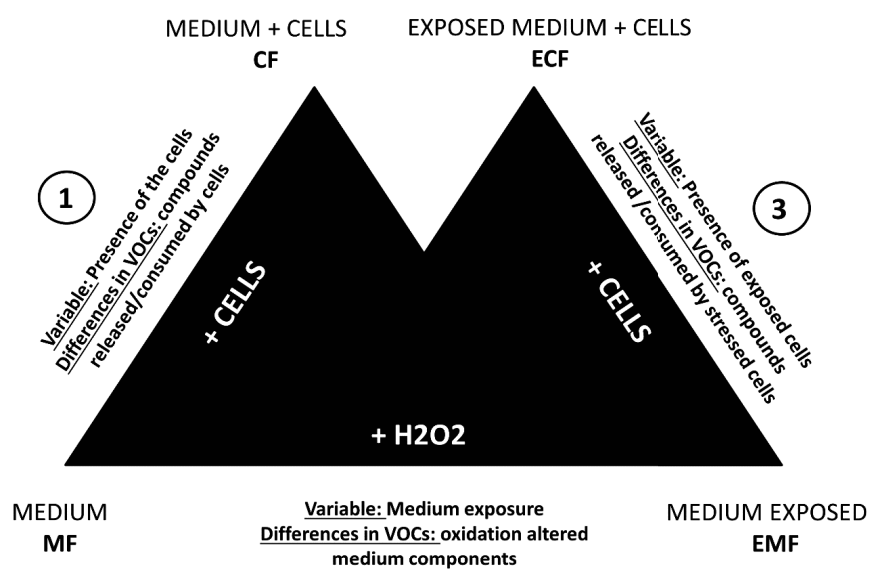

(2)

Scheme 2. Study design. Three comparisons of VOCs detected from headspace air of MF vs. CF (analysis no.1); MF vs. EMF (analysis no.2); EMF vs. ECF (analysis no.3).

Comparisons of VOCs released/consumed were performed for all samples combinations. As explained in Scheme 2, each comparison results in different output. VOCs significantly different between CF and MF that come up from analysis no. 1 represent compounds that are released/consumed by Caco- 2 cell line in normal condition. Analysis no.2 points to VOCs which are purely related to medium oxidation and not to presence of the cells whereas analysis no.3, looking at VOCs significantly different between ECF and EMF, point toward compounds released/consumed by stressed cells. Although it is analysis no. 3 that directly relates to the question about how oxidative stress influences VOCs released/consumed, all 
other comparisons need to be performed to exclude taking into account VOCs connected to normal cell metabolism and to products of medium oxidation.

\section{Compound identification}

Volatile compounds of interest were tentatively identified with the use of a NIST library spectrum recognition program, by further interpretation by an experienced mass spectrometry specialist and with the use of the retention time of the compounds.

\section{Results}

\section{Cell culture condition versus cell viability}

The mean viability of cells calculated from $20 \mathrm{CF}$ after $1 \mathrm{~h}$ collection time was $93.1 \%$ \pm 4.3 , indicating no significant cell death has occurred during sampling.

Similarly, viability was checked after headspace collection from flasks containing Caco- 2 cells exposed to $100 \mu \mathrm{M} \mathrm{H}_{2} \mathrm{O}_{2}$. The mean cell viability was $94.9 \% \pm 4.8$, thus was not affected by the hydrogen peroxide exposure.

\section{System reproducibility and variation between samples}

Headspace air from 35 empty flasks, 35 flasks with medium and 20 flasks with medium and cells was used to assess and determine the reproducibility of the collection system. Boxplots of the Pearson correlation (range 0-1) for samples collected from each group are visible in Figure 1. Each correlation was established by comparing the entire VOCs profile between samples in the respective groups. Standard deviations for the boxplots (I-III) were respectively: 0.039, 0.045, and 0.057 . 

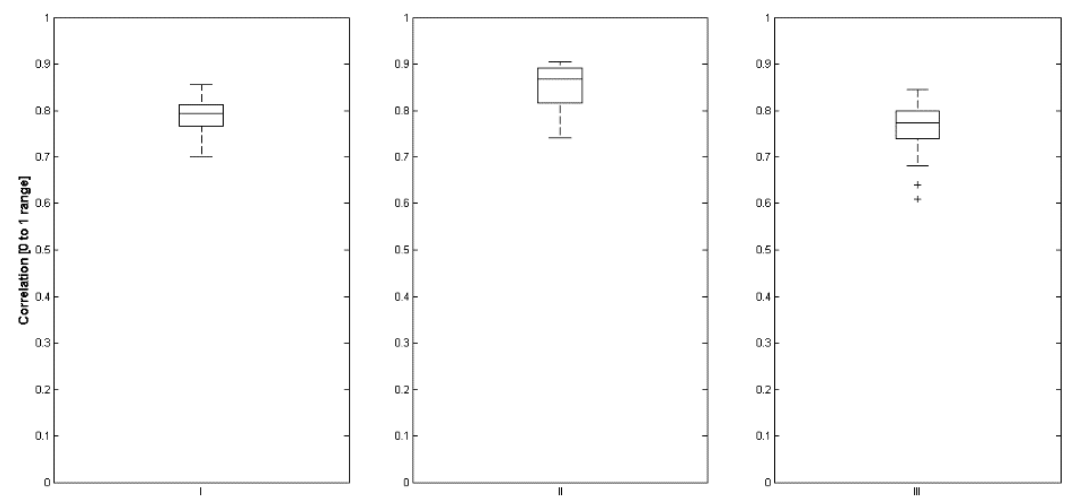

Figure 1. Correlation [range 0-1] between headspaces from I) 35 empty flasks (EF): Mean=0.79, II) 35 flasks with medium (MF): Mean=0.85, III) 20 flasks with medium and Caco-2 cells (CF): Mean=0.76. All correlations were statistically significant, $p$ value $\leq 0.0001$.

\section{Analysis}

The data matrix consisted of 130 samples (35 EF, 35 MF, 20 CF, 20 EMF, $20 \mathrm{ECF}$ ) and 3489 VOCs. Not all the compounds were present in all the measured samples, thus the original data consisted of many zero-values. This type of data contains a large amount of redundant information, which needs to be handled. Therefore, we applied the so-called $20 \%$ rule [22]: only components present in at least $20 \%$ of one of the groups were retained in the matrix. After applying the rule, we ended up with 686 VOCs in the data matrix.

As indicated in the analysis design (Scheme 2) three major comparisons were performed:

- analysis no.1: MF vs CF (normal cell metabolism),

- analysis no.2: MF/EMF (medium oxidation),

- analysis no.3: ECF/EMF (oxidative stress).

For each of these three comparisons in first instance PCA analysis was performed to see the overall similarities and differences between measured samples and to detect any outliers. A PCA score plot built upon all 686 compounds did not show clear samples grouping when performed for any of the 3 comparisons. Furthermore, 
Caco- 2 cells used for the experiments were utilized between passages 7-10 and no trends and groupings could be observed due to the different passage number, i.e., samples were randomly distributed within the score plots.

In the next step, we used a priori known structure of the data to find patterns in VOCs distribution related to specific groups. PLS-DA was used to find compounds with untargeted matter which were significantly different in headspace of MF/CF, $M F / E M F$ and ECF/EMF. The sMC was used to statistically assess the importance of VOCs chosen by each of the PLS-DA models. Using a significance level of $p \leq 0.001$, the SMC has chosen three sets of VOCs. A schematic overview of the number of compounds that arises from each comparison can be found in Scheme 3.

Out of 686 VOCs in the data matrix, 15 were significantly different $(p \leq 0.001)$ between medium only (MF) and medium + cells (CF) (analysis no.1). This difference can be connected to the Caco-2 cells and their normal metabolism. In analysis no.2, VOCs in the headspace of medium (MF) were compared to medium containing $100 \mu \mathrm{M}$ $\mathrm{H}_{2} \mathrm{O}_{2}$ (EMF). A total of 21 VOCs appeared to be significantly different between these groups, so these VOCs are connected to the oxidation of medium components. In analysis no. 3 the headspace of $\mathrm{H}_{2} \mathrm{O}_{2}$ exposed cells (ECF) is compared with the headspace of medium and $\mathrm{H}_{2} \mathrm{O}_{2}$ only (EMF). In this case 16 VOCs were detected to be significantly different between both groups. Similarly, to the first analysis, these two groups differ due to the presence of cells, in these case exposed ones. As can be seen from Scheme 3, only 10 VOCs out of the 16 VOCs seem to be exclusively related to the oxidation of the Caco-2 and their metabolism. This emphasizes the need of a proper study design. Due to controls, we can exclude 5 VOCs which repeatedly appeared significant in analysis no.2 having been related to medium oxidation and 1 volatile from analysis no. 1 related to normal cell metabolism. Further data analysis is focused on analyses no. 1 and 3 since these experiments concern normal and altered cell metabolism. 


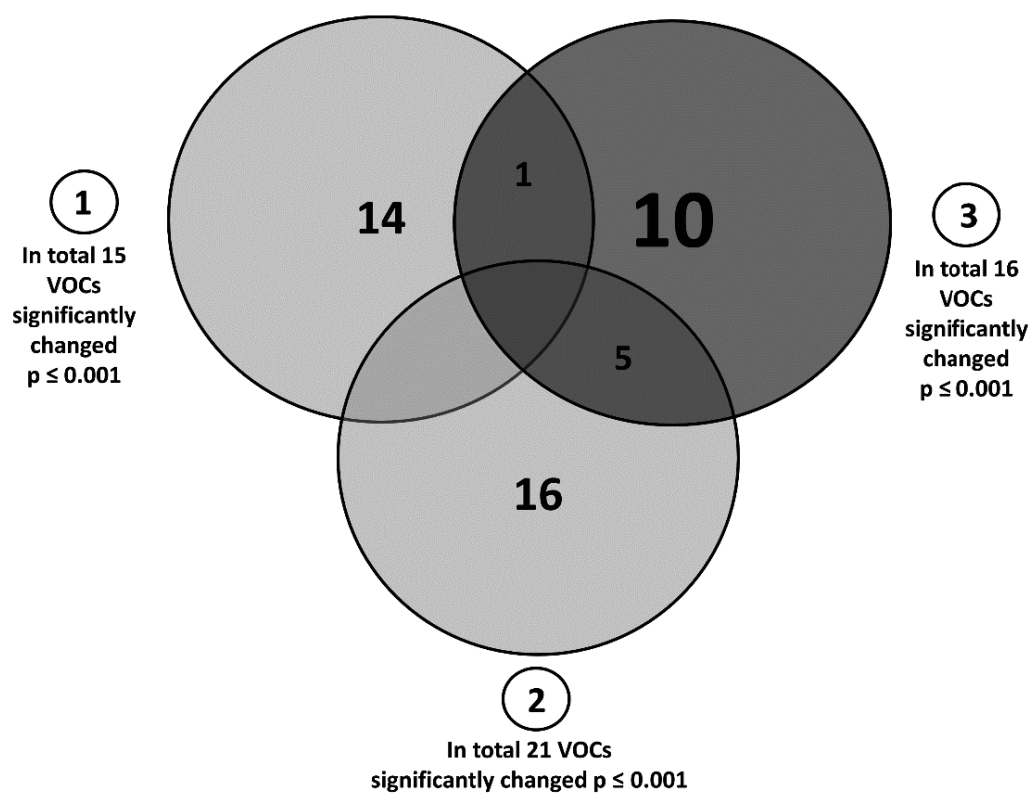

Scheme 3. Overview of the number of compounds that significantly changed in analyses no.1, 2 and 3 of analysis design Scheme 2.

\section{Analysis no. $1 \mathrm{MF} / \mathrm{CF}$}

To visualise the differences found in analysis no. 1, the PCA score plot was built on the relevant set of 15 VOCs (Figure 2). Out of these 15 compounds 8 could be tentatively identified. For the remaining 7 compounds identification was inconclusive. The trends in relative abundances, increase/decrease of the compounds, all 15 VOCs were considered. A group of 8 compounds including phenol was found in MF but not in CF. This means that the compounds should be derived from the medium but were entirely consumed by the cells in the CF group. Another group, consisting of 3 compounds including heptane was found in CF but not in MF, demonstrating its formation by the cells, while a third group, including 1-nonene or 2-nonene was present in both, MF and CF, but at different proportions. 


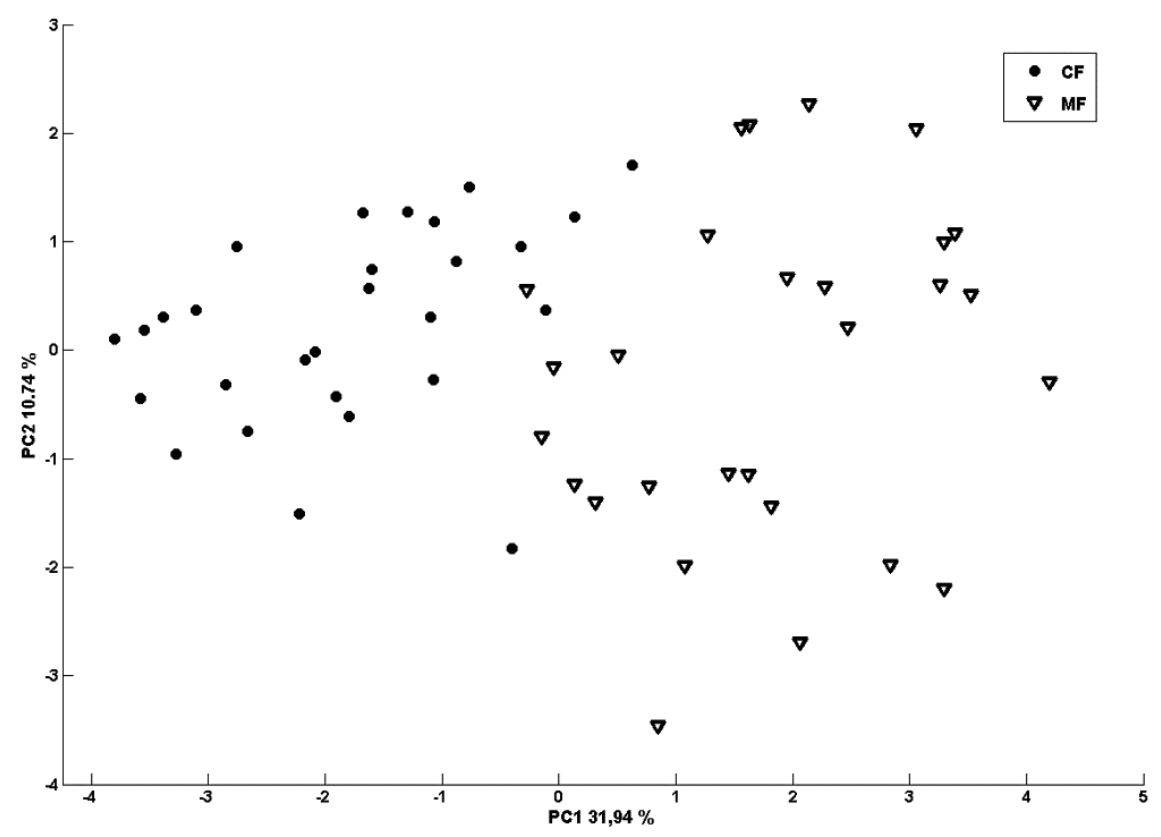

Figure 2. PCA Score plot obtained for 15 significant compounds selected by PLS-DA sMC. Dots represent CF (Caco-2 cultures), reverse triangles represent MF (Medium only). The groups are well separated along $P C 1$ indicating differences in the VOC profiles. From the plot it can be seen that the variation within both groups is rather similar.

\section{Analysis no. 3 EMF/ECF}

Here, the differences found in analysis no. 3 have been visualised in a biplot based on the PCA analysis of the relevant set of 10 VOCs (Figure 3). As can be seen from Figure 3, the separation between exposed Caco-2 samples, and exposed medium is reached along principal components 1 and 2 that explain over $60 \%$ of the total data variance.

Besides the separation between groups, Figure 3 also shows the changes in relative compounds concentrations indicated for each compound by dotted lines. As can be seen from the Figure, 4-methyl-2-pentene is clearly higher in the headspace of the exposed cells, whereas for instance 2-octene and the octadecane isomer $\mathrm{C}_{18} \mathrm{H}_{38}$ are higher in exposed medium only when compared to exposed cells. 


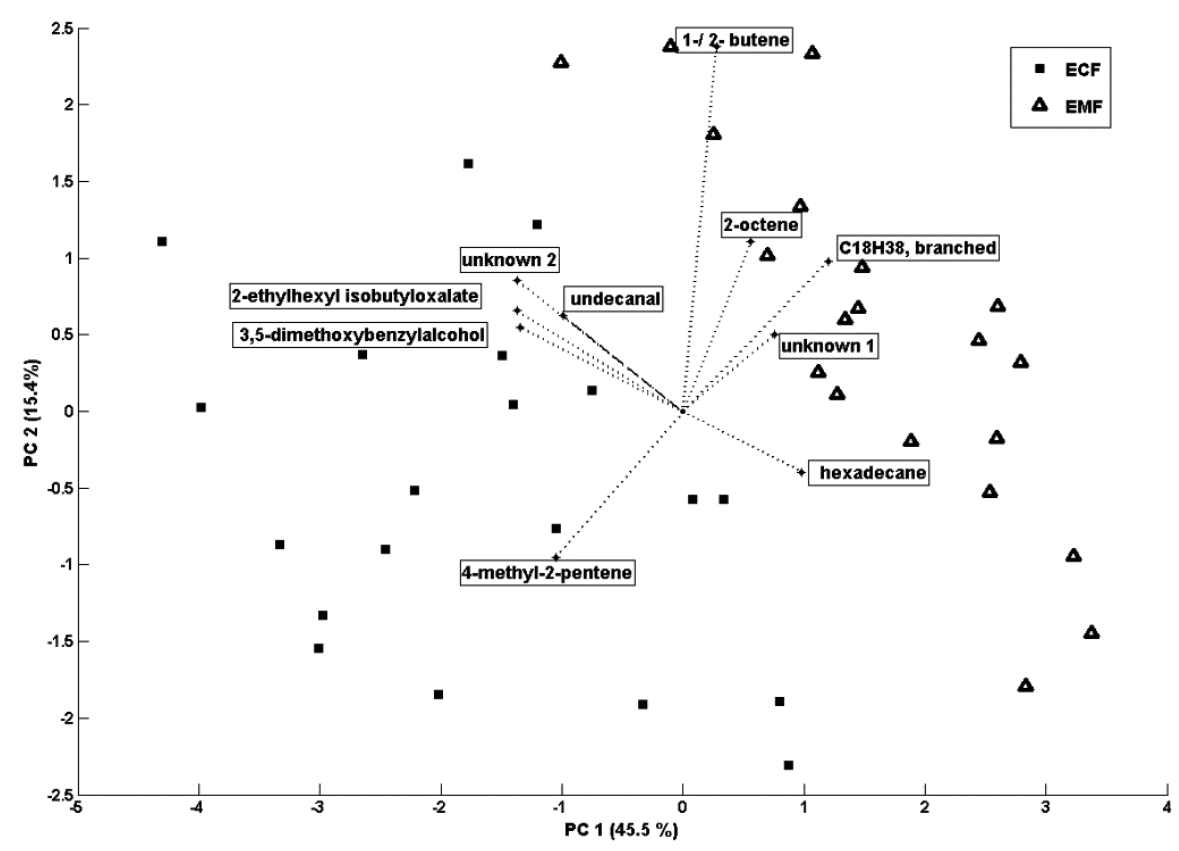

Figure 3. Biplot of PCA analysis performed on the 10 most significantly changed VOCs selected by PLS$D A$ sMC as being different in headspace of ECF (exposed Caco-2 Flasks). Each square and triangle correspond to a single sample (chromatogram) and each line corresponds to a compound.

\section{Discussion}

The aim of this study was twofold: introduction and validation of a newly established system for collection of headspace air from cell cultures and implementation of an experimental design to show a potential application of the system. We have demonstrated that combination of $1 \mathrm{~h}$ collection in the system, GC-tof-MS measurement of the samples and multivariate data analysis, allows detection of VOCs released in each sample set up, as well as spotting subtle, but discriminative differences between them. At the same time, we demonstrated good system reproducibility.

Since the system was designed to mimic conditions normally present in culturing stoves, it was expected to be similarly favourable for cell growth. A post-collection 
mean cell viability of $93.1 \%$ indicated that no substantial cell death occurred during sampling, confirming our expectations.

Several 'bioreactor-like' systems with long term incubation times (12h-24h) were introduced previously to study volatiles being either released or consumed by cancer cell lines (e.g., HepG2, A549, NCl-H446, SK-MES-1) in comparison to medium controls or normal cells (e.g., BEAS-2B, HBEpC, hFB) [10, 23-27]. Such an approach is thought to increase the concentration of released compounds making their detection easier. Determination of cancer-specific VOCs could potentially enable an early, possibly even preventive cancer diagnosis. However, these systems for cells headspace collection require a long-term incubation time. The accumulation of products formed during the incubation time also changes the culturing conditions and may adversely influence cell viability and results, whereas ideally the system should prove to be favorable for cell growth, as well as reproducible in collection output. Long term incubation also limits the possible application of the systems invented so far, as studding variety of exposures effect on VOCs composition will often require fast collection, capturing the change triggered by studied in vivo agent.

Because short collection time (in our case $1 \mathrm{~h}$ ) was found to be sufficient to detect considerable amounts of VOCs present in headspace air, we not only got an insight into VOCs released/consumed by cell lines, but also have the possibility to alter cell environment by including different experimental designs to study their impact on VOCs dynamics. In this study, we present an example of such an application: induction of oxidative stress by the exposure of Caco-2 cells to hydrogen peroxide and its effect on VOCs dynamics detected in the system. Hydrogen peroxide is a stimulus known to dose-dependently induce ROS production, decreased antioxidant defences and increased oxidative DNA damage in Caco-2 cells [14, 28, 29]. Oxidative stress related diseases are among the major cited origins of changed VOC-profiles. Increased reactive oxygen species (ROS) production and oxidation of lipids, proteins and other molecules is commonly observed in diseased people. Lipid peroxidation results in volatile hydrocarbon products, e.g., alkanes and alkenes, that can be easily detected in the breath and be a part of the set of compounds that 
discriminate healthy people from diseased ones. Yet, clinical application of breath biomarkers will need confirmation of the discriminative sets toward certain conditions. So far studies are inconclusive on what are the trends in formation of oxidation products. In this study we looked into the effects of oxidative stress on isolated cells under controlled conditions. Volatiles found in analysis 3 (comparison of EMF vs. ECF), included some compounds solely related to medium oxidation and normal cell metabolism, which needed to be excluded. The remaining 10 out of 16 compounds could be considered as being related exclusively to VOCs generation due to oxidation stress imposed on Caco-2 cells.

Tentative identification of the compounds determined in analysis no. 1 was successful for only 8 out of 15 of compounds that came up as significant. Spiking and using chemical standards in future analysis may allow for identification of more compounds and thus give a better and bigger picture of the biology behind its formation. At this time, we have found phenol, 1-methoxy-2-propanone and ethyl benzene to be released by medium and entirely consumed/ metabolised when Caco2 cells were present. Two $\mathrm{C}_{11} \mathrm{H}_{22}$ isomers and heptane were detected solely in headspace of Caco-2 cultures, pointing towards their cell derived origin. 1-/2nonene and pentadecenal were found in the headspace of both medium only and Caco- 2 cultures. Their relative abundances suggest that they were produced by medium and partially consumed/metabolized by Caco- 2 cells.

The study design allowed distinction of compounds related to the oxidative stress imposed on the Caco-2 cells from compounds that showed to be related to medium oxidation (analysis 2) and normal cell metabolism (analysis 1). Ten out of the 16 compounds that were significantly different could be considered as being related exclusively to VOCs generation due to oxidation stress imposed on Caco-2 cells found in analysis no. 3 (comparison of EMF vs. ECF). For analysis no. 3, from 10 significantly changed VOCs connected solely to the influence of oxidative stress on the Caco-2 cells, we were able to tentatively identify 8 compounds. 4-methyl-2pentene was markedly higher in the headspace of the stressed cells, whereas for instance 2-octene and the branched $\mathrm{C}_{18} \mathrm{H}_{38}$ were reduced when comparing exposed cells to exposed medium only. 
Conclusion: the system described here allows for the dynamic sampling of headspace of cell cultures. Sufficient quantities of VOCs formed during cell culture for adequate GC-tof-MS analysis are produced, while cell viability and system reproducibility are high. It enables fast and varied experiments when performed according to an adequate (deductive) study design and could deliver time-resolved production of VOCs.

In the study presented we used a high number of replicate experiments $(n \geq 20)$ which allow for reproducible and representative results. Not all significant compounds could be identified, this might be improved in future studies when chemical standards are available. 


\section{References:}

1. Dallinga, J.W., A. Smolinska, and F.J. van Schooten, Analysis of volatile organic compounds in exhaled breath by gas chromatography-mass spectrometry combined with chemometric analysis. Methods Mol Biol, 2014. 1198: p. 251-63.

2. Smolinska, A., et al., Current breathomics--a review on data pre-processing techniques and machine learning in metabolomics breath analysis. J Breath Res, 2014. 8(2): p. 027105.

3. Dallinga, J.W., et al., Volatile organic compounds in exhaled breath as a diagnostic tool for asthma in children. Clinical and Experimental Allergy, 2010. 40(1): p. 68-76.

4. Pelli, M.A., et al., Breath alkanes determination in ulcerative colitis and Crohn's disease. Dis Colon Rectum, 1999. 42(1): p. 71-6.

5. Bodelier, A.G.L., et al., Volatile Organic Compounds in exhaled air as novel marker for disease activity in Crohn's disease: A metabolomic approach. , in Inflammatory Bowel Disease.

6. Morisco, F., et al., Rapid "breath-print" of liver cirrhosis by proton transfer reaction time-offlight mass spectrometry. A pilot study. PLoS One, 2013. 8(4): p. e59658.

7. Probert, C.S., et al., Volatile organic compounds as diagnostic biomarkers in gastrointestinal and liver diseases. J Gastrointestin Liver Dis, 2009. 18(3): p. 337-43.

8. Verdam, F.J., et al., Non-alcoholic steatohepatitis: a non-invasive diagnosis by analysis of exhaled breath. J Hepatol, 2013. 58(3): p. 543-8.

9. $\quad$ Altomare, D.F., et al., Exhaled volatile organic compounds identify patients with colorectal cancer. Br J Surg, 2013. 100(1): p. 144-50.

10. Wang, Y., et al., The analysis of volatile organic compounds biomarkers for lung cancer in exhaled breath, tissues and cell lines. Cancer Biomark, 2012. 11(4): p. 129-37.

11. Wang, C., et al., Volatile organic metabolites identify patients with breast cancer, cyclomastopathy, and mammary gland fibroma. Sci Rep, 2014. 4: p. 5383.

12. Torres, M.I. and A. Rios, Current view of the immunopathogenesis in inflammatory bowel disease and its implications for therapy. World J Gastroenterol, 2008. 14(13): p. 1972-80.

13. Rezaie, A., R.D. Parker, and M. Abdollahi, Oxidative stress and pathogenesis of inflammatory bowel disease: an epiphenomenon or the cause? Dig Dis Sci, 2007. 52(9): p. 2015-21.

14. Piccolomini, A.F., et al., High hydrostatic pressure pre-treatment of whey proteins enhances whey protein hydrolysate inhibition of oxidative stress and IL-8 secretion in intestinal epithelial cells. Food Nutr Res, 2012. 56.

15. Van Berkel, J.J., et al., Development of accurate classification method based on the analysis of volatile organic compounds from human exhaled air. Journal of chromatography. B, Analytical technologies in the biomedical and life sciences, 2008. 861(1): p. 101-7.

16. Walczak, B., Wavelets in Chemistry. 2000, Amsterdam: Elsevier Science.

17. Eilers, P.H.C. and B.D. Marx, Flexible smoothing with B-splines and penalties. Statistical Science, 1996. 11(2): p. 89-102.

18. Nielsen, N.P.V., J.M. Carstensen, and J. Smedsgaard, Aligning of single and multiple wavelength chromatographic profiles for chemometric data analysis using correlation optimised warping. Journal of Chromatography A, 1998. 805(1-2): p. 17-35.

19. Dieterle, F., et al., Probabilistic quotient normalization as robust method to account for dilution of complex biological mixtures. Application in H-1 NMR metabonomics. Analytical Chemistry, 2006. 78(13): p. 4281-4290.

20. Smolinska, A., et al., NMR and pattern recognition methods in metabolomics: from data acquisition to biomarker discovery: a review. Anal Chim Acta, 2012. 750: p. 82-97.

21. Tran, T.N., et al., Interpretation of variable importance in Partial Least Squares with Significance Multivariate Correlation (sMC). Chemometrics and Intelligent Laboratory Systems, 2014. 138: p. 153-160.

22. Baranska, A., et al., Profile of volatile organic compounds in exhaled breath changes as a result of gluten-free diet. J Breath Res, 2013. 7(3): p. 037104.

23. Filipiak, W., et al., TD-GC-MS analysis of volatile metabolites of human lung cancer and normal cells in vitro. Cancer Epidemiol Biomarkers Prev, 2010. 19(1): p. 182-95.

24. Filipiak, W., et al., Release of volatile organic compounds (VOCs) from the lung cancer cell line CALU-1 in vitro. Cancer Cell Int, 2008. 8: p. 17.

25. Mochalski, P., et al., Release and uptake of volatile organic compounds by human hepatocellular carcinoma cells (HepG2) in vitro. Cancer Cell Int, 2013. 13(1): p. 72. 
26. Sponring, A., et al., Analysis of volatile organic compounds (VOCs) in the headspace of NCIH1666 lung cancer cells. Cancer Biomark, 2010. 7(3): p. 153-61.

27. Sponring, A., et al., Release of volatile organic compounds from the lung cancer cell line NClH2087 in vitro. Anticancer Res, 2009. 29(1): p. 419-26.

28. Calatayud, M., et al., Active films based on cocoa extract with antioxidant, antimicrobial and biological applications. Food Chem, 2013. 139(1-4): p. 51-8.

29. Aherne, S.A., J.P. Kerry, and N.M. O'Brien, Effects of plant extracts on antioxidant status and oxidant-induced stress in Caco-2 cells. Br J Nutr, 2007. 97(2): p. 321-8 


\section{Summary and general discussion}

This thesis is focused on the untargeted analysis of volatile organic compounds (VOCs) in the context of gastrointestinal health and disease. Breath analysis, with its non-invasive nature, is an attractive prospect for diseases diagnosis and monitoring. It is widely assumed that VOCs in breath provide a reflection of a metabolic state of the organism but understanding and capturing that reflection with pinpointing its source is still an incredibly challenging task. In addition, results published in the literature are based on small patient numbers that impacts statistical validity of the results and where it is virtually impossible to study confounding factors [1]. Chapter 2 of this thesis is looking at the general influence of endogenous and exogenous factors on exhaled breath composition to gain better understanding which potential confounding factors must be accounted for when studying exhaled VOCs. Thanks to support of Top Institute Food and Nutrition, we had an opportunity to study confounding factors in breath samples from the 1417 well-characterised participants from the LifeLine (LL) population cohort in the Netherlands: i.e., age, BMI, smoking, blood cell count, several metabolic parameters, and a group of 16 recorded medications. The results showed that smoking impacted the VOCs profile most significantly.

Age, gender and BMI influence an individual's metabolism. Although they impact breath composition to lesser extent than smoking, they should also be considered in study designs. In our study, no evidence was found that cholesterol or triglyceride levels influenced overall VOCs profile. The immune response is expected to influence VOCs, but surprisingly no significant differences were detected related to the number of various types of white blood cells. This latter may be explained by the lack of statistical power, as in this general population cohort the number of people with increased neutrophil counts was small $(n=11)$. Medications can affect both host and/or microbial metabolism and as such were expected to influence VOCs profiles [2]. Within the available cohort set up we were only able to compare VOCs profiles of women taking chemical contraceptives vs. those not taking them. However, no significant impact on VOCs was noted. For all other medications, because we were 
not able to compare individuals with a certain disease taking medication versus individuals with the same disease not taking the medications, it was impossible to distinguish disease effect from treatment effect on VOCs. It is important to note that medication may affect the breath profile and this information should therefore be considered in breath analysis studies [1]. The effect of external factors, i.e., age, gender, smoking, presence of anxiety and depression was also included in Chapter 5, where the use of VOC as a potential diagnostic biomarker for IBS was investigated. No statistically significant correlations were observed, indicating they were not confounding the separating ability of the VOCs profile for diagnosis of IBS.

Chapter 3 and Chapter 4 focus on exploration if and how exhaled breath was affected by dietary changes in healthy individuals. First, a set of 12 volatile compounds distinguished samples obtained during a gluten-free diet from those obtained during a normal diet (Chapter 3). Our findings indicate that a gluten-free diet had a reversible impact on participants' excreted metabolites visible in breath. Nine weeks after ending the gluten-free diet, the VOC profile returned to the one measured prior to intervention. In the research of Palma et al., gluten-free diet appeared to be associated with reduction of polysaccharide intake and since undigested carbohydrates are considered as a main source of energy for commensal microbiota, it might explain the drift of the gut microbial composition [3]. This in turn is expected to be associated with a change of volatile metabolites produced by bacterial communities. Those metabolites when absorbed into the bloodstream, can be further metabolized, and finally be excreted in urine or breath [4, 5]. An interesting observation was that even though the change in VOCs caused by diet followed the same pattern, the dynamic of this change differed between individuals. Heinzmann et.al., in research on stability and robustness of human metabolic phenotypes in response to food challenges, highlights the importance of understanding impact of individual differences in metabolic baseline and in response to dietary interventions. In their study, some individuals displayed greater stability of metabolic profile than others [6]. Therefore, it is possible that differences in dynamic of changes visible in our study could relate to diverse individual metabolic phenotypes. Secondly, in Chapter 4, we investigated whether breath analysis can be indicative for altered 
metabolism of two studied infant formulas, where the only difference was a droplet size. In a double-blind, cross-over design study, 29 healthy, non-smoking adult males, consumed two different milk formulas and delivered exhaled breath samples at various time points. Results showed significant differences in exhaled breath composition between the two formulas 240 minutes after ingestion, which corresponds to the moment of food entering the small intestine. We speculated that this (in part) may be related to differences in the digestion, absorption, or metabolism of nutrients and/or differences in GI motility [7, 8]. Here also a role of microbial activity could be considered, but this was not investigated in that study. No significant changes were observed at earlier time points. Although the exact origin of the discriminating compounds was not confirmed and pathophysiological consequences are not clear, the findings do show the potential of $\mathrm{VOC}$ analyses in fields of nutrition and metabolism.

Furthermore, monitoring the effect of the diet by exhaled breath composition has potential for example to aid checking dietary compliance or to discover different digestion or absorption patterns. Nowadays, there are several simple commercial breath tests available that aid diagnosis of metabolic disturbances. These include for example measurements of carbohydrate malabsorption or detection of small intestine bacterial overgrowth (SIBO). Enzyme activities and organ functions can also be assessed by using stable isotope-labelled probes, such as ${ }^{13} \mathrm{C}$-labelled urea for the diagnosis of the gastric bacterium Helicobacter pylori. An approach based on the use of exogenous VOCs probes (EVOC) as a potential strategy to measure activity of metabolic enzymes in vivo and by that aid development of breath based diagnostic and prognostic tests, was recently proposed by Owlstone Medical. Exogenous volatiles used as a probe allow direct monitoring of the substrate (probe itself) and products of its metabolism. The recent study by Ferrandino et al. has successfully demonstrated the use of exhaled breath limonene as a biomarker for liver cirrhosis [9]. Identifying specific conditions and diseases that can be targeted via this strategy opens a door for more targeted breath applications.

It was suggested that any exogenous VOC that is metabolised by the human body can offer opportunity to assess metabolic enzyme or organ function [10]. Identifying 
specific conditions and diseases that can be targeted via this strategy opens a door for more targeted breath application.

The diagnostic and monitoring potential of VOCs analysis has been described in Chapter 5 and 6. First, we determined a discriminating VOCs profile between clinically confirmed IBS patients, obtained from the clinical Maastricht IBS (MIBS) Cohort, and GI healthy controls. Next, we determined how this specific VOC pattern correlated with GI symptoms in the MIBS and in LL DEEP general population cohort (Chapter 5). A set of 16 VOCs correctly predicted $89.4 \%$ of the IBS patients and $73.3 \%$ of the healthy controls $(A \cup C=0.83)$. To our knowledge, this was the first time that a set of VOCs in exhaled air was able to predict the presence of a prevalent functional GI disorder, which could be considered an important first step forward in the design and development of reliable non-invasive biomarkers for IBS. Random Forest analysis was validated twice to ensure the reliability of the classification and correct selection of the set of discriminatory VOCs. One of the big advantages of the study was the relatively high number of well-characterised people included. As the diagnosis of IBS is based on symptoms (as defined by the Rome III criteria), our second aim was to test whether our VOC biomarker set would also correlate with the severity of GI symptoms. Here results showed that the VOC-biomarker set correlated moderately with a set of GI symptoms in the MIBS $(r=0.55, p=0.0003)$. Since functional GI symptoms observed in IBS patients are also rather common in the general population, we then confirmed that the VOC biomarker showed a moderate but significant correlation with a set of Gl symptoms in the general population $(r=0.54, p=0.0004)$ [11]. Correlation with symptoms shows promising potential of VOCs analysis in evaluation of treatments effect in IBS. As lifestyle factors, comorbid diseases and mediation used are associated with IBS, potential confounding factors should be included in the analysis as also found in Chapter 2. Kruskal-Wallis test showed however no influence from possible confounding factors in distinguishing IBS patients from healthy controls. We have speculated that both increase in inflammation and oxidative stress as well as microbiota changes could be highly related to the discriminatory power of the set. Further in-depth research is however still needed to investigate the origin of the discriminatory VOCs, to confirm 
their identity, and to investigate the potential link to the underlying causes of IBS. Furthermore, to move from the reported potential closer to clinical applications, the results should be validated in an external cohort.

In Chapter 6, the diagnostic value of breath VOCs to monitor mucosal inflammation in inflammatory bowel disease (IBD) was investigated. In 2005, Lechner et al.,. [12] already demonstrated the feasibility of exhaled air as a novel diagnostic tool in the differential diagnosis of GI diseases but used a limited number of IBD patients $(n=10)$ and did not identify the significant compounds. At that time, to our knowledge, it was the first large prospective study evaluating the role of VOCs profiles in exhaled air in relation to disease activity of Crohn's Disease (CD) patients [13]. 140 samples originated from an active disease stage, based on fecal calprotectin ( $F C>250 \mu \mathrm{g} / \mathrm{g}$ ) and 135 samples from inactive disease stage (with a clinical $\mathrm{HBI}$ score $<4$ and serum $\mathrm{CRP}<5 \mathrm{mg} / \mathrm{l}$ and $\mathrm{FC}<100 \mu \mathrm{g} / \mathrm{g}$ ). A third group consisted of samples from 110 healthy controls. A set of 10 discriminatory VOCs correctly predicted active CD in $81.5 \%$ and remission in $86.4 \%$ (sensitivity 0.81 , specificity 0.80 , AUC 0.80 ). Among tentatively identified discriminatory compounds, enhanced levels of alkanes, methylated alkanes, aldehydes were found, which may be connected to increased inflammation. Several studies show that oxygen mediated injury through increased free radical production and impaired antioxidant defense systems plays an important role in the pathophysiology of IBD [14, 15]. Hydrocarbons as the end products of lipid peroxidation show low solubility in blood and are quickly excreted into breath after formation and can be used to monitor the degree of oxidative damage [16]. Hydrocarbons and aldehydes were previously reported to be produced also by intestinal microbiota [17]. In recent work by Smolinska et al., a strong correlation between volatiles in breath and the fecal microbiome was shown in CD patients [18]. It was speculated that this may be due to anabolism/catabolism of volatile metabolites by microbes and/or stimulation/inhibition of microbial growth by metabolites. Volatile metabolites such as short chain fatty acids (SCFAs; butyrate, propionate, acetate) and alcohols (ethanol and propanol) are products of bacterial fermentation mainly from non-digestible oligo- and polysaccharide [19, 20]. SFCAs have been shown to have anti-inflammatory and anti-carcinogenic effects [21]. 
Additionally, acetone and isoprene came up as discriminatory compounds when comparing healthy controls versus active $C D$ or $C D$ in remission (Chapter 6). Both isoprene and acetone were present in high abundances in every breath sample, but they were both found to be part of discriminating sets of compounds in multitude of conditions [22-29]. As they are the result of 'common' biochemical processes in the body, it remains the question whether they ever will be specific enough to distinguish one condition from another. Furthermore, these compounds change considerably unrelated to the diseased conditions and are quite variably exhaled: their breath levels were reported to vary due to the inhalation from ambient air, diet, fasting, resting and exercise and even circadian rhythms [23, 30-34]. These uncertainties, among others are reasons why mentioned VOCs despite reported discriminatory powers have not yet found the way to clinical applications.

Inflammation, oxidative stress, metabolic changes caused by normal and pathological processes as well as microbiome perturbations, are all factors simultaneously reported to be involved in a multitude of conditions. What one can observe in breath profiles may collectively be impacted by these factors. However, it is not yet fully understood how specific VOCs or combinations thereof can be related to shared underlying pathophysiological mechanisms rather than the specific disease condition per se. Furthermore, more validation is needed, not only to study the prospective biomarker sets in new external cohorts, but also to study them against other diseases.

While the statistical significance or discriminatory power of exhaled breath is crucial for development of a test, it is also crucial to understand the biological origin of VOCs to study specificity of its production and to aid interpretation of results. In Chapter 7 , we showed the validation of a system that would allow measuring VOCs in headspace of an in vitro cultured cell line. Considering interest in GI disorders, we have chosen the Caco-2 human, epithelial cell line that has been wildly used as a model for among others gut barrier function. The in vitro system allows for the application of different cell lines, as well as different experimental setups including varying exposure times and treatment options while preserving cell viability. High reproducibility of the collection system was confirmed by checking correlations 
$(p \leq 0.0001)$ between replicate samples. When studying the influence of oxidative stress on the VOCs composition, a total of 10 VOC's showed either increased or decreased abundance in the headspace of the cell cultures due to the presence of the $\mathrm{H}_{2} \mathrm{O}_{2}$ stressor. An advantage of the developed system is that the released compounds accumulate and can be detected without affecting cell viability. Further, our study design ensured relatively high number of replicate experiments $(n \geq 20)$ and inclusion of appropriate controls. Studying the relation between certain biochemical pathways and the excretion of VOCs in vitro, has the benefit of an enhanced level of control, which is not as simple in in vivo studies. In vitro, we can for example isolate much easier the matrix effect by looking at the volatilome produced by cells with medium, by medium itself, by medium exposed to a trigger (e.g., $\mathrm{H}_{2} \mathrm{O}_{2}$ ) and by medium exposed with cells. Thereby, we can exactly pinpoint where discriminatory compounds are coming from and avoid drawing wrongful conclusions. In Chapter 7 , we investigated cells exposure to hydrogen peroxide but in similar manner we could study genetically altered metabolic pathways, cells co-cultures (e.g., with macrophages), exposures to other agents - either oxidative stress inducing and/or suppressing anti-inflammatory properties, or VOCs produces/consumed by pathogens. We presented a potential and a concept but to fully benefit from in vitro experiments it is important to implement standards to confirm the identified compounds and to further study potential biological origin of the changes observed. 


\section{Challenges, choices, and other dilemmas in Breath Analysis}

\section{Different analytical and methodological approaches in VOCs analysis}

There are many different approaches to collect, measure and analyse VOCs, both within the field of breath and headspace analysis. Before designing any breath analysis study several decisions must be made:

- Which fraction of the breath should be collected (e.g., upper airways air, late expiratory air, alveolar air and mixed air)?

- What is the volume needed (e.g., 2/3 of the total collect, the last $150 \mathrm{ml}, 500$ $\mathrm{ml})$ ?

- How to collect the breath (e.g., Bags, Syringes, Bio-VOC sampler, ReCIVA)?

- What VOCs are we expecting to capture (e.g., entire spectrum for untargeted analysis, specific subset of volatiles)? What is the spectrum of volatility and polarity?

- What types of sorbent to use to trap the volatile compounds (e.g., Tenax TA\&GR, Carbograph, Carboxen)?

- Under what conditions should the adsorption tubes be stored before chemical analysis and for how long?

- What analytical equipment and method parameters would allow detection of these compounds (e.g., GC-MS, IMS, PTR-MS, SIFT-MS)?

- How to control instrumental variation and introduce Quality Controls?

- How to perform data pre-processing?

- What machine learning tools are most appropriate for the posed question?

- How to obtain identification of the unknown compounds?

First, more information will be given in relation to breath collections dilemmas, choices around breath preconcentrating, breath measurement and analysis with some advantages and disadvantages discussed. Then, the subject of standardisation will be discussed and its meaning to VOCs research. 


\section{Breath collection}

Several air compartments of the lungs can be collected, i.e., upper airways air (commonly known as dead space air), late expiratory air, alveolar air and mixed air. This is visualized on Figure 1. Collection of dead space air, accounts for around 150 $\mathrm{ml}$ of air and has a high resemblance to inhaled air and therefore is least informative about endogenously produced volatiles [35]. Collection of late expiratory air is defined in the least detail and there is no standard practice in place [36]. Due to that, different volumes of initial breath are discarded by different research groups, varying from the first few seconds of exhalation [37, 38], removal of one-third [39], half of each sample [40], or use of specific final volume (such as 100 [41] or $150 \mathrm{ml}$ [42]). The actual volume of dead space can be calculated based on the tidal volume of an individual and will vary due to bronchoconstriction, as well as different breathing patterns $[43,44]$. Simply discarding one part of a breath volume, for all individuals, based on averaged out theoretical calculations is likely to bring an error. Alveolar air collection [45-47], is biologically, the most relevant one as this relates to partition, where gas exchange takes place. It will contain the most relevant VOCs, originating from the body via systemic circulation. For a reliable alveolar air collection, simultaneous monitoring with capnography is highly recommended [48]. End-tidal $\mathrm{CO}_{2}$ concentrations fluctuate during the different phases of breathing [36]. Relatively low levels of $\mathrm{CO}_{2}$ are present in Phase I, representing dead space air, which rise during the transitional Phase II (between dead space air and alveolar air) and finally reach a plateau, signaling the start of alveolar Phase III. With a $\mathrm{CO}_{2}$ sensor, air of Phase III can reliably be collected. Last, but not least, is the collection of total mixed air [11, 49], consisting of both dead space air and alveolar air. The one obvious advantage of that method is its simplicity. Less complex approaches often prove to bring less error and as such, especially in large scale studies, are worth considering. What needs to be noted is that mixed air will contain more environmental contamination from dead space air, and from nose and mouth. These need to be considered when planning collection, measuring samples and later in statistical analysis. 


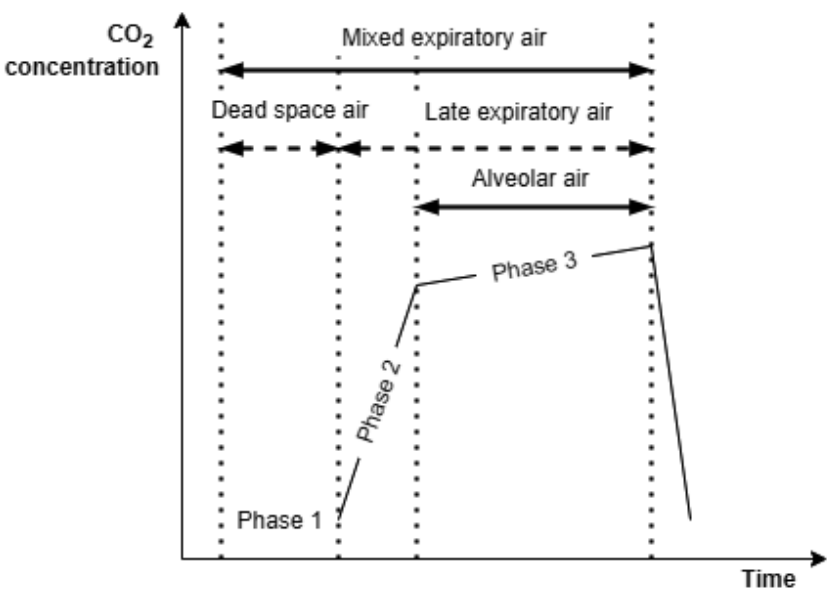

Figure 2. Schematic representation of air compartments and in relation to capnograph, breath phases and air compartments. Modified from Miekisch et.al.,2008 [50]

\section{Sampling Devices}

There are many breath collection containers and devices available in the scientific community. Most often used are polymer bags including Tedlar [51-53] and Mylar bags [54]. Marks International has released Bio-VOC sampler [42, 55] and Menssana Research released breath collection apparatus BCA [56] both for a late expiratory air collection. Some research teams use glad vials and gas-tight syringes [57-59]. The ideal collection container should be durable, cost and user friendly, chemically inert, and non-penetrable for environmental VOC contaminations nor for leakage of breath VOCs. In our research group, we have adapted the mixed breath collection by use of 3-5 $\mathrm{L}$ Tedlar bags for adult study participants and $1 \mathrm{~L}$ bags for children. Tedlar bags are made from polyvinyl fluoride (PVF), being inert to a wide range of compounds and having a relatively good resistance to gas permeation. In previous studies, we have shown that the contribution of dead space air does not impact the sensitivity of measuring VOCs by gas chromatography time-of-flight mass spectrometry (GC-tof-MS) [35, 53]. This approach was done with the assumption that even though dead space air may introduce contamination including environmental background compounds, as well as originating from mouth and nose, 
their distribution will be spread among the study population and as such it should not introduce significant differences to studied groups.

The ReCIVA Breath Sampler available from Owlstone Medical, was developed in collaboration with multidisciplinary leaders in the breath research that joined forces to tackle robustness and reliability issues around breath sampling devices. Today ReCIVA is used in over hundred academic and clinical research sites worldwide and is a great choice for reliable and user-friendly collection. Due to the incorporation of pressure and $\mathrm{CO}_{2}$ monitoring, it allows collection of VOCs from the exact compartments, including alveolar air. The supply of clean air additionally reduces the exogenous background in a sample. With this type of collection, the exhaled VOCs are directly transferred to the thermal desorption tube. With other methods that transfer is the subsequent, manually performed step in the sampling procedure. Prolonged periods between collection in the bag or other container and preconcentration of VOCs onto the tube, may lead to some loss of VOCs. At present, ReCIVA is the collection method of choice and is being used in several breath studies carried out by also Maastricht research team.

\section{Breath preconcentration}

After collection, pre-concentration of the VOC sample is needed to detect VOCs present in very low concentrations, which is the case for majority of studies. This can be done with the use of thermal desorption (TD) tubes, a needle trap device (NTDs), or solid phase micro extraction (SPME). In their review, Lawal et al. showed that TD tubes are used in nearly half of all the studies published [36]. While Carboxen packed tubes are suitable for trapping highly volatile organic compounds $\left(\sim \mathrm{C}_{2}-\mathrm{C}_{4}\right)$, Tenax sorbents might be a better choice to trap the less volatile VOCs in breath $\left(\sim \mathrm{C}_{7}-\mathrm{C}_{15}\right)$ [60]. Volatility of the compounds collected, their polarity, sample volume and the humidity should all be considered when the choice is being made. Nowadays, one can compare and choose the absorbent or construct a multi-bed tube for a specific range of analytes across various sample volumes. In the procedure developed by our research team, after collection of breath in the Tedlar Bag, stainless-steel twobed sorption tubes, filled with carbograph 1TD/Carbo-pack X (Markes International, 
Llantrisant, UK) was connected with one end to the bag itself and with another end to the vacuum pump. The content of the bag is 'pulled' inside the tube. The adsorption tube can then be measured or stored for further analysis. At the time the research in this thesis was performed, samples were stored at the room temperature. Since then, a study on the stability of 74 exhaled breath compounds suggested that analyzing samples by day 14 would minimalize a potential 1-2 standard deviations gain or loss in VOC concentrations [61]. When comparing three storing temperatures $\left(4^{\circ} \mathrm{C}, 21^{\circ} \mathrm{C}\right.$ and $\left.37^{\circ} \mathrm{C}\right), 4^{\circ} \mathrm{C}$ was found to be optimal for compound stability [61]. Kang et al. have examined the stability of compounds stored on dual bed Tenax TA: Carbograph during 12,5 months at $-80^{\circ} \mathrm{C}$. Results showed that maximum storage duration under these conditions is 1.5 month with $94 \%$ of the VOCs being stable [62]. More research including a wider range of compound evaluated should be performed to confirm the preferable conditions for storage of thermal desorption tubes. It is also important to understand optimum purging requirement to remove water prior to storage and if/how this is affected by different storing temperatures.

\section{Breath Measurements}

GC-MS [53, 63, 64] is currently considered a gold standard technique for determination of VOCs in breath, allowing detection of a wide range of compounds, complete profile recognition and single VOC identification. Identified VOCs are easier to compare across the studies and can be related to underlying disease processes. Detailed parameters and description of our approach is mentioned throughout the chapters of this thesis and have been described more extensively by Van Berkel et al., [53]. GC-MS delivers high level of reproducibility [63, 65] and highly accurate chromatograms and it is especially valuable for analysis where the aim is to discover, identify and quantify compounds of interest. It plays a vital and critical role in providing evidence needed to support clinical relevance of potential biomarkers before those can be considered for direct, online testing in the clinic. For that, GC-MS devices are no longer suitable. Proton Transfer Reaction Mass Spectrometry (PTR-MS) [66], and Selected-Ion Flow Tube Mass Spectrometry (SIFT-MS) [67] can be used for real time measurements, and monitoring changes occurring rapidly, such as the influence of exercise, heart rate or ventilation [35]. In 
the work of Amann et. al., PTR-MS was described as a powerful alternative for online monitoring of biochemical reactions in the body [68]. Inability to provide chemical identification of the compounds is its biggest disadvantage. That is not a case for SIFT-MS [67] that even though also based on chemical ionization, allows identification and quantification of VOCs.

\section{Handling Omic- Data}

One of the challenges of breathomics, similarly to other omics, is the ability to analyze the tremendous amount of data. Breath samples contains thousands of volatiles, including the ones of interest coming from endogenous sources and connected to host metabolism, as well as exogenous ones, originating from the inhaled air and its contaminants. When it comes to statistical analysis, the goal is to choose the right tools that will allow to extract the information of interest and neglect the irrelevant one. Various machine learning methods are available nowadays to identify relevant and/or discriminating VOCs [69].

Before data can be analyzed, a preprocessing pipeline needs to be established to deal with spectral noise and differences in baseline [70]. Denoising, baseline correction, alignment across all samples, peak picking, automated peak matching, as well as converting peak areas into a data matrix are all steps to be taken to receive a reliable base for further analysis. This was described in detail by Smolinska et.al., [69].

A major aim of machine learning in breathomics is to explore and understand data complexity. Several characteristics need to be considered. Within breath data set, the number of detected compounds can reach thousands and in general, will by far exceed the number of subjects included. For further analysis, the number of variables needs to be reduced by removing redundant, repetitive, and irrelevant information. Before the actual variable selection strategy can be applied, compounds that are present in small quantity of the studied samples should be removed. In our case, compounds that were present in less than $20 \%$ of samples in at least one of the studied groups were eliminated as they do not carry representative value for the studied objectives. 
As a subsequent step, an exploratory analysis by means of unsupervised approaches is a good way to gain an unbiased view of the data. Most frequently used is Principal Component Analysis (PCA) that allow visualization and distribution of samples, certain outlier detection, as well as spotting eventual grouping of data points and overall trends in the data. It extracts and displays the systematic variation in the data, provides a summary of all samples and shows how they are related to each other. The relation among measured VOCs impacting separation between samples can also be visualized. More detailed explanation of PCA and its interpretation can be found in Chapter 3.

The next step of analysis is to use a priori knowledge, e.g., patient labels, treatment groups and other subject characteristics in supervised analyses. This approach enables finding discriminatory compounds and building predictive models. Although, supervised analysis is powerful, improperly used can lead to biased results. To avoid overfitting, predicted algorithms need to be validated by means of different types of cross validation within the studied set, by use of internal validation set, bootstrapping mentioned later, or ideally in a new independent data set.

Supervised technics can be split into linear and non-linear analyses. Linear discriminant analysis (LDA) is a fast and powerful technique regularly applied in breath research. However, for it to be reliable few steps are required to counteract its limitations. Since LDA assumes normal distribution of the data and since it can only be applied if the number of samples is larger than number of measured compounds - breath data requires transformation to a normal distribution and substantial variable reduction. Example of linear method suited for breath analysis, is Partial Least Square (PLS) and its classification version- Discriminant Analysis (PLS-DA), which can be performed to find VOCs that discriminate between studied groups. PLS-DA resembles PCA and is a latent variable method [71]. While the first principal component (PC) in PCA is constructed in the direction of the highest variance in the data set, the latent variable (LV) of PLS is built in the direction that explains the highest covariance between VOC data and examined class, leading to increased separation between classes. More about that approach can be found in Chapter 6 [72]. The method was successfully implemented in discriminating healthy 
children from children with allergic asthma, as well as, in recognition of lung cancer $[73,74]$. PLS-DA is a powerful technique; however, it only allows looking at linear relationships in the data, is prone to overfitting and can be affected by outliers.

In complex biological systems, nonlinear relations between compounds are often present. For example, disease subgroups, variations in disease stages, medication use, can additionally alter metabolic profiles and generate relations that cannot be captured by previously mentioned linear tools [75]. Nonlinear statistical tools have more power in terms of prediction but are much more difficult to interpret [69]. Among many nonlinear supervised methods used in pattern recognition Random Forest $(\mathrm{RF})$ is one of the most often applied. In RF, randomly selected subsets of original samples selected with replacement (so called bootstrap aggregating used as training set) and randomly selected sets of compounds are used to build a large collection of uncorrelated trees so called weak classifiers. The samples that were not selected into the training set for classifier building are consequently used to validate the model (so called out-of-bag cases for calculation out-of-bag error). The goal of RF is to build many weak classifiers as different as possible from each other. The number of trees, number of samples in terminal node and the number of random variables being used at each node must be optimized [76]. Random forest was used to select most discriminatory compounds in Chapter $\mathbf{5}$ for IBS recognition and in Chapter $\mathbf{6}$ to find compounds discriminating active $C D$ from subjects in remission and from healthy subjects [13]. It was previously used also for classification of patients with different stages of lung diseases and for COPD and bronchial carcinoma diagnosis [77, 78].

Supervised machine learning allows capturing trends in data and identifying compounds responsible for the differences spotted between studied groups. Nevertheless, we need to prove that these trends are not artificial and can be found also in an independent data set. It is extremely important to validate obtained results [79]. It is recommended to have a large enough sample size to split the data from the start in a training set, which will be used to learn, optimize algorithm, and spot the patterns in data, and a validation set, which is solely used for checking reliability of the findings. Such a validation is applied to both studies of diagnostic nature described in Chapter $\mathbf{4}$ and Chapter $\mathbf{5}$. Ideally one would also validate results in an 
external cohort, recruited at different time points and preferably at different clinical sites.

Despite the major progress in breath research, none of the candidate biomarker sets reported in untargeted studies had reached the clinic yet. Several reasons can be put forward for this and although hard to pinpoint exactly, lack of consistency between studies is an important factor. For instance, for disease as IBD and lung cancer more than 40 VOCs are proposed [80]. A recent review on VOCs in IBS and IBD from breath and faeces samples, also showed little overlap [81]. This heterogeneity in results between studies can (in part) be explained by the varying procedures used for breath sampling and storage, the application of diverse detection platforms, differences in data processing and predictive modelling. Furthermore, most of the biomarker sets are found in single-center studies and are not yet externally validated in multi-center studies, making these biomarkers speculative and as such not equipped to be the base of a diagnostic tool in the clinic.

\section{Standardization in Breath Research}

It is important to consider standardization at every step of the process in each methodological approach and to validate the results in a multicenter study. Attempts are ongoing by the European Respiratory Society to define standard guidelines for sampling procedures [82]. Furthermore, within the International Association of Breath Research (IABR) [83] tasks forces are working on harmonizing breath sampling and analysis techniques including QC standardization between labs.

While some standardized practices could be implemented across different methodological platforms (e.g., minimum reporting requirements on number of samples, number of variables, demographics, including food, smoking, medication use and consistent use of reporting units to name few), other will depend on the method and instrumentation used. Using a standard gas mixture as a reference to test equipment and any preprocessing steps was proposed by one of the priorities for future research in exhaled VOCs according to European Respiratory Society [83]. In recent work of Stavropoulos et al., the importance of batch analysis within and across studies was thoroughly explained [82]. In recent work of Stavropoulos et al., 
the importance of batch analysis within study and cross studies was thoroughly explained [84]. Technical variation in GC-tof-MS data can come from many sources. For example, changes in column performance, can introduce gradual drift in instrumental response and influence retention time, peak shape, sensitivity, and accuracy. One way to deal with batch effect is to use internal standards and QC samples at regular intervals. In metabolomic studies, $Q C$ are typically prepared by pooling samples of blood or urine [85], in breathomics, a QC mixture must be carefully composed so that it resembles composition and concentration ranges of metabolites within a study population. To aid compound identification and quantification, internal standards could be used. Identification of compounds based solely on spectral library matching, which often still is the case, should not be considered as a solution and requires confirmation by pure standards. Attempts were made to list compounds in human volatilome with inclusion of Chemical Abstract Service (CAS) numbers, but this requires continuous updating for more metabolites [86, 87]. By 2014, 210 VOCs were confirmed in human breath with the use of pure standards [88]. The use of internal standards and QCs with known concentrations of compounds does allow establishing linear range of the analytical instrumentation and monitoring analytical variations of the instruments. Reporting on Lower and Upper Limits of Detection (LLOD, ULOD) should also be included when assessing compound quantitatively. It is also of highest importance to standardize both methodology for chemicals injection and preparation of the mixture to ensure a reproducible mix. Furthermore, we should consider aspects such as stability of the mixture in time and regularly inspect quality of prepared standards. Following Good Laboratory Practice (GLP), as well as acknowledging and reporting on analytical error should be standard practice. 


\section{Study limitations}

\section{Differences between Rome III and IV and impact on studies performed}

IBS is a prevalent and burdensome disorders investigated in Chapter $\mathbf{5}$ of this thesis. It is diagnosed based on predominant symptoms as define by the Rome Foundation, which revises the definition every 6-10 years. The studies in this thesis were performed according to the Rome III criteria, published in 2006. Since then, stricter, the Rome IV revision was completed and released in 2016 [89]. Consequently, the prevalence of IBS by Rome IV was found to be lower, up to $6 \%$. However, others found that updating the criteria from Rome III to Rome IV should not have a major implication for diagnosis of IBS [90]. The effect of the new diagnostic criteria on the finding presented in Chapter $\mathbf{5}$ are not clear and should be checked in future studies. Additionally, it would be interesting to compare VOC profiles between subgroups of IBS and to study correlations with for example microbiome and habitual dietary intake patterns as well as other potential underlying mechanism.

\section{Compound identification}

At the time of the current studies, we were not using external standards to confirm the identity of the compounds. Compounds were putatively identified by means of spectrum recognition using the National Institute of Standards and Technology (NIST) library in combination with spectrum interpretation by an experienced MassSpectrometrist and identification based on retention index of compounds. Some of the compounds were not identified and would be difficult to validate in further studies. 


\section{VOCs in GI Health}

Diagnosis of GI Diseases is incredibly challenging as many GI diseases share the same spectrum of symptoms, such as chronic abdominal pain or changes in stool patterns. IBS and particularly IBD in remission present themselves similarly with regards to symptoms reported [91, 92]. To differentiate them from each other and other conditions, such as colon cancer and/or celiac disease, warrants often invasive and costly endoscopic procedures [93]. Additionally, many diseases have a multifactorial origin and overlapping pathophysiological factors. For example, the intestinal microbiota has been reported to play an important role in both IBS and IBD $[94,95]$. While gut microbiota composition of healthy individuals is characterized by high diversity and stability over time, in individuals with $\mathrm{Gl}$ conditions a dysbiosis and reduction in diversity and stability is often observed [94, 95]. Moreover, the microbiota composition is related to stool consistency [96] and external factors such as antibiotics use and diet [81]. Since diet provides substrates for microbial fermentation and dietary habits are often altered in diseases subjects, it is difficult to disentangle cause and effect when it comes to diet and microbial composition in relation to symptom etiology. Growing evidence suggests, however, that both play an important role in GI health and disease. Thus far, many speculations were reported on the interplay between VOCs and the microbiota composition. Microbes can change VOC output by either influencing host metabolism or by producing VOCs themselves. Upon creation, they would enter the bloodstream and be released in lungs. As previously briefly mentioned Smolinska et al. has shown a strong correlation between VOCs and the gut microbiome in CD patients with 18 metabolites significantly correlated with 19 faecal bacterial taxa $(R=0.91 p$-value 3.5*10-4) in active disease and 17 volatile metabolites were correlated with 17 bacterial taxa $\left(R=0.96 p\right.$-value $2.8^{*} 10-4$ in quiescent disease [18]. A recent review on volatilomics in IBS and IBD focused on cross-referencing significant VOCs found in breath and faecal analysis studies [81]. Results showed, that 1,4- cyclohexadiene, was identified in breath by our research team (Chapter 5) and was also increased in faecal sample found by others [97]. This was the only overlapping compound identified among 6 studies looking at discriminatory compounds in IBS versus 
healthy volunteers (HV). When comparing $C D$ to $H V, 10$ compounds were found in more than one study, 6 of which were reported by the same group using the same or overlapping study population [98-101]. Additionally, propan-1-ol was found to be increased in faeces and breath in four studies including CD patients [98-100,102], suggesting it may be a compound worth further investigation. However, it is important to note that propan-1-ol is also a common disinfectant. In the review of Van Malderen et al., most of the compounds that were described in two or more studies connected to both IBS and IBD are found to be endogenously or exogenously present in food or produced by microbiota. Many of these compounds were SCFA produced by anaerobic bacterial fermentation, that are known from anti-inflammatory properties and helping to mediate the effect of microbes on the host immune function [103]. 


\section{Future Perspectives}

As shown in this thesis, VOCs analysis has potential for monitoring dietary interventions, and $\mathrm{GI}$ disease diagnosis and progression. However, before VOCs analysis can aid diagnosis in GI health and disease, multicenter studies with adequate number of samples, should be performed to validate proposed sets of biomarkers externally, against healthy cohorts and other relevant alike conditions to check biomarker specificity. Moreover, relationship to symptoms, microbiota, diet, treatments should be studied further to better understand underlying mechanisms in relation to VOCs production and its relevance in clinical practice.

Studying breath in an untargeted manner picks up holistic changes in breath content, but it is yet unknown whether these changes are specific enough to certain conditions or whether they are more general markers for underlying mechanisms such as inflammation or microbiome perturbations. Understanding what levels are related to normal metabolism and what ranges are connected to pathological state is necessary. The future breath biomarker needs to raise above normal inter- and intra-individual variation, and above variation that is possibly affected by environment, or instrument itself. It is incredibly important that we ensure high quality processes and good laboratory practices as with having a stable, consistent approach, we are more likely to succeed in determining and keeping expected variation. Ultimately it would be beneficial for the Breath Community to agree on a set of common rules that could be implemented despite the method and set requirements of minimum reporting.

Recently proposed by Owlstone Medical, EVOC strategy for utilizing exogenous probes to measure metabolic responses could be promising alternative to approaches implemented so far. Understanding more about compound origin, their specificity and further studies involving in vitro analysis of cells, microorganism and viruses is a direction needing more attention. Potential ability to discriminate between viral and bacteriological infections would be enormous achievement that would help with the antibiotic resistance by providing a tool to assess introducing antibiotic only in necessary situations. Considering recent pandemic of COVID-19 it 
is obvious to mention in future perspective the potential of studying breath as an option to aid accurate diagnosis of this viral infection. Although, viruses are nonliving entities and as such do not inherently have their own metabolism, it has become clear in the last decade, that viruses dramatically modify cellular metabolism upon entry into a cell and for that reason they may also affect host VOCs. The COVID-19 pandemic raised interest in exhaled breath condensate (EBC) and associated exhaled breath aerosols (EBA) which are both valuable non-invasive biological media containing a wide range of non-volatile molecules, such as cytokines, chemokines, hydrogen peroxide, ammonia, adenosine, leukotrienes, isoprostanes, nitrogen oxides, peptides, DNA and RNA [104, 105]. The initial concept of using EBA as a potentially useful matrix came from an investigation on what is the probative chemical that allow tracking dogs recognize presence of humans/ animals in e.g., woods long after they are gone, and VOCs would likely disappear [106]. In the pilot study of Jendry et al., reported that trained detection dogs can identify respiratory secretion samples from hospitalized and clinically diseased SARS-CoV-2 infected individuals by discriminating between samples from SARS-CoV-2 infected patients and negative controls with average diagnostic sensitivity of $82.63 \%$ and specificity of $96.35 \%$ [107]. It is important to note that also for EBC collection, one of the biggest issue is lack of standardization and joint multicenter research study is needed to address this further [108].

The field of VOCs research has a long way ahead and the focus should be given to standardization and results validation. 


\section{References}

1. Blanchet, L., et al., Factors that influence the volatile organic compound content in human breath. J Breath Res, 2017. 11(1): p. 016013.

2. Beauchamp, J., Inhaled today, not gone tomorrow: pharmacokinetics and environmental exposure of volatiles in exhaled breath. Journal of Breath Research, 2011. 5(3).

3. De Palma, G., et al., Effects of a gluten-free diet on gut microbiota and immune function in healthy adult human subjects. British Journal of Nutrition, 2009. 102(8): p. 1154-1160.

4. Hamer, H.M., et al., Functional analysis of colonic bacterial metabolism: relevant to health? American journal of physiology. Gastrointestinal and liver physiology, 2012. 302(1): p. G1-9.

5. Jacobs, D.M., et al., Non-digestible food ingredients, colonic microbiota and the impact on gut health and immunity: a role for metabolomics. Current drug metabolism, 2009. 10(1): p. 4154.

6. Heinzmann, S.S., et al., Stability and robustness of human metabolic phenotypes in response to sequential food challenges. J Proteome Res, 2012. 11(2): p. 643-55.

7. Armand, M., et al., Digestion and absorption of 2 fat emulsions with different droplet sizes in the human digestive tract. Am J Clin Nutr, 1999. 70(6): p. 1096-106.

8. Borel, P., et al., Digestion and absorption of tube-feeding emulsions with different droplet sizes and compositions in the rat. JPEN J Parenter Enteral Nutr, 1994. 18(6): p. 534-43.

9. Ferrandino, G., et al., Breath Biopsy Assessment of Liver Disease Using an Exogenous Volatile Organic Compound-Toward Improved Detection of Liver Impairment. Clinical and Translational Gastroenterology, 2020. 11(9).

10. Gaude, E., et al., Targeted breath analysis: exogenous volatile organic compounds (EVOC) as metabolic pathway-specific probes. Journal of Breath Research, 2019. 13(3).

11. Baranska, A., et al., Volatile organic compounds in breath as markers for irritable bowel syndrome: a metabolomic approach. Aliment Pharmacol Ther, 2016. 44(1): p. 45-56.

12. Lechner, A., et al., Headspace screening of fluid obtained from the gut during colonoscopy and breath analysis by proton transfer reaction-mass spectrometry: $A$ novel approach in the diagnosis of gastro-intestinal diseases. International Journal of Mass Spectrometry, 2005. 243(2): p. 151-154.

13. Bodelier, A.G.L., et al., Volatile Organic Compounds in exhaled air as novel marker for disease activity in Crohn's disease: A metabolomic approach. , in Inflammatory Bowel Disease.

14. Torres, M.I. and A. Rios, Current view of the immunopathogenesis in inflammatory bowel disease and its implications for therapy. World J Gastroenterol, 2008. 14(13): p. 1972-80.

15. Rezaie, A., R.D. Parker, and M. Abdollahi, Oxidative stress and pathogenesis of inflammatory bowel disease: an epiphenomenon or the cause? Dig Dis Sci, 2007. 52(9): p. 2015-21.

16. Miekisch, W., J.K. Schubert, and G.F. Noeldge-Schomburg, Diagnostic potential of breath analysis--focus on volatile organic compounds. Clin Chim Acta, 2004. 347(1-2): p. 25-39.

17. Sagar, N.M., et al., The interplay of the gut microbiome, bile acids, and volatile organic compounds. Gastroenterol Res Pract, 2015. 2015: p. 398585.

18. Smolinska, A., et al., Volatile metabolites in breath strongly correlate with gut microbiome in CD patients. Anal Chim Acta, 2018. 1025: p. 1-11.

19. Nicholson, J.K., et al., Host-gut microbiota metabolic interactions. Science, 2012. 336(6086): p. 1262-7.

20. Arora, T. and R. Sharma, Fermentation potential of the gut microbiome: implications for energy homeostasis and weight management. Nutr Rev, 2011. 69(2): p. 99-106.

21. Sivaprakasam, S., P.D. Prasad, and N. Singh, Benefits of short-chain fatty acids and their receptors in inflammation and carcinogenesis. Pharmacology \& Therapeutics, 2016. 164: p. 144-151.

22. Ulanowska, A., et al., The application of statistical methods using VOCs to identify patients with lung cancer. Journal of Breath Research, 2011. 5(4).

23. Kushch, I., et al., Breath isoprene - aspects of normal physiology related to age, gender and cholesterol profile as determined in a proton transfer reaction mass spectrometry study. Clinical Chemistry and Laboratory Medicine, 2008. 46(7): p. 1011-1018.

24. Mashir, A., et al., Effect of the influenza $A(H 1 N 1)$ live attenuated intranasal vaccine on nitric oxide (FENO) and other volatiles in exhaled breath. Journal of Breath Research, 2011. 5(3). 
25. Alkhouri, N., et al., Isoprene in the Exhaled Breath is a Novel Biomarker for Advanced Fibrosis in Patients with Chronic Liver Disease: A Pilot Study. Clinical and Translational Gastroenterology, 2015. 6.

26. McGrath, L.T., R. Patrick, and B. Silke, Breath isoprene in patients with heart failure. European Journal of Heart Failure, 2001. 3(4): p. 423-427.

27. Barker, M., et al., Volatile organic compounds in the exhaled breath of young patients with cystic fibrosis. European Respiratory Journal, 2006. 27(5): p. 929-936.

28. Gordon, S.M., et al., Volatile organic compounds in exhaled air from patients with lung cancer. Clin Chem, 1985. 31(8): p. 1278-82.

29. Smolinska, A., et al., Profiling of Volatile Organic Compounds in Exhaled Breath As a Strategy to Find Early Predictive Signatures of Asthma in Children. Plos One, 2014. 9(4).

30. King, J., et al., Physiological modeling of isoprene dynamics in exhaled breath. Journal of Theoretical Biology, 2010. 267(4): p. 626-637.

31. Ager, C., et al., Effect of inhaled acetone concentrations on exhaled breath acetone concentrations at rest and during exercise. Journal of Breath Research, 2020. 14(2).

32. Spanel, P., K. Dryahina, and D. Smith, $A$ quantitative study of the influence of inhaled compounds on their concentrations in exhaled breath. Journal of Breath Research, 2013. $7(1)$.

33. Ruzsanyi, V. and M.P. Kalapos, Breath acetone as a potential marker in clinical practice. Journal of Breath Research, 2017. 11(2).

34. Cikach, F.S. and R.A. Dweik, Cardiovascular Biomarkers in Exhaled Breath. Progress in Cardiovascular Diseases, 2012. 55(1): p. 34-43.

35. Boots, A.W., et al., The versatile use of exhaled volatile organic compounds in human health and disease. J Breath Res, 2012. 6(2): p. 027108.

36. Lawal, O., et al., Exhaled breath analysis: a review of 'breath-taking' methods for off-line analysis. Metabolomics, 2017. 13(10).

37. Sanchez, J.M. and R.D. Sacks, Development of a multibed sorption trap, comprehensive twodimensional gas chromatography, and time-of-flight mass spectrometry system for the analysis of volatile organic compounds in human breath. Anal Chem, 2006. 78(9): p. 3046-54.

38. Castellanos, M., et al., Breath gas concentrations mirror exposure to sevoflurane and isopropyl alcohol in hospital environments in non-occupational conditions. J Breath Res, 2016. 10(1): p. 016001.

39. Chen, Y., et al., Breath Analysis Based on Surface-Enhanced Raman Scattering Sensors Distinguishes Early and Advanced Gastric Cancer Patients from Healthy Persons. ACS Nano, 2016. 10(9): p. 8169-79.

40. Bigazzi, A.Y., et al., Breath Biomarkers to Measure Uptake of Volatile Organic Compounds by Bicyclists. Environ Sci Technol, 2016. 50(10): p. 5357-63.

41. Kramer, R., et al., A rapid method for breath analysis in cystic fibrosis patients. Eur J Clin Microbiol Infect Dis, 2015. 34(4): p. 745-51.

42. Dadamio, J., et al., Breath biomarkers of liver cirrhosis. J Chromatogr B Analyt Technol Biomed Life Sci, 2012. 905: p. 17-22.

43. Fowler, W.S., Lung function studies; the respiratory dead space. Am J Physiol, 1948. 154(3): p. $405-16$.

44. Burke, T.V., M. Kung, and N.K. Burki, Pulmonary Gas-Exchange during Histamine-Induced Bronchoconstriction in Asthmatic Subjects. Chest, 1989. 96(4): p. 752-756.

45. Filipiak, W., et al., Comparative analyses of volatile organic compounds (VOCs) from patients, tumors and transformed cell lines for the validation of lung cancer-derived breath markers. Journal of Breath Research, 2014. 8(2).

46. Mochalski, P., et al., Blood and breath profiles of volatile organic compounds in patients with end-stage renal disease. Bmc Nephrology, 2014. 15.

47. Mochalski, P., et al., Blood and breath levels of selected volatile organic compounds in healthy volunteers. Analyst, 2013. 138(7): p. 2134-2145.

48. Miekisch, W. and J.K. Schubert, From highly sophisticated analytical techniques to life-saving diagnostics: Technical developments in breath analysis. Trac-Trends in Analytical Chemistry, 2006. 25(7): p. 665-673.

49. Smolinska, A., et al., Comparing patterns of volatile organic compounds exhaled in breath after consumption of two infant formulae with a different lipid structure: a randomized trial. Sci Rep, 2019. 9(1): p. 554.

50. Miekisch, W., et al., Impact of sampling procedures on the results of breath analysis. Journal of Breath Research, 2008. 2(2). 
51. Amal, H., et al., Breath testing as potential colorectal cancer screening tool. Int J Cancer, 2016. 138(1): p. 229-36.

52. Mochalski, P., et al., Blood and breath levels of selected volatile organic compounds in healthy volunteers. Analyst, 2013. 138(7): p. 2134-45.

53. Van Berkel, J.J., et al., Development of accurate classification method based on the analysis of volatile organic compounds from human exhaled air. Journal of chromatography. B, Analytical technologies in the biomedical and life sciences, 2008. 861(1): p. 101-7.

54. Machado, R.F., et al., Detection of lung cancer by sensor array analyses of exhaled breath. Am J Respir Crit Care Med, 2005. 171(11): p. 1286-91.

55. Phillips, C., et al., Short-Term Intra-Subject Variation in Exhaled Volatile Organic Compounds (VOCs) in COPD Patients and Healthy Controls and Its Effect on Disease Classification. Metabolites, 2014. 4(2): p. 300-18.

56. Zeliger, H.I., Y. Pan, and W.J. Rea, Predicting co-morbidities in chemically sensitive individuals from exhaled breath analysis. Interdiscip Toxicol, 2012. 5(3): p. 123-6.

57. Svensson, S., et al., Determination of aldehydes in human breath by on-fibre derivatization, solid-phase microextraction and GC-MS. J Chromatogr B Analyt Technol Biomed Life Sci, 2007. 860(1): p. 86-91.

58. Guo, L., et al., Exhaled breath volatile biomarker analysis for thyroid cancer. Transl Res, 2015. 166(2): p. 188-95.

59. Wang, C., et al., Noninvasive detection of colorectal cancer by analysis of exhaled breath. Anal Bioanal Chem, 2014. 406(19): p. 4757-63.

60. Dettmer, K. and W. Engewald, Adsorbent materials commonly used in air analysis for adsorptive enrichment and thermal desorption of volatile organic compounds. Analytical and Bioanalytical Chemistry, 2002. 373(6): p. 490-500.

61. Harshman, S.W., et al., Storage stability of exhaled breath on Tenax TA. Journal of Breath Research, 2016. 10(4).

62. Kang, S. and C.L.P. Thomas, How long may a breath sample be stored for at-80 degrees C? A study of the stability of volatile organic compounds trapped onto a mixed Tenax:Carbograph trap adsorbent bed from exhaled breath. Journal of Breath Research, 2016. 10(2).

63. Buszewski, B., et al., Human exhaled air analytics: biomarkers of diseases. Biomed Chromatogr, 2007. 21(6): p. 553-66.

64. Mieth, M., et al., Automated Needle Trap Heart-Cut GC/MS and Needle Trap Comprehensive Two-Dimensional GC/TOF-MS for Breath Gas Analysis in the Clinical Environment. Analytical Chemistry, 2010. 82(6): p. 2541-2551.

65. D, W.J.T.a.S.O., Introduction to Mass Spectrometry: Instrumentation, Applications, and Strategies for Data Interpretation. 2007.

66. Schwarz, K., W. Filipiak, and A. Amann, Determining concentration patterns of volatile compounds in exhaled breath by PTR-MS. J Breath Res, 2009. 3(2): p. 027002.

67. Storer, M., et al., Breath testing and personal exposure-SIFT-MS detection of breath acetonitrile for exposure monitoring. Journal of Breath Research, 2015. 9(3).

68. Amann, A., et al., Applications of breath gas analysis in medicine. International Journal of Mass Spectrometry, 2004. 239(2-3): p. 227-233.

69. Smolinska, A., et al., Current breathomics--a review on data pre-processing techniques and machine learning in metabolomics breath analysis. J Breath Res, 2014. 8(2): p. 027105.

70. Georgios Stavropoulos, D.S., Yaser Alkhalifah, Frederik-Jan van Schooten, Agnieszka Smolinska, Chapter 38 - Preprocessing and analysis of volatilome data. Breathborne Biomarkers and the Human Volatilome (Second Edition), 2020: p. 633-647.

71. Smolinska, A., et al., NMR and pattern recognition methods in metabolomics: from data acquisition to biomarker discovery: a review. Anal Chim Acta, 2012. 750: p. 82-97.

72. Baranska, A., et al., Dynamic collection and analysis of volatile organic compounds from the headspace of cell cultures. J Breath Res, 2015. 9(4): p. 047102.

73. Caldeira, M., et al., Profiling allergic asthma volatile metabolic patterns using a headspacesolid phase microextraction/gas chromatography based methodology. Journal of Chromatography A, 2011. 1218(24): p. 3771-3780.

74. Di Natale, C., et al., Lung cancer identification by the analysis of breath by means of an array of non-selective gas sensors. Biosensors \& Bioelectronics, 2003. 18(10): p. 1209-1218.

75. Smolinska, A., et al., Interpretation and Visualization of Non-Linear Data Fusion in Kernel Space: Study on Metabolomic Characterization of Progression of Multiple Sclerosis. Plos One, 2012. 7(6).

76. Breiman, L., Random forests. Machine Learning, 2001. 45(1): p. 5-32. 
77. Hauschild, A.C., J.I. Baumbach, and J. Baumbach, Integrated statistical learning of metabolic ion mobility spectrometry profiles for pulmonary disease identification. Genetics and Molecular Research, 2012. 11(3): p. 2733-2744.

78. Mazzone, P.J., Analysis of volatile organic compounds in the exhaled breath for the diagnosis of lung cancer. Journal of Thoracic Oncology, 2008. 3(7): p. 774-780.

79. Miekisch, W., J. Herbig, and J.K. Schubert, Data interpretation in breath biomarker research: pitfalls and directions. J Breath Res, 2012. 6(3): p. 036007.

80. Marzorati, D., et al., A review of exhaled breath: a key role in lung cancer diagnosis. J Breath Res, 2019. 13(3): p. 034001.

81. Van Malderen, K., et al., Volatomics in Inflammatory Bowel Disease and Irritable Bowel Syndrome: Present and Future. Gastroenterology, 2020. 158(6): p. S884-S884.

82. Horvath, I., et al., A European Respiratory Society technical standard: exhaled biomarkers in lung disease. European Respiratory Journal, 2017. 49(4).

83. Herbig, J. and J. Beauchamp, Towards standardization in the analysis of breath gas volatiles. Journal of Breath Research, 2014. 8(3).

84. Stavropoulos, G., et al., Implementation of quality controls is essential to prevent batch effects in breathomics data and allow for cross-study comparisons. Journal of Breath Research, 2020. 14(2).

85. Sanchez-Illana, A., et al., Evaluation of batch effect elimination using quality control replicates in LC-MS metabolite profiling. Analytica Chimica Acta, 2018. 1019: p. 38-48.

86. de Lacy Costello, B., et al., A review of the volatiles from the healthy human body. J Breath Res, 2014. 8(1): p. 014001.

87. Boots, A.W., et al., Exhaled Molecular Fingerprinting in Diagnosis and Monitoring: Validating Volatile Promises. Trends in Molecular Medicine, 2015. 21(10): p. 633-644.

88. Amann, A., et al., The human volatilome: volatile organic compounds (VOCs) in exhaled breath, skin emanations, urine, feces and saliva. J Breath Res, 2014. 8(3): p. 034001.

89. Hellstrom, P.M. and P. Benno, The Rome IV: Irritable bowel syndrome - A functional disorder. Best Practice \& Research Clinical Gastroenterology, 2019. 40-41.

90. Aziz, I., et al., How the Change in IBS Criteria From Rome III to Rome IV Impacts on Clinical Characteristics and Key Pathophysiological Factors. American Journal of Gastroenterology, 2018. 113(7): p. 1017-1025.

91. Schoepfer, A.M., et al., Discriminating IBD from IBS: Comparison of the test performance of fecal markers, blood leukocytes, CRP, and IBD antibodies. Inflammatory Bowel Diseases, 2008. 14(1): p. 32-39.

92. De Schepper, H.U., et al., Review article: gastrointestinal sensory and motor disturbances in inflammatory bowel disease - clinical relevance and pathophysiological mechanisms. Aliment Pharmacol Ther, 2008. 27(8): p. 621-37.

93. Hungin, A.P.S., et al., The prevalence, patterns and impact of irritable bowel syndrome: an international survey of 40000 subjects. Alimentary Pharmacology \& Therapeutics, 2003. 17(5): p. 643-650.

94. Rajilic-Stojanovic, M., et al., Intestinal Microbiota And Diet in IBS: Causes, Consequences, or Epiphenomena? American Journal of Gastroenterology, 2015. 110(2): p. 278-287.

95. Sundin, J., L. Ohman, and M. Simren, Understanding the Gut Microbiota in Inflammatory and Functional Gastrointestinal Diseases. Psychosomatic Medicine, 2017. 79(8): p. 857-867.

96. Falony, G., et al., Population-level analysis of gut microbiome variation. Science, 2016. 352(6285): p. 560-4.

97. Ahmed, I., et al., An investigation of fecal volatile organic metabolites in irritable bowel syndrome. PloS one, 2013. 8(3): p. e58204.

98. Walton, C., et al., Analysis of Volatile Organic Compounds of Bacterial Origin in Chronic Gastrointestinal Diseases. Inflammatory Bowel Diseases, 2013. 19(10): p. 2069-2078.

99. Walton, C., et al., Enteral feeding reduces metabolic activity of the intestinal microbiome in Crohn's disease: an observational study. European Journal of Clinical Nutrition, 2016. 70(9): p. $1052-1056$.

100. Dryahina, K., et al., Pentane and other volatile organic compounds, including carboxylic acids, in the exhaled breath of patients with Crohn's disease and ulcerative colitis. Journal of Breath Research, 2018. 12(1).

101. Dryahina, K., et al., Quantification of pentane in exhaled breath, a potential biomarker of bowel disease, using selected ion flow tube mass spectrometry. Rapid Communications in Mass Spectrometry, 2013. 27(17): p. 1983-1992. 
102. Ahmed, I., et al., An Investigation of Fecal Volatile Organic Metabolites in Irritable Bowel Syndrome. Plos One, 2013. 8(3).

103. Vinolo, M.A.R., et al., Regulation of Inflammation by Short Chain Fatty Acids. Nutrients, 2011. 3(10): p. 858-876.

104. Brusselmans, L., et al., Breath analysis as a diagnostic and screening tool for malignant pleural mesothelioma: a systematic review. Translational Lung Cancer Research, 2018. 7(5): p. 520-536.

105. Wallace, M.A.G. and J.D. Pleil, Evolution of clinical and environmental health applications of exhaled breath research: Review of methods and instrumentation for gas-phase, condensate, and aerosols. Anal Chim Acta, 2018. 1024: p. 18-38.

106. Pleil, J.D., M.A.G. Wallace, and M.C. Madden, Exhaled breath aerosol (EBA): the simplest non-invasive medium for public health and occupational exposure biomonitoring. J Breath Res, 2018. 12(2): p. 027110.

107. Jendrny, P., et al., Scent dog identification of samples from COVID-19 patients - a pilot study. BMC Infect Dis, 2020. 20(1): p. 536.

108. Bannier, M.A.G.E., et al., Exhaled Breath Condensate in Childhood Asthma: A Review and Current Perspective. Frontiers in Pediatrics, 2019. 7. 
The main aim of this thesis was to describe the potential of volatile organic compounds (VOCs) analysis to gain insight into different aspects of Gastrointestinal (GI) health and disease. Work collated in this thesis was presented during many scientific conferences and documented in scientific publications and with that, had a chance to become a source of inspiration, new insights and lessons learned to other research teams around the world. The work presented in Chapter 5 on Volatile Organic Compounds in breath as marker for Irritable Bowel Syndrome was rewarded with Joop Roels Impact Award 2013 for Breakthrough Character and Impact on Innovation Potential of the Industry. Sharing methodological and analytical approach to studying breath, and headspace air samples with other likeminded scientist enables knowledge share and increases a collective chance for the Breath Community to succeed. Breath analysis, due to its non- invasive nature, has tremendous potential for clinical diagnosis and monitoring, and research presented in this thesis elaborated and confirmed validity of that even further.

Public awareness of GI Health, its importance to our general wellbeing, its relation to diet and microbiome, had grown substantially since the work in this thesis was carried out. That substantial growth is not only visible in scientific literature but also in multitudes of internationally recognised bestsellers published between 2016 and 2021, such as, both funny and informative 'GUT' of Giulia Enders or a nutritional therapy in a book - 'Be Good to Your Gut' by Eve Kalinik, to name couple. This proves how relevant the subject is to the public and how much people's life is being affected by gut discomfort. As a society we have opened to subject that not long ago was still considered taboo and not only that we have started taking gut health in our own hands. This is guided by the need of patients that are often dissatisfied with the help received by the doctors ultimately leading to experimentation with over-thecounter supplementation and/or dietary change all in a hope of symptom release and improved quality of life. This thesis mainly focuses on Irritable Bowel Syndrome (IBS) and Inflammatory Bowel Disease (IBD). IBS is a heterogeneous disorder with a multifactorial, though not completely understood pathophysiology, including alterations in e.g., intestinal motility, visceral perception, intestinal microbiota, barrier function, low-grade immune activation, and psychosocial factors. IBD is a collective name for Crohn's disease (CD) and ulcerative colitis (UC) that are both chronic 
relapsing inflammatory diseases of the gastrointestinal tract. IBD is generally considered to be result of dysregulated mucosal immune response to gut microbes in genetically susceptible individuals. Both IBS and IBD are prevalent and associated with impaired quality of life and relatively high healthcare costs. Bringing tools to aid diagnosis but also monitor effectiveness of different treatments would be of great benefit to society. High prevalence of GI complains and growing interest in GI Health among the general population accelerated further the pace of research and development. Looking at Fortune Business Insight, the global digestive health market size in USD was 37.93 billons in 2019 with the projection of the growth of up to 71.9 billion in 2027. Accordingly, to International Foundation for Gastrointestinal Disorders the cost to society in terms of direct medical expenses and indirectly by the loss of productivity at work may go above 21 billion per year when only assessing situation in US.

The need for better diagnosis, better, more effective treatments, reduction in healthcare cost burden are subjects of interest of many market players looking to invest in R\&D for the development of novel biomarkers and interventional products. With that in mind the work presented in the thesis can be relevant not only to patients, scientists, and healthcare providers, but also potential investors and private companies including pharmaceutical sector, functional food and beverages, vitamins and dietary supplements segments as specified in Digestive Heath Market Share Product types by Fortune Business Insights and visualised in Figure 1.

Today breath analysis is already used in clinical practice to detect for example small intestinal bacterial overgrowth (SIBO) by measuring hydrogen and methane produced by intestinal bacteria, food intolerances caused by lactulose and/or fructose and for the detection of Helicobacter pylori infection. To explore the potential of breath further and broaden range of clinically relevant tests by using non-targeted exploration of new relevant biomarkers, lots need still to be done. 

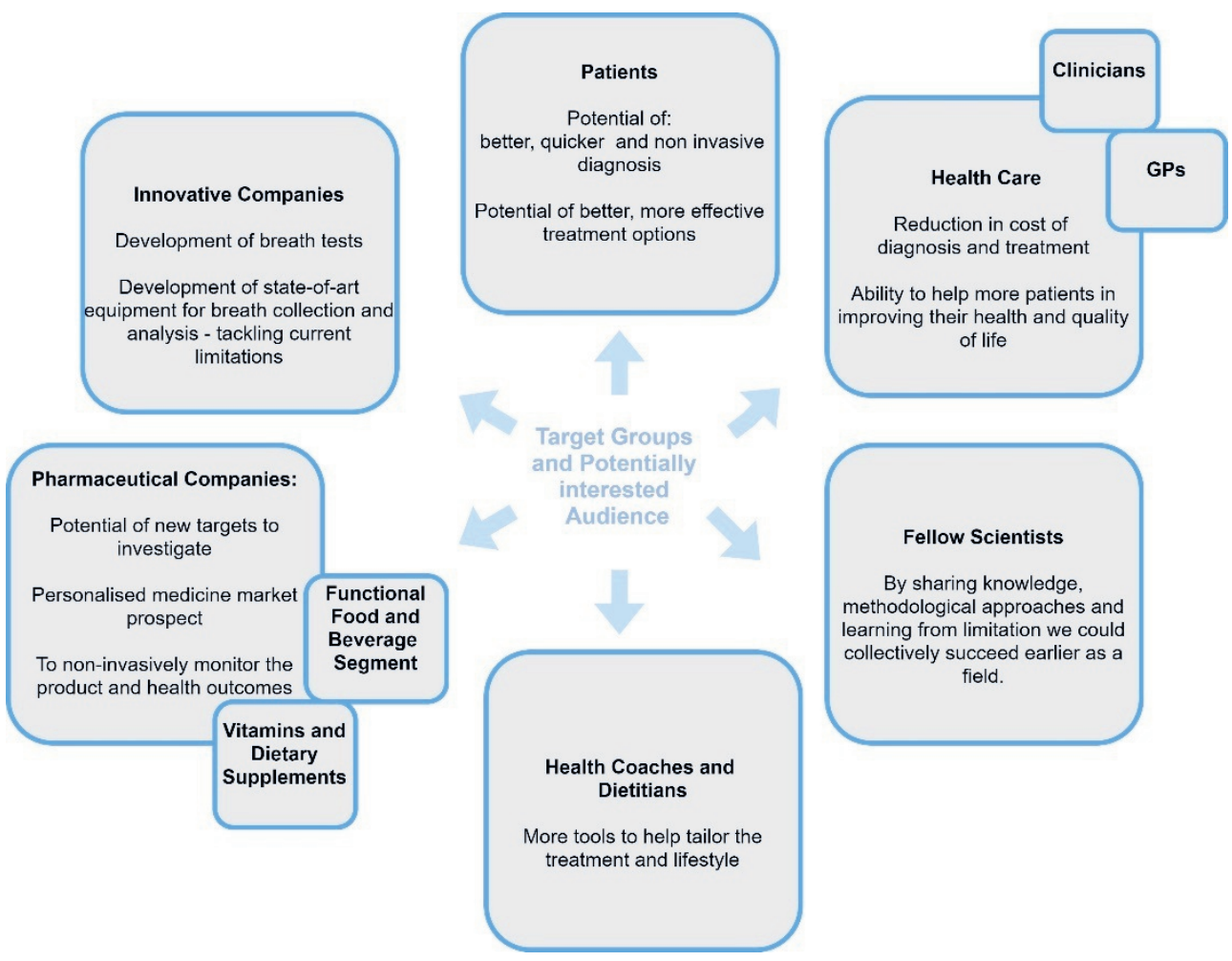

Figure 1. Target groups, areas of influence and potential impact.

One of the problems with using volatilomics as base for diagnosing disease is the heterogeneity in results and the many external factors that can influence the ultimate breath measurement. In Chapter 2 we used a large cohort of 1417 healthy persons to grasp the variations in the human population and to investigate which characteristics influence a single breath measurement the most. Unsurprisingly, results confirmed how significantly smoking affects VOCs in breath and how it is necessary to account for this when designing breath studies. Although age, gender and BMI shown lesser impact on breath composition, they also need to be considered as potential confounding factors. The application of that knowledge was documented also in Chapter 5, where the correlation to cofounding factors was checked to exclude its interference to the separating ability of the VOCs profile for diagnosis of IBS. Both Chapter $\mathbf{3}$ and Chapter $\mathbf{4}$ show impact of dietary changes on breath analyses and although the exact origin of the discriminating compounds was 
not confirmed, the findings do show the potential of VOC analyses in fields of nutrition and metabolism for example to aid monitoring of dietary compliance or to discover different digestion or absorption patterns.

In Chapter 5 a set of 16 VOCs correctly predicted $89.4 \%$ of the IBS patients and $73.3 \%$ of the healthy controls ( $\mathrm{AUC}=0.83$ ). To our knowledge, this was the first time that a set of VOCs in exhaled air was able to predict the presence of a prevalent functional GI disorder, which could be considered an important first step forward in the design and development of reliable non-invasive biomarkers for IBS. Furthermore, in this work we have also investigated the correlation between VOC marker and severity of $\mathrm{Gl}$ symptoms. Moderate but significant correlations was shown to both symptoms in IBS cohort and in general population, showing promising potential of VOCs analysis in evaluation of treatments effect in IBS. Before bringing this further to the clinic we should gain better understanding on the origin of the discriminatory VOCs, confirm their identity, and investigate the potential link to the underlying causes of IBS. Furthermore, to move from the reported potential closer to clinical applications, the results should be validated in an external cohort. This would further allow development of new point of care devices resulting not only in non-invasive diagnosis but also one that is quick, accurate and accessible to clinician.

In Chapter 6, we investigated the diagnostic value of breath VOCs to monitor mucosal inflammation in inflammatory bowel disease (IBD) and concluded that VOCs can be used to discriminate Crohn's disease (CD) patients from healthy controls and between active $C D$ and $C D$ in remission in a real-life cohort. When discriminatory VOCs were combined into single disease activity score, they enabled classifying patients that could not be classified with a combination of faecal and serological markers. Although also here further validation is required, specifically a new multicenter cohort with endoscopic assessment of inflammation as gold standard, the scientific impact of the study is providing feasibility and significant step towards future development of point-of-care devices for assessing disease activity. 
Chapter 7 provides a way to gain insight into what happens with VOCs on cellular level and how can we learn more about VOCs production in vitro to better understand it in vivo. The system for headspace analysis allows the investigation of the influence of certain exposures, treatments, and co-incubations on cell metabolism. The system was validated and the repeatability between subsequent collections was established. The potency of the system was demonstrated by an experiment in which we exposed Caco-2 cells to $\mathrm{H}_{2} \mathrm{O}_{2}$, a well-known oxidative stressor and observed significant changes to 10 VOCs. This work shown promising results and systematic approach to studying VOCs in vitro however, at the time no chemical standards were used for compounds identification and that would substantially increase the impact of the results.

Although the time has passed since the last work in this thesis was published, knowledge shared is as applicable today, as it was at the time. Lack of external validation, proven reproducibility of the results within different cohorts and in between studies, uncertainty with compounds identification and its relation to biological interpretation are still some of the same challenges we are facing today. However, effort of scientific community in bringing in improvement and standardisation is visible, and the field is keep progressing with constantly growing number of new publications. Introduction of more reproducible sample collection e.g., ReCIVA sampler, changes to samples storing conditions, routine application of internal standards are some of the advancements that further increased chances for finding clinically relevant breath biomarkers for disease diagnosis and monitoring.

Work presented is a steppingstone in finding a solution to a current and truly relevant sphere of everyone's health. 
$\because \because$

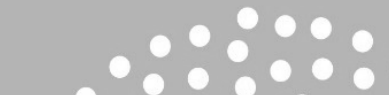

$0_{0}^{0} 0_{0}^{0} 00^{0} 0_{0}^{0}$

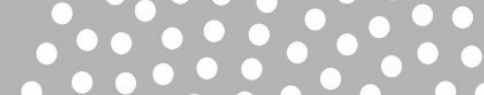

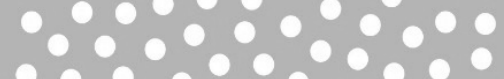

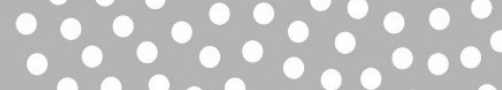

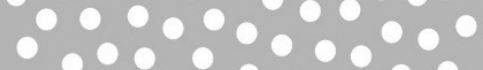

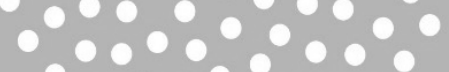

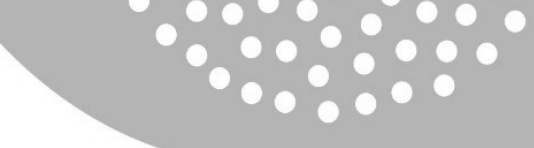

Acknowledgements

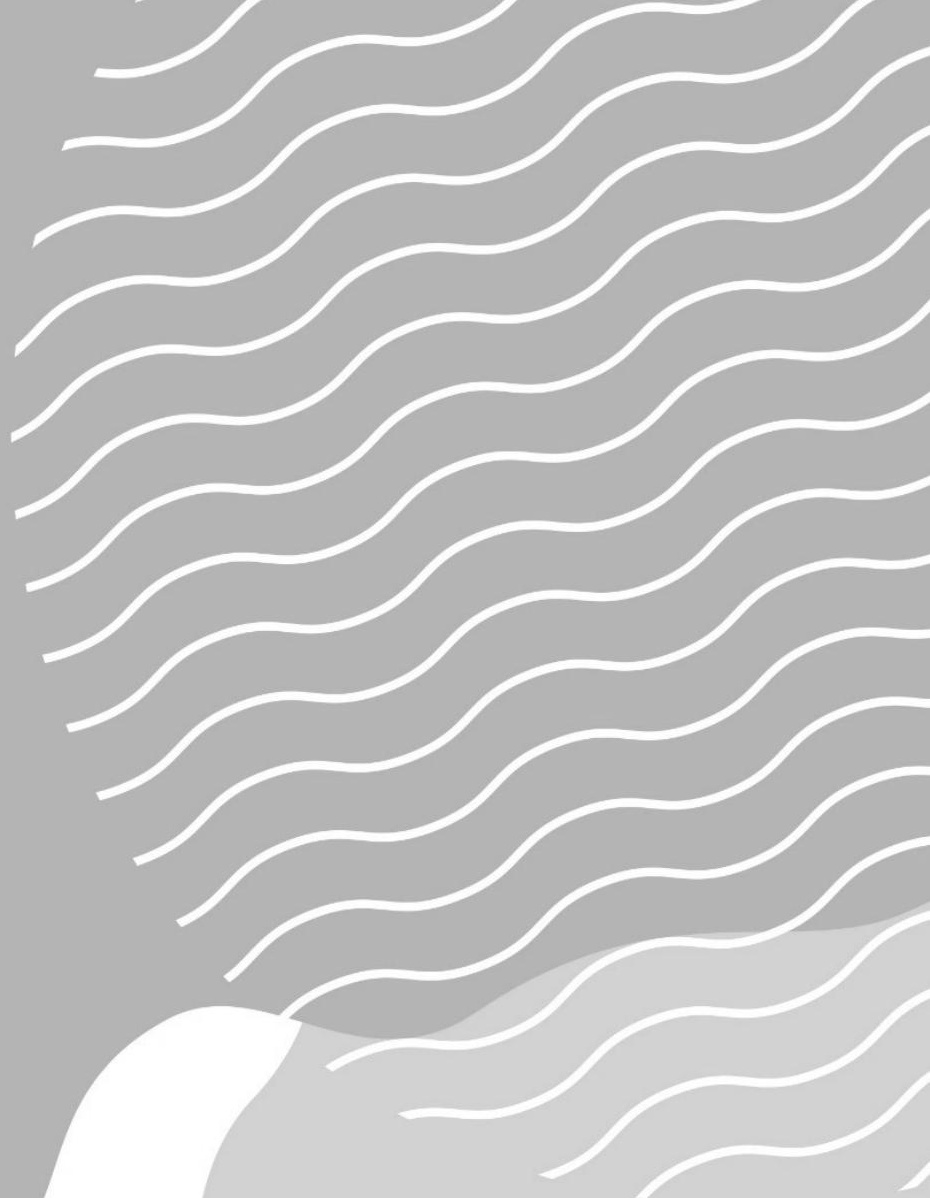


Where do I start? Although it took a while from when I first started my PhD to its completion, I have never lost my passion for breath science and the drive to do my best to help breath research succeed. It gives me great pleasure to finally be able to write this thank you section, as there are so many of you that have directly and/or indirectly added value to this research, thesis, and my life in general. You have all helped me make it happen and I will be forever grateful for that.

I start with my lovely parents without whom I would never be where I am today literally and metaphorically. They not only gave me this beautiful life, instigated strong foundations but also offered the support I needed throughout my life. They have always trusted my choices and although it may have been difficult at times, they found a way to let me go, let me grow and fly, to fulfil my potential. Being a Mum today, I can better understand and appreciate it. I have been away from home, on and off, since I was 17 years old and the ability to do so has had a huge impact on who I am today. It has broadened my horizons, built my appetite for life and love for other cultures. Allow me to say thank you in polish. Kochana Mamo, Tato, dziękuję Wam z całego serca za waszą miłość, wsparcie, troskę w każdej minucie mojego życia. Dziękuję Wam za motywacje tam, gdzie była ona potrzebna, a także za cierpliwość i wiarę we mnie w momentach, gdzie to ona była niezbędna. Dziękuję że pomimo strachu wspieraliście moje wybory. Pozwoliliście mi rozwinąć skrzydła, mieszkać, pracować za granica od młodego wieku, co w dużej mierze ukształtowało mnie jako osobę. Dziś, sama będąc mamą, wiem jak dużo was to kosztowało emocji i troski. Dziękuję wam, że byliście przy mnie w zdrowiu i w chwilach radości a także, w czasie choroby. Byliście i zawsze będziecie moimi pierwszymi 'chirliderami'. Kocham Was z całego serca.

I could not wait any further to thank two extraordinary human beings - my husband, Gwil, and my son, Oliver. You two are the loves of my life, best buddies, best friends, and I refuse to limit 'best' to one. You are my home. I could not be who I am and where I am without the two of you. Gwil, my husband and soulmate, you must be one of the most positive human beings on this planet, not easily phased with much at all. You are the calm oasis for my busy brain. When I met you at Castellmare di 
Stabia in Italy, with the Vesuvius in the background, I could not guess that you will become my husband, but I knew right there and then that I have met an incredibly special someone. It is amazing how well our character traits complement each other and how one can rely on strengths of the other to find comfort or develop further. You helped me so much with finalising this thesis and thank you for doing so. Oli, you are nearly 6 years old as I write this. You are the best son I could have ever asked for and motherhood is the most important 'project' I could ever embark on. You have the kindest nature, and you are a true source of inspiration. Thank you for letting me have the time for completing the work needed for this thesis. It took away more than one holiday day for me to complete it and I promise to make it up in so many family holidays to come. Thank you for keeping me in positive spirits and making me smile every day.

A huge thankyou also to other family members. To my dear brother Krzyś and his wife Ania, for your support throughout the years. It is thanks to you I got my first job in Melrose, Scotland where, besides mastering my table waiting skills, I was able to polish my English and earn money to live a better student life when studying in Poznań. This first summer invitation had started a great adventure for me, one that led to growing confidence, eagerness, and appetite to travel more, ultimately resulting in me pursuing living in Spain, Denmark and, finally, the Netherlands. Thank you also to Jane, who sadly is no longer with us, David, Susan and Carys for making UK feel like home and accepting me into the family.

Thank you to my dear friends whose great support was much needed and appreciated. Gosiaku ty zawsze przy mnie byłaś i wspierałaś bez względu na wszystko, od czasu studiów jesteś dla mnie jak siostra i jestem ci wdzięczna za to że jesteś. Pati, bez względu czy raz w miesiącu, czy raz na rok rozmowa $z$ tobą to zawsze zastrzyk świetnej energii i podróż w czasie do czasów Walencji czy Santorini. Ta pozytywna energia nie raz okazała się pomocna $w$ trakcie tworzenia tej pracy. Dziękuję również Marcie i Madzi -choć dziś wszyscy mieszkamy w innych krajach wiem że zawsze mogę na was liczyć. 
Thank you to all my friends and colleagues from Maastricht. Elcin, Kristine and Carmen you were my rocks throughout my stay in the Netherlands. Thank you for being such good friends. For all the fun times both at university and outside of it. You made Maastricht feel more like home and I will be forever grateful for that. Also thank you to Alie, Danielle, Erik, Misha, Roger, Lou, Danielle P., Leonie, Rianne, Quan, Gesiele for making an amazing atmosphere. Edwin, thank you for all the support with sample handling and running of GC-MS. We had thousands of samples to be measured, stored, analysed and you were a great help in making it happen. Josephina, thank you for all the help with documentation, arranging meetings and this thesis distribution, I really appreciate it.

Huge thank you to Top Institute Food and Nutrition (TIFN) and all my colleagues from GH001 TIFN project. Thank you to Cisca for your great project leadership, Jackie for your positivity and enthusiasm but also Ettje, Gerben, Edith, Sasha, Hauke, Jan, Javier, Ervin, Morris and Thomas for your great teamwork and inspirational chats. A special thank you also to Zlatan, who besides being great colleague to work with, had always made train travelling to and from TIFN meetings a real pleasure.

I would also like to thank all the participants of the studies involved in this thesis: Lifeline Cohort, IBS Maastricht Cohort, CD patients and Healthy Volunteers visiting the outpatient clinic of the Maastricht University Medical Center (MUMC), Volunteers for Gluten free diet study, as well as Strong Study. Without you, none of this would be possible.

Although Owlstone Medical was not involved in the research presented in this thesis or this thesis production, I would like to give a warm thank you, to all my colleagues in Owlstone, who every day give their best, to move the field of breath research further and who together create a wonderful environment to work in. Here I would like to specifically thank Russell. I have to say I am thankful first impressions are not always right! :) You have provided me with exactly right type of management that I needed to develop further. I was able to follow my natural predisposition, discover 
and showcase ways in which I can deliver the most value to the company and ultimately to breath research. Today I know that enabling others is a passion of mine and in my current role I am able to help other scientists fulfill their potential. Today I am able to promote standardisation of practices, collaboration and knowledge sharing as critical areas necessary for further success.

I would like to sincerely thank committee members: Prof. Dr. Edward Dompeling, Prof. Dr. Edwin Mariman, Dr. John Penders, Prof. Dr. Kristin Verbeke and Dr. PierreHugues Stefanuto for taking time assessing my thesis and participation in my thesis defense.

Finally, I would like to thank Prof. Dr. Frederik-Jan van Schooten and Prof. Dr. Jan Dallinga for selecting me for the PhD position and sharing their expertise with me. In doing so, you gave me an amazing opportunity to grow professionally but also exposed me to breath research, a field that I care passionately about. I would like to thank Prof. Dr. Daisy Jonkers and Prof. Dr. Ad Masclee for all the support given throughout and their expertise. Last, but not least, a huge thank you to Dr. Agi Smolinska. Agi - you have not only provided me with support in chemometric and data analysis but also became a friend over the years. Thank you for being there for me!

To Frederik-Jan, Agi and Daisy - my supervisory board, I will forever be grateful to you all, for your patience and not giving up on me defending the fruits of my research. Thank you! 


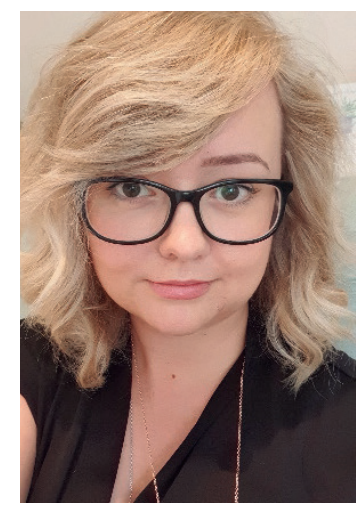

Agnieszka Baranska was born on the 7th of September 1987 in Słupsk, Poland. After finishing secondary school in Słupsk, she started further education in 2006 at Poznan University of Life Sciences, where she studied Biotechnology and graduated with distinction in 2011. Her master thesis: 'Analysis of polymorphic variants of CCND1 gene in patients with differentiated thyroid carcinomas and in Polish population' supervised by Prof. Ryszard Słomski, was carried out in the Institute of Human Genetics in Polish Academy of Science in Poznan. During this study period she has also spent one semester studying abroad at the Technical University of Valencia, Spain. In 2011, she started as a PhD fellow at the Department of Toxicology at Maastricht University, the Netherlands, under supervision of Prof. Dr. F.J. van Schooten, Prof. Dr. D. Jonkers and Dr. A. Smolinska. The research was performed within NUTRIM School of Nutrition and Translational Research in Metabolism at Maastricht University, and part of the research presented in this thesis was funded by the Top Institute Food and Nutrition (TIFN), Wageningen.

In 2016, Agnieszka moved to London, UK with her husband Gwil and newly born son Oliver. After a couple of years she relocated to Cambridge, UK where she is currently working as a Team Lead for Owlstone Medical, global leader in Breath Biopsy for early detection and precision medicine. 


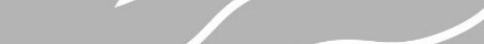

\section{Portfolio}




\section{Publications}

Baranska, A., Smolinska, A., Boots, A.W., Dallinga J.W., F.J. van Van Schooten, F.J.Dynamic collection and analysis of volatile organic compounds from the headspace of cell cultures. Journal of Breath Research (2015)

Baranska, A. ${ }^{*}$, Mujagic, Z. *, Smolinska, A., Dallinga, J.W., Jonkers, D., TigchelaarFeenstra, E.F., Dekens, J.A.M., Zhernakova, A., Ludwig, T., Masclee, A.A.M., Wijmenga, C., Van Schooten, F.J. Volatile organic compounds in breath as marker for irritable bowel syndrome: a metabolomics approach. Alimentary Pharmacology \& Therapeutics (2016)

Baranska, A.*, Tigchelaar-Feenstra E. ${ }^{*}$ Smolinska, A., Dallinga, J.W., Moonen, E.J., Dekens, J.A.M., Wijmenga, C., Zhernakova, A., Van Schooten, F.J. Profile of volatile organic compounds in exhaled breath changes as a result of gluten free diet. Journal of Breath Research (2013)

Smolinska, A., Baranska, A., Dallinga, J.W., Mensink, R.P., Baumgarter, S., Van de Heijning, B.J.M., Van Schooten F.J. Comparing patterns of volatile organic compounds exhaled in breath after consumption of two infant formulae with a different lipid structure: a randomized trial. Scientific Reports (2019)

Bodelier, A.G. ${ }^{*}$ Smolinska, A. ", Baranska, A., Dallinga, J.W., Mujagic, Z., Vanhees, K., van den Heuvel, T., Masclee, A.A., Jonkers, D., Pierik, M.J.*, Van Schooten F.J.* Volatile Organic Compounds in exhaled air as novel marker for disease activity in Crohn's disease: A metabolomic approach. Inflammatory Bowel Disease (2015)

Blanchet, L., Smolinska, A., Baranska, A., Dallinga, J., Wijmenga, C., Van Schooten, F.J. Factors that influence the volatile organic compound content in human breath. Journal of Breath Research (2017)

Mujagic, Z.", Tigchelaar-Feenstra, E.F.. ${ }^{*}$ Zhernakova, A., Ludwig, T., Ramiro-Garcia J., Baranska, A., Swertz M.A., Masclee, A.A., Wijmenga, C., Van Schooten, F.J., Smolinska, A., Jonkers, D.M. A novel biomarker panel for irritable bowel syndrome and the application in general population. Scientific Reports Nature (2016)

Tigchelaar, E.F., Zhernakova, A., Dekens, J.A.M., Hermes, G., Baranska, A., Mujagic, Z., Swertz, M., Muñoz, A.M., Deelen, P., Cenit, M.C., Franke, L., Scholtens, S., Stolk, R.P., Wijmenga, C., Feskens, E.J.M. Cohort profile: LifeLines DEEP, a prospective, general population cohort study in the northern Netherlands: study design and baseline characteristics. BMJ Open (2014)

*Authors share co-first/co-last authorship 


\section{Presentations (poster and oral)}

Baranska, A., Smolinska, A.,Dallinga, J.M.,Mujagic, Z.,Jonkers, D.,Masclee, A.A.M., Wijmenga C. , F.J. van Schooten Volatile Organic Compounds as noninvasive indicator of gut status. $14^{\text {th }}$ Gut Day, Leuven, Belgium (2012)

Baranska, A., Smolinska, A.,Dallinga, J.M.,Mujagic, Z.,Jonkers, D.,Masclee, A.A.M., Wijmenga C. , F.J. van Schooten Biomarkers for GI Health -TIFN- GH001 Project Overview with closer look into metabolomics analysis. NUTRIM Days, Maastricht, The Netherlands (2012)

Baranska, A., Smolinska, A., Dallinga, J., Van Schooten, F.J. Breath samples analysis using GC-TOF-MS. VOCs profiles as biomarkers of health and disease, VLAG PhD week (2012)

Baranska, A., Smolinska, A.,Dallinga, J.M.,Mujagic, Z.,Jonkers, D.,Masclee, A.A.M., Wijmenga C. , F.J. van SchootenGC-MS analysis of volatile organic compounds (VOCs) in exhaled breath from persons suffering from irritable bowel syndrome. $15^{\text {th }}$ Gut Day Conference, Groningen, The Netherlands (2013)

Baranska, A., Smolinska, A., Dallinga, J.W., Moonen, E.J.C., Tigchelaar-Feenstra, E.,Dekens, J.A.M.,Wijmenga, C.,Zhernakova, A.,Van Schooten, F.J. Composition of volatile organic compounds in exhaled breath changes reversibly due to glutenfree dietary intervention $4^{\text {th }}$ TIFN Annual conference, Amsterdam, The Netherlands (2013)

Baranska, A., Smolinska, A., Dallinga, J.M., Mujagic, Z., Jonkers, D., Masclee, A.A.M., F.J. van Schooten Exhaled breath analysis - novel approach towards recognition and characterization of irritable bowel syndrome. 8th International Conference on Breath Research \& Cancer Diagnosis. Toruń, Poland (2014)

Baranska, A., Smolinska, A., Dallinga, J., Van Schooten, F.J. Breath analysis potential applications in dietary interventions. NuGO week, Italy (2014)

Baranska, A., Smolinska, A., Dallinga, J., Van Schooten, F.J. Collection and analysis of volatile organic compounds (VOCs) from headspaces of Caco-2 cell cultures - what, why and how? NUTRIM Days, Maastricht, The Netherlands (2014) 


\section{Other Achievements:}

TIFN team Joop Roels Impact Award 2013 for Breakthrough Character and Impact on Innovation Potential of the Industry for the research on Volatile organic compounds in breath as marker for irritable bowel syndrome: a metabolomics approach.

Nomination for the TIFN Best Publication Award 2016 for the publication: Volatile Organic Compounds in breath as marker for Irritable Bowel Syndrome: a metabolomics approach. Alimentary Pharmacology \& Therapeutics.

\section{Additional Training:}

Bio-Business course, Amsterdam, 2014 WMO-GCP Training/Course by CTCM, Maastricht, 2014

From Epidemiology to Intervention Post graduate course, Italy, 2014 Data analysis of large datasets, 2013

IP workshops for TIFN employees, Wageningen, 2012

Data-mining School, Maastricht, 2012 